MINISTÈRE DE L'ÉDUCATION NATIONALE,

DE L'ENSEIGNEMENT SUPÉRIEUR

ET DE LA RECHERCHE
MINISTĖRE DE LA CULTURE

ET DE LACOMMUNICATION

INSPECTION GÉNÉRALE DES BIBLIOTHĖQUES

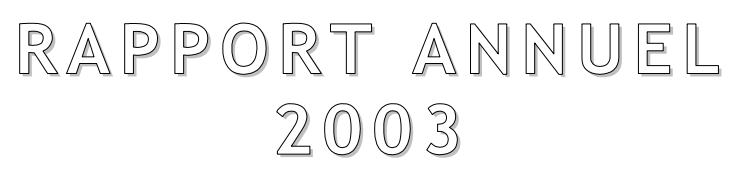

Mai 2004 
INSPECTION GÉNÉRALE DES BIBLIOTHĖQUES 
1.1.1.3. Visites inscrites au programme du ministère de la Culture

1.1.1.4. Visites faites à l'initiative de l'inspecteur

1.1.2. Activités d'expertise et de conseil hors du champ de l'inspection

p. 16

1.2. Activités internationales

p. 17

1.2.1. Activités internationales des membres de l'Inspection

p. 17

1.2.2. Missions en Irak et en Afghanistan

p. 17

1.2.2.1. Évaluation de l'état du patrimoine irakien

p. 17

1.2.2.2. Collections et bibliothèques publiques afghanes

p. 20

1.3. Participation à des instances scientifiques et techniques

p. 22

1.3.1. Conseils, comités et commissions

p. 22

1.3.2. Groupes de travail

p. 23

1.4. Activités administratives

p. 25

1.4.1. Jurys de concours et d'examens

p. 25

1.4.2. Gestion et évaluation des personnels

p. 26

1.4.3. Collaboration avec d'autres instances de conseil, d'évaluation et de contrôle

p. 26

1.5. Autres activités scientifiques et professionnelles

p. 28

2.1.1. Les groupes de travail

p. 31

2.1.2. Les concours

p. 31

2.1.3. Études et programmes

p. 33

2.2. Bilan thématique des travaux

p. 33

2.2.1. Principaux dénominateurs des missions d'inspection

p. 33

2.2.1.1. Bibliothèques publiques

2.2.1.2. Bibliothèques d'enseignement supérieur

p. 33

p. 36

2.2.2. Études concernant des bibliothèques ou des groupes de bibliothèques

p. 41

2.2.2.1. L'audit de la Bibliothèque nationale de France

p. 41

2.2.2.2. Les bibliothèques universitaires d'île-de-France

p. 47

2.2.2.3. La lecture publique en Polynésie française

p. 51

2.2.3. Thèmes transversaux

p. 57

2.2.3.1. Le patrimoine imprimé

p. 57

2.2.3.2. Le harcèlement moral

p. 60 
2.3. Les bibliothèques territoriales et la décentralisation : état des lieux

p. 62

2.3.1. Introduction

p. 62

2.3.1.1. Origine et limites de ce travail

p. 62

2.3.1.2. Le paysage administratif

p. 63

2.3.2. L'application de la décentralisation aux bibliothèques territoriales

p. 66

2.3.2.1. Les bibliothèques municipales

p. 66

2.3.2.2. Les bibliothèques centrales de prêt

p. 70

2.3.3. Le rôle de l'État vu par lui-même

p. 71

2.3.3.1. Les mesures prises

p. 71

2.3.3.2. Les mesures qui n'ont pas été prises

p. 75

2.3.3.3. Une curiosité

p. 76

2.3.4. Quel bilan?

2.3.4.1. Les bibliothèques municipales

p. 77

p. 77

2.3.4.2. Les $B D P$

p. 90

2.3.5. Nouvelles mesures : hypothèses

2.3.5.1. Le concours particulier et les conservateurs d'État

p. 96

2.3.5.2. Une alternative à l'État parisien : l'échelon régional

p. 96

p. 100

2.3.6. Pour aider à la réflexion

p. 103

p. 103

2.3.6.1. A propos du conseil et de l'évaluation

p. 104

2.3.6.2. Le patrimoine

p. 105

2.3.6.3. Les réseaux

p. 107

2.3.7. Conclusion

p. 108

3. LE FONCTIONNEMENT DE L'INSPECTION GÉNÉRALE DES BIBLIOTHĖQUES

p. 111

3.1. Le personnel

p. 111

3.1.1. Les inspecteurs généraux

p. 111

3.1.2. Le secrétariat

p. 112

3.2. Organisation matérielle

p. 112

3.2.1. Locaux

p. 112

3.2.2. Crédits et équipement

p. 112

3.2.2.1. Le ministère de la Culture et de la Communication

p. 113

3.2.2.2. Le ministère de la Jeunesse, de l'Éducation nationale et de la Recherche

p. 113

3.3. Le fonctionnement

p. 113

3.3.1. La réforme du statut

p. 113

3.3.2. Organisation administrative

p. 115

3.3.3. Contrôle

p. 115 
Annexe 1 : Établissements inspectés ou visités en 2003

Annexe 2 : Travaux et publications de l'IGB en 2003

p. 121

Annexe 3 : Revue de presse

p. 123

Annexe 4 : Jurys de concours présidés par les IGB en 2003

Synthèse des rapports

Annexe 5 : Textes concernant l'IGB

État au 31 décembre 2003

p. 131

Annexe 6 : Répartition des zones d'inspection (2004)

p. 137

Annexe 7 : Présidences de jurys de concours et d'examens (2004)

p. 139

Annexe 8 : Informations pratiques concernant l'IGB

p. 141

Le rapport annuel de l'Inspection est également accessible sur le site de l'IGB :

www.education.gouv.fr/syst/igb rubrique "Publications" 


\section{LISTE DES ABRÉVIATIONS ET DES SIGLES UTILISÉS}

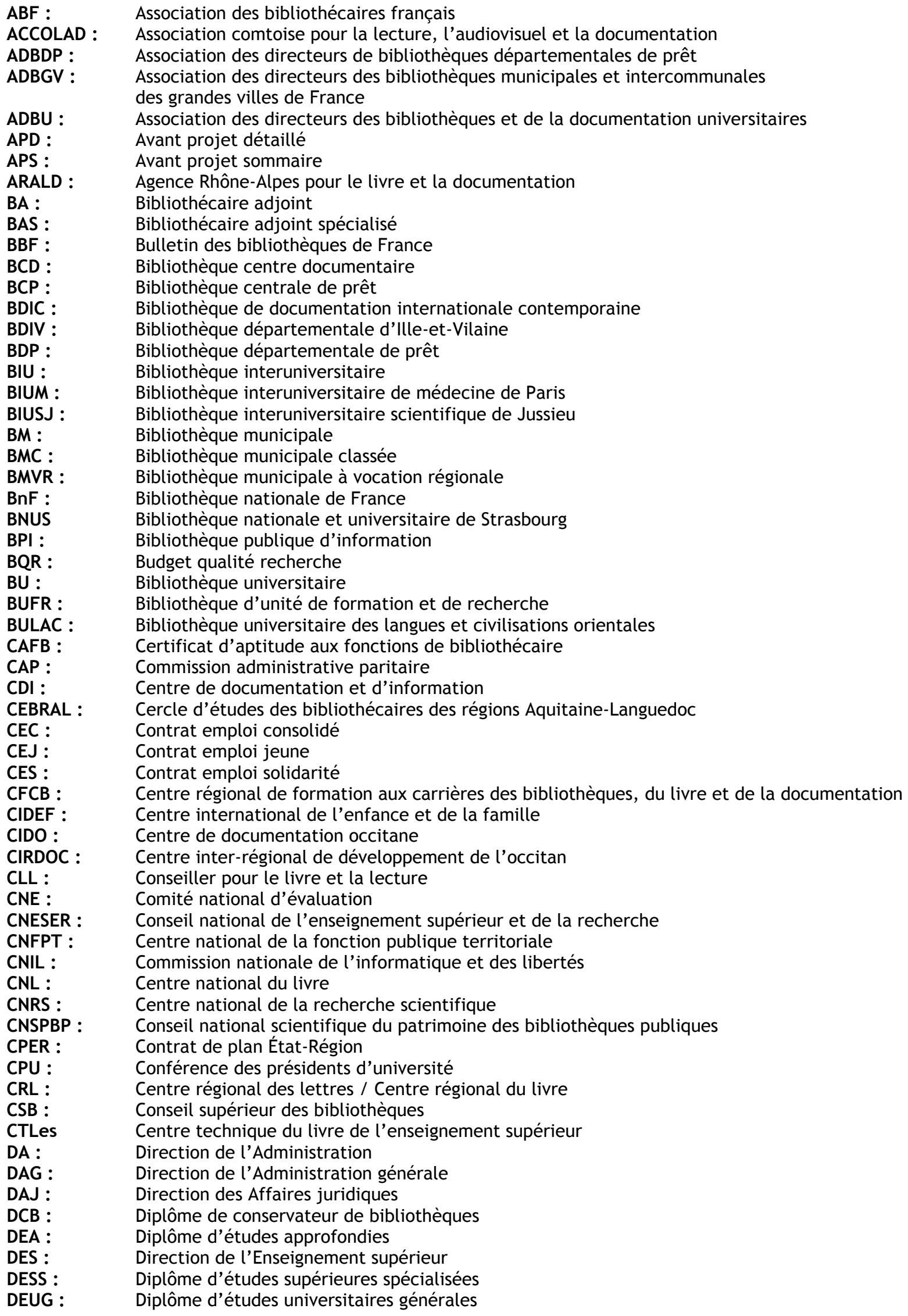


DEUST : $\quad$ Diplôme d'études universitaires scientifiques et techniques

DGC : $\quad$ Dotation générale de décentralisation

DGCL : $\quad$ Direction générale des Collectivités locales

DGF : Dotation générale de fonctionnement

DISTB : $\quad$ Direction de l'Information scientifique et technique et des Bibliothèques

DLL : $\quad$ Direction du Livre et de la Lecture

DPMA : $\quad$ Direction des Personnels, de la Modernisation et de l'Administration

DRAC : $\quad$ Direction régionale des Affaires culturelles

DSB : $\quad$ Diplôme supérieur de bibliothécaire

DUT : $\quad$ Diplôme universitaire de technologie

DVD : $\quad$ Digital Versatile Disc (disque digital à usages multiples)

ENC : $\quad$ École nationale des chartes

ENSAM : École nationale supérieure des arts et métiers

ENSSIB : $\quad$ École nationale supérieure des sciences de l'information et des bibliothèques

$\mathrm{EPCl}:$

FFCB :

Établissement public de coopération intercommunale

FPT :

Fédération française pour la coopération des bibliothèques, des métiers du livre

et de la documentation

FPT : $\quad$ Fonction publique territoriale

FRAB : $\quad$ Fonds régional d'acquisition pour les bibliothèques

GHSS : $\quad$ géographie, histoire, sciences de la société

IATOSS: Ingénieurs, administratifs, ouvriers, techniciens, de santé et sociaux

IFLA :

IGAAC :

IGAENR :

IGEN :

IGB :

INSA :

INSEE :

IP :

ISO :

LIBER

LOLF :

LSH :

MCC :

MDL :

MJENR :

NTIC :

ONG :

OPLPP :

OTAC :

PACA :

PCF :

International federation of library associations and institutions

Inspection générale de l'administration des affaires culturelles

Inspection générale de l'administration de l'éducation nationale et de la recherche

Inspection générale de l'éducation nationale

Inspection générale des bibliothèques

Institut national des sciences appliquées

Institut national de la statistique et des études économiques

Internet Protocol

International Organization for Standardization

Ligue des bibliothèques européennes de recherche

Loi d'organisation de la loi de finance

Lettres et sciences humaines

Ministère de la Culture et de la Communication

Médiathèque départementale de la Loire

Ministère de la Jeunesse, de l'Éducation nationale et de la Recherche

Nouvelles technologies de l'information et de la communication

Organisation non gouvernementale

Observatoire permanent de la lecture publique à Paris

Office territorial d'action culturelle

Provence-Alpes-Côte-d'Azur

Parti communiste français

PDC :

PRG :

QCM :

SAN :

SCD :

SCDU :

SDBD :

SIGB :

SHON :

SICD :

SIVU :

SUDOC :

UFR :

UNESCO :

U3M :

Politique de développement des collections

Paris Rive Gauche

Questionnaire à choix multiple

Syndicat d'agglomération nouvelle

Service commun de la documentation

Service commun de la documentation de l'université

Sous-direction des Bibliothèques et de la Documentation

Système intégré de gestion de bibliothèques

Surface hors œuvre nette

Service interétablissements de coopération documentaire

Syndicat intercommunal à vocation unique

Système universitaire de documentation

Unité de formation et de recherche

ZAC :

Organisation des Nations Unies pour l'éducation, la science et la culture

Universités du $3^{\text {ème }}$ millénaire

Zone d'aménagement concerté 
La première partie du rapport présente le bilan des activités du service, en fonction des missions qui lui ont été confiées durant l'année.

La seconde partie rassemble les principales observations tirées du travail d'évaluation des établissements, des rapports d'expertise et du travail de l'IGB avec les administrations.

On y trouvera en particulier :

Les principaux dénominateurs communs des inspections menées en 2003.

$>$ Le bilan des études qui ont concerné une bibliothèque ou des groupes de bibliothèques: en premier lieu l'audit de la BNF (D. Oppetit) qui a représenté une tâche particulièrement importante, puis les actions et études concernant les bibliothèques universitaires d'île-de-France (D. Renoult et $D$. Pallier), enfin un tableau de la lecture publique en Polynésie française (J.-L. Gautier-Gentès).

Deux thèmes transversaux : le patrimoine imprimé, le harcèlement moral. En effet ces sujets ont été examinés en 2003 dans différents types de bibliothèques.

$>$ Un état des lieux "Les bibliothèques territoriales et la décentralisation" (J.-L. GautierGentès). Ce travail s'appuie sur une analyse détaillée du fonctionnement et des perspectives du concours particulier identifié pour les bibliothèques au sein de la dotation générale de décentralisation.

En dernière partie sont regroupés les éléments d'information sur le cadre d'organisation de l'IGB et ses moyens de fonctionnement.

En annexe, différentes rubriques présentent :

$>$ pour 2003 :

- Des récapitulatifs de l'activité du service (annexes 1, 2, 3 et 4).

- La liste des textes relatifs à l'IGB (annexe 5).

$>$ pour 2004:

- La répartition des compétences (annexes 6 et 7).

- Les informations pratiques (annexe 8). 
Le mode de fonctionnement défini au début des années 1990 a été appliqué à nouveau en 2003. L'Inspection générale des bibliothèques a reçu un programme d'évaluation et de contrôle tant du ministère de la Jeunesse, de l'Éducation nationale et de la Recherche que du ministère de la Culture et de la Communication.

Pour la direction du Livre et de la Lecture, le contrôle, contrepartie de la décentralisation, est la priorité constante depuis la reconstitution de l'Inspection. Le programme d'inspection pour l'année est établi suivant les propositions des directions régionales des affaires culturelles et les priorités du ministère. Préparé lors d'une réunion le 30 avril, il a fait l'objet d'un courrier du directeur du 13 juin, accompagné de la liste des bibliothèques concernées. Une lettre du 3 juillet apportait une mise à jour au programme.

Les thèmes à examiner lors des missions n'ont pas été modifiés. Ce sont notamment l'application des statuts de la fonction publique territoriale, les organigrammes, les fonctions occupées par les conservateurs d'État dans les bibliothèques municipales classées, les types de services rendus au public, les partenariats de la bibliothèque visitée, l'évaluation des bâtiments récents, l'organisation du réseau des bibliothèques départementales de prêt, les relations entre le directeur de la bibliothèque et la collectivité territoriale.

Au ministère de la Jeunesse, de l'Éducation nationale et de la Recherche, le programme d'inspection a été préparé lors d'une réunion avec la DES le 25 février. Ce programme a été confirmé à l'IGB par courrier du directeur de cabinet en date du 9 avril.

Les inspections ont généralement lieu à l'occasion du renouvellement des contrats quadriennaux passés entre l'État et les universités. Une attention particulière devait être portée aux points suivants :

- définition et mise en œuvre d'une politique documentaire d'établissement

- développement de la documentation électronique et de ses accès

- services aux usagers, tant en ce qui concerne les horaires d'ouverture des services que la formation à l'information scientifique et technique

- projets immobiliers

- politique du personnel : identification des agents en situation de difficultés professionnelles, incidences des modalités d'organisation des services sur le positionnement des agents, adéquation des ressources des établissements aux missions et objectifs des services, résorption de la précarité.

L'Inspection générale des bibliothèques est invitée à continuer à seconder les efforts entrepris en faveur d'une gestion des ressources humaines plus efficace en encourageant la mobilité des agents et en signalant à l'administration les éléments aptes à exercer de plus larges responsabilités.

La date à laquelle est fixé le programme DLL a conduit au cours de ces dernières années à l'exécuter sur le second semestre d'une année et le premier semestre de l'année suivante. Ce problème de calendrier devrait être corrigé à partir de 2004.

Par ailleurs, chaque année, des missions prévues tant pour le MJENR que pour le MCC doivent être différées pour des raisons pratiques (vœu de la collectivité ou de l'université, départ du directeur de la bibliothèque, etc.). 
Le tableau ci-après indique les missions menées en 2003, correspondant à la fin du programme 2002 ou au programme 2003, ainsi que celles effectuées par les inspecteurs pour diverses raisons (présence dans la région, lien avec une inspection programmée ou un contrôle effectué précédemment, etc.).

\begin{tabular}{|c|c|c|}
\hline Établissements & Contrôles & Visites \\
\hline BIU/SICD & Jussieu & BDIC \\
\hline SCDU & $\begin{array}{l}\text { Aix-Marseille } 1 \\
\text { Angers } \\
\text { Franche-Comté } \\
\text { La Rochelle } \\
\text { Paris } 7 \\
\text { Polynésie française } \\
\text { Reims Champagne-Ardenne } \\
\text { Rennes } 1\end{array}$ & $\begin{array}{l}\text { Paris } 13 \\
\text { Grenoble } 2\end{array}$ \\
\hline BDP & Ille-et-Vilaine & Médiathèque de la Loire \\
\hline BM & $\begin{array}{l}\text { Briançon } \\
\text { Mont-de-Marsan } \\
\text { Sanary-sur-Mer } \\
\text { Sète } \\
\text { Médiathèque intercommunale } \\
\text { du SAN Ouest-Provence }\end{array}$ & $\begin{array}{l}\text { Bar-le-Duc } \\
\text { Billère } \\
\text { Chartres } \\
\text { Châteauroux } \\
\text { Épinal } \\
\text { Fontainebleau } \\
\text { Lucé } \\
\text { Mantes-la-Jolie } \\
\text { Martigues } \\
\text { Montbrison } \\
\text { Saint-Bonnet-le-Château } \\
\text { Saint-Brice-Courcelles } \\
\text { Saint-Mihiel } \\
\text { Bibliothèque de la Maison } \\
\text { de la culture de Papeete }\end{array}$ \\
\hline BMC & $\begin{array}{l}\text { Bourges } \\
\text { Caen-la-Mer (BMC Caen et } \\
\text { BM Hérouville) } \\
\text { La Rochelle } \\
\text { Le Mans }\end{array}$ & $\begin{array}{l}\text { Aix-en-Provence } \\
\text { Bordeaux } \\
\text { Dijon } \\
\text { Pau } \\
\text { Reims } \\
\text { Rennes }\end{array}$ \\
\hline & 20 & 24 \\
\hline Autres & \multicolumn{2}{|c|}{$\begin{array}{l}\text { Bibliothèque nationale de France } \\
\text { ENSSIB } \\
\text { ENSAM, bibliothèques d'Angers } \\
\text { et de Châlons-en-Champagne } \\
\text { Mise en réseau des SCD de Haute-Normandie } \\
\text { Mission expertise CIRDOC } \\
\text { Bibliothèque marxiste de Paris }\end{array}$} \\
\hline \multicolumn{3}{|c|}{51 missions } \\
\hline
\end{tabular}

En 2003, les 8 inspecteurs ont été mobilisés pour la présidence et l'organisation de l'un des concours ou examen professionnels de l'année, auxquels se sont ajoutés les concours réservés. Certains ont cumulé plusieurs responsabilités.

\subsection{Activites de CONTROLE ET d'Évaluation}

\begin{tabular}{|r|c|c|c|c|}
\hline & Programmes & $\begin{array}{c}\text { Demandes } \\
\text { spécifiques }\end{array}$ & $\begin{array}{l}\text { Visites hors } \\
\text { programme }\end{array}$ & TOTAL \\
\hline Éducation nationale & & & & \\
\hline Contrôles & 11 & 3 & & 14 \\
\hline Visites & & & 3 & 3 \\
\hline total & 11 & 3 & 3 & 17 \\
\hline Culture & & & & 13 \\
\hline Contrôles & 10 & 3 & & 21 \\
\hline Visites & 15 & & 6 & 34 \\
\hline total & 25 & 3 & 6 & 51 \\
\hline TOTAL & 36 & 6 & 9 & \\
\hline
\end{tabular}




\subsubsection{Contrôle et évaluation}

L'activité de contrôle et d'évaluation a concerné en 200316 établissements relevant du ministère de la Jeunesse, de l'Éducation nationale et de la Recherche (dont deux établissements interuniversitaires), 33 organismes relevant du ministère de la Culture et de la Communication (dont deux bibliothèques départementales de prêt) et 2 organismes extérieurs (la Bibliothèque marxiste de Paris et le Centre interrégional de développement de l'occitan, CIRDOC).

\subsubsection{Contrôles}

Pour les bibliothèques de l'enseignement supérieur, le programme concernait, pour l'essentiel, des SCD dont l'université était en phase de renouvellement de contrat.

L'inspection du SCD de l'université de Polynésie française suivait la prise de fonction d'une nouvelle directrice. directeur.

Pour le SCD d'Angers, il s'agissait de faire un bilan un an après l'arrivée d'un nouveau

La BIUSJ a été, pour l'année 2003, le seul SICD contrôlé. L'inspection se situait dans le contexte du réaménagement du campus Jussieu et la perspective d'un changement de statut entraînant le partage de la BIU. En outre, la BIUSJ et le SCD de Paris 7 n'avaient pas été inspectés depuis plus de vingt ans.

Pour les bibliothèques des collectivités territoriales, les demandes de contrôle émanent le plus souvent des DRAC.

A titre d'exemples :

- A la bibliothèque municipale de Mont-de-Marsan, l'objet de l'inspection était de dresser un état de la $B M$, dont tous les indicateurs prouvent l'insuffisance, et de conforter et orienter un projet de bibliothèque pour la communauté d'agglomération.

- La médiathèque intercommunale du Syndicat d'agglomération nouvelle Ouest-Provence, qui vient d'englober de nouvelles communes, constitue un exemple d'intercommunalité ancienne remontant aux années 70 ; elle n'avait jamais fait l'objet d'une inspection.

- La bibliothèque municipale de Sète, intercommunale depuis décembre 2003, doit trouver de nouvelles formes de travail dans ce nouveau contexte.

- Caen-la-Mer montre une forte volonté politique de passage à l'intercommunalité mais le service interétablissements de coopération documentaire, placé sous la responsabilité de la directrice de la bibliothèque de Caen, ne dispose pas encore de moyens propres.

- À Sanary-sur-mer, il s'agissait de l'évaluation d'un établissement récemment ouvert mais n'ayant plus de professionnel à sa tête.

- Pour la médiathèque de La Rochelle, il fallait analyser des problèmes de gestion du personnel et de relations avec les autorités locales et situer la bibliothèque dans un dispositif de développement de la lecture au niveau de la communauté d'agglomération.

- La bibliothèque du Mans justifiait un bilan, un an après la prise de fonctions de la nouvelle directrice ; il paraissait utile d'inciter la ville à une réflexion à moyen terme sur le développement de la lecture publique.

- Une visite d'inspection de la bibliothèque municipale de Mantes-la-Jolie a été faite pour évaluer les collections patrimoniales et proposer un programme de valorisation. 
- Quant à la bibliothèque municipale de Briançon, elle est confrontée à une situation de déshérence des collections patrimoniales, de pénurie de personnel et de locaux inadaptés.

\subsubsection{Audits}

Une inspection conjointe IGAENR-IGB à l'ENSSIB a été demandée par le cabinet du Ministre pour examiner une plainte pour harcèlement moral. Pour l'IGB, c'est $D$. Pallier qui en a été chargé.

Un audit de la Bibliothèque nationale de France en vue de la préparation d'un contrat d'objectifs et de moyens a été confié, par le directeur de cabinet du ministre de la Culture et de la Communication, à un inspecteur de l'IGAAC. Au titre de l'IGB, D. Oppetit a participé à cette mission.

Un rapport qui avait fait l'objet d'une demande spécifique du directeur de cabinet du MJENR à la fin de 2002 a été rendu en 2003. L'étude demandée, à la sollicitation du rectorat, portait sur la mise en réseau des SCD de Haute-Normandie, prévue au contrat du plan État-Région 2000-2006. Le rapport indique l'état des outils techniques des SCD et de la bibliothèque de l'INSA de Rouen. Il les situe par rapport aux réseaux documentaires actuellement en activité ou en projet dans les universités françaises ${ }^{(1)}$ et énonce un certain nombre de propositions d'action.

\subsubsection{Visites inscrites au programme du ministère de la Culture}

Les demandes de visites relèvent souvent du même type de motifs que les demandes de contrôles. Comme pour les contrôles, les thèmes essentiels sont traités en partie 2 .

- À la bibliothèque municipale de Martigues, il s'agissait d'examiner un projet d'extension et de réaménagement de la bibliothèque. La bibliothèque de Chartres a un projet d'implantation dans les anciens locaux de la Poste centrale.

- La bibliothèque municipale de Châteauroux est confrontée à de nouvelles conditions budgétaires. Celle de Lucé se trouve face à des difficultés de personnel et à une baisse de son budget ; les relations de sa direction avec la ville sont tendues.

- La visite de la bibliothèque municipale de Fontainebleau avait pour cause des problèmes de rapports avec la ville, suite notamment à un désherbage mal conduit. Celle de la bibliothèque de Saint-Brice-Courcelles avait été demandée par le maire de la commune.

- Pour la bibliothèque municipale de Reims, une visite devait précéder l'inauguration qui a eu lieu en présence du ministre de la Culture et de la Communication.

- Une visite de la bibliothèque du chapitre de Saint-Claude, suivie d'une réunion présidée par la sous-préfète, a été organisée à la demande de la DRAC de Franche-Comté. Cette rencontre avait pour objet d'entendre les conclusions d'ACCOLAD sur l'état du fonds et d'examiner avec la municipalité et le chapitre les conditions d'un transfert des collections de la cathédrale à la bibliothèque municipale.

- À Bar-le-Duc, lors de son installation dans de nouveaux locaux, la médiathèque JeanJeukens n'a pu intégrer toutes ses collections patrimoniales qui, faute de place, sont restées dans les anciens locaux à l'exception de la partie la plus ancienne. Initialement prévu, un magasin spécifique reste à construire.

- Pour la bibliothèque bénédictine de Saint-Mihiel, la conservation et la valorisation de cette bibliothèque ancienne exceptionnelle nécessitent de trouver des solutions originales pour intégrer ce patrimoine dans une économie touristique intelligente.

\footnotetext{
(1) Ces réseaux interuniversitaires régionaux ont été présentés dans le rapport annuel 2002 de l’IGB, p. 34-39.
} 


\subsubsection{Visites faites à l'initiative de l'inspecteur}

Parmi les visites faites à l'initiative de l'inspecteur, on peut mentionner les cas suivants :

- SICD de Grenoble 2 : visite à l'initiative de l'inspecteur, présent sur place à l'occasion d'un colloque. Il souhaitait mieux connaître l'organisation en départements, faire le point sur un problème d'empoussièrage provoqué par des travaux en 2002 et visiter les nouveaux locaux.

- SCD de Paris 13 : l'inspectrice a rencontré M.-A. Neuman, président de l'université de Paris 13, à propos du projet de dépôt de la bibliothèque marxiste au SCD, alors en cours de restructuration (8 avril).

- Bibliothèque de la maison de la culture de Papeete : une douzaine d'années après la mission effectuée par la DLL en 1986, il s'agissait de faire un état des lieux de la lecture publique sur le Territoire et d'émettre un avis sur les projets de celui-ci.

- Médiathèque de la Loire : la visite de la Collégiale de Saint-Bonnet-le-Château avait été demandée par le ministère de la Culture. Cependant, le fonds patrimonial de la collégiale ne pouvait pas être considéré sans prendre en compte la politique patrimoniale de la médiathèque départementale de la Loire. Le département souhaite une mise en valeur de l'ensemble du patrimoine de Saint-Bonnet sur place. Cette politique conduit la directrice de la MDL à formaliser les relations entre département et communes pour le patrimoine. Trois visites ont été menées : la médiathèque départementale de la Loire pour son secteur patrimoine, la collégiale de Saint-Bonnet et le fonds ancien de la bibliothèque municipale de Montbrison.

\subsubsection{Suivi des inspections}

Cette fonction a connu une certaine importance en 2003 :

Deux des missions menées par J.-M. Arnoult ont été des visites de suivi : suivi des projets de la BMC de Dijon, visitée en 2001 ; visite de la BM d'Épinal, inspectée en 2002.

Comme suite à l'inspection de 2002, J.-L. Gautier-Gentès a participé à une réunion ayant pour objet la restructuration du bâtiment de la BMC de Bordeaux. Cette restructuration était recommandée par le rapport de l'inspection de 2002. D'autres préconisations sont mises en oeuvre : fusion de deux annexes au profit d'un bâtiment nouveau ; rétroconversion (Bordeaux était resté en dehors du programme national).

A la suite de l'inspection de la BMC de Pau en 2002 et dans le cadre du suivi d'une bibliothèque communautaire à dimension régionale, J.-L. Gautier-Gentès a participé au jury de recrutement du chef de projet et à une rencontre avec l'assemblée communautaire (10 juillet). Sa visite de la BM de Billère, qui fait partie de la communauté d'agglomération de Pau, se situait dans la même perspective.

A la suite de la visite de la BM de Rennes, faite en 2002, L. Mérigot devait faire un point sur l'état d'avancement du chantier des Champs libres et sur le développement du réseau de lecture publique rennais.

Du côté des bibliothèques universitaires, D. Pallier a eu un entretien avec M. Falga, administrateur de la BNUS, à la demande de ce dernier, au sujet des conclusions du rapport IGA/IGB fait en 2000 sur la Bibliothèque nationale et universitaire de Strasbourg. A la suite de l'inspection de la BIUSJ, il a été invité au conseil de cette bibliothèque pour présenter cette mission. 


\subsubsection{Activités d'expertise et de conseil hors du champ d'inspection}

A. Poirot a assuré pour la DLL le suivi du dossier de l'organisation documentaire du ministère de la Culture et de la Communication. Une réunion, présidée par le directeur de l'Administration générale, a eu lieu le 17 septembre et a abouti à des propositions soumises à l'arbitrage du directeur de Cabinet. Cette activité d'expertise s'inscrit dans le droit fil du travail mené en 2001 et de la remise du rapport La fonction documentaire au sein du ministère de la Culture et de la Communication : les perspectives ouvertes par l'opération Saint-Honoré-BonsEnfants (cf. Rapport annuel 2001 de l'IGB, p. 97-100). La mise en œuvre du projet est assurée par une chargée de mission (Mme Anne Faure, conservateur de bibliothèque) qui a poursuivi la réflexion engagée, notamment par le recours à un consultant extérieur.

Une mission d'expertise a été diligentée par la direction de l'Enseignement supérieur le 26 mars, puis confirmée le 21 juin 2002, afin d'examiner les solutions les plus adaptées à la sauvegarde, au développement et à l'exploitation scientifique de la Bibliothèque marxiste de Paris. Elle devait en outre explorer la piste d'un dépôt des fonds auprès d'une bibliothèque de l'enseignement supérieur intéressée par leur mise en valeur. Pour des raisons d'accessibilité des collections, la mission, confiée à D. Oppetit, n'a pu s'effectuer que les 5 et 11 février 2003. Dans le même temps, des entretiens ont été conduits avec Dominique Coq, chef du département des politiques documentaires et patrimoniales à la direction du Livre et de la Lecture, et avec Christine de Joux, conservateur au département de la politique archivistique et de la politique interministérielle à la direction des Archives de France, destinés à préparer l'intégration des fonds manuscrits de la Bibliothèque marxiste dans la procédure de classement demandé par le Parti communiste français pour l'ensemble de ses archives historiques ${ }^{(2)}$, au titre de la protection du patrimoine historique. Ce classement a été prononcé, sur proposition de la directrice des Archives de France, lors de la réunion du Conseil supérieur des Archives du 12 mai $2003{ }^{33}$.

Le comité d'évaluation des pôles universitaires d'île-de-France a poursuivi ses travaux. D. Oppetit, au titre de l'IGB, et $D$. Renoult, en tant que chef de la mission interacadémique U3M auprès du recteur de Paris, ont collaboré aux études préparatoires à l'établissement d'un état des lieux de la vie étudiante, ou "toile de fond", portant sur les éléments suivants: patrimoine universitaire ; bibliothèques universitaires; équipements sportifs ; restauration universitaire ; logement social étudiant. Un document de synthèse, établissant la cartographie ${ }^{(4)}$ de ces différents paramètres, a été présenté et commenté lors de la réunion plénière du 17 novembre. Il doit encore être amendé et complété.

Le Centre inter-régional de développement de l'occitan (CIRDOC) est un syndicat mixte fondé par la Région Languedoc-Roussillon et la ville de Béziers. Son champ d'action est la conservation et la valorisation du patrimoine écrit, visuel et musical occitan ainsi que l'action culturelle liée à sa promotion et sa diffusion. Les fonds documentaires gérés par le CIRDOC ont pour origine les fonds donnés par le Centre de documentation occitane (CIDO) à la ville de Béziers qui met à disposition une équipe de bibliothécaires documentalistes sous la responsabilité du conservateur de la bibliothèque municipale. En collaboration avec la $\mathrm{BnF}, \mathrm{C}$. Lieber a été chargée d'une évaluation des collections, des conditions techniques de conservation et de traitement, de la compétence du personnel chargé de leur mise en valeur ; il convenait également d'examiner l'articulation possible entre le CIRDOC et la future médiathèque d'agglomération de Béziers. Le CIRDOC demande à bénéficier du dépôt légal pour les documents occitans et a posé sa candidature pour être pôle associé de la BnF pour les langues, la littérature et la civilisation occitanes.

A la demande de la directrice générale de l'École nationale supérieure des arts et métiers (ENSAM), le ministère de la Jeunesse, de l'Éducation nationale et de la Recherche a souhaité qu'une étude du patrimoine documentaire ancien conservé dans les centres d'Aix-en-Provence, d'Angers et

\footnotetext{
(2) Les archives du parti communiste elles-mêmes font l'objet d'un dépôt aux archives départementales de la Seine-SaintDenis : une convention de partenariat entre le parti communiste et le conseil général de la Seine-Saint-Denis régira les modalités de conservation et de communication des documents.

(3) Conformément aux dispositions de la loi du 3 janvier 1979 sur les archives. La direction des Archives de France, en engageant une procédure de classement de l'ensemble archives et bibliothèque, a montré que l'État prenait bien en compte la dimension historique de cet ensemble documentaire.

(4) Ce travail a été assuré par l’IAURIF.
} 
de Châlons-en-Champagne de l'ENSAM, soit conduite, accompagnée de propositions en vue d'une meilleure conservation et valorisation de ces fonds. L'étude a été engagée par J. M. Arnoult.

Dans le cadre de la Bibliothèque Méjanes, à Aix-en-Provence, J.-M. Arnoult a fait une mission d'expertise sur des collections ayant subi des infestations suite à des dysfonctionnements dans l'environnement. Il lui a également été demandé par le CNSPBP d'évaluer un atelier privé de restauration.

\subsection{ACTIVITES INTERNATIONALES}

\subsubsection{Activités internationales des membres de l'Inspection}

J.-M. Arnoult a participé à un certain nombre de programmes et réunions :

- Expertise des bibliothèques irakiennes (UNESCO)

- Réunion internationale d'experts sur le patrimoine irakien, Tokyo

- Suivi de l'aide à la reconstruction des bibliothèques irakiennes Bleu), Delft

- Opération d'aide aux bibliothèques universitaires irakiennes (Prince Claus Fund, Bouclier

- Expertise des bibliothèques afghanes

- Mise en place du programme de développement de la lecture et des bibliothèques publiques en Mauritanie, Nouakchott-Rabat (UNESCO)

- Suivi du programme de sauvegarde des manuscrits mauritaniens

- Réunion des associations des amis de la Bibliothèque d'Alexandrie, Alexandrie

- Réunion internationale du TC46 de l'ISO, Rome

J.-L. Gautier-Gentès a présenté une conférence sur le thème "Bibliothèques et décentralisation: l'expérience française" lors de la $6^{\text {ème }}$ conférence internationale Bibliothèques publiques dans la nouvelle Europe qui s'est tenue à Varsovie les 20 et 21 novembre.

A l'occasion de visites en France, à des fins d'information et de coopération, il a reçu, afin de leur présenter l'Inspection générale des bibliothèques et la situation des bibliothèques françaises :

- une délégation du ministère de la Culture mexicain conduite par le directeur général des bibliothèques (20 octobre) ;

- la directrice générale de la bibliothèque nationale d’Angola (28 novembre).

J.-L. Gautier-Gentès a participé au congrès de l'IFLA en août à Berlin et J.-M. Arnoult y a présenté une communication sur les bibliothèques irakiennes.

\subsubsection{Missions en Irak et en Afghanistan}

La communauté internationale est très attentive à la situation de ces deux pays. Il a donc paru utile de rendre accessibles dans le rapport annuel de l'Inspection les principaux constats faits par J.-M. Arnoult, lors des missions d'expertise qui lui ont été confiées.

\subsubsection{1. Évaluation de l'état du patrimoine irakien}

Dans les semaines qui ont suivi la fin des opérations militaires en Irak, l'UNESCO conduisit une première mission (17-20 mai 2003) consacrée essentiellement au patrimoine muséographique et archéologique. Une autre mission destinée à approfondir l'évaluation initiale en l'ouvrant notamment aux bibliothèques et aux archives fut programmée dans une seconde étape (27 juin-6 juillet). Elle avait pour objectif de faire un bilan de la situation des institutions culturelles irakiennes pour identifier leurs besoins urgents et pour esquisser des projets de réhabilitation et de restauration, à moyen et long termes. 
Conduite par l'adjoint du directeur général de l'UNESCO pour la culture, elle était composée de 4 archéologues, 1 architecte, 1 spécialiste de conservation, 1 représentant d'Interpol et 1 bibliothécaire.

\section{- Avant 2003}

> Dans une longue tradition savante fort ancienne, bibliothèques privées et bibliothèques publiques avaient donné de l'Irak une image de calme érudition, penchée sur la longue histoire d'un pays ouvert aux coopérations et aux évolutions. Dans les décennies 1960-1970, furent créées la plupart des universités et des grandes institutions culturelles ainsi que la Bibliothèque nationale créée en 1920 mais installée dans ses nouveaux locaux en 1977. Le Centre des manuscrits (alors déposé au Musée d'Irak) fut créé en tant qu'institution indépendante en 1988, puis complété ultérieurement par le rassemblement de petites collections publiques ou privées.

Si la guerre Iran-Irak (1980-1988) a laissé des traces dans les acquisitions, ralenties dans de nombreux établissements, c'est surtout à partir de 1991 que l'arrêt quasi total des achats de livres et des abonnements (embargo, manque de crédits) marque le début d'une longue période de stagnation. L'absence de budgets, l'abandon des projets d'informatisation, le gel des programmes de formation des professionnels et le départ de ces derniers vers d'autres activités plus rémunératrices, ont plongé progressivement la plupart des bibliothèques dans une léthargie profonde mal acceptée par les usagers. Le conflit de 2003 est venu frapper directement ces établissements par la destruction volontaire pour certains d'entre eux, et indirectement en achevant de détruire l'ensemble des structures de fonctionnement.

\section{- Les dommages subis}

Cibles directes : la Bibliothèque nationale à Bagdad - en incluant les Archives nationales -, la bibliothèque publique de Bassorah, la bibliothèque des Awqaf à Bagdad, et d'autres probablement, ont été volontairement détruites avec un souci perfectionniste de réduire en cendres les livres et de détruire les bâtiments eux-mêmes. A ce qu'on a pu juger, il y eut peu ou pas de pillages dans ces établissements : les matériels et les équipements d'une certaine valeur pouvant être revendus (photocopieurs, caméras à microfilms, climatiseurs, par exemple) ont été retrouvés à l'état de masses informes de plastique fondu ; des collections de livres (certains usuels et certains dictionnaires par exemple) ont été retrouvées à demi calcinées : elles n'ont donc pas été pillées pour être revendues sur le marché après un nettoyage des pages de titre. On est face à une volonté de destruction qui s'est donnée les moyens de ses objectifs en utilisant des techniques appropriées (lance-flammes, phosphore, etc.), dans l'esprit du vandalisme absolu qui ne s'embarrasse ni de pillage ni d'une recherche de profit immédiat.

Cibles indirectes : dans la confusion créée par les opérations militaires, les pillages les plus extrêmes ont été opérés dans les bibliothèques universitaires livrées sans protection à la recherche de tout ce qui pouvait alors être source d'argent. Organisé ou non, le pillage des équipements (climatiseurs, portes, fenêtres, tables, chaises, ordinateurs, photocopieurs, rayonnages, etc.) a été systématique à Bassorah, à Bagdad, à Mossoul, notamment dans des bibliothèques sans défense. C'est naturellement le profit qui est à l'origine de ces débordements. Mais les livres eux-mêmes n'ont été les victimes de ces pillages que dans une faible mesure : la plupart étaient en effet de revente difficile compte tenu de leur peu d'intérêt et de leur ancienneté sur les sujets traités. C'est aussi la raison pour laquelle ils ont été saccagés et en partie brûlés, probablement par dépit plus que par volonté de destruction systématique, avec des moyens d'amateurs (de simples allumettes ?) qui n'ont rien de comparable avec les moyens professionnels (lance-flammes). Et lorsque les imams des mosquées ont jeté l'anathème sur les voleurs de livres - à Mossoul par exemple - les livres ont été rapportés discrètement à la bibliothèque. Ces pillages ont eu néanmoins des conséquences immédiates sur la vie des bibliothèques qui ont vu le retour des étudiants à la rentrée universitaire 2003 sans les moyens les plus élémentaires de fonctionner, pas même les moyens d'accueillir, faute de tables, de chaises.

En conclusion provisoire, on considère que les pertes dans les bibliothèques relevant des administrations officielles sont les suivantes : la Bibliothèque nationale a perdu environ $30 \%$ de ses collections, les bibliothèques universitaires de Bagdad environ $10 \%$, la bibliothèque universitaire de Bassorah environ $20 \%$, celle de Mossoul environ 5 à $10 \%$. La bibliothèque publique de Bassorah a 
perdu $100 \%$ de ses collections ainsi que la bibliothèque du musée d'ethnographie. La bibliothèque religieuse de Bagdad (bibliothèque des Awqaf) et probablement des bibliothèques religieuses dans d'autres villes (Nassiryia, Kerbala, Najaf, etc.), ont perdu l'intégralité de leurs manuscrits et une grande partie de leurs collections de livres imprimés. Quant aux Archives nationales, le bilan est impossible à faire dans l'état de nos connaissances actuelles.

En revanche, le Centre des manuscrits, déménagé de manière opportune dans un abri antiatomique dès les premiers jours de l'année 2003, a été sauvé intégralement.

\section{- Pourquoi}

Les mêmes questions sur l'origine de ces crimes et sur leurs auteurs, posées à Bagdad et à Bassorah, n'ont pas reçu de réponses crédibles : on cherche d'abord à accabler l'adversaire sans faire d'analyse objective. La Bibliothèque nationale et les Archives nationales (celles-ci conservaient surtout des archives anciennes, les archives ottomanes, et les archives jusqu'au mandat britannique) pouvaient sans doute constituer un symbole politique du régime déchu, d'où l'acharnement manifesté : un premier incendie le 14 avril (incomplet) a été suivi d'un second incendie une semaine plus tard comme pour parachever le travail. Mais la bibliothèque publique de Bassorah pouvait-elle être considérée comme un symbole politique ? Saura-t-on jamais la vérité ?

\section{- Les bibliothèques privées}

Le sort des bibliothèques privées, quant à lui, est mal connu. Dans le cadre de la mission UNESCO, il n'a pas été possible d'examiner, faute de temps, les bibliothèques privées ou semi privées comme les bibliothèques religieuses des différentes confessions. L'Irak est un pays de collectionneurs et de bibliophiles et le nombre de bibliothèques privées est considérable. Compte tenu de la nature du conflit, il est dans la logique d'un changement de régime que ces bibliothèques, sans protection particulière, aient été pillées d'une manière organisée. On en a pour preuve la recrudescence des propositions de ventes de manuscrits dans des pays du Proche-Orient, manuscrits qui proviennent sans doute de ces bibliothèques privées irakiennes. Mais l'identification des propriétaires est problématique faute souvent de certitudes fondées sur des cachets, des exlibris et des appartenances s'appuyant sur des descriptions précises et incontestables. Une base des marques de propriété connues a été créée à l'université de Chicago dans l'intention de faciliter le travail d'Interpol pour identifier les manuscrits circulant de manière illicite. Autant avouer que ces recherches reposent sur des éléments de preuves ténues tant que les propriétaires n'auront pas euxmêmes déclarés les pillages de leurs biens au cours du conflit. Car on ne peut ignorer que certaines familles parvenues à un grand état de dénuement au cours de la décennie précédant le conflit, ont vendu pour survivre des pièces de leurs collections, et notamment des livres qui se retrouvent, en toute légalité, sur le marché international.

\section{- Conclusions}

Les bibliothèques et archives irakiennes connaissaient, depuis au moins le conflit de 1991, des difficultés de fonctionnement certaines et accusaient un retard considérable dans tous les domaines. L'embargo avait fortement amplifié les lacunes dans les acquisitions (périodiques en particulier). Les efforts pour la reconstruction des bibliothèques et archives auront pour objectif de rattraper ce retard et devront porter notamment sur quatre points :

Remise en état des locaux : les bâtiments endommagés nécessitent des travaux de restauration et d'équipement ; c'est le cas des bibliothèques universitaires. En revanche certaines bibliothèques nécessitent une reconstruction complète : Bibliothèque nationale et Archives nationales, bibliothèque publique de Bassorah, et d'autres.

Reconstruction des collections : les collections détruites doivent être reconstituées selon des programmes d'autant plus complexes à établir que les catalogues ont été endommagés ou détruits. Elles doivent aussi être complétées pour pallier les effets de l'embargo qui les a pénalisées depuis 1991. 
Formation des personnels : en réduisant les acquisitions, l'embargo a influé sur la qualification des professionnels des bibliothèques dont la présence, dans nombre de cas, n'était plus nécessaire. Il importe donc de former au plus vite des techniciens des bibliothèques, dans toutes les catégories.

Enfin, la réorganisation des administrations des ministères de la Culture et de l'Éducation (loi sur le dépôt légal, loi sur les archives par exemple) est indispensable pour assurer la cohérence du réseau reconstruit des bibliothèques ; ce dossier est certes dépendant de la réorganisation même du pays, mais il importe de ne pas oublier les bibliothèques et les archives dans cette entreprise.

\subsubsection{Collections et bibliothèques publiques afghanes}

A la demande des autorités afghanes, le ministère des Affaires étrangères a diligenté une mission d'expertise des collections patrimoniales conservées en Afghanistan. La mission comportait trois volets : les collections de manuscrits, les archives et les bibliothèques publiques. Le volet archives a été conduit par Christine Martinez, conservatrice chargée des Relations internationales à la direction des Archives de France, par Olivia Pérez, de la direction des Archives du ministère des Affaires étrangères et par J.-M. Arnoult.

Les collections patrimoniales les plus importantes sont conservées aux Archives nationales d'Afghanistan à Kaboul, installées dans un ancien palais restauré dans les années 1970 et récemment rénové par le nouveau gouvernement afghan. II s'agit d'une collection d'environ 12000 manuscrits de provenances diverses, rassemblés de manière volontariste et conservés aux Archives nationales (il n'y a pas de bibliothèque nationale en Afghanistan) ; outre des archives anciennes, une collection d'environ 1500 albums de photographies anciennes d'un intérêt considérable pour l'histoire du pays sont conservées dans les mêmes locaux.

La collection a traversé sans trop de dommages les conflits des dix dernières années, "oubliée" dans les magasins situés au sous-sol du bâtiment des Archives nationales. En dépit de l'absence de climatisation et de ventilation mécanique - l'électricité est distribuée de manière très irrégulière et imprévisible -, les conditions climatiques de Kaboul ( 1800 mètres d'altitude, températures basses en hiver, élevées en été, avec un taux d'humidité assez bas) ont permis d'éviter les problèmes d'infestation qu'on aurait pu redouter dans un milieu quasi clos pendant de nombreuses années. Dans l'état actuel, seules quelques traces d'insectes ont été repérées, mais qui ne nécessitent pas d'interventions lourdes de désinfection.

Les tâches principales sont de deux ordres : mettre en place une opération de conservation préventive (nettoyage, rangement sur les rayonnages, protection par des boîtes ou des pochettes, conditionnements appropriés notamment pour les photographies, nettoyage et remise en état de l'installation de ventilation) ; opération de catalogage à partir des catalogues anciens et des inventaires qui sont tous incomplets. Les deux opérations peuvent être menées en même temps.

Mais, dans une première phase, l'équipe des Archives nationales responsable des manuscrits a surtout besoin d'une formation pour la mise à niveau de compétences anciennes et pour acquérir des méthodes de travail conformes aux exigences actuelles.

Les bibliothèques publiques afghanes ont beaucoup souffert des conflits. Des 74 établissements officiellement recensés dans les années 80 , il n'en reste plus que 54 , sans certitude, aucun bilan exact n'ayant été réalisé depuis 2002. Seules les bibliothèques de Kaboul sont connues avec quelque précision : la Bibliothèque publique centrale et ses 5 annexes (dont une seule serait ouverte, l'une ayant été transformée en prison). Le directeur, qui a les fonctions de directeur des bibliothèques afghanes, ne connaît guère en fait que ce qui est dans la capitale. Faute d'avoir pu visiter d'autres villes, on est contraint de s'en remettre aux informations non vérifiées collectées par des ONG qui ont une vision pessimiste de l'état des bibliothèques dans le reste du pays.

La Bibliothèque publique centrale de Kaboul dispose de toutes les sections d'une bibliothèque publique habituelle (enfants, adolescents, adultes, étude) ; ses collections se composent d'environ 150000 volumes, pour la plupart anciens et d'un intérêt limité pour les lecteurs d'aujourd'hui qui sont d'ailleurs peu nombreux (1 616 cartes avaient été enregistrées en janvier 2004, dont beaucoup d'étudiants). Un "Internetcafé" a été installé par l'UNESCO : c'est la 
section la plus fréquentée de toute la bibliothèque. En l'absence de bibliothèque nationale et de dépôt légal, la Bibliothèque publique rassemble comme elle peut, dans un service spécifique, des publications concernant l'Afghanistan.

Le personnel (environ 65 personnes dont 45 “permanents") n'a aucune qualification (seul le directeur adjoint a été formé en Corée, en URSS et en Iran).

Les difficultés de fonctionnement - outre l'irrégularité de l'alimentation électrique et l'absence de chauffage en hiver - tiennent au manque de crédits pour acquérir des livres et à l'absence de personnels qualifiés. Les dons de livres proviennent essentiellement de pays anglophones ; si la langue anglaise est la seconde langue étrangère parlée (après l'arabe, le français venant en $3^{\text {ème }}$ position), elle reste peu commune chez les usagers potentiels de la bibliothèque publique. Les seuls dons aisément accessibles sur le plan linguistique viennent d'Iran et ne sont pas les plus nombreux a priori.

Le dénuement des divers services, l'équipement archaïque, la pauvreté des collections offertes aux usagers rendent plus amer encore le fatalisme des quelques professionnels ou personnes dévouées.

On a pu constater également que la communauté internationale ne s'est pas mobilisée pour les bibliothèques afghanes. A l'exception de l'Allemagne et de l'Iran, aucun autre pays ne s'est engagé, à ce jour, pour reconstruire et développer les bibliothèques après les conflits. L'Allemagne s'est engagée dans un programme de formation et d'aide technique (mobilier notamment), à la Bibliothèque publique et à la Bibliothèque universitaire. L'Iran s'est également engagé dans un programme de dons de livres en langue farsi (l'une des langues afghanes avec le pachtoune et le dari). Ces aides ne concernent malheureusement que les bibliothèques de Kaboul. Dans les provinces, tout reste à faire.

Dans l'hypothèse où la France apporterait son aide, les domaines où l'urgence est la plus grande sont les bibliothèques publiques (formations), le secteur patrimonial (conservation et catalogage), et l'ouverture de deux dossiers importants même si l'urgence est moindre : création d'une bibliothèque nationale et mise au point d'une législation pour organiser le dépôt légal des productions afghanes afin de préserver le patrimoine contemporain. 


\subsection{Participation À des instances SCIENTIfiQUes et TeChNiQUeS}

\subsubsection{Conseils, comités et commissions}

\begin{tabular}{|l|l|}
\hline Inspecteurs & Instances \\
\hline J.-M. Arnoult & Membre du Conseil national scientifique du patrimoine des bibliothèques publiques (comité de \\
& restauration) \\
\hline & Membre du Conseil d'administration de la BNUS \\
\hline & Membre du Comité d'orientation du Centre des manuscrits d'Avranches \\
\hline & Membre du Comité d'orientation des programmes de reproduction de l'IRHT \\
\hline & Président du Comité technique "Information et documentation" de l'ISO (TC 46) \\
\hline & Membre du Comité permanent "Construction des bibliothèques" de l'IFLA \\
\hline J.-L. Gautier-Gentès & Membre du conseil d'administration de l'École nationale des chartes \\
\hline & Membre du conseil d'administration de l'ENSSIB \\
\hline & Président du conseil de perfectionnement de la formation continue (ENSSIB) \\
\hline L. Mérigot & Président du conseil scientifique du BBF \\
\hline D. Oppetit & Membre du Conseil d'administration de Médiadix \\
\hline D. Pallier & Présidente du conseil de perfectionnement du DCB (ENSSIB) \\
\hline & Membre du conseil d'administration du CTLes \\
\hline A. Poirot & Membre du Comité scientifique du Corpus iconographique de l'histoire du livre \\
\hline & Membre du Conseil scientifique de l'École nationale des chartes \\
\hline D. Renoult & Président du Conseil de perfectionnement de la formation des bibliothécaires \\
\hline & Chef de la mission interacadémique U3M auprès du recteur de Paris \\
\hline
\end{tabular}

ENSSIB

\section{- Conseil de perfectionnement du DCB}

Lors de la première réunion du nouveau conseil de perfectionnement (2 juin), le directeur de l'ENSSIB a présenté le projet de mastère selon la maquette adoptée par la commission pédagogique du 17 avril. Cette maquette devait être validée en fin d'année par le conseil d'administration de l'École afin qu'elle soit soumise à habilitation auprès du ministère dès les premiers mois de 2004 pour une ouverture à partir de septembre 2004, objectif des universités et Grandes Écoles de Lyon. Après la présentation du dispositif actuel de formation des conservateurs et des conclusions du précédent conseil de perfectionnement, R. Bérard, directeur des études, a expliqué les enjeux et les difficultés de l'intégration de la formation professionnelle dans un mastère : le mastère est à la fois un grade et un diplôme ( 3 grades : licence, mastère et doctorat). L'école a fait le choix du diplôme, diplôme nouveau qui intègre l'ensemble des formations préparées à l'ENSSIB. Cette formation est à la fois destinée aux bibliothécaires, aux conservateurs de bibliothèques, mais aussi au secteur privé (documentalistes d'entreprise).

D. Oppetit, présidente du conseil, a fait part d'une préoccupation sur la finalité même de l'école : l'enjeu de l'école est de former de jeunes diplômés à un métier (ou de mettre à niveau des agents de la fonction publique pour les candidats internes) ; or l'objectif de faire entrer cette formation dans un mastère ne doit pas faire passer au second plan l'amélioration de la formation professionnelle des élèves. Une difficulté importante pourrait venir de la similitude de formation des bibliothécaires et des conservateurs durant les deux premiers semestres (bibliothécaires : 1 an conservateurs : 18 mois) au risque d'aggraver le malaise du corps des bibliothécaires. En outre, une différence majeure a été soulignée entre les bibliothécaires territoriaux, qui ont vocation à être directeurs d'établissements et les bibliothécaires d'État qui n'ont pas a priori ce type de responsabilité. 


\section{- Conseil de perfectionnement de la formation des bibliothécaires}

A. Poirot préside ce conseil qui s'est réuni le 28 mai.

\section{- Conseil de perfectionnement de la formation continue}

Présidé par J.-L. Gautier-Gentès, le conseil de perfectionnement de la formation continue de l'ENSSIB, qui s'est réuni 2 fois ( 8 juillet et 15 décembre), a décidé de se saisir des thèmes suivants : le développement d'une culture de la formation ; validation des acquis de l'expérience et formation continue ; le service de la formation continue comme centre de ressources.

\section{- Commission pédagogique de l'ENSSIB et mastère}

Conformément à des recommandations de J.-L. Gautier-Gentès et D. Pallier précédents présidents des conseils de perfectionnement du $D C B$ et de la formation des bibliothécaires, les présidents et vice-présidents des conseils de perfectionnement sont désormais représentés à la commission pédagogique de l'ENSSIB. Ainsi D. Oppetit, A. Poirot et J.-L. Gautier-Gentès ont-ils assisté à la séance que cette commission a tenue le 17 avril et dont l'objet était le futur mastère. Ils ont également participé à un séminaire organisé par l'école les 6 et 7 octobre.

L'IGB a assisté, à la demande du directeur du Livre, à une réunion suscitée par celui-ci sur le thème du mastère avec des représentants de la BnF, de la BPI et de la médiathèque de La Villette (J.-L. Gautier-Gentès, 26 septembre).

- CA de l'ENSSIB

Le CA de l'ENSSIB a eu lieu les 14 janvier, $1^{\text {er }}$ avril et 3 juillet. L'IGB a été représentée par J.-L Gautier-Gentès.

\section{Autres conseils}

Le CA de l'École nationale des chartes s'est réuni les 30 juin et 8 décembre. J.-L. GautierGentès y représente l'Inspection.

En 2001, A. Poirot a été renouvelé comme membre du Conseil scientifique de l'École nationale des chartes. Celui-ci s'est réuni le 15 mars 2003. Parmi les sujets débattus se situe la question de la thèse de l'ENC dans le paysage de l'enseignement supérieur français et européen.

D. Pallier est membre du comité scientifique du Corpus iconographique de l'histoire du livre, qui appuie la publication d'ouvrages mettant en valeur le patrimoine imprimé des bibliothèques d'enseignement supérieur. Il a participé à la réunion du Corpus, le 20 juin, à la Bibliothèque de la Sorbonne.

Membre du conseil d'administration du CTLes, il a participé aux réunions des 28 mai et 10 décembre, présidées par $\mathrm{D}$. Renoult.

L'Inspection est régulièrement conviée à assister, en qualité d'invitée, aux réunions bisannuelles des directeurs de l'OPLPP ; J.-L. Gautier-Gentès l'a représentée à la séance qui s'est tenue le 23 juin.

\subsubsection{Groupes de travail}

$>$ DES

Les inspecteurs ont été invités à la réunion annuelle des directeurs des bibliothèques des établissements d'enseignement supérieur (28 et 29 janvier).

J.-M. Arnoult est membre de la commission technique pour la BNU de Strasbourg. 
D. Pallier a représenté le doyen à une réunion de travail avec le département de l'Économie du livre à propos des décrets d'application de la loi du 18 juin 2003 relative à la rémunération au titre du prêt en bibliothèque (examen des catégories de bibliothèques accueillant du public pour le prêt) ; cette réunion a eu lieu à la DLL le 12 septembre.

A. Poirot et C. Lieber participent au "Groupe de travail sur les missions régionales des grandes bibliothèques en région". Ce groupe s'est réuni les 18 novembre 2003 et 21 janvier 2004 ; son activité se poursuivra en 2004.

L. Mérigot et A. Poirot ont assisté au séminaire de la DLL sur la LOLF (21 janvier). A. Poirot a participé au groupe de travail sur le séminaire DLL / CLL de Toulouse sur l'économie du livre (juin).

\section{DPMA}

La DPMA ayant remis en chantier la rédaction de référentiels pour les métiers des bibliothèques, annoncée il y a plusieurs années par la direction précédemment en charge des personnels concernés, un comité de pilotage comprenant des représentants de la DPMA, de la DES, de la DLL, de l'ADBU et le doyen de l'IGB a été mis en place à la fin de l'année ( 5 décembre). Il a été décidé de constituer trois groupes de travail, respectivement pour les fonctions scientifiques et d'encadrement supérieur, pour les fonctions techniques et d'encadrement intermédiaire et pour les fonctions de magasinage. Les deux premiers groupes de travail sont présidés par des IGB, respectivement $D$. Oppetit et $A$. Poirot ; une IGB, F. Lemelle, participe au troisième.

F. Lemelle, C. Lieber et D. Pallier ont participé à la réunion DPMA/IGB, le 4 avril, sur les projets de modifications à apporter au statut du corps des bibliothécaires et à l'organisation du concours externe de recrutement des bibliothécaires. D. Pallier avait transmis des propositions écrites détaillées sur l'évolution du concours de recrutement de bibliothécaires, en prenant en compte les rapports des jurys et la réflexion du conseil de perfectionnement de la formation des bibliothécaires. La réunion a également traité du personnel de magasinage spécialisé (statut, gestion, recrutement des magasiniers en chef).

D. Pallier a participé à la réunion École des chartes/DPMA/SDBD/IGB sur l'organisation et le calendrier du concours de recrutement des élèves de l'École des chartes à l'ENSSIB, le $1^{\text {er }}$ avril.

La DPMA a mis en place un groupe de liaison avec l'ADBU. L'IGB y est représentée par le doyen. La première réunion a eu lieu le 10 décembre.

$>\mathrm{CPU}$

Le doyen a représenté l'IGB au colloque annuel de la CPU, qui s'est tenu les 20 et 21 mars à Poitiers sur le thème "Les personnels dans l'université du XXI ${ }^{\text {ème }}$ siècle : missions, métiers, partage de responsabilités".

\section{$>$ Autres réunions}

L. Mérigot a participé au club des documentalistes de l'administration.

D. Pallier a assisté au colloque organisé par la Fédération française pour la coopération des bibliothèques, des métiers du livre et de la documentation (FFCB), l'Agence Rhône-Alpes pour le livre et la documentation (ARALD), les bibliothèques municipales de Grenoble, le conservatoire national de région et "Musique et mémoire", les 14 et 15 octobre à Grenoble. Il a présenté l'IGB à la CLL de Guyane le 15 mai. 


\subsection{ACTIVITES ADMINISTRATIVES}

\subsubsection{Jurys de concours et d'examens}

\begin{tabular}{|c|c|c|c|c|c|c|c|c|}
\hline \multirow[t]{2}{*}{ Concours } & \multirow[t]{2}{*}{ IG } & \multicolumn{3}{|c|}{$\begin{array}{l}\text { Nombre de } \\
\text { postes offerts }\end{array}$} & \multirow{2}{*}{$\begin{array}{c}\text { Candidats } \\
\text { inscrits }\end{array}$} & \multicolumn{3}{|c|}{ Candidats présents } \\
\hline & & Total & Ext. & Int. & & Total & Ext. & Int. \\
\hline ENSSIB - Chartistes & D. Pallier, président & 15 & 15 & & 20 & 18 & 18 & \\
\hline Bib. adjoints spécialisés & L. Mérigot, présidente & 221 & 95 & 126 & 645 & 508 & 349 & 159 \\
\hline $\begin{array}{l}\text { Concours réservés organisés au } \\
\text { titre de la loi Sapin }\end{array}$ & & \multicolumn{3}{|c|}{$\begin{array}{l}\text { Nombre de } \\
\text { postes offerts }\end{array}$} & $\begin{array}{l}\text { Candidats } \\
\text { inscrits }\end{array}$ & \multicolumn{3}{|c|}{ Candidats présents } \\
\hline Bibliothécaires & A. Poirot, président & \multicolumn{3}{|c|}{9} & 33 & \multicolumn{3}{|c|}{33} \\
\hline \multicolumn{9}{|l|}{ Magasiniers spécialisés } \\
\hline Université de Paris 3 & $\begin{array}{l}\text { J.-L. Gautier-Gentès, } \\
\text { président }\end{array}$ & & & & & & & \\
\hline Examens professionnels & & \multicolumn{3}{|c|}{$\begin{array}{c}\text { Nombre de } \\
\text { postes offerts }\end{array}$} & $\begin{array}{l}\text { Candidats } \\
\text { inscrits }\end{array}$ & \multicolumn{3}{|c|}{ Candidats présents } \\
\hline $\begin{array}{l}\text { Assistants des bibliothèques } \\
\text { de classe exceptionnelle }\end{array}$ & F. Lemelle, présidente & \multicolumn{3}{|c|}{27} & 66 & \multicolumn{3}{|c|}{65} \\
\hline
\end{tabular}

Filière bibliothèques, État

D. Oppetit a participé à la commission de validation de acquis des élèves conservateurs de la promotion DCB 12 (ENSSIB, 4 février).

A. Poirot assure le présidence de la commission scientifique de validation des acquis pour les bibliothécaires qui ont commencé leur scolarité à l'automne 2003 (ENSSIB, 7 novembre). La session 2004 des concours externe et interne des bibliothécaires d'État a déjà fait l'objet d'une réunion du jury (6 novembre) ; les épreuves écrites devaient se dérouler le 24 février 2004.

A la demande de l'université de Paris 3, J.-L. Gautier-Gentès a présidé le jury chargé de recruter des magasiniers pour les trois bibliothèques dépendant de cette université, à savoir, outre le SCD de celle-ci, la bibliothèque Sainte-Geneviève et la bibliothèque interuniversitaire des langues orientales (7-9 janvier).

Plusieurs jurys ont été organisés pour l'intégration de travailleurs handicapés :

- Assistants des bibliothèques agent.

C. Lieber a présidé deux jurys, à Paris, en mai et en novembre, chacun concernant un

- Magasiniers spécialisés

F. Lemelle a présidé deux jurys, l'un pour deux agents à Paris, le 26 février, et l'autre pour un agent à Fort-de-France, le 9 avril.

\section{Filière bibliothèques, FPT}

A la demande du CNFPT, J.-L. Gautier-Gentès a présidé le jury du concours réservé de conservateur territorial des bibliothèques (28 mars). 


\section{Autres filières}

Un nouveau corps de conseillers et inspecteurs de la création, des enseignements artistiques et de l'action culturelle ayant été créé, J.-L. Gautier-Gentès a fait partie, à la demande du ministère de la Culture, du jury du concours interne exceptionnel pour la spécialité livre (12 mars).

\subsubsection{Gestion et évaluation des personnels}

\section{$>$ Commissions administratives paritaires}

\begin{tabular}{|l|l|l|}
\hline \multicolumn{1}{|c|}{ CORPS } & \multicolumn{2}{c|}{ IG } \\
\hline Conservateurs généraux & Jean-Luc GAUTIER-GENTĖS & (expert) \\
\hline \multicolumn{1}{|c|}{ CORPS } & \multicolumn{1}{|c|}{ TITULAIRES } & \multicolumn{1}{c|}{ SUPPLÉANTS } \\
\hline Conservateurs & Jean-Luc GAUTIER-GENTĖS & Danielle OPPETIT \\
\hline Bibliothécaires & Albert POIROT (expert) & \\
\hline BAS & $\begin{array}{l}\text { Lydia MÉRIGOT } \\
\text { Denis PALLIER }\end{array}$ & Claudine LIEBER \\
\hline Assistants des bibliothèques & $\begin{array}{l}\text { Claudine LIEBER } \\
\text { Lydia MÉRIGOT }\end{array}$ & $\cdots$ \\
\hline Magasiniers en chef & Jean-Marie ARNOULT & -...- \\
\hline Magasiniers spécialisés & Françoise LEMELLE & Claudine LIEBER \\
Denis PALLIER
\end{tabular}

Les inspecteurs sont invités à participer à des réunions préparatoires aux CAP des conservateurs : le 25 avril avec la DLL, le 28 avril avec la SDBD ; le 7 novembre avec la SDBD et le 10 novembre avec la DLL.

Outre sa participation aux deux séances annuelles, à titre de membre ou d'expert, l'IGB a participé à plusieurs séances de CAP siégeant en formation disciplinaire: conservateurs, 5 décembre (J.-L. Gautier-Gentès) ; magasiniers, 4 février (D. Oppetit).

Il est parfois demandé à un inspecteur de se saisir, en vue d'étude ou de médiation, du cas de certains agents.

\section{Commissions d'affectation}

J.-L. Gautier-Gentès a participé à la réunion d'affectation des élèves de l'ENSSIB (23 mai).

D. Pallier a représenté l'IGB à la réunion d'affectation des BAS, des assistants de bibliothèque et des magasiniers en chef (8 juillet).

A. Poirot a participé à la commission d'affectation des bibliothécaires (10 septembre).

\section{$>$ Commissions de sélection}

D. Oppetit a participé à l'entretien de sélection du conseiller pour le livre de la région Picardie (7 avril). A. Poirot est intervenu de façon similaire pour la sélection du conseiller pour le livre de la région Centre (15 octobre).

\subsubsection{Collaboration avec d'autres instances de conseil, d'évaluation et de contrôle}

Le doyen a reçu, à sa demande, J.-F. Jacques, nouveau secrétaire général du CSB, dans le cadre de la mission de réflexion confiée à celui-ci sur l'avenir du conseil ( $1^{\text {er }}$ octobre).

Au ministère de l'Éducation nationale, le doyen de l'IGB a rencontré le délégué général du Comité national d'évaluation (4 juin). 
Par ailleurs, une visite du doyen de l'IGB au doyen de l'IGEN et au chef du service de l'IGAENR (2 juin), à son initiative, a eu pour objet d'échanger des informations sur le fonctionnement des trois services et d'envisager des modes de collaboration renforcée.

Dans cette perspective, l'IGB a été invitée à participer au séminaire tenu par l'IGEN et l'IGAENR du 8 au 10 septembre. Il était prévu que les IGB participent aux groupes de travail IGENIGAENR :

- Enseignement supérieur (D. Pallier)

- Lycées (A. Poirot)

- Enseignement obligatoire (L. Mérigot)

Des inspecteurs ont assisté aux ateliers suivants :

- "Comparaison des pratiques respectives de contrôle, de conseil, d'évaluation, d'audit", (J.L. Gautier-Gentès, D. Pallier)

- "Quelle documentation pour quels besoins ?"

A la demande des deux inspections et de la documentaliste, L. Mérigot a visité, le 6 octobre, le centre de documentation du 107 rue de Grenelle. Les objectifs étaient, outre d'en prendre connaissance, d'étudier la possibilité d'une coopération, en particulier pour le prêt éventuel de documents (essentiellement de revues) et du regroupement en un lieu unique de l'ensemble des rapports des inspections.

Actuellement, le centre ne couvre qu'à la marge les thèmes et les domaines d'action de I'IGB ; il est possible toutefois de faire des copies d'articles et d'emprunter des ouvrages.

Par ailleurs, L. Mérigot a été contactée par Anne-Marie Bardi (IGEN) et Gérard Saurat (IGAENR). Ils ont examiné ensemble les points qui pouvaient s'insérer dans la thématique déjà définie et faire l'objet d'un travail commun.

Autre contact également à la suite de l'atelier, M. Jean-Louis Durpaire, inspecteur général des établissements et de la vie scolaire, a rencontré L. Mérigot afin de présenter une étude qu'il pilote sur la politique documentaire des établissements, et qui s'appuiera sur l'enquête réalisée par chaque IGEN dans son académie. Il a également évoqué la possibilité d'inspections communes.

Au ministère de la Culture, une coordination plus étroite est à l'ordre du jour entre l'Inspection générale de l'administration des affaires culturelles et les inspections spécialisées. C'est dans cette perspective que l'IGAAC (R. Klein, J.-R. Marchand) a souhaité rencontrer le doyen de l'IGB (25 février).

L'audit de la BnF, souhaité par le ministre de la Culture et de la Communication, a été conduit conjointement par l'IGAAC (R. Klein) et l'IGB (D. Oppetit). 


\subsection{AUTRES ACTIVITES SCIENTIFIQUES ET PROFESSIONNELLES}

\section{$>$ Formation}

\section{-J.-M. Arnoult}

Exposé sur "L'organisation de la conservation dans les bibliothèques" (dans le cadre de la maîtrise conservation des biens culturels de l'université Paris 1).

\section{- J.-L. Gautier-Gentès}

A la fin de la première année, les élèves de l'École nationale des chartes effectuent par groupes, dans des villes différentes, une visite de plusieurs institutions documentaires et culturelles (archives, inventaire général, musées, bibliothèques territoriales et universitaires, etc.). Il s'agit en particulier de leur permettre d'appréhender la réalité du métier de conservateur. A l'issue de ces visites, ils rédigent un rapport présenté devant une commission dans laquelle figurent, outre la directrice et des enseignants de l'école, l'Inspection générale des archives et l'Inspection générale des bibliothèques. J.-L. Gautier-Gentès a représenté celle-ci à la commission qui s'est réunie le 16 janvier.

Le 17 janvier, il a participé à l'ENSSIB, en qualité de président du conseil de perfectionnement du $\mathrm{DCB}$, à une rencontre-débat sur "le rôle, les missions et les grands enjeux du métier de conservateur" dans le cadre du séminaire d'ouverture, consacré aux politiques culturelles et scientifiques, de la scolarité de la promotion du DCB 12. formation.

Au cours de l'année, il a reçu à divers titres plusieurs élèves de différents établissements de

\section{- C. Lieber}

"Internet et les bibliothèques. Quel impact sur les usages ?". Exposé prononcé à l'occasion des journées de rencontre organisées par le ministère des Affaires étrangères pour les directeurs de médiathèques françaises à l'étranger (26 mars).

"Les outils de la recherche: utilisation et ressources des bibliothèques françaises". Intervention en langue anglaise à l'intention des boursiers Fulbright 2004 devant conduire une recherche en France.

Elle a participé au comité de pilotage BPI, chargé de suivre le projet "désherbage" d'un groupe d'élèves conservateurs dans le cadre de leur mémoire (27 mars, 20 juin).

- D. Pallier

Interventions lors des journées de formation organisées par le département de la formation continue de l'ENSSIB et le SCD de Metz, du 13 au 15 mai, consacrés à l'organisation fonctionnelle des SCD (E6- Mettre en œuvre une organisation transversale en SCD) :

"L'organisation fonctionnelle des SCD" (rappel du contexte statutaire, panorama des modes d'organisation des SCD aujourd'hui : de l'organisation en sections à l'émergence de structures transversales, perspectives d'évolution)

"Le cas de Metz : quels sont les éléments qui ont conduit l'Inspection à proposer un changement d'organisation ?"

- D. Renoult

"Les Réseaux documentaires", cours au DESS Édition, documentation, information, université de Cergy Pontoise. 


\section{Exposés, tables rondes}

- J.-M. Arnoult

"Assessment of Iraqi libraries", communication présentée au Congrès de l’IFLA, Berlin

"Les bibliothèques irakiennes après les conflits", BNF

"Bilan des bibliothèques irakiennes", Bouclier Bleu

"La conservation du patrimoine écrit mauritanien, les bibliothèques publiques de manuscrits (Chinguetti et Ouadane)", Assemblée parlementaire de la francophonie, table ronde sur la conservation du patrimoine bâti.

\section{- J.-L. Gautier-Gentès}

"Intercommunalité et bibliothèques", lors de la journée d'étude sur ce thème organisée le 3 février à Longvic par le groupe Bourgogne de l'ABF.

"Bibliothèques municipales : pour une refondation", lors de la table ronde organisée le 24 mars au salon du livre par le BBF sur le thème de la baisse de la proportion des usagers inscrits dans les bibliothèques municipales.

"Éléments pour une déontologie", lors de la journée d'étude organisée le 17 mai à Bédarieux (Hérault) par le CEBRAL sur le thème "Entre dire et faire : valeurs et déontologie professionnelles".

"Bibliothèques en crise : comment former des bibliothécaires aptes à relever les défis du changement ?", lors de la journée d'étude organisée le 26 mai à Lyon par le groupe Rhône-Alpes de l'ABF sur le thème de l'évolution du métier de bibliothécaire.

"Actualité(s) en bibliothèque et citoyenneté", lors de la journée d'étude organisée le 19 juin à Rennes par l'Agence de coopération des bibliothèques et centres de documentation en Bretagne sur le thème "Information du citoyen en bibliothèque".

En outre, lors du congrès de l’ABF (Aubagne), J.-L. Gautier-Gentès a animé, le 14 juin, la table ronde consacrée aux codes déontologiques étrangers.

- C. Lieber

Séminaire FFCB à Toulouse, en partenariat avec le CRL Midi-Pyrénées (13 et 14 novembre). Ce séminaire avait pour objet d'analyser l'évolution des missions des structures régionales pour le livre et leurs adéquations avec les objectifs et les enjeux de la décentralisation dans le cadre du projet de modification de la loi. Participation au titre de l'Inspection générale des bibliothèques à la "Table ronde sur la perception de la place et du rôle des structures régionales pour le livre dans un contexte décentralisé."

- L. Mérigot

Congrès i-expo (17-19 juin 2003) : présidence de la session "Un nouveau mode d'accès à l'information scientifique : le libre accès (open access)".

- A. Poirot

"Missions et fonctions attendues des bibliothécaires : observations d'un inspecteur général des bibliothèques", Séminaire annuel des CLL et de la DLL sur la formation (Nantes, 22 et 23 mai).

"La conservation: une idée à partager", dans le cadre des Journées d'étude sur le patrimoine organisées par ACCOLAD (Salins, 15 et 16 décembre). 
- D. Renoult

Intervention au colloque de l'Association des Archivistes tenu à la Sorbonne en janvier 2003 : "La bibliothéconomie et l'archivistique : deux disciplines au service du savoir".

Présentation d'une conférence : "Les savoirs à l'heure de la numérisation". Université de Cergy-Pontoise, 6 mars 2003.

\section{Recherche, publications}

Président du conseil scientifique du BBF, J.-L. Gautier-Gentès a présidé la séance annuelle (25 mars)

D. Renoult a suivi comme rapporteur général les travaux de l'instance chargée par le Commissariat au Plan d'évaluer la politique de contractualisation des universités. Présidée par Armand Frémont cette instance a rendu son rapport au ministre de la Jeunesse, de l'Éducation nationale et de la Recherche en novembre 2003. Il devrait être publié en février 2004 par la Documentation française.

\section{$>$ Relations avec les associations professionnelles}

Plusieurs inspecteurs ont assisté au congrès de l'Association des directeurs de bibliothèques universitaires (Limoges, 11-13 septembre). C. Lieber a participé au congrès de l'Association des bibliothécaires français, L. Mérigot aux journées d'étude de l'ADBDP et A. Poirot aux Journées d'étude annuelles de l'Association des directeurs des bibliothèques des grandes villes (ADBGV) (Tours, 17-18 mars).

\section{$>$ Autres}

- L. Mérigot et D. Pallier ont visité le centre de ressources documentaires multimédias (CRDM) commun au ministère des Affaires sociales, du Travail et de la Solidarité et au ministère de la Santé, de la Famille et des Personnes handicapées, le 5 mars.

- C. Lieber a représenté l'Inspection aux Dix ans du Carré d’Art à Nîmes (5 mai). 


\subsection{OBSERVATIONS GENERALES}

\subsubsection{Les groupes de travail}

Dans la présentation des travaux de l'Inspection en 2003, il convient de souligner la part des groupes de travail. On a donné plus haut (1.3.2) la liste de ces groupes. Leurs thèmes appellent un double commentaire.

- L'Inspection a depuis longtemps un rôle important en matière de personnel. Elle participe au recrutement des personnels des bibliothèques. Elle est présente aux conseils d'administration de l'ENSSIB et de l'École des chartes. Elle est associée à la gestion des personnels (commissions d'affectation, CAP).

Au cours des dernières années, à la demande de la direction des Personnels, de la Modernisation et de l'Administration (DPMA) et de l'ENSSIB, I'IGB s'est encore plus fortement impliquée dans les études concernant la formation et la gestion des personnels. Trois inspecteurs généraux sont présidents de conseils de perfectionnement de l'ENSSIB (respectivement pour le $D C B$, la formation des bibliothécaires et la formation continue). Ils ont participé à la commission pédagogique de l'École et aux réunions sur le projet de mastère de l'ENSSIB.

Deux inspecteurs généraux sont responsables de groupes de travail créés par la DPMA pour la rédaction des référentiels des métiers des bibliothèques. L'IGB est représentée dans le troisième groupe. Elle est en outre constamment représentée au groupe de liaison avec l'ADBU mis en place par la DPMA.

- L'Inspection est systématiquement associée aux groupes de travail constitués auprès de la direction du Livre et de la Lecture et à ses études (groupes sur l'évaluation des bibliothèques et sur la réforme de la dotation générale de décentralisation, en 2002 ; groupe sur la coopération et la fonction régionale des grandes bibliothèques municipales, plan d'action pour le patrimoine écrit, en 2003). Elle participe aux réunions préparatoires aux séminaires organisés par la DLL avec les conseillers pour le Livre et la Lecture des DRAC.

En 2003, la sous-direction des Bibliothèques et de la Documentation de la direction de l'Enseignement supérieur n'a appelé l'Inspection à participer à aucun groupe de travail, à l'exception d'une commission technique pour la Bibliothèque nationale et universitaire de Strasbourg.

\subsubsection{Les concours}

Dans le rapport 1998, puis dans le rapport 2002, l'Inspection a souligné la place prise par l'organisation des concours dans son activité. L'organisation par l'Inspection du recrutement des personnels de bibliothèques est une lourde charge, qui mérite un bilan annuel.

Depuis plusieurs années, l'IGB a la responsabilité de la totalité des concours de recrutement et examens professionnels des personnels des bibliothèques, en ce qui concerne les corps d'État. A partir de 2002, l'organisation des concours nationaux réservés s'est ajoutée à celle des concours ordinaires. Un inspecteur général préside chaque jury, à l'exception de celui de conservateur. Dans ce dernier cas, un inspecteur général est vice-président, chargé du suivi et du contrôle administratif des activités du jury.

Il convient de rappeler ce que sont les tâches du responsable du jury. Celui-ci doit constituer le jury général et les commissions d'oral. Il s'agit non seulement de recruter le nombre de correcteurs et examinateurs nécessaires par rapport aux flux de candidats, mais aussi d'assurer l'équilibre des jurys, en termes de parité (équilibre hommes/femmes), et en termes d'origine professionnelle, d'expérience et de compétence. Les viviers ne sont pas extensibles. La charge se complique rapidement pour les concours à fort effectif. 
Il appartient au responsable de préparer les séances de travail du jury pour le choix des sujets, l'élaboration des barèmes de correction et d'évaluation. Il organise le suivi des corrections d'écrit, puis la réunion d'admissibilité ; les réunions des commissions d'oral, puis la réunion d'admission. A toutes ces étapes, il a le souci de garantir l'égalité de traitement des candidats.

Pour tous les jurys, sont collectés les éléments d'un rapport détaillé, mis en forme et diffusé à l'intention de l'administration, mais aussi des candidats et des centres de préparation.

En raison de ces tâches, la gestion d'un ou de plusieurs jurys pèse fortement sur le calendrier des inspecteurs généraux et conditionne une part de leur activité.

Le tableau ci-après décrit l'évolution des concours d'État de 1999 à 2003. Il indique les concours ouverts et pour chaque concours le nombre d'inscrits constaté.

\begin{tabular}{|c|c|c|c|c|c|}
\hline & 1999 & 2000 & 2001 & 2002 & 2003 \\
\hline Conservateurs & 2155 & 1419 & 1562 & 1272 & 1352 \\
\hline Conservateurs chartistes & 23 & 19 & 19 & 18 & 20 \\
\hline Bibliothécaires & - & 3628 & - & 2708 & - \\
\hline BAS & 865 & 756 & 656 & 471 & 645 \\
\hline Assistants & & & 4891 & 3714 & - \\
\hline Magasiniers en chef & 3681 & 2637 & 2324 & 1672 & 2027 \\
\hline Magasiniers spécialisés & - & 2522 & \multicolumn{3}{|c|}{ Recrutement direct } \\
\hline \multicolumn{6}{|l|}{ Concours Sapin } \\
\hline Bibliothécaires & & & & 44 & 33 \\
\hline Assistants & & & & 67 & - \\
\hline \multicolumn{6}{|l|}{ Examens professionnels } \\
\hline Bibliothécaires & & & 15 & - & - \\
\hline BA de classe exceptionnelle & 97 & & & & \\
\hline Assistants de classe exceptionnelle & & & 121 & 72 & 65 \\
\hline
\end{tabular}

Dans la période 1999-2002 le nombre des concours et des examens professionnels présidés par les inspecteurs généraux a fortement augmenté ( 5 en 1999, 6 en 2000, 7 en 2001, 9 en 2002), malgré le recrutement local des magasiniers spécialisés, prévu jusqu'en 2005. En 2003, 6 jurys seulement ont été présidés. Mais il y en aura à nouveau 9 en 2004.

Au cours des années 1999-2002, on a constaté dans les différents concours une baisse sensible et quasi générale du nombre d'inscrits. Mais, en 2003, les inscriptions au concours de BAS et de magasiniers en chef ont cru à nouveau. Pour les magasiniers en chef, l'offre d'emplois était cependant limitée (66 en 2003, contre 124 en 2002). Pour les BAS, le nombre de postes offerts était important (221, contre 174 en 2002 ) et il s'agissait de la dernière année où fonctionnaient les modalités exceptionnelles de recrutement.

En ce qui concerne les conservateurs, on peut penser que la forte augmentation des postes ouverts au concours de l'ENSSIB (36 postes en 2001, 47 en 2002, 52 en 2003...) augmente l'attractivité du concours et va attirer à nouveau des candidats plus nombreux. Au début de 2004, une remontée du nombre des candidats aux concours de recrutement d'assistants (4 348 inscrits) et de bibliothécaires (2 879 inscrits) était déjà constatée...

L'organisation des concours demeure donc une charge lourde pour l'Inspection et le contexte administratif demeure inchangé. Depuis le départ du conservateur responsable des concours, le département des concours de l'ENSSIB assure seulement la logistique. Les inspecteurs doivent traiter, sans l'appui de personnel de bibliothèque et avec un secrétariat limité à une personne, tout ce qui dans l'organisation d'un concours est intimement lié à la connaissance du métier et de l'environnement professionnel.

Si l'IGB considère que la responsabilité des concours découle de sa position au contact de deux ministères, de l'expérience des personnels et des établissements qu'apporte ses fonctions d'évaluation et de contrôle, elle continue à s'interroger sur les conditions dans lesquelles elle exerce ces responsabilités. 


\subsection{3. Études et programmes}

Les programmes donnés à l'Inspection en 2003 ont été présentés plus haut (1.1.).

L'Inspection a participé à une importante mission IGAC/IGB d'audit concernant la Bibliothèque nationale de France - qui annonce une redéfinition de ses programmes du côté du ministère de la Culture. Elle a également participé à une mission IGAENR/IGB sur un thème, le harcèlement moral au travail - dont il faut espérer qu'il ne se banalise pas.

En 2003, les missions de contrôle et d'évaluation concernant tant les bibliothèques publiques que les bibliothèques de l'enseignement supérieur ne comportaient pas, dans leur intitulé, d'étude thématique ou transversale.

Cependant, à travers l'ensemble des missions, des questions ont été examinées à plusieurs reprises et un certain nombre de thèmes d'intérêt général ont été abordés.

Comme en 2002, la moisson thématique est assez abondante. Le bilan des travaux, sous cet angle (2.2), a été organisé en trois parties :

- Les principaux dénominateurs des missions menées en 2003 dans le champ du contrôle permanent (bibliothèques publiques, bibliothèques universitaires).

- Les études approfondies faites sur une bibliothèque ou sur un groupe spécifique de bibliothèques, au cours de cette année.

- Deux thèmes traités dans différents types de bibliothèques (patrimoine imprimé, harcèlement moral).

La partie 2.3. "Les bibliothèques territoriales et la décentralisation" se distingue des précédentes. Il s'agit en effet d'une étude détaillée, qui développe une réflexion menée lors d'une conférence internationale. Si ce travail repose pour une part sur l'information apportée par les inspections, il s'appuie sur un large rassemblement de sources historiques et de statistiques. Une prospective est proposée.

\subsection{BILAN THEMATIQUE DES TRAVAUX}

\subsubsection{Principaux dénominateurs des missions d'inspection}

\subsubsection{Bibliothèques publiques}

En ce qui concerne les bibliothèques municipales, secteur d'inspection principal cette année, une bonne part des missions confiées à l'Inspection visaient à conforter et orienter des projets d'équipement.

Les situations constatées en 2003 sont diverses. Il peut s'agir de villes qui n'ont pas mis à jour leurs outils de lecture publique, de bibliothèques qu'il faut à la fois reloger et informatiser pour la première fois. Dans ces sites, on passera d'une bibliothèque du $19^{\text {ème }}$ siècle à une médiathèque.

Il s'agit bien plus souvent, dans la continuité d'une politique municipale de lecture publique, de réimplantations, de projets d'extension ou de restructurations, dont certaines ont été proposées par les inspecteurs.

Dans un cas, l'inspecteur a été chargé d'examiner les difficultés d'une bibliothèque municipale à vocation régionale (Reims) confrontée au succès considérable des nouveaux équipements ouverts au public, avec des problèmes de mise à niveau des effectifs, d'organisation du réseau de lecture publique. 
La position des inspecteurs a été parfois nouvelle. L'implication de L'IGB en amont de projets d'équipement pose des problèmes tant de charges de travail que déontologiques (l'Inspection ne saurait être à la fois juge et partie). Cela ne peut être qu'un mode d'action ponctuel, sur des projets particuliers (ce fut le cas pour certaines bibliothèques municipales à vocation régionale). A une autre étape, comment appuyer un projet de construction, alors qu'une étude est commandée en parallèle pour la collectivité ? Est-ce le bon moment pour que l'IGB intervienne ? L'IGB a-t-elle vocation à expertiser des études ? Dans un tel cas le rapport rendu est plus sommaire : moins descriptif, plus prospectif ; moins technique, plus politique ; moins explicatif, plus prescriptif.

De manière plus traditionnelle s'est posé çà et là le problème d'orienter un projet en ne disposant que de sources imprécises sur les moyens réels des communes. Dans ces cas, l'Inspection est portée au réalisme.

Un arrière-plan important et fréquent des inspections menées en 2003 était le passage à l'intercommunalité, le plus souvent une communauté d'agglomération.

Ce mouvement bouleverse la géographie institutionnelle de la lecture publique, avec des problèmes de natures différentes : définition des services de la bibliothèque communautaire, liens de la bibliothèque de la ville centre avec les bibliothèques de la communauté d'agglomération, rôle des bibliothèques départementales face à l'intercommunalité ? Dans une communauté d'agglomération telle que celle de Mont-de-Marsan, par exemple, 17 communes sur 18 ont moins de 10000 habitants.

Parmi les communes participant à la communauté d'agglomération Caen-la-Mer, créée le $1^{\mathrm{er}}$ janvier 2003, deux ont une bibliothèque importante : Caen et Hérouville-Saint-Clair. Tout a été transféré à la communauté d'agglomération, qui a la gestion des équipements culturels (bibliothèque centrale et bibliothèques de quartier). Un service interétablissements de coopération a été créé, dont la responsable est la directrice de la bibliothèque de Caen. La bibliothèque d'Hérouville a choisi le même SIGBD que Caen. Les problèmes qui apparaissent sont actuellement techniques : besoin d'une construction nouvelle à Caen, écrasée par le dépôt légal d'imprimeur, à l'étroit dans des locaux qui ont bien vieilli parce qu'ils avaient été bien conçus, harmonisation des collections et des services (Hérouville a fortement développé les nouveaux supports, ouvre le dimanche et a $28 \%$ d'inscrits).

La Rochelle offre le cas d'une intercommunalité complexe : la centrale relève de la communauté d'agglomération, les bibliothèques de quartier sont restées sous l'égide de la ville. Mais le tout a une direction unique, la volonté de développer la coopération intercommunale est forte.

Le transfert à Rennes-Métropole de la centrale doit avoir de même un impact sur le réseau rennais de lecture publique. Quel rôle sera-t-elle appelée à jouer auprès des bibliothèques des communes de l'agglomération ? Sera-t-elle une tête de réseau ou un centre de ressources pour l'ensemble de ces bibliothèques. Quelle sera alors sa place par rapport à la Bibliothèque départementale d'Ille-et-Vilaine (BDIV), traditionnellement centre de ressources et de services pour les bibliothèques des communes de moins de 10000 habitants ? Celle-ci bénéficie de la politique du conseil général pour la lecture publique et prépare la déconcentration de ses actions : mise en place d'ici 2006 de quatre antennes de pays devant jouer au niveau de ces territoires le même rôle que la BDIV pour le département. Quels seront les rôles respectifs de l'antenne du Pays de Rennes, de la BDIV et de la future BMVR des Champs-libres?

Lorsqu'elles regroupent des villes petites et moyennes, les communautés d'agglomération sont soucieuses d'impliquer autant que faire se peut le département et la médiathèque départementale dans le fonctionnement du futur établissement. Ce qui est envisagé peut apparaître comme une fusion des deux services. Techniquement ce type d'éventualité paraît difficile à retenir. Pour autant, les équipements communautaires regroupant de petites communes ne sauraient se passer des médiathèques départementales. Celles-ci doivent continuer à apporter leur appui aux communes de moins de 10000 habitants de la communauté d'agglomération, même si toutes les bibliothèques y sont mutualisées. 
Dans de nombreux départements, du fait du développement de l'intercommunalité, la limite de 10000 habitants assignée depuis une vingtaine d'années a perdu de sa pertinence. La réalité des pays conduit à reconsidérer la coopération locale. C'est avec l'ensemble des bibliothèques du pays que les antennes des BDP sont amenées à travailler.

Mais la coopération suppose que les médiathèques départementales soient associées à la conception des dispositifs communautaires, que les rôles soient précisés et répartis. Des réflexions sont nécessaires entre les deux types de collectivité, département et communauté d'agglomération et entre les bibliothèques concernées (bibliothèque départementale et médiathèque communautaire), pour mutualiser et optimiser les ressources.

Pour l'instant, il peut y avoir enchevêtrement, superposition, les décisions étant prises souvent en fonction de critères économiques et politiques, sans vision de ce à quoi on veut aboutir. L'inspection de la médiathèque intercommunale du Syndicat d'agglomération nouvelle (SAN) Ouest-Provence, établissement assez important (92 000 habitants) et appuyé sur une intercommunalité qui existe depuis les années 1970, a fait en revanche apparaître une sorte de modèle d'organisation bibliothéconomique.

C'est une bibliothèque intercommunale éprouvée et solide, puissante et structurée. Utilisant un schéma autre que le découpage habituel entre une centrale et des annexes, son organisation s'appuie sur des services communs efficaces et bien rodés (dont un circuit du livre centralisé) ainsi que sur une évaluation constante grâce à un excellent suivi statistique.

Le réseau est constitué des cinq bibliothèques du SAN, distantes et forcément disparates, mais cependant traitées sur un pied d'égalité. Il a su jouer la cohérence en terme d'aménagement des espaces, d'organisation thématique des collections multimédia, de politique documentaire, unitaire et maîtrisée. La grande force de l'établissement tient à un fonctionnement où la transversalité s'exerce sans nuire à la hiérarchie, avec un développement des services à distance, quasi indispensable dans un réseau multisite.

Alors que l'Inspection rencontre beaucoup d'intérêt pour les politiques documentaires, mais souvent des réflexions embryonnaires, la médiathèque du SAN offre le cas d'une politique formalisée et aboutie. L'activité de chaque acquéreur est encadrée par une "fiche domaine", dans laquelle il regroupe l'essentiel des informations utiles pour son secteur (dont un tableau de bord prévisionnel des achats, un bilan chiffré et commenté des acquisitions), et nourrie par les informations tirées de la base des acquisitions, un instrument complet de gestion des commandes.

Dans le même établissement, une étude récente permet d'alimenter la réflexion sur la baisse des statistiques des bibliothèques. Après diminution brutale des inscrits, une étude par sondage a été réalisée par la médiathèque en 2001, auprès des usagers n'ayant pas renouvelé leur inscription. Elle montre que si la médiathèque a confirmé une image positive auprès de ce public, la première cause de défection réside dans le fait que lecture et déplacement jusqu'à la bibliothèque prennent trop de temps. D'autres cas montrent que ce mouvement statistique est à analyser au vu de la population desservie intra muros et de son évolution propre.

Au delà des problèmes bibliothéconomiques, les inspecteurs ont eu à examiner à plusieurs reprises, au cours de leurs missions, des problèmes de moyens : bibliothèques récentes dont les crédits de fonctionnement passent brusquement au-dessous des moyennes nationales et problèmes de personnel. Même si cela semble paradoxal, on trouve des médiathèques neuves et fonctionnelles, sans directeur ou sans personnel professionnel à leur tête, alors qu'elles sont confrontées à de sérieux problèmes techniques (tarifs, horaires, politique documentaire, croissance exponentielle des prêts de nouveaux supports). Il n'est pas rare de constater, dans de beaux équipements, des relations tendues entre collectivité et directeur, concomitantes des restrictions budgétaires.

L'Inspection doit donc noter la relative fréquence des problèmes de personnel et d'encadrement, la fragilité des emplois professionnels dans les bibliothèques municipales (non remplacement de congés de longue durée, même s'il s'agit du directeur ; tendance à pourvoir les postes avec des personnels précaires, en utilisant des dispositifs d'emplois aidés (CES, EJ). 
Parallèlement nombre de collectivités ont fait des efforts pour amortir la fin des dispositions relatives aux emplois jeunes, en les intégrant sur des postes d'agent du patrimoine notamment.

Des changements d'équipes municipales peuvent mettre en difficulté certains directeurs; des équipements nouveaux réalisés par les équipes précédentes peuvent ne pas être "adoptés" par les nouvelles municipalités, qui leur disputeront les moyens de fonctionner. Cela n'est pas conforme aux principes qui régissent la permanence du service public et peut faire des fonctionnaires les otages de querelles politiques. Qui plus est l'investissement consenti peut tourner au gâchis d'argent public, quand les nouveaux bâtiments végètent au lieu d'attirer la population.

Mais on regrettera parfois le manque de sens politique de directeurs, qui se placent dans des situations compliquées et peuvent mettre les élus en difficulté. On a constaté dans certains lieux un discernement défaillant et le désir de placer les bibliothèques à l'abri de la vie municipale. En agissant ainsi on risque de les marginaliser et de légitimer à l'avance la faiblesse des moyens accordés.

Un autre aspect des problèmes de personnel, ce sont les tensions et conflits qui peuvent se développer entre des membres du personnel et le directeur de la bibliothèque, affaiblissant le plus souvent la position de ce directeur vis-à-vis de sa tutelle. L'Inspection examine périodiquement de telles situations - dans différents types de bibliothèques - et propose des solutions. En 2003, l'IGB a été amenée à trois reprises à examiner la question du harcèlement moral, dans différents champs d'inspection (cf. 2.2.3.2.).

\subsubsection{Bibliothèques d'enseignement supérieur}

Suivant un usage mis en place depuis plusieurs années, la majorité des inspections se déroulent avant le renouvellement des contrats et ont pour but d'éclairer la démarche contractuelle.

Les questions examinées à plusieurs reprises découlent donc pour une large part des thématiques des contrats. Ceux-ci font apparaître l'intégration des SCD dans l'université, sensible avec le développement du SIGB et mieux encore avec le développement d'un système d'information donnant accès à l'ensemble des ressources. C'est un outil de fédération de l'ensemble des unités documentaires de l'université, généralement éclatées. Dans les BU comme dans les BM d'importance, une certaine désaffection du public conduit à développer de nouveaux services (formation à l'Internet) et se traduit dans l'aménagement des locaux (espaces pour le travail de groupes).

Si les thèmes des contrats sont largement communs, dans les échantillons examinés en 2003, les résultats pouvaient être forts différents.

Il est vrai que les situations peuvent être elles-mêmes fort différentes. Selon les sites, les vastes programmes de restructuration et d'extension des bâtiments en cours se trouvent à des étapes diverses. Loin de la métropole, l'Université de Polynésie française doit en principe desservir des îles couvrant une surface grande comme l'Europe. Son SCD, relancé par une nouvelle directrice et maintenant mieux intégré dans l'université, rencontre des problèmes spécifiques : coût du fret pour les publications venant de métropole; pas de haut débit, d'où problème de connexion à l'Internet, absence de périodiques en ligne.

Ainsi, la politique de formation des étudiants a pu aboutir de manière exemplaire dans des SCD ou des SICD de tailles très différentes.

A Besançon, cette formation est intégrée dans les cursus. Douze personnes du SCD font de la formation, ce qui est très utile pour des nouveaux entrants issus de milieux souvent non favorisés. A La Rochelle, les actions en direction des étudiants de $1^{\text {ère }}$ année peuvent être institutionnalisées. L'université a mis en place une unité méthodologique dénommée PPP (projet personnel et professionnel), intégrée dans la maquette des enseignements pour l'ensemble des cursus littéraires. Pour les sciences de la terre, un mémoire et un oral sont pris en compte dans le DEUG. Les modules de méthodologie documentaire sont proposés avec le concours d'enseignants du secondaire et de 
l'université, de bibliothécaires de la BU, secondés par des tuteurs et une conseillère d'orientationpsychologue, détachée pour ce projet.

Dans un ensemble d'une toute autre taille, la BIU scientifique Jussieu, les modules de méthodologie du travail universitaire en DEUG ont connu une montée en puissance, des pages d'autoformation ont été réalisées sur le site Internet de la bibliothèque. Le noyau de moyens initiaux ayant fondu, la responsable a dû inventer d'autres solutions, en s'appuyant sur un groupe d'intervenants volontaires issus des sections d'enseignement.

Dans d'autres SCD (Polynésie, Rennes 1, Paris 7...), la formation des utilisateurs apparaît comme un point faible du bilan du contrat, les moyens en personnel ou la connexion avec les enseignants n'ayant pas été trouvés.

En ce qui concerne les politiques documentaires, les situations demeurent variables.

Le problème de prévision est rendu plus sensible et contraignant par la montée des périodiques électroniques, qui oblige à définir de grandes lignes directrices. Mais parallèlement, l'arrivée des périodiques électroniques emporte les crédits disponibles.

Dans les faits, nombre de politiques documentaires sont peu développées, pas véritablement formalisées. Il s'agit de la politique d'un SCD et non d'une politique de site universitaire. L'Inspection n'a pas rencontré récemment d'exemple de coopération entre bibliothèque universitaire et bibliothèque publique dans ce domaine.

En 2003, trois cas ont été examinés avec une particulière attention.

- A La Rochelle le SCD travaille avec méthode à passer d'une charte à un plan de développement des collections pour chacun des grands domaines disciplinaires couverts. Sans surprise, une enquête sur la politique documentaire pour la recherche a conclu au développement des abonnements aux bases de données et périodiques électroniques.

- Le SCD de Paris 7, qui doit constituer les collections de sa nouvelle bibliothèque centrale offre un modèle de travail sur des collections en lettres et sciences humaines. Suivant le modèle universitaire de Lausanne, les étapes ont été :

- analyse de l'existant (questionnaires aux BUFR),

- évaluation des collections du SCD en LSH, en 2002, anglais excepté faute de statistiques d'utilisation des fonds,

- mise en œuvre de politiques de développement des collections (PDC), précédée par la production d'un document généraliste : la charte des acquisitions, présentée à un conseil de documentation dédié. Tous les directeurs d'UFR étaient invités à ce conseil, présidé par le président de l'université en novembre 2002.

Les PDC par discipline s'appuient sur un relevé systématique des enseignements et secteurs de recherche, annexé. Ils quantifient le projet et fixent des objectifs pour les acquéreurs. C'est une base de discussion avec les enseignants-chercheurs. Trois PDC ont été réalisées, en histoire, en sociologie, puis en langues et littératures étrangères. Le SCD a travaillé avec des UFR réactives, en cherchant à combler des lacunes.

L'équipe d'acquéreurs est encore insuffisante, d'où le recrutement de vacataires, au niveau DEA. Ces vacataires, encadrés par des bibliothécaires, reçoivent une formation pour leur secteur d'acquisition et une formation technique pour la sélection, le traitement, la cotation.

Un conseil de la documentation est prévu pour présenter les PDC, les équipes d'acquéreurs, demander des correspondants (1 enseignement, 1 recherche). La méthode de travail s'efforce d'informer systématiquement les enseignants chercheurs (cadres diffusés, résultats présentés dans base PRG SUDOC), pour qu'ils s'associent à la constitution de l'outil documentaire.

Les collections prévues à l'ouverture de la nouvelle bibliothèque centrale sont de 200000 volumes en libre accès (150 000 en lettres et sciences humaines, 50000 en sciences), 1400 titres de périodiques (1 250 en sciences humaines, 150 en sciences).

L'Inspection a noté en outre les problèmes particuliers que pose l'accès aux périodiques électroniques dans des sites hospitaliers, car l'Assistance publique interdit la diffusion des adresses IP de son réseau (dossier examiné par le collectif 56711). Par ailleurs, on sait que les fournisseurs 
d'accès électroniques n'admettent pas le fonctionnement interuniversitaire, ce qui n'est pas une mince complication, lorsqu'une université (ici Paris 7) s'appuie pour son secteur scientifique sur une BIU.

- Le troisième cas correspond à une politique embryonnaire. Mais il est cité comme action pionnière. A l'occasion de l'inspection du SCD d'Aix-Marseille 1, un point a été fait sur la politique de site encouragée là par le ministère.

Les trois universités d'Aix-Marseille (près de 70000 étudiants au total) ont un partage des disciplines complexe, source de difficultés soulignée par le Comité national d'évaluation. Ainsi en est-il du morcellement des disciplines scientifiques, dont les enseignements sont présents à AixMarseille 1 sur le campus de Saint-Charles, mais aussi à Aix-Marseille 2 et 3.

Les universités sont à la recherche d'une politique commune, pour construire une cohérence, en générant des économies d'échelle. Entrent dans ce cadre, les regroupements de la physique-chimie et des sciences de la matière d'Aix-Marseille 1 et 3 sur les campus de Saint-Jérôme et de Château-Gombert. L'Université de Provence cherche aussi à rééquilibrer les flux de ses étudiants et à attirer une nouvelle population sur le campus scientifique Saint-Charles à Marseille.

Entre les trois SCD, la collaboration est à l'ordre du jour : coopération sur les nouvelles technologies, harmonisation des politiques documentaires, transferts de collections. Un comité d'harmonisation et de réflexion universitaire a été mis en place par les trois universités. Les effets sont encore lents à émerger.

En liaison avec les politiques documentaires, l'Inspection a été interrogée sur l'interruption des tableaux rendant compte de l'évolution du coût de la documentation universitaire. Dans ses rapports, de 1998 à 2001, l'IGB a fourni un instrument donnant les grandes tendances de l'évolution des prix moyens, par grandes disciplines, pour la documentation papier, française et étrangère, pour les cédéroms et les périodiques électroniques. Ces données étaient rassemblées par J.-P. Casseyre, avec l'appui d'une série de bibliothèques et sections. Ce travail était apprécié car il fournissait rapidement des éléments utiles à la préparation des budgets. Il a pu être critiqué comme un instrument appuyé sur un échantillon limité. L'IGB, considérant qu'elle assurait cette étude en quelque sorte par défaut, a interrogé en 2002 et 2003 la SDBD sur la reprise de ce travail statistique, soit par l'administration centrale, soit par une unité universitaire ${ }^{(5)}$. Ce dossier demeure à l'étude.

Au-delà des contrats, l'Inspection porte, depuis 1999 au moins, une attention particulière à l'évolution de l'organisation fonctionnelle des SCD et SICD.

Au cours des inspections de 2003, l'Inspection a noté un mouvement d'évolution, mais a aussi constaté que certains SCD ou SICD sectionnés n'ont pas encore trouvé l'équilibre entre les sections et les fonctions transversales.

Dans un SCD, au moment de l'inspection, les conservateurs avaient uniquement des responsabilités transversales (informatisation/développement des collections, fonds ancien et fonds spécialisés/projet sur le public). Les départements étaient communs à l'ensemble des implantations. Les actions de coopération émanaient de l'équipe centrale (directeur +2 adjoints au directeur). Cette organisation, radicalement différente des cadres habituels méritait évaluation par un nouveau directeur.

Dans d'autres cas, SCD ou SICD, le développement des fonctions transversales était en cours, avec des priorités très pratiques : accompagner les programmes de l'université, améliorer et homogénéiser l'offre de services au public...

A Rennes 1 , a été mise en place la gestion centralisée des abonnements de périodiques, papier et électroniques, pour l'ensemble de l'université. Ce service de trois personnes, placé auprès de la directrice, s'est doté d'outils analytiques et comptables et centralise les offres qu'il fait

\footnotetext{
(5) Sur le modèle de la Library and Information Statistics Unit de l'université britannique de Loughborough, qui compte parmi ses publications régulières Average prices of British and USA academic books et mène des études statistiques sur les bibliothèques publiques et les bibliothèques de l'enseignement supérieur.
} 
tester aux sections. Il s'appuie sur une commission scientifique interdisciplinaire. Composée de représentants des trois conseils de l'université et de professionnels des bibliothèques, la commission a une fonction de pilotage et de structure d'appel.

Dans une bibliothèque visitée, le SICD 2 de Grenoble, commun aux universités de Grenoble 2 et 3 , une réorganisation a été lancée à l'occasion de la rénovation et de la restructuration du site du SICD. C'est une départementalisation complète, à partir des anciennes sections.

Six départements ont été créés (public - département créé en priorité, collection, système d'information documentaire, administration, coopération documentaire). L'opération, liée à l'évolution de la place du SICD dans les universités, a été conçue comme un véritable projet de service : amélioration des interfaces avec le public, amélioration des acquisitions et des services. Un principe d'évaluation et d'adaptation a été posé au départ. Ainsi, dans le département collections, deux services ont dû être substitués aux pôles de responsabilité par disciplines. Le département public a été remodelé en quatre services (accueil, formation du public, communication des documents, action culturelle). L'accent a été également mis sur la coordination (réunions des responsables, cellule du planning coordonnant l'ensemble des obligations de service public du personnel).

Dans certains sites provinciaux visités, l'unité de référence demeurait la section, dans sa conception la plus traditionnelle, avec peu de structures transversales ou fédératives. Il en découlait suivant les lieux des inconvénients divers. Là, l'inspecteur constatait de fortes difficultés pour mutualiser des moyens pour les tâches communes entre sections partageant une même implantation, des politiques de service public fort différentes entre les sections, des problèmes de coordination des sites excentrés - pour lesquels l'université peut choisir une logique de site et non une logique de discipline. Ailleurs le problème se posait simplement en termes de visibilité et de lisibilité de la politique du SCD. Du moins une fonction évaluation était en cours de définition. Face à la dispersion des équipes, aux projets immobiliers à monter, la nomination d'un adjoint au directeur paraissait une solution possible.

De telles situations sont dues à l'histoire, à des facteurs contingents (notamment la multiplicité des sites, la répartition des personnels), mais aussi parfois à la difficulté à motiver une équipe sur le thème du changement de méthodes et de priorités - fût-il le vœu de l'université.

Cependant la dynamique documentaire a d'autant plus de chances de se mettre en place que l'université elle-même prêtera de l'attention au développement de sa documentation. Autrement dit, le goût pour le statu quo au sein d'un SCD n'aide pas au développement des politiques documentaires. Il est contradictoire avec la politique contractuelle, qui conduit à fixer des objectifs globalement au titre du SCD et au titre de ses sections et à faire évoluer les équilibres internes, notamment la répartition des personnels de catégorie $A$ et les responsabilités confiées aux cadres.

L'Inspection a déjà évoqué les changements d'organisation au sein des SCD dans les rapports 1999 (p. 25-26) et 2000 (p. 34). Elle constate qu'une évolution importante est en train de se produire. Lors de la première expansion universitaire des années soixante et soixante-dix, la majorité des bibliothèques universitaires françaises se sont découpées en sections, pour accompagner le découpage géographique des facultés. Seules des bibliothèques interuniversitaires parisiennes et des BU monosites ont échappé au sectionnement.

Les sections desservent chacune une discipline (lettres, droit, sciences, médecine...), avec généralement une équipe et des locaux distincts.

Dans un mouvement qui n'avait pas été prévu par le ministère, elles ont pris en charge, à partir des années 1970, non seulement le service au public, mais l'ensemble des fonctions internes (acquisition, catalogage...). Ce transfert de fonctions, opéré suivant l'initiative locale, n'a laissé au niveau central, auprès des directeurs des bibliothèques, que quelques services administratifs et techniques.

Ainsi s'est mise en place une organisation décentralisée, qui offre une grande variété dans le travail, mais comporte de réels inconvénients : éparpillement des compétences, accroissement des besoins en personnel, fractionnement des services sans économie d'échelle. C'est une organisation propre à la France. Nos BU diffèrent de leurs homologues étrangères, où prévaut un schéma 
encyclopédique et centralisé : une bibliothèque principale organisée en départements fonctionnels (constitution des ressources documentaires, services au public), qui appuie des annexes de service au public.

Les bibliothèques universitaires demeurent géographiquement découpées. Mais, depuis plusieurs années, l'organisation fonctionnelle de ces bibliothèques évolue, en liaison avec le développement des technologies et avec la contractualisation.

Sollicité de fournir un état des lieux, à l'occasion d'une session de formation continue sur les organisations transversales, formation pionnière organisée par le SCD de Metz ( $\mathrm{H}$. Colinmaire) et l'ENSSIB ${ }^{(6)}$, un des inspecteurs généraux (D. Pallier) a fait deux constats :

- Le premier est la généralisation du phénomène de recentralisation ou de développement des organisations transversales.

Le phénomène apparaissait dans la quasi totalité d'un échantillon couvrant $60 \%$ des SCD. Mais les formes étaient diverses :

- extension de la division des affaires générales, prévue statutairement auprès des directeurs. Son champ d'action passe de l'administration et de la gestion des ressources humaines à l'informatique, à la formation des personnels, à la coordination documentaire ;

- très fréquemment multiplication des missions personnelles, sous forme d'adjoints aux directeurs ou de chargés de missions auprès des directeurs. Un mode d'organisation apparaît où chaque conservateur se trouve responsable d'un secteur traditionnel (section, secteur documentaire) et d'une mission transversale ;

- plus rarement, reconstitution de services centraux pour des fonctions traditionnelles (acquisitions, catalogage), voire substitution de départements fonctionnels à des ensembles de sections, dans un projet ambitieux de réorganisation des services au public.

- Le second constat est l'absence de sources complètes et à jour permettant la réflexion sur ce mouvement et l'accompagnement éventuel de celui-ci. Un premier bilan a pu être fait en dépouillant les rapports d'inspection des cinq dernières années, qui semblent les seules sources disponibles sur l'évolution des organisations. Mais il est difficile d'extrapoler à partir de $60 \%$ des SCD, décrits à des dates diverses entre 1998 et 2002. L'évolution des organisations internes est rapide.

Jusqu'ici, une seule étude approfondie a été menée sur le sujet. Il s'agit du mémoire d'une élève de l'ENSSIB, rendu en $2003{ }^{(7)}$. Ce travail comporte trois parties : une analyse détaillée du contexte historique et statutaire, l'identification des forces centrifuges et des forces centripètes du niveau national au niveau de l'université, la présentation de modèles d'organisation fonctionnelle des SCD. La réflexion engagée a un grand intérêt. Des modèles d'organisation sont bien identifiés. Mais, le délai imparti à l'élaboration d'un mémoire ne permettait pas de lancer une enquête directe sous forme de questionnaire. Les informations utilisées sont celles données par les SCD disposant d'un site Internet - qui proposent rarement l'organigramme du service.

Pour avoir un tableau d'ensemble à jour une enquête directe paraît nécessaire.

L'Inspection générale des bibliothèques a signalé cette situation à la sous-direction des Bibliothèques et de la Documentation de la DES (SDBD) et proposé de prendre en charge une enquête dont le cadre serait élaboré avec la SDBD. Le projet a été soumis au directeur de cabinet du ministre de la Jeunesse, de l'Éducation et de la Recherche en septembre 2003. Celui-ci a donné son agrément. Le projet de questionnaire est en cours d'élaboration et sera soumis à un groupe d'experts avant envoi.

\footnotetext{
(6) Journées de formation "E6 - Mettre en œuvre une organisation transversale en SCD", organisées par le département de la formation continue de l'ENSSIB et le SCD de l'Université de Metz, 13-15 mai 2003.

(7) CLOT, Nathalie. Centralisation ou décentralisation ? Réflexions sur l'organisation fonctionnelle des SCD en France. Villeurbanne : ENSSIB, 2003, 100 f. Mémoire dirigé par Jean-Claude Annezer.
} 
Cette enquête ne portera que sur les SCD. Non que l'analyse de l'organisation des services interétablissements de coopération documentaire $(S I C D)$ et des bibliothèques interuniversitaires ne soit pas intéressante. Au contraire, elle peut faire apparaître des modes d'organisation plus libres et plus élaborés, pour plusieurs motifs. Leur cadre réglementaire est plus souple. Plusieurs de ces organismes bénéficient d'une unité géographique. Leur taille est souvent importante. En fait l'Inspection s'est penchée en 2002 et 2003 sur l'organisation de plusieurs SICD ${ }^{(8)}$. Ces organismes ne sont pas nombreux ( 8 à Paris, 8 hors île-de-France). Elle se propose de mettre à jour sa documentation par enquête directe auprès des SICD.

Une enquête sur l'organisation fonctionnelle des SCD devrait avoir plusieurs objectifs :

- Dresser un état des lieux complet et cohérent, qui fournisse des points de comparaison aux réflexions engagées localement. Il semble que plusieurs expériences soient déjà significatives et méritent d'être signalées. perspectives.

- Connaître l'avis des directeurs de bibliothèque sur ce mouvement, ses causes et ses

- Mieux identifier les champs de responsabilité confiés aux personnels d'encadrement. Cela paraît opportun à un moment où les procédures de notation et d'évaluation sont transformées.

- Identifier éventuellement des modes d'accompagnement. L'enquête pourrait être une source pour l'organisation de la formation continue dans les bibliothèques. Les personnels des bibliothèques ont la chance de bénéficier d'un appareil de formation solide (Département de la formation continue de l'ENSSIB, centres régionaux), piloté par la SDBD. II serait logique de prendre en compte prioritairement, dans les programmes de formation élaborés chaque année, les missions nouvelles confiées aux conservateurs, en phase avec les objectifs des universités.

\subsection{2. Études concernant des bibliothèques ou des groupes de bibliothèques}

\subsubsection{L'audit de la Bibliothèque nationale de France}

La BnF a connu, depuis sa création, de nombreuses missions d'évaluation ou d'inspection, la plupart liées à des périodes de turbulences, voire de crises, dont la plus emblématique a été la crise sociale de 1998. L'audit de 2003 a été engagé par la tutelle dans un tout autre contexte, celui d'une clarification des rapports entre l'État et un établissement public de premier plan.

La plupart des rapports antérieurs avaient recommandé l'élaboration d'un contrat entre la Bibliothèque nationale de France et le ministère de la Culture, garantissant l'autonomie de la personne morale dans le cadre des principes de la tutelle et des priorités nationales que celle-ci édicte. La note adressée le 24 décembre 2002 par le ministre aux présidents et directeurs d'un certain nombre d'établissements publics rappelle ces principes et précise de manière particulièrement nette les grandes orientations fixées pour les années 2003 et suivantes.

La conduite d'un audit de la BnF a été prescrite par le directeur du cabinet du ministre de la Culture dans la perspective de la conclusion d'un tel contrat. Cet exercice était conçu comme le moyen, d'une part d'apprécier "la façon dont l'établissement remplit ses missions", d'autre part "de valoriser et de développer la fonction de contrôle de gestion dans la perspective de la nouvelle loi organique relative aux lois de finances".

Le 6 novembre 2002 a été retenu le principe d'un audit effectué par l'Inspection générale de l'administration des affaires culturelles et par l'Inspection générale de bibliothèques, avec le concours soit de l'Inspection générale des finances, soit d'un cabinet d'audit privé.

La mission a été officialisée le 26 février 2003 en retenant la formule d'un cabinet d'audit dont l'intervention aux côtés des inspecteurs devait permettre la remise d'un rapport "au plus tard le $1^{\text {er }}$ juin 2003" et celle d'une note préalable définissant "les contours plus précis que l'audit et le futur contrat d'objectifs et de moyens pourraient adopter", le $1^{\text {er }}$ avril.

\footnotetext{
(8) Rapport 2002, p. 68-81, "Les bibliothèques interuniversitaires".
} 
Cette hypothèse impliquait que l'intervenant extérieur fût en état de commencer ses travaux en même temps que les inspecteurs: or le cabinet d'audit spécialisé, qui devait être désigné dans un délai lui permettant d'intervenir de manière cohérente et en concertation avec la mission, n'a pu commencer ses travaux que le $1^{\text {er }}$ juillet, le projet de cahier des charges, préparé par les services de la direction de l'administration générale et examiné par la mission d'inspection dès le 28 février, n'ayant pu être validé par les administrations centrales qu'à la fin du mois de juin.

Dans ces conditions, les inspecteurs ont souhaité élaborer, plutôt qu'une "note méthodologique détaillée", un pré-rapport laissant place pour des investigations complémentaires et permettant de définir et préciser les termes du cahier des charges destiné au cabinet d'audit. $\mathrm{Ce}$ pré-rapport a été remis au cabinet du ministre pour le $1^{\mathrm{er}}$ avril.

Dans un souci d'exhaustivité, les inspecteurs ont entrepris, en juillet, un second rapport pour compléter et finaliser leurs conclusions. En tenant compte de la "commande" passée au consultant, ce rapport tend à préciser les capacités de l'établissement à surmonter ses faiblesses et à exploiter au mieux ses atouts pour un meilleur accomplissement de ses missions.

Parallèlement, la mission a suivi l'avancement des travaux du consultant dans ses investigations, au cours de réunions de définition et de suivi de travaux qui ont eu lieu les 2 et 29 juillet. L'ensemble des rapports a été rendu au directeur du cabinet du ministre pour le 31 juillet.

Le document final rassemble, sous la dénomination commune Rapport d'audit de la BnF en vue de la préparation d'un contrat d'objectifs et de moyens, quatre tomes :

- Le tome I (Rapport général d'audit), constitue la synthèse de l'ensemble des travaux.

Son volume 1 (Pré-rapport) expose les principales lignes de raisonnement, constats, diagnostics et propositions pouvant être formulés en réponse aux orientations prescrites par la lettre de mission. Par ailleurs, pour des raisons tenant aux circonstances, la mission ne s'était, à ce stade, pas rendue sur le site de Richelieu pour prendre plus précisément connaissance des problèmes qui affectent les conditions de conservation des collections spécialisées. Enfin, à la date d'achèvement de ce pré-rapport, leurs auteurs n'étaient pas en mesure d'y intégrer les informations contenues dans le projet de réponse, établi par la BnF, au relevé de constatations provisoires de la Cour des comptes.

Le volume 2 (Patrimoine et transmission) est consacré aux conditions de mise en œuvre des missions réglementaires dont la BnF a la charge.

Le volume 3 présente les principaux éléments d'analyse organisationnelle et financière, résultat de la "commande" passée au consultant, retenu pour apporter un appui technique aux inspecteurs - avec l'espoir d'un "rendu" quantitatif et "expert" sous l'angle économique (terme qui inclut les analyses intéressant l'organisation et les effectifs) et financier.

- Les tomes II, III et IV comportent des documents complémentaires et des annexes.

- L’Audit financier - Analyse détaillée, qui décrit les éléments caractéristiques de la structure financière de l'établissement, la mesure des enjeux, les risques et simulations pouvant servir à l'appréciation des termes économiques et budgétaires d'un futur contrat : conformément au cahier des charges imposé au consultant, une simulation a en particulier permis d'expliciter, sous l'angle budgétaire, les conditions de fonctionnement de l'établissement suivant différentes hypothèses d'évolution très contrainte de la subvention de fonctionnement.

- L'Audit organisationnel - Analyse complémentaire, qui apporte, en complément des réflexions relatives à l'exercice des missions, un éclairage particulier sur les choix d'organisation et la recherche de l'efficience en termes de moyens, comme sur les principaux processus mis en œuvre par l'établissement.

- Les Annexes documentaires, qui rassemblent les principaux documents de référence rappelés dans les différents volumes. 
Les réflexions ont porté tant sur la mise en œuvre des missions de la BnF que sur les modalités organisationnelles et financières de son fonctionnement, suivant en cela les préoccupations exprimées dans la lettre de mission : établir une synthèse des évaluations déjà réalisées, une analyse des capacités de l'établissement à assurer les missions réglementaires qui lui sont imparties (notamment celle de l'animation d'un réseau de coopération documentaire), une réflexion sur ses modalités d'organisation, sur la gestion de ses ressources humaines et sur l'hypothèse d'un transfert à son profit des emplois que l'État lui affecte.

Bien entendu, l'analyse de ces différentes questions a été conduite avec la préoccupation "critique" des conditions d'une meilleure productivité possible. Elle a été assortie d'une analyse financière permettant d'étudier les marges de manœuvre qui pourraient être dégagées par l'établissement sur son budget de fonctionnement.

\section{$>$ Les constats}

Le contexte de rigueur budgétaire impose l'affirmation de priorités, la recherche plus attentive de rentabilisation des moyens, la mise en place d'une architecture de contrôle de gestion au profit partagé de l'établissement et de la tutelle. Mais ces impératifs ne peuvent décemment pas constituer l'apanage d'une gestion publique appliquée aux seuls "temps difficiles".

Par ailleurs, la tutelle ne s'exerce sans doute pas toujours à un niveau souhaitable. La mission croit devoir insister sur ce point qui intéresse la plupart des établissements publics, mais s'applique, dans le cas étudié, à une institution dotée de capacités qui déséquilibrent son rapport avec les services de l'État. Loin de lui être profitable à la veille de la négociation d'un contrat d'objectifs et de moyens, cette situation risquerait d'enfermer l'opérateur culturel et les représentants de l'État dans un débat où leur nécessaire rencontre sur des bases de connaissance et de réflexion partagées serait par trop absente.

Sous réserve d'une négociation dont les termes et les conclusions pourraient à la fois servir un intérêt général qui les dépasse et aboutir à un équilibre "gagnant - gagnant", la définition clarifiée des rapports entre l'État et l'établissement dans une ambition dynamique quant aux missions et capacités de gestion n'exclut évidemment pas un cadrage "volontariste" quant à l'évolution de la dépense. Dans le cadre de finalités culturelles précisées par ailleurs, d'un souci contributif à la mobilisation des "forces vives" de l'institution, il importe en effet de se demander quelle ambition "motrice" le ministère consent à l'institution. Tel a été le souci majeur (et commun à ses différentes composantes) des auditeurs.

A cet effet, il convenait d'identifier le cadre de l'action de l'établissement, celui de l'allocation de la ressource qui légitime le rôle de la tutelle en disposant d'indicateurs d'évaluation en rapport avec les objectifs à atteindre et les moyens à mettre en œuvre et assurer postérieurement au contrat - un suivi régulier de sa mise en œuvre grâce à une remontée régulière à la tutelle d'informations consolidées de la part de l'établissement.

La mission a envisagé le contrat objectifs-moyens comme devant être tout à la fois :

- le prolongement de l'actuel projet d'établissement qui recouvre la quasi totalité des responsabilités de l'établissement ;

- l'approfondissement d'une démarche collective de réflexion sur les moyens (qui ne sont pas exclusivement financiers) d'aboutir. Un tel résultat offrirait aux instances décisionnelles et au conseil d'administration des conditions optimales pour faire progresser l'établissement ;

- une nouvelle étape du développement de l'institution par l'affirmation de priorités ministérielles définies dans le cadre des missions réglementaires ;

- la présentation d'une articulation entre les efforts consentis par la collectivité nationale (moyens et capacité de gestion renforcée) et le contenu des engagements de l'établissement (amélioration du service public, optimisation des résultats) ;

- la mise en place d'outils de suivi partagés par la tutelle et l'établissement notamment ceux devant résulter d'une analyse préalable des résultats à atteindre ; 
- l'exercice d'un contrôle favorisant la meilleure préparation possible d'un prochain projet de contrat objectifs-moyens.

Au delà des objectifs susceptibles d'être inscrits dans un contrat, la mission a pu relever que les modes d'accomplissement des missions ne se formulaient pas de manière totalement comparable aux différent niveaux de la hiérarchie et qu'il convenait d'envisager le futur contrat comme un vecteur de plus grande unité sur la compréhension et la mise en application des orientations de l'établissement.

C'est pourquoi il a paru utile et possible à la mission de faire des propositions qui rationalisent l'organisation autour de ces grandes missions réglementaires, notamment en évacuant les "scories" organisationnelles inutiles ou dépassées.

La bonne préparation de l'institution pour cette démarche est incontestable et doit crédibiliser son action au regard d'un positionnement clair et engagé de l'autorité de tutelle. En effet, le contrat en vue duquel cette étude a été menée ne répondra aux intérêts des parties qu'à la condition que chacune soit motivée pour l'aboutissement dudit contrat et pour son exécution. Telle est bien la perspective garantie par la note que le ministre de la Culture a adressée aux présidents et directeurs d'établissement le 24 décembre dernier.

Comme l'exprime le rapport au fil de ses analyses, l'établissement qui a fait la preuve de son aptitude à la gouvernance doit voir ses efforts de modernisation davantage relayés au niveau des services. Les compétences scientifiques et techniques de l'encadrement ne sont pas en cause, non plus que son dévouement. D'ailleurs, conformément à l'attente de beaucoup d'entre eux et à une tradition illustrée lors de la mise en place du nouvel établissement, leur mobilisation est sollicitée pour permettre la réussite d'un contrat futur.

Naturellement, la réussite d'un contrat d'objectifs implique une transformation non moins importante des méthodes d'exercice de la tutelle ${ }^{(9)}$. Celle-ci doit, tout d'abord, adhérer à une perspective dépassant les préoccupations trop gestionnaires. Elle doit ensuite la codéfinir puis exploiter des outils de contrôle de gestion.

Ces deux remarques - l'engagement des cadres et l'adhésion de la tutelle - paraissent fondamentales à la mission, alors qu'elles ne figurent évidemment pas dans le projet d'établissement, mais qu'elles en conditionnent largement la réussite.

Enfin, le rôle du personnel d'exécution dans le bon accomplissement du contrat ne doit pas être sous-estimé.

On rappellera à cet égard que les personnels sont très attachés à la conclusion d'un tel document. Ainsi, lors de la "crise" sociale de 1998, les groupes de travail dont les analyses avaient enrichi le rapport Poirot avaient revendiqué l'élaboration d'un projet de ce type qui participât du souci de visibilité sur l'avenir de l'institution, de l'aspiration à une unité sociale plus affirmée et d'une appartenance reconnue à la sphère d'influence du ministère de la Culture. Il ne fait pas de doute que l'impression d'atermoiements dans la mise en œuvre d'un projet pour l'établissement serait mal ressentie et que la crédibilité de la direction de la bibliothèque pourrait en être ternie.

Ces considérations s'ajoutent à celles relatives au contenu des engagements pour considérer que - socialement aussi - le futur contrat devrait se situer dans la continuité de l'actuel projet d'établissement, tout en privilégiant le développement ou la prééminence de certaines priorités. Aux yeux des rapporteurs, les modalités de gestion des ressources humaines doivent être l'un de ces axes privilégiés. Il suppose aussi la capacité des parties à expliquer que les hypothèses de transfert ne portent en rien atteinte aux garanties que le statut permet de développer.

La concertation doit donc contribuer à "donner du sens" à un tel projet.

\footnotetext{
(9) Le cas de la BnF ne paraît nullement exceptionnel par rapport aux autres établissements, s'agissant de la mise à niveau et de la modernisation des méthodes d'exécution de la tutelle. Il y a là un "chantier" de modernisation important pour l'ensemble du ministère.
} 


\section{$>$ Les préconisations}

La thématique, Patrimoine et transmission, rassemble les questions et les préconisations que la mission des deux inspections générales a cru utile de traiter en approfondissement de son pré-rapport, en articulation avec l'audit économique et financier. En schématisant la réflexion, on pourrait dire que l'organisation thématique s'applique aux activités qui conditionnent l'existence et le développement d'une institution ayant pour vocation l'enrichissement et la conservation du patrimoine dont elle est détentrice. Mais la communication et la transmission de ce patrimoine sont très largement tributaires des mesures de rationalisation et de valorisation, qui, à la mesure des capacités de l'institution, seraient à même d'optimiser ses moyens.

Elle est aussi inspirée par le souci de répondre immédiatement et de manière concrète à l'attente de l'établissement, qui s'est montré très "réactif" aux interventions des inspecteurs et en particulier au contenu de leur pré-rapport ${ }^{(10)}$. Plus fondamentalement, ce second rapport est conçu, dans le prolongement de ce dernier document, c'est-à-dire avec l'ambition de contribuer à éclairer les choix - et le positionnement - des parties à l'éventuel contrat à venir, tant dans sa mise en œuvre que dans la dynamique de son suivi pluriannuel.

Aussi, le volume II du Rapport général permet-il d'entrer dans la réflexion relative au niveau de service à atteindre par mission. Il recommande également l'actualisation de certains contenus des missions réglementaires, notamment en raison des avancées techniques susceptibles de permettre à la $\mathrm{BnF}$ de jouer pleinement son rôle de tête d'un réseau documentaire pour la recherche et de répartition des compétences à l'échelle nationale.

De ce point de vue, l'étude sur la mise en œuvre des missions est confortée par les travaux des consultants qui sont plus précisément orientés vers l'analyse organisationnelle et l'adaptation des moyens à cette organisation. Ainsi une totale convergence apparaît-elle, par exemple, dans les préconisations tendant au maintien de la structuration par département spécialisé ou thématique, à la condition essentielle d'une clarification des compétences et des responsabilités; dans celles qui appellent une hiérarchisation des priorités en liaison avec la politique de réseau; dans celles qui invoquent une indispensable rationalisation des fonctions et des structures thématiques, dans les considérations relatives à la politique des publics et à l'accueil.

Les auditeurs insistent d'ailleurs sur le fait que ces seules préconisations - qui sont de nature à améliorer, concrètement et à court terme, la productivité des moyens existants - militent en faveur de la négociation d'un contrat dans un débat qui - sur ces points importants - pourrait sans doute être assez largement déconnecté de celui qui porterait sur le niveau d'accroissement des moyens.

En ce qui concerne l'appréciation des conditions financières de fonctionnement de l'établissement, les analyses des différents auditeurs sont identiques : les conclusions du prérapport sont illustrées et détaillées par les analyses du consultant privé, qu'il s'agisse par exemple de la structure - extrêmement rigide - des dépenses et de leur couverture par la subvention d'État, (on notera en particulier l'analyse comparative faite par le consultant des hypothèses d'augmentation de la subvention au regard des coûts salariaux qui résulteraient d'un transfert d'emplois), qu'il s'agisse des enjeux et des risques (projets de développement et investissement en cours ou prévus ; obligations légales conduisant à la dé-précarisation des agents ; conditions d'amortissement des installations techniques liées au bâtiment et aux systèmes d'information), qu'il s'agisse de l'appréciation des marges de manœuvre (politique d'achat, réorientation de certaines modalités de la gestion des équipements, redéfinition de certaines missions) ou qu'il s'agisse encore du développement des recettes propres (celles liées à la perspective de développement de la vente de produits dérivés et surtout de services en ligne).

\footnotetext{
(10) Les constatations, diagnostics et préconisations du pré-rapport de mars 2003 ont donné lieu, dans les directions et dans la majorité des départements, à des réunions de réflexion comme à l'élaboration de documents d'orientation sur des sujets prioritaires développés dans cette étude. Ils ont, en outre, conduit à l'organisation de deux séminaires au plus haut niveau de l'établissement.
} 
Cohérent, l'audit paraît également opérationnel parce que utilisable dans le court et le moyen terme.

En effet, certaines préconisations figurant dans le rapport général et intéressant les missions sont déjà engagées par l'établissement. Ainsi en est-il de la réflexion sur la réforme du dépôt légal, de l'achèvement du travail sur la charte documentaire, de la définition d'une véritable politique de conservation, de la réflexion sur la vocation réelle du Haut de Jardin.

D'autres, intéressant l'organisation, seraient susceptibles de trouver un écho rapide. Ainsi en serait-il du renforcement de l'efficacité de la DCO (11) par la mise en place de structures transversales et la mutualisation de certaines fonctions (service public ; catalogage). Parallèlement, la reconnaissance d'un rôle scientifique à la DSR ${ }^{12)}$ (mission scientifique ; responsabilité du réseau et de la bibliothèque numérique; élaboration de la politique de conservation) implique certainement les délais nécessaires au mûrissement de la réflexion interne, mais n'impose pas une perspective pluriannuelle pour la prise de décision.

En ce qui concerne la gestion des personnels, l'établissement paraît déterminé à poursuivre la réforme engagée des modes de gestion et à anticiper les conditions de prise en charge des emplois d'État qui seraient éventuellement transférés.

Dans le moyen terme peut être envisagée la relance d'une politique de réseau lisible et pertinente, ancrée dans une action territoriale. Une politique des publics clairement définie (illustrée entre autres par l'amélioration de l'accueil à différents niveaux sur les deux sites parisiens) peut également trouver à se préciser au cours des toutes prochaines années. II en va de même pour l'action internationale à renforcer, la vocation culturelle à confirmer et à approfondir, une politique commerciale à définir et à mettre en œuvre avec des outils et des compétences adaptés, une promotion de l'institution dans son environnement physique et intellectuel, une culture de l'évaluation à étendre.

Pour reprendre les termes du consultant, les analyses présentées et développées dans les documents joints "comportent toutes les réflexions utiles à la négociation, à la conclusion et à la mise en œuvre d'un contrat d'objectifs et de moyens".

Sans conduire à l'expression d'un vœu quelconque en ce sens, les observations - et les responsabilités - exercées à l'occasion de cet audit autorisent à insister sur l'intérêt d'une démarche réciproque, cohérente et articulée des services exerçant la tutelle avec celle de l'établissement public.

La BnF est forte de nombreux pôles d'excellence dans divers domaines : conservation, nouvelles technologies, histoire du livre, traitement des documents spécialisés, bibliographie. Ses capacités de gestion sont certaines ; les facteurs d'évolution récente vers la sincérité des prévisions budgétaires et de présentation des résultats en atteste. Elle paraît à même de tirer profit des réflexions contenues dans les documents d'audit.

La LOLF' implique une clarification des rapports de l'opérateur avec les tutelles et un contrôle de gestion adossé à une appréciation réaliste des coûts.

La responsabilité des ministères - et d'abord de celui qui pilote ces réformes - invite sans doute à une réflexion conduite parallèlement sur les considérations de pur affichage qui pourraient - à l'occasion de la négociation attendue - être présentées comme pouvant faire obstacle à ces ambitions.

Ainsi, la Bibliothèque nationale de France, premier établissement relevant de la tutelle du ministre de la Culture, apparaît aujourd'hui comme une institution qui a surmonté le défi du déménagement et de l'ouverture des nouveaux services sur le site de Tolbiac. De nouveaux enjeux se présentent aujourd'hui :

\footnotetext{
(11) Direction des Collections

(12) Direction des Services et Réseaux
} 
- la poursuite et la modernisation des missions traditionnelles (dépôt légal, catalogage, conservation);

- l'élargissement des missions nouvelles (ouverture aux publics, consolidation du réseau) ;

- le maintien à niveau des outils technologiques ;

- l'optimisation de la gestion.

Or le contexte de rigueur budgétaire impose l'affirmation de priorités, la recherche plus attentive de rentabilisation des moyens, la mise en place d'une architecture de contrôle de gestion au profit partagé de l'établissement et de la tutelle. La mission a cru devoir insister sur le dernier point, qui certes intéresse la plupart des établissements publics, mais s'applique dans ce cas particulier à une institution dotée désormais de toutes les capacités d'un opérateur culturel national et international.

C'est à une institution "en état de marche", capable de faire face aux enjeux de l'avenir, qu'ont été présentées les conclusions de l'audit, lors d'une réunion présidée le 15 septembre par le directeur de l'Administration générale du ministère de la Culture, en présence des représentants de la direction du Livre et de la Lecture. L'ensemble de l'audit a été validé lors d'une dernière réunion réunissant les mêmes intervenants, le 20 octobre.

Dès le 28 octobre, une première réunion d'élaboration du futur contrat réunissait la direction de la BnF et la DAG. Comme le présuppose la perspective de la conclusion d'un contrat d'objectifs et de moyens entre la personne morale et sa tutelle, il y a là, à la fois, le constat d'un remarquable mûrissement de l'institution (cinq ans d'existence est une durée courte dans la mise en fonctionnement d'une bibliothèque - et plus encore pour un équipement de ce type) et la perception d'un "moment" crucial dans l'histoire de la jeune Bibliothèque nationale de France : celui d'une nouvelle étape à engager dans la réflexion sur les enjeux par rapport aux forces et faiblesses de l'institution, dans la formalisation des objectifs et dans la définition des méthodes et moyens pour y parvenir.

\subsubsection{Les bibliothèques universitaires d'île-de-France}

L'Inspection acquiert depuis plusieurs années une meilleure connaissance des bibliothèques de l'enseignement supérieur en île-de-France.

Par le passé, le contrôle des bibliothèques universitaires de cette zone géographique, plus proches de l'administration centrale, a été épisodique. Depuis la reconstitution de l'effectif de l'Inspection en 1996, l'IGB a reçu chaque année des programmes comprenant des bibliothèques universitaires des académies de Paris, Versailles et Créteil. Ces programmes ont porté non seulement sur les SCD d'universités mais également sur les bibliothèques interuniversitaires. Souvent celles-ci n'avaient plus été inspectées depuis très longtemps.

Au cours des dernières années, tous les SCD des universités d'île-de-France ont fait l'objet d'un contrôle, à l'exception du SCD de Paris 8 . Les $3 / 4$ des BIU ont été inspectées : orientales,

- 1996 : Bibliothèque interuniversitaire de médecine (BIUM) et Bibliothèque des langues (BDIC),

- 1997 : Bibliothèque Cujas et Bibliothèque de documentation internationale contemporaine

- 1998 : Bibliothèque Sainte-Geneviève (BSG),

- 2003 : Bibliothèque interuniversitaire scientifique Jussieu (BIUSJ).

Dans un mouvement parallèle au contrôle des SCD d'universités, l'IGB a été chargée d'inspecter les bibliothèques de plusieurs grands établissements parisiens (Observatoire, École normale supérieure de la rue d'Ulm et École des chartes en 1997 ; Collège de France en 1998, Institut national de recherche pédagogique en 1999, Conservatoire national des arts et métiers en 2001).

Au delà du contrôle, les responsabilités assumées par D. Renoult ont conduit l'Inspection a être constamment informée des actions en cours dans les trois académies. 
Les rapports annuels de l'Inspection ont rendu compte des travaux du comité stratégique des bibliothèques universitaires d'île-de-France ${ }^{(13)}$. De même, la participation de D. Oppetit et de $D$. Renoult au comité d'évaluation des pôles universitaires d'île-de-France, mis en place par la Région, donne à l'IGB une meilleure connaissance de la vie étudiante. On a donné ci-après le point du programme U3M.

En complément, il a paru utile d'évoquer brièvement deux bibliothèques en forte évolution dans le cadre d'U3M, qui ont été inspectées cette année.

\section{$>$ Mission U3M Île-de-France en 2003 - Organisation}

L'année 2003 a été marquée par un tournant dans les modalités de suivi des projets de réhabilitation ou de construction de bibliothèques en Île de France. En effet à la suite du départ de H. Haugades, D. Renoult a pris la direction de la mission U3M, soit la responsabilité du suivi de l'ensemble des projets immobiliers inscrits au contrat de plan État/Région (CPER) et plus seulement du seul secteur des bibliothèques. Par ailleurs, V. Chabbert, ingénieur d'études a obtenu sa mutation pour le Comité national d'évaluation des établissements publics à caractère scientifique, technique et professionnel (CNE). Simultanément les projets de bibliothèque directement gérés par la Chancellerie des universités prenaient leur autonomie juridique et budgétaire (cf. infra).

Tandis que les années 2001 et 2002 avaient été surtout consacrées à préciser une stratégie et des orientations pour une région qui regroupe un grand nombre de bibliothèques qui au plan national conserve plus de $34 \%$ des monographies et $57 \%$ des collections de périodiques, l'année 2003 s'est caractérisée par la livraison de quelques opérations (Paris XII - Créteil, Fondation nationale des Sciences Politiques) et surtout par l'intensification des phases d'études.

\section{$>$ Avancement des projets du CPER 2000 - 2006/ U3M}

Ainsi ont pu être approuvés les avant-projets définitifs correspondant à la construction des quatre premiers bâtiments prévus pour l'université Paris 7 sur la ZAC Rive Gauche et notamment la réhabilitation de l'immeuble des Grands Moulins de Paris qui accueillera la future bibliothèque universitaire. Parallèlement étaient signées les conventions par lesquelles l'État confie la maîtrise d'ouvrage de $21000 \mathrm{~m}^{2}$ de surfaces d'enseignement et de recherche (Paris 7) au département de Paris, et à la région île-de-France la maîtrise d'ouvrage du pôle "Langues et civilisations" qui inclut la bibliothèque universitaire des langues et civilisations (BULAC). En 2003 également ont été finalisées les études d'architecture du projet Sainte-Barbe (APS et APD) tandis que se poursuivaient les travaux sur le site de Guyancourt pour l'université de Versailles-Saint-Quentin.

L'évaluation à mi-parcours du contrat de plan a été repoussée à l'année 2004 par la préfecture de Région. Cependant, en partenariat avec la Région île-de-France les travaux préparatoires à l'évaluation du CPER ont commencé avec la mise au point d'études descriptives détaillées sur l'ensemble des aspects de l'enseignement supérieur : situation en 1999 et en 2003 des effectifs étudiants, du patrimoine immobilier, du logement étudiant, des bibliothèques.

\section{$>$ Étude sur les publics des bibliothèques universitaires de l'académie de Paris}

Dans le cadre de la préparation des projets de bibliothèque de l'académie de Paris, une première enquête par sondage a été lancée auprès des étudiants en lettres et sciences humaines inscrits dans les universités parisiennes. Portant sur un échantillon de 1500 personnes, cette enquête qui a été effectuée à la fin du dernier trimestre pour partie à la sortie des bibliothèques (1 000 personnes) et pour partie à la sortie des cours a pour objectif de mieux connaître les comportements des étudiants en complétant les données statistiques des bibliothèques, et de mesurer les phénomènes de plurifréquentation en identifiant les comportements disciplinaires. Le questionnaire a été élaboré en concertation avec les directeurs de bibliothèques concernées. En cours d'exploitation, les résultats devraient être publiés à la fin du premier trimestre 2004.

(13) "Le comité stratégique des bibliothèques universitaires en île-de-France", Rapport annuel 2001, p. 64-66 ; "Les bibliothèques interuniversitaires", Rapport annuel 2002, p. 68-81. 


\section{$>$ Évolution du statut des bibliothèques interuniversitaires d'île-de-France}

Sur le plan statutaire ont été prises deux décisions importantes relatives d'une part au statut de Sainte-Barbe et d'autre part à celui de la BULAC. Après concertation avec les présidents d'université, a été préparée la convention constitutive de la bibliothèque Sainte-Barbe, service interétablissements de coopération documentaire rattaché à l'université de Paris III-Sorbonne nouvelle. Les universités de Paris I, Paris II et Paris IV sont contractantes à cette nouvelle bibliothèque interuniversitaire. La convention a été adoptée par les universités en décembre 2003. La modification consécutive du décret statutaire devrait intervenir, le CNESER entendu, au cours du premier semestre 2004.

Plus original, le statut adopté pour la BULAC utilise pour la première fois pour une bibliothèque la formule de Groupement d'Intérêt Public prévue par le décret 91-321 (T I, art. 2). Cette formule revêt en effet l'avantage de donner une structure juridique et budgétaire solide à la préfiguration d'un projet interuniversitaire dont les partenaires sont nombreux et variés, : 4 universités (Paris I, Paris III, Paris IV, Paris 7), 4 grands établissements (l'Institut national des langues et civilisations orientales, l'École Française d'Extrême-Orient, l'École Pratique des Hautes Études, l'École des Hautes Études en Sciences Sociales) et un établissement public à caractère scientifique et technique (le CNRS). Administrée par un conseil d'administration de 13 membres, la BULAC a été officiellement créée en décembre 2003. Un conseil scientifique de 15 à 20 membres devrait être installé en 2004.

\section{$>$ L'évolution du campus Jussieu. Le nouveau site Paris Rive Gauche}

La Bibliothèque interuniversitaire scientifique Jussieu (BIUSJ) est une des principales bibliothèques universitaires françaises, un instrument documentaire puissant, aux collections importantes. Suivant les statistiques 2001, la BIUSJ se trouvait alors au troisième rang national pour le nombre d'emplois, au sixième pour les recettes budgétaires ; en termes de services, elle se situait au quatrième rang national pour le nombre d'usagers, au septième pour le nombre de prêts.

Parmi les BIU, sa structure est spécifique. C'est une bibliothèque interuniversitaire de proximité, qui a protégé les moyens des sections d'enseignement par rapport aux besoins des sections de recherche.

Sur le campus Saint-Bernard (devenu Jussieu), le doyen et le conseil de la faculté n'avaient pas voulu d'une bibliothèque centrale. L'organisation mise en place lors de la seconde tranche de construction du campus a répondu aux demandes des enseignants et des chercheurs : une bibliothèque de $1^{\mathrm{er}}$ cycle, des bibliothèques d'enseignement et de recherche par discipline, au total 15 bibliothèques. C'était créer une multiplicité de services, petits, moyens ou d'assez grande taille (comme la section mathématiques-informatique enseignement). Ces services inégalement installés sont maintenant en cours de déménagement et de relogement, au fur et à mesure des travaux de rénovation du campus.

C'est une organisation réactive, proche du public, mais plus complexe à faire fonctionner. La bibliothèque ainsi constituée est physiquement et fonctionnellement décentralisée, avec une faible visibilité, des relations extérieures limitées.

La proximité n'a pas induit de coopération documentaire forte avec les UFR, sauf en mathématiques-recherche et sciences de la terre-recherche.

Lors des débats du conseil de la BIU sur l'extension des horaires d'ouverture, les avantages et les inconvénients du modèle ont déjà été soulignés. Dans les contrats actuels des universités figurent des projets de recentralisation. La nouvelle directrice est chargée à la fois de la direction de ce vaste ensemble et d'une mission sur la restructuration physique et la restructuration scientifique de la bibliothèque. La réinformatisation menée à son terme, elle a engagé une politique d'évaluation, d'harmonisation des pratiques de service public et poursuit le développement des fonctions transversales.

Le statut de cette bibliothèque interuniversitaire remonte à 1978. Les universités de Paris 6 et Paris 7 décidèrent alors de coordonner leurs actions pour les disciplines scientifiques au sein de la BIUSJ. Parallèlement Paris 6 et Paris 7 avaient chacune un SCD couvrant d'autres disciplines. 
Cette organisation va être modifiée en accompagnement de l'évolution de la géographie des universités.

Le transfert de l'université de Paris 7 sur la ZAC Paris Rive Gauche (PRG) est préparé. Il sera suivi du réaménagement du campus de Jussieu au profit de Paris 6 et de l'Institut de Physique du Globe (Îlot Cuvier).

Ces perspectives ont amené chacune des parties à définir une organisation spécifique dans les contrats quadriennaux 2001-2004 :

- Paris 6 souhaite regrouper sa documentation au sein d'un SCD unique, comportant quatre pôles : une bibliothèque d'enseignement et deux pôles scientifiques, issus de la BIUSJ, et un pôle santé (issu de l'actuel SCD médical de Paris 6).

- Paris 7 prévoit un secteur scientifique $1^{\text {er }}-2^{\text {ème }}$ cycle dans sa nouvelle bibliothèque centrale (Lettres-Sciences), en construction aux Grands Moulins. Une organisation documentaire recherche sera mise en place au fur et à mesure de l'implantation d'unités de recherche en sciences à PRG. Jusqu'ici le point fort du SCD de Paris 7 était son secteur médical.

Cette évolution entraîne à terme la disparition de la BIUSJ, sinon de toute coopération interuniversitaire. Le conseiller d'établissement a été chargé par la Direction de l'enseignement supérieur d'une mission pour examiner les perspectives de la BIUSJ en ce qui concerne son statut, ses missions et ses collections. Il a rendu son rapport en octobre 2002, en préconisant des lignes de partage des collections de la BIUSJ entre les universités cocontractantes et l'Institut de physique du globe. Des concertations sur ce sujet entre le ministère et les universités de Paris 6 et Paris 7 ont eu lieu à partir de juin 2003.

Paris 7, université pluridisciplinaire comptant 24200 inscrits en 2002-2003, avec $33 \%$ d'étudiants de $3^{\text {ème }}$ cycle, va bénéficier d'une nouvelle bibliothèque centrale. L'université a fait du SCD une priorité dans sa nouvelle implantation.

Ce SCD se trouve donc à la veille d'une mutation considérable, avec l'ouverture prochaine d'une bibliothèque Lettres-Sciences de $11860 \mathrm{~m}^{2}$, aménagée dans le bâtiment des Grands Moulins sur le site Paris Rive Gauche.

Le SCD ne couvrait jusqu'ici que deux grands secteurs disciplinaires. En santé, il regroupe deux bibliothèques de grandes UFR médicales (Xavier-Bichat et Lariboisière-Saint-Louis), la bibliothèque de l'UFR d'odontologie, et deux bibliothèques spécialisées (la bibliothèque de l'Institut d'hématologie de l'hôpital Saint-Louis et la bibliothèque de l'hôpital pédiatrique Robert-Debré). On sait combien étaient limités les moyens documentaires de la section lettres et sciences humaines du SCD : une section de très petite taille, $670 \mathrm{~m}^{2}$ pour 10000 étudiants. Dans ce secteur, deux bibliothèques avaient été récemment intégrées : la bibliothèque de l'UFR d'études anglophones et la bibliothèque de l'UFR géographie, histoire et sciences de la société (GHSS).

Dans le cadre de son université, le SCD pilote une des principales opérations d'U3M : programmation et suivi de la construction d'une nouvelle bibliothèque, mise à niveau du fonds de lettres et sciences humaines, création d'un fonds de sciences. L'objectif est d'offrir 200000 volumes en libre accès. Pour réussir l'opération PRG, le SCD a défini une charte de développement des collections, des programmes d'acquisition par discipline, que l'on a évoqués supra.

On doit noter les points forts de la politique documentaire de l'université Paris 7 :

- l'insertion du SCD dans les structures de l'université, dans l'opération PRG,

- le projet même de nouvelle bibliothèque centrale, appuyé par la priorité donnée aux créations de postes de bibliothèque, l'accompagnement par l'université du travail systématique engagé pour les acquisitions du site PRG,

- des intégrations en médecine (bibliothèque pédiatrique Robert-Debré), puis récemment en lettres et sciences humaines, menées en suivant les procédures régulières, avec transfert ou maintien de moyens des partenaires. C'est une politique réaliste, qui ne sous-estime pas les moyens à fournir pour une intégration, si on veut la réussir,

- une politique d'enquête régulière sur l'état des BUFR en lettres et sciences humaines,

- une aide du $B Q R$ pour les périodiques électroniques. 
Cependant apparaît une zone de flou quant au périmètre du SCD. En santé, il couvre tous les cycles et comprend deux bibliothèques spécialisées de niveau recherche (hématologie, pédiatrie). En lettres et sciences humaines, la nouvelle bibliothèque acquiert pour tous les cycles, sans chercher à intégrer les bibliothèques de recherche.

En sciences, l'équipe du SCD est à créer. Celui-ci doit mettre en place un fonds de $1^{\text {er }}$ et $2^{\text {ème }}$ cycles pour la nouvelle bibliothèque centrale. La documentation recherche devrait se constituer à partir de l'héritage de Jussieu et pourrait relever de bibliothèques associées (telle la bibliothèque de l'Institut Jacques-Monod). Au moment de l'inspection, l'organisation pratique de la documentation recherche (financement, organisation des mètres carrés, conditions d'accueil des étudiants aux niveaux mastère et doctorat) n'apparaissait pas déterminée.

Les conditions de départ sont complexes, mais il importe d'établir tous les liens possibles entre le SCD et l'ensemble de la documentation du secteur scientifique, au fur et à mesure de sa constitution : conseil sur l'organisation des locaux, politique formalisée d'acquisition, catalogue collectif des acquisitions, politique d'accès aux périodiques électroniques, réseau de NTIC, organisation des formations documentaires...

\subsubsection{La lecture publique en Polynésie française}

Une inspection du SCD de l'université de Polynésie française ayant été inscrite au programme de l'IGB, il a paru opportun à l'inspecteur (J.-L. Gautier-Gentès) de saisir l'occasion de ce séjour à Papeete pour s'informer, avec l'assentiment du ministère de la Culture et de la Communication, de la situation de la lecture publique en Polynésie française. Cette évaluation a été conduite en accord avec le ministère de la Culture, de l'Enseignement supérieur et de la Recherche, chargé de la promotion des langues polynésiennes, du Territoire, et sa coopération.

Pour comprendre la situation, il n'est pas inutile de rappeler les données suivantes :

- La Polynésie française est un "Territoire". Elle dispose d'un gouvernement, et d'une assemblée élue. Le président du gouvernement est membre du sénat métropolitain. La présence de l'État est assurée par un haut-commissaire.

Certaines compétences ont été "territorialisées", d'autres non. Il est à noter que des domaines font l'objet d'appréciations divergentes quant aux compétences respectives du Territoire et de l'État.

- Le Territoire se compose de 118 îles réparties en 5 archipels : les îles de la Société, dont les îles-sous-le-vent et les îles-du-vent, parmi lesquelles Tahiti (en fait, une île double) ; les Tuamotu ; les Australes ; les Gambier ; les Marquises. Située sur l'île de Tahiti, Papeete est la capitale politique et administrative de la Polynésie française.

La population n'est que de 233405 habitants (2002), dont 201254 (86\%) dans les îles de la Société et 127635 (54\%) dans l'agglomération de Papeete. Mais l'ensemble du Territoire est grand comme l'Europe. Par exemple, il y a 1500 kilomètres du chef-lieu des Marquises à Papeete.

- Liée à la France, la Polynésie n'en est pas moins inscrite par la géographie et par l'histoire dans deux aires "régionales" : 1) ce qu'il est convenu d'appeler le "triangle polynésien", dont le sommet est constitué par Hawaï, l'angle gauche par la Nouvelle-Zélande et l'angle droit par l'île de Pâques ; 2) un ensemble "Pacifique" comprenant aussi la Micronésie (archipels des Mariannes, des Carolines et des Marshall) et la Mélanésie (Nouvelle-Guinée, îles Salomon, Vanuatu, NouvelleCalédonie, îles Fidji) ; entre les trois sous-ensembles, la frontière serpente.

Comme toujours en pareil cas, l'inscription de la Polynésie française dans ces ensembles est à la fois une réalité ethnique et culturelle et une construction a posteriori, répondant à des besoins et visées d'ordre divers.

- L'équivalent du contrat de plan État-Région est, dans le cas du Territoire, le contrat de développement. Le contrat en cours (le deuxième) venait à échéance à la fin de 2003. Il était susceptible de faire l'objet, comme le précédent, d'avenants; au moment de la visite, les perspectives étaient incertaines sur ce point. 
Les communes ne lèvent pas l'impôt.

Le concours particulier ne s'applique pas.

\section{$>$ La mission de 1986}

Du 5 au 15 février 1986, une mission d'étude a été conduite en Polynésie par le chef du service des bibliothèques publiques de la DLL, en accord avec le Territoire, à la demande du cabinet du ministre de la Culture français et du haut-commissaire de l'époque, particulièrement sensible à la question. Elle a abouti à la rédaction d'un Rapport en vue de la mise en œuvre d'un plan de développement pour le livre, la lecture, les bibliothèques en Polynésie française.

S'agissant des bibliothèques, on retiendra du constat les informations suivantes :

- Absence de bibliothèque municipale à Papeete, la bibliothèque de l'office territorial d'action culturelle (OTAC, aujourd'hui maison de la culture) - insuffisante à tout point de vue - en tenant lieu ainsi que de "bibliothèque générale" pour la Polynésie.

- Absence de BCP.

Au début des années 1980, un "biblionave" desservait plusieurs écoles de Tahiti. Le fonctionnement était assuré par l'OTAC, le pilote étant toutefois pris en charge par l'Éducation nationale. En 1986, ce biblionave ne fonctionnait plus.

Conformément au vœu du haut-commissaire, le plan préconisait la mise en place d'un véritable "réseau de lecture publique". Il prévoyait principalement :

- l'ouverture de bibliothèques communales dans les îles-sous-le-vent, les Tuamotu, les Gambier, les Australes et les Marquises ; la mise en service d'un "bibliotruck" (14) pour Tahiti ; l'OTAC assumerait le rôle de tête de réseau (fourniture et catalogage des documents, fonctionnement du bibliotruck, formation des personnels), le coordonnateur recevant une formation en métropole ;

- la création d'une bibliothèque territoriale, à vocation d'étude et de recherche. Il s'agissait déjà à l'époque d'un "vieux projet".

Ce plan était validé par le haut-commissaire, et le budget nécessaire dégagé ( $2 \mathrm{MF}$ ) à raison de $50 \%$ chacun par le ministère chargé de l'Outre-mer et le ministère de la Culture. L'accord conclu à ce sujet entre l'État, le Territoire et la commune de Papeete était formalisé par une convention signée en 1988 ; elle précisait que le fonctionnement serait à la charge du Territoire et des communes concernées.

Un compte rendu adressé par l'OTAC en 1990 fait état de la création et de l'équipement de 5 bibliothèques (Taravao, Tahiti-Presqu'île ; Ua Pou, Marquises Nord ; Huanine, îles-sous-le-vent ; Rurutu et Tupuai, Australes) ainsi que du renouvellement des collections de la bibliothèque de l'OTAC.

Une douzaine d'années plus tard, il apparaît que ces initiatives sont restées grosso modo sans lendemain et que le constat porté en 1986 - celui d'un sous-développement de la lecture publique - reste valable.

$>$ En 2003

\section{A Tahiti}

S'agissant du secteur culturel, dépendent directement du ministère de la Culture et de l'Enseignement supérieur du Territoire - outre le service de la culture et du patrimoine qui relaie l'action du ministère et les deux académies (tahitienne et marquisienne) - : la maison de la culture ; le musée de Tahiti et des îles et le musée Gauguin; le conservatoire artistique territorial.

La seule bibliothèque publique, sur l'île de Tahiti, reste celle qu'abrite cette maison de la culture, anciennement l'OTAC. Celle-ci est située en bordure de mer, non loin du cœur de Papeete

${ }^{(14)}$ Les truck(s) sont les bus locaux. 
où voisinent, de l'autre côté de la route qui longe le littoral, commerces et bâtiments administratifs.

Ce qu'on nomme et nommera ici bibliothèque se compose en réalité de 3 espaces séparés : une bibliothèque pour adultes et adolescents de $235 \mathrm{~m}^{2}$, une bibliothèque pour enfants de $155 \mathrm{~m}^{2}$ et une discothèque-vidéothèque de $42 \mathrm{~m}^{2}$. Soit un total de $432 \mathrm{~m}^{2}$ d'espaces publics.

Sur une douzaine de personnes gérant et animant les activités dites permanentes du centre, 5 sont employées à la bibliothèque.

Sont à porter au chapitre des points positifs :

- L'agrandissement et la rénovation dont ont fait récemment l'objet les locaux de la bibliothèque des adultes : extension de $90 \mathrm{~m}^{2}$, climatisation, éclairement amélioré, création d'un espace de travail pour les étudiants et d'un petit salon de lecture.

- Des plages d'ouverture hebdomadaire (44 heures) très supérieures à la moyenne des BM françaises $(18 \mathrm{~h})$. Il faut toutefois remarquer que le centre est fermé le samedi.

- Les subventions allouées depuis quelques années par le ministère de la Culture du Territoire, et qui s'ajoutent au budget dégagé par le centre.

- L'ouverture, en mars 2002, d'un cyber-espace $\left(91 \mathrm{~m}^{2}\right)^{\left({ }^{15}\right)}$.

- L'atout que représente la présence de la bibliothèque dans ce centre polyvalent. En effet, celui-ci comprend, outre le cyber-espace, une salle de projection, une salle de cours et une salle d'exposition. Sont proposés des cours de langues pour adultes et enfants (anglais, reo ma'ohi ${ }^{(16)}$ ), des ateliers d'arts plastiques, des projections hebdomadaires de films pour les enfants et les adolescents, etc.

Avant la fin de l'année, devait être installée une borne permettant de visionner des documentaires consacrés à la Polynésie depuis les années 1940.

Par ailleurs, la maison de la culture dispose depuis peu d'un site Internet. Une partie est consacrée à la bibliothèque et au cyber-espace. Il est possible d'accéder par cette voie à une liste des ouvrages relatifs à la Polynésie et au Pacifique.

La bibliothèque n'en reste pas moins cruellement sous-dimensionnée. C'est le cas quelle que soit la population prise en compte :

- celle de Papeete, soit 26181 habitants (2002), la surface recommandée en pareil cas se situant autour de $2000 \mathrm{~m}^{2}$ (SHON) ;

- a fortiori, celle de l'agglomération de Papeete, soit 127635 habitants (2002). Cette population justifierait l'existence d'une centrale d'au moins $5000 \mathrm{~m}^{2}$ et de plusieurs annexes.

Le mobilier est vétuste.

L'offre en documents est modeste. Elle représentait en effet en 2002, pour les livres, 10405 ouvrages pour les adultes et 6765 ouvrages pour les enfants; pour la discothèque, 1480 CD ; pour la vidéothèque, environ 1200 vidéocassettes et 170 DVD.

La bibliothèque n'est pas informatisée.

Le personnel ne présente pas de qualification spécialisée.

Collectivités comprises, le total des usagers inscrits était d'environ 2500.

\footnotetext{
(15) Il s'agit d'un espace culture multimédia.

(16) Ce terme générique comprend les cinq idiomes, correspondant aux cinq archipels de la Polynésie française, auxquels a donné naissance une langue qui passe pour avoir été originellement unique.
} 
Rapporté à la population de Papeete, ce chiffre représente $9,5 \%$ (17); on sait que la moyenne nationale française tourne autour de $18 \%$. Rapportée à la population de l'agglomération, la proportion des inscrits chute à $1,9 \%$.

\section{Dans l'agglomération} culture.

Il n'est pas signalé d'autres bibliothèques publiques à Tahiti que celle de la maison de la

Celle-ci reçoit des classes. Mais, compte tenu de l'éloignement et d'un réseau de transports collectifs encore insuffisant, il ne peut pas s'agir pour les jeunes de l'agglomération d'une bibliothèque fréquentée à l'égal d'un établissement de proximité. Pour tout service public de lecture, ceux-ci ne se verront donc proposer que les BCD et les CDI.

Locaux vétustes; collections pauvres et défraîchies ; quelques ordinateurs, mais anciens et, au demeurant, en panne depuis un certain temps : tel est le constat auquel a conduit la visite d'un $\mathrm{CDI}$, celui du collège d'Arué. Or, conformément à la demande formulée pour le choix de l'établissement visité, ce CDI est représentatif de l'ensemble. Il y a des exceptions; elles confirment la règle.

Il est d'autre part à noter que la bibliothèque de l'université a pris la décision de développer, à l'intention de ses usagers, un fonds de culture générale. Cette orientation s'observe aussi en métropole. Là où existent des bibliothèques de lecture publique conséquentes, elle a souvent pour objet de pallier l'excentration des campus. C'est aussi le cas à Papeete (l'université est à $10 \mathrm{~km}$ de la ville). Où, toutefois, la pauvreté de la bibliothèque de la maison de la culture constitue un motif supplémentaire de prendre ce parti.

\section{Au-delà de Tahiti}

Il est apparu que les bibliothèques nées du plan de 1986 étaient restées à peu de choses près dans l'état où elles se trouvaient lors de leur création. En effet, il ne suffisait pas de les ouvrir, il fallait encore faire en sorte qu'elles fonctionnent.

Les jeunes étant réputés disposer des $B C D$ et $C D I$, ces bibliothèques avaient été conçues pour les adultes. C'était probablement une erreur.

\section{$>$ Un projet ancien : une médiathèque à Papeete}

Le projet de construction d'une bibliothèque à Papeete est ancien. Il s'alimente à deux besoins plusieurs fois mis en évidence: celui d'une bibliothèque publique et celui d'une bibliothèque d'étude et de recherche orientée vers la Polynésie et le Pacifique; celle-ci constituerait une sorte de bibliothèque nationale.

Signée en 1993 pour s'achever en 1995, mais reconduite jusqu'en 1998, une convention de développement culturel passée entre l'État et le Territoire prévoyait de conduire des études en vue de la création d'un centre culturel, d'une bibliothèque centrale et d'une cité des métiers.

En 1998, le comité paritaire État-Territoire constatait l'abandon du projet et les crédits délégués étaient reversés à l’État.

Pour autant, le projet d'une bibliothèque reste d'actualité. Et il s'inscrit toujours dans le cadre indiqué au cours des années 1990 : celui de l'espace culturel Vaiami.

Il s'agit de créer ce centre dans les locaux que l'hôpital du même nom a libéré depuis peu, comme prévu, mais avec retard. Il se trouve non loin du quartier administratif. Les bâtiments ne

\footnotetext{
(17) Ce chiffre ne comprend pas les utilisateurs du cyber-espace, pour ceux d'entre eux qui ne fréquente pas la bibliothèque au sens où celle-ci a été définie. Ouvert en mars 2002, ce service a reçu plus de 5500 visites cette année-là.
} 
sont pas en bon état ; la question de savoir s'ils seront réutilisés, en totalité ou en partie, n'est pas tranchée.

Le centre Vaiami comprendrait un musée ethnographique, un espace historique, une pinacothèque, un pôle "bibliothèque publique de prêt", des salles de spectacle et une salle d'exposition temporaire.

Représentant $4200 \mathrm{~m}^{2}$, le pôle bibliothèque se décomposerait comme suit :

- la bibliothèque proprement dite $\left(3400 \mathrm{~m}^{2}\right)$, comprenant, d'une part, une bibliothèque centrale de $1600 \mathrm{~m}^{2}$ située dans l'espace Vaiami, d'autre part, 6 annexes de $300 \mathrm{~m}^{2}$ chacune, réparties à raison de 5 à Tahiti et une sur l'île voisine de Moorea;

- dans le centre Vaiami, un centre de documentation sur la culture polynésienne $\left(800 \mathrm{~m}^{2}\right)$.

Si le principe de la création du centre Vaiami a été validé par la présidence du Territoire, il n'en est pas encore de même de son contenu.

\section{$>$ Observations}

1. La lecture publique est, en Polynésie française, en situation de sous-développement. Ce constat n'est pas nouveau ; il est régulièrement porté depuis au moins les années 1960, qui voyaient un inspecteur d'académie écrire au ministre de l'éducation : "L'absence de cet instrument essentiel de culture que représente une bibliothèque publique se fait durement sentir".

Papeete attend toujours sa bibliothèque. Et les autres îles, de bénéficier de même d'un service de lecture publique.

En effet, si le plan mis en place dans les années 1980, qui prenait en compte l'ensemble du Territoire, a connu un début de réalisation, il est resté sans lendemain, faute qu'ait été résolue en particulier la question du fonctionnement des équipements créés.

La Polynésie, entend-on souvent, est une civilisation de tradition orale. Si elle veut valoir objection au développement de la lecture publique, cette objection n'est pas recevable. Primo, le développement social, économique et culturel de la Polynésie, tel qu'il est engagé, passe par la maîtrise des textes et des images, ceux qu'on trouve dans les livres mais aussi sur les écrans des ordinateurs, supports qui sont désormais tous pleinement du ressort des bibliothèques.

Secundo, entre la notion de lecture publique, telle que la représentent les bibliothèques, et celle de civilisation orale, il n'existe aucune incompatibilité. Loin d'éteindre l'oralité, plus généralement les traditions, les bibliothèques sont à même de les recueillir, de les préserver, de leur offrir de nouvelles chances.

Sous diverses formes, le conte et le théâtre ont bien entendu leur place dans les bibliothèques. C'est aussi le cas de la danse et des arts plastiques, qui tiennent, dans la culture polynésienne, le rang que l'on sait.

D'un vivier potentiel de producteurs et de récepteurs de textes, témoignent des maisons d'édition locales, des écrivains talentueux et qui, tôt ou tard, se feront reconnaître bien au-delà du Territoire, des jeunes qui, en dépit de la maigreur de l'offre, persistent à fréquenter les CDI et la bibliothèque de la maison de la culture.

2. Le développement de la lecture en Polynésie ne dépend pas seulement de celui des bibliothèques. Il suppose, pour les livres venant du dehors, l'absence de surcoût. Il passe aussi par la promotion, en amitié avec la langue française, du tahitien ${ }^{(18)}$.

Mais le développement de la lecture en Polynésie passe aussi par les bibliothèques, service public gratuit ou à tarification modérée. (D’autant plus, tant que les livres provenant de métropole vaudront, dans les librairies, deux fois plus cher qu'en métropole.)

\footnotetext{
(18) Depuis 1980, le tahitien est, conjointement avec le français, la langue officielle de la Polynésie française. En 2000, a été instaurée une journée annuelle du reo ma'ohi. Des ouvrages en polynésien sont publiés par exemple par le Centre territorial de recherche et de documentation pédagogique.
} 
3. En l'état, les projets du Territoire soulèvent plusieurs questions.

Le nouvel équipement est appelé à assumer deux grandes fonctions: une fonction de lecture publique ; une fonction d'étude et de conservation, autour de la Polynésie.

S'agissant des fonds polynésiens, le rapport de 1986 faisait état de collections privées, du fonds historique des archives territoriales, de fonds dans les bibliothèques des missions catholiques et évangéliques. "C'est probablement près de 50000 ouvrages sur la Polynésie et le Pacifique", concluait-il, "qu'il serait possible de rassembler dans une bibliothèque territoriale à vocation de conservation, d'étude et de recherche". Concernant le fonds des archives territoriales, il est bien prévu qu'il rejoigne la future bibliothèque.

La fonction lecture publique en comprend elle-même deux : il s'agit, d'une part, de desservir Papeete, d'autre part, de jouer le rôle de tête de réseau pour l'agglomération. En outre, le nouvel équipement pourrait être appelé à jouer le rôle de tête de réseau pour l'ensemble du Territoire.

Pour assumer ces fonctions, les $1600 \mathrm{~m}^{2}$ prévus représentent une surface évidemment insuffisante. Il est rappelé que la seule desserte de Papeete justifierait une surface de $2000 \mathrm{~m}^{2}$.

Par ailleurs, les 6 bibliothèques annexes dont la création est incluse dans le projet du centre Vaiami seraient situées pour 5 d'entre elles à Tahiti, la sixième se trouvant sur l'île voisine de Moorea. La question de la desserte de l'ensemble du Territoire reste donc entière.

Pour desservir les autres îles, et apporter un concours nécessaire à celles qui se doteraient d'une bibliothèque, il n'existe toujours pas en Polynésie de bibliothèque de type BDP. C'est pourtant bien un tel dispositif qui s'imposerait.

Pour le mettre en place, la moindre des difficultés n'est pas celle des possibles relais locaux. En effet, parmi ceux-ci, on trouve soit les nombreuses confessions religieuses qui se partagent les îles, et ne présentent donc pas les garanties de règle en matière de laïcité, soit les enseignants, avec les mérites mais aussi les limites que comporte la façon dont ils envisagent usuellement la lecture.

Les questions qui viennent d'être formulées ne constituent pas une récusation des projets actuels du Territoire - au contraire, il faut se féliciter que celui-ci les nourrisse. Elles désignent des problèmes qu'il est possible et souhaitable de se poser à ce stade, et qui ne sont pas sans solutions.

Encore faut-il, pour rechercher et trouver ces solutions, que la volonté politique nécessaire aille au bout de sa logique. Cette volonté ne saurait émaner d'abord que du Territoire.

Mais elle aura d'autant plus de chances de porter ses fruits que l'État la stimulera et la relayera. De ce point de vue, un éventuel concours financier de l'État n'apporterait pas au Territoire toute l'aide dont il a besoin. Cette aide doit aussi revêtir la forme de l'expertise et du conseil.

État et Territoire, l'enjeu de leur nécessaire collaboration pourrait être résumé comme suit : sous couleur de "tradition orale" et de développement économique, les Polynésiens doivent-ils se voir exclus du droit à l'égalité devant l'offre des bibliothèques publiques ? Sont-ils des citoyens à part entière ? Ou l'industrie et le commerce d'une part, les chants et danses d'autre part, conjoints sous le signe du tourisme, doivent-ils constituer leur seul horizon? 


\subsubsection{Thèmes transversaux}

\subsubsection{Le patrimoine imprimé}

\section{$>$ Les bibliothèques municipales}

La liste des inspections et visites montre que la situation du patrimoine imprimé des bibliothèques municipales a été fréquemment examinée en 2003.

Outre les interventions de J.-M. Arnoult, pour résoudre des problèmes de conservation, plusieurs missions avaient en ce domaine un objectif positif : préparer le relogement des fonds patrimoniaux à Bourges ou le traitement du fonds ancien à Mantes (inventaire, conservation, catalogage), examiner avec la municipalité et le chapitre les conditions d'un transfert de la bibliothèque de la cathédrale de Salins à la bibliothèque de la ville, faire des propositions sur la conservation et la valorisation de la bibliothèque bénédictine de Saint-Mihiel, qui joint attrait architectural et qualité des collections...

Mais dans d'autres cas l'Inspection a dû signaler des difficultés : solution de conservation provisoire d'une partie des fonds patrimoniaux ou mauvaises conditions de conservation, désordre des collections, ou encore manque de personnel spécialisé dans un établissement aux collections de premier rang. Pour une ville, l'inspecteur a signalé l'état d'abandon des collections patrimoniales, la forte différence de traitement entre patrimoine imprimé et patrimoine architectural dans un même lieu.

La réalisation d'équipements destinés à la diffusion du livre et au développement de la lecture publique a parfois fait négliger le patrimoine, sa conservation, son déploiement dans des locaux adaptés. Il serait temps de reprendre cette question dans bon nombre d'équipements et de collectivités.

Dans le même domaine des collections municipales, l'examen en 2003 d'un désherbage mal conduit amène l'Inspection à rappeler que ces questions doivent être traitées avec méthode. L'élimination des documents après désherbage de la collection reste une opération à risque. Il est plus que jamais nécessaire de suivre les précautions d'usage et de remplir scrupuleusement les procédures juridiques.

Deux inspections ont conduit à examiner d'autres aspects du patrimoine imprimé des bibliothèques : le rôle important que peut mener en la matière une bibliothèque départementale de prêt (la Médiathèque départementale de la Loire) ; l'enrichissement patrimonial des SCD, à propos du projet de dépôt de la bibliothèque marxiste auprès de l'Université de Paris XIII.

\section{$>$ Conservation et mise en valeur du patrimoine : l'échelon départemental}

Depuis quinze ans, une bibliothèque départementale de prêt, la Médiathèque départementale de la Loire (MDL), assure une fonction patrimoniale. Une des missions données à l'Inspection en 2003 a permis de faire le bilan de cette action originale, analogue à la conservation départementale des musées et de l'inventaire.

Une convention entre l'État et le Conseil général de la Loire, concernant le plan de développement de la lecture publique dans le département, a permis le lancement de l'opération. En octobre 1987, un secteur "patrimoine du livre" a été créé au sein de la MDL. Il était doté d'un poste de bibliothécaire spécialisé, titulaire du DSB. L'objectif était de répondre aux problèmes de conservation et de mise en valeur du patrimoine imprimé que rencontraient des communes petites ou moyennes.

Le bilan que l'on peut dresser comporte des points forts. Le chargé de mission au patrimoine a assuré le sauvetage (déménagement, nettoyage, désinfection) de fonds anciens - dont la situation préoccupante avait été signalée à plusieurs reprises par l'Inspection, sans réel effet.

Pour la mise en valeur des fonds, dès lors qu'ils se trouvaient dans des locaux sains et adaptés, il a assuré la publication d'une série de catalogues, organisé des expositions, préparé un projet de numérisation. S'y ajoutent le suivi de la conservation des collections sauvegardées, l'appui aux programmes de microfilmage, la préparation de dossiers de restauration. Cette action 
patrimoniale a trouvé assez tôt l'appui de l'Université de Saint-Étienne (Institut Claude Longeon), dont un projet de recherche porte sur le patrimoine régional. L'aide universitaire a pris trois formes : aide intellectuelle aux projets, études sur les fonds anciens foréziens (présentées dans le Bulletin du Patrimoine de la MDL), publication par les presses de l'université de catalogues imprimés de fonds anciens de la Loire.

Cette expérience montre que l'échelon départemental est très propice au repérage de fonds (au delà des bibliothèques municipales) et à l'action appliquée. Moins loin que la région, le département est sensible aux petits fonds et aux pays. Au delà du registre habituel des actions régionales (répertoire des collections, conseil, incitation à la coopération), c'est un échelon qui permet une action "de fond" (inventaire, catalogage, opérations de conservation), un suivi avec retour périodique sur les lieux. Le département peut en outre prendre le patrimoine en compte dans une politique d'aménagement du territoire, à la fois culturelle et touristique.

L'expérience montre aussi que l'ancrage d'un secteur patrimoine dans une BDP ne va pas de soi. C'est un secteur à construire avec une série de communes et avec d'autres services du département.

La barre théorique de l'action en faveur des communes de moins de 10000 habitants n'a pas de sens pour le patrimoine et a très vite sauté. Le travail du secteur patrimoine n'entre pas dans la relation permanente de la MDL avec la majorité des communes du réseau. En revanche, le patrimoine fait partie de sa "politique" : relations de la médiathèque départementale avec les bibliothèques municipales des villes moyennes (et spécialement celle où elle est implantée), projets d'intercommunalité pouvant avoir une dimension patrimoniale.

L'aide fournie aux communes au titre du patrimoine n'est pas négligeable, mais n'a pas par nature une forte visibilité, ni un effet d'entraînement. La relation entre département et communes doit être formalisée (objectifs, apports, répartition de rôles, bilans périodiques). Cela suppose une réflexion au niveau du département, après avoir identifié les fonds anciens : quelles missions afficher, quel périmètre envisager (communes, associations et autres organismes possédant des fonds anciens?), quels objectifs à long terme après la sauvegarde (relais par les communes, conservation centralisée, suivi sur place en compensant les faiblesses des propriétaires des fonds) ?

Une relation a été construite entre la médiathèque départementale et les archives départementales ${ }^{(19)}$. En effet, en principe, une bibliothèque départementale n'a pas de rôle de conservation, à la différence d'une bibliothèque municipale ou des archives départementales. Dans un département, ce sont habituellement les archives qui ont une approche patrimoniale des communes et qui sont perçues comme un interlocuteur patrimonial par celles-ci. De manière très pratique, ce sont elles qui disposent de capacités éventuelles d'accueil de fonds (magasins), de compétences et d'outils techniques (tests des conditions climatiques, par exemple). Il semble bien qu'un poste départemental "patrimoine imprimé" doive se situer techniquement, dès le départ, par rapport à la bibliothèque départementale et par rapport aux archives départementales.

L'initiative du département de la Loire a intéressé d'autres zones géographiques (département de l'Ain - dont les archives ont recueilli des fonds de bibliothèques en déshérence, DRAC PACA à propos de fonds anciens des Hautes-Alpes, diverses BDP). En fait, l'opération n'a pas été étendue au sein de la région Rhône-Alpes, ni dupliquée à l'extérieur.

Cependant, l'efficacité et l'intérêt du niveau départemental doivent être soulignés, en regard des capacités et des investissements des petites villes. Souvent, les communes petites et moyennes, responsables de collections patrimoniales, municipales ou d'État, n'ont pas de personnel professionnel formé, A ou B, affecté aux fonds anciens. Là comme ailleurs, le capital de compétences qu'avait apporté l'option Livre ancien du CAFB s'est effrité. Mais le problème est moins l'offre de formations que la capacité ou la volonté d'investissement des communes dans cette compétence patrimoniale (en concurrence avec les compétences nouvelles qu'elles doivent assurer dans d'autres secteurs).

\footnotetext{
(19) Les relations entre bibliothèques et archives ont fait l'objet d'une étude d'Albert POIROT, sur laquelle nous nous appuyons : "Les archives dans les bibliothèques ", dans Bulletin des bibliothèques de France, 2001, $\mathrm{n}^{\circ} 2$, notamment $\mathrm{p}$. 12 14. On trouvera dans le même numéro du BBF le point de vue des archives (A. PLAYOUST, "Les bibliothèques dans les services d'archives").
} 
Dès lors, ces fonds patrimoniaux sont au mieux conservés, sans interlocuteur pour les utilisateurs scientifiques, avec de faibles possibilités de mise en valeur (catalogage, expositions...), sans capacité d'élaborer des dossiers d'acquisition à l'intention du Fonds régional d'acquisition pour les bibliothèques (FRAB), quand la région en comporte un, ou des dossiers de restauration à l'intention du Conseil national scientifique du patrimoine des bibliothèques publiques (CNSPBP).

Dans ce contexte, des politiques départementales du patrimoine écrit pourraient être très efficaces, en liaison avec le niveau régional (Associations régionales de coopération, DRAC), pour garantir les bases nécessaires pour la conservation et la mise en valeur du patrimoine sur la longue durée : des locaux adaptés, un inventaire réalisé, le suivi de personnel professionnel compétent.

\section{$>$ Le dépôt de la Bibliothèque marxiste de Paris}

Une convention de dépôt entre le Parti communiste et l'université de Paris XIII est en cours de préparation pour le dépôt de la Bibliothèque marxiste de Paris au SCD de cette université.

La Bibliothèque marxiste, fondée en 1955 par des membres du Comité central était gérée depuis 2001 par l'association "Archives du communisme français". Le Parti communiste français a souhaité une évaluation des conditions de conservation, ainsi que des moyens nécessaires à la mise en valeur de ces fonds dans la perspective d'une insertion dans un réseau documentaire.

Cette bibliothèque, qui a connu des vicissitudes diverses, est abritée depuis 2001 dans des locaux où la sécurité des collections est tant bien que mal assurée, mais où aucun travail sur les collections n'est possible.

Les fonds proviennent d'origines diverses : dons de dirigeants et de militants ; services de presse ; dépôts volontaires des maisons d'édition proches du PCF ; échanges internationaux avec les partis frères. Cet ensemble, composé principalement de documents sur le mouvement ouvrier, les différents courants socialistes et anarchistes du $19^{\text {ème }}$ siècle, la pensée marxiste, comporte de nombreuses pièces rares ou uniques, ainsi qu'un fonds important de manuscrits historiques ou littéraires (archives, correspondances de personnalités politiques, archives littéraires de Henri Barbusse et Paul Vaillant-Couturier). Quantitativement, la collection de livres compte 30000 ouvrages environ, publiés entre 1789 et 2000. La partie ancienne illustre les mouvements de 1789 , 1848 et 1871 . S'y ajoutent 15000 brochures, des $19^{\text {ème }}$ et $20^{\text {ème }}$ siècles et des séries de périodiques sur papier et microfilm.

M. Alain Neuman président de l'Université de Paris XIII et la directrice du SCD ont évalué l'intérêt du point de vue de la carte documentaire des universités parisiennes, du dépôt d'un fonds patrimonial de cette importance dans une université du nord de l'île-de-France.

Cette solution contribue à l'enrichissement de la documentation historique du SCD et renforce le rôle de recherche de l'université. Dans un domaine où les sources s'ouvrent à peine, l'université dépositaire pourrait jouer un rôle pionnier dans le développement des études grâce à la valorisation documentaire menée par le SCD. De nombreux chercheurs ont appuyé le projet. L'accueil du fonds suppose des aménagements. Il pourrait être appuyé par une contribution des collectivités locales.

Les archives du Parti communiste font l'objet parallèlement d'un dépôt aux archives départementales de la Seine-Saint-Denis, localisation proche de l'Université de Paris XIII. Le rapprochement géographique de ces deux fonds complémentaires serait favorable à la recherche.

L'entrée d'un tel fonds conduit à souligner le rôle que peuvent jouer les universités et leurs SCD dans la conservation et la mise en valeur du patrimoine imprimé - qui comprend à la fois les fonds anciens rares et précieux et les fonds spécialisés, constituant une source importante de la recherche.

Si elle n'est pas fréquemment soulignée, la présence de collections particulières et de fonds spécialisés n'est pas rare dans les bibliothèques universitaires. On connaît la très grande richesse des bibliothèques interuniversitaires parisiennes.

Mais l'accueil et le développement de fonds spécifiques concernent nombre de SCD dans différentes régions. Un exemple remarquable a été la constitution de la Bibliothèque de la Société industrielle de Mulhouse en section du SCD de l'Université de Haute-Alsace. 
Au cours des missions de 2003, l'Inspection a eu connaissance de divers fonds spécialisés :

- Au SCD de Besançon, fonds Rhodia.

- Au SCD d' Angers, fonds Anthony Burgess, André Dhôtel, Julien Gracq, Octave Mirbeau, Michel Tournier (avec un grand nombre de documents personnels), Henri Petit (journalisme, sciences sociales et politiques), Serge Brindeau (poésie), fonds de l'école de Rochefort (poésie).

Ce SCD abrite aussi les archives de la protection de l'enfance (éducation surveillée), les fonds de l'ancien centre international de l'enfance et de la famille, transférés de Longchamp à Angers fin 1999, après accord du conseil d'administration du CIDEF et du conseil d'administration de l'université. Ce sont des collections d'une grande richesse sur l'enfance dans les pays développés et surtout dans ceux du Tiers-monde, renforçant un pôle documentaire constitué depuis quelques années autour de la psychologie de l'enfant, des problèmes de l'adolescence et de la pédiatrie.

\subsubsection{Le harcèlement moral}

En 2003, l'Inspection a été amenée à trois reprises à examiner la question du harcèlement moral - de manière spécifique, ou dans le cadre d'un contrôle plus général. Dans un cas, une demande de protection juridique a été formulée par un avocat face à un harcèlement présumé. Une inspection conjointe IGAENR-IGB a été demandée sur ce sujet par le directeur de cabinet du ministre de la Jeunesse, de l'Éducation nationale et de la Recherche. Après examen d'un ensemble abondant de griefs, de sources et témoignages, le harcèlement moral n'est finalement pas apparu prouvé. Mais des remarques et recommandations ont dû être faites à l'établissement et au rectorat concernés en ce qui concernait la pérennisation d'une situation irrégulière. Dans le second cas, le plaignant avait constitué un dossier. Grâce à une médiation, engagée suffisamment tôt, le conflit a trouvé une solution. Dans le troisième cas, la possibilité d'une plainte a été évoquée lors d'une inspection et a été examinée, mais la plainte n'a pas réellement pris forme.

La loi de modernisation sociale du 17 janvier 2002 a fait apparaître la notion de "lutte contre le harcèlement moral au travail" (articles 168 à 180).

Cette même loi a prévu la répression du harcèlement moral à trois niveaux : au plan disciplinaire, au civil dans le cadre du Code du travail, et enfin dans le Code pénal (article 222-33-2). Au pénal, le harcèlement est passible d'une peine d'un an d'emprisonnement et d'une amende de 15000 euros.

La lutte contre le harcèlement moral a été introduite dans le statut des fonctionnaires (article 6 quinquies) : " aucun fonctionnaire ne doit subir les agissements répétés de harcèlement moral qui ont pour objet ou pour effet une dégradation des conditions de travail susceptible de porter atteinte à ses droits et à sa dignité, d'altérer sa santé physique ou mentale ou de compromettre son avenir professionnel...". II n'y a cependant pas de texte d'application, qui organise par exemple la médiation applicable aux emplois du secteur privé.

Dans un contexte économique dur, la notion de harcèlement moral a des échos médiatiques. Chaque année des ouvrages sont publiés sur le sujet. Plusieurs sites Internet y sont consacrés. C'est cependant un concept qui fait débat, car la notion de harcèlement est encore floue et globalisante. Elle introduit dans les conflits au travail un vocabulaire psychologique et reporte sur la justice des problèmes réels qu'on ne sait pas bien résoudre.

Dans les faits, la perspective de judiciarisation d'un conflit oblige l'administration à une attention particulière. D'un côté, toute action en justice fragilise l'administration, accusée de défauts de gestion. Celle-ci doit être exigeante vis-à-vis des gestionnaires. D'un autre côté, l'accusation de harcèlement moral est grave. L'administration doit vérifier avec attention qu'une telle accusation est portée à bon escient.

Les effets de la loi du 17 janvier 2002 ont-ils été annoncés aux responsables d'établissement ? A l'examen de deux de ces dossiers, les inspecteurs se sont demandés si l'importance des nouveaux textes avait été soulignée, si l'étendue des questions que soulevait une plainte pour harcèlement moral était bien appréciée. Dans le troisième cas, le directeur de bibliothèque était parfaitement informé. 
Il est probable que des plaintes pour harcèlement moral vont apparaître épisodiquement dans le monde des bibliothèques. Sur ce sujet, l'Inspection juge nécessaire de souligner plusieurs faits :

- Une notation et une évaluation bien menées devraient pouvoir prévenir nombre de ces conflits. Or les difficultés relationnelles - forte composante des dossiers de harcèlement - paraissent souvent occultées ou sous-estimées dans les dossiers administratifs (lus, il est vrai, a posteriori). Il est possible que, sur de telles questions, des tutelles locales ou des responsables de bibliothèques redoutent la transparence - ou la tension que crée une mise à plat périodique. C'est un mauvais calcul à long terme.

Dans tous les cas, le dossier administratif doit être complet - mais sans pièces étrangères à sa nature. Faut-il de plus préciser qu'il doit être cohérent, et ne pas comporter, par exemple, une proposition de promotion au grade supérieur et une demande de mutation d'office pour trouble manifeste au fonctionnement du service, établies successivement, à trois mois d'intervalle, par le même directeur de bibliothèque à propos du même agent ?

- La lutte contre le harcèlement moral, prévue par la loi, introduite dans le statut des fonctionnaires, crée des obligations aux établissements et aux tutelles : une gestion qui ne dégrade pas les conditions de travail, qui n'altère pas la santé... Elle entraîne une nouvelle approche des conflits entre établissement et personnel. Elle donne un réel pouvoir au plaignant, quel que soit l'avis de l'établissement sur ses qualités professionnelles. Il ne faut en aucune manière négliger ce type de dossier. Il faut prendre le temps d'écouter l'agent qui se dit victime.

En revanche, le harcèlement moral est une accusation qui doit être solidement motivée. Elle ne peut être portée à la légère. Il est hors de question de prendre au sérieux un discours sur le harcèlement, sans que les plaignants se soient déclarés, sans qu'un argumentaire détaillé étaye leur plainte.

- Suivant la littérature juridique, l'accusation de harcèlement moral se fonde sur l'existence d'agissements fautifs, répétés dans le temps, ayant eu des conséquences sur les conditions de travail et la santé de l'intéressé.

Lorsque une plainte pour harcèlement moral fait l'objet d'une inspection, les inspecteurs sont obligatoirement attentifs à l'ensemble des griefs exprimés par le plaignant. Ils doivent entrer dans le détail des faits, obtenir des documents et des témoignages, afin d'identifier les faits susceptibles de faire partie d'un processus de harcèlement moral, sous réserve de leur caractère intentionnel, et les faits qui doivent être écartés ; afin de distinguer ce qui relève du harcèlement éventuel d'une personne ou de conditions de travail communes à plusieurs. Si un chef d'établissement est incriminé, il doit être en mesure d'établir de son côté un dossier complet de pièces en réponse et un mémorandum sur sa gestion.

A l'occasion de ce type d'affaire, un élément nouveau est apparu : l'abondance des courriers électroniques, sources proposées par les parties, sans que les inspecteurs puissent être sûrs du caractère complet de l'information apportée. La confidentialité du courriel privé dans un cadre professionnel a été défendue par la Cour de cassation en 2001, avis suivi par la CNIL.

- L'instruction du dossier doit être aussi nourrie que possible par toutes les parties. Tous les faits pourront être réexaminés par des instances juridiques, hors du cadre administratif et professionnel des bibliothèques. 


\subsection{LES BIBLIOTHEQUES TERRITORIALES ET LA DECENTRALISATION : ETAT DES LIEUX}

\subsubsection{Introduction}

\subsubsection{Origine et limites de ce travail}

A l'origine de cette partie, se trouve une communication présentée par un des membres de l'Inspection (J.-L. Gautier-Gentès) lors de la sixième conférence internationale Les bibliothèques publiques dans la nouvelle Europe.

Cette conférence qui s'est tenue à Varsovie les 20 et 21 novembre 2003 avait pour thème : "Bibliothèques publiques et stratégies de développement après l'élargissement de l'Union européenne". A l'Inspection générale, il avait été demandé de traiter - sous le rapport des bibliothèques publiques - de l'expérience française en matière de décentralisation.

Dans un pays où, soutenues par l'État sous le régime socialiste, même si c'était à la façon dont la corde soutient le pendu, les bibliothèques publiques, désormais laissées à la libre appréciation des collectivités locales, ont le sentiment d'être devenues fragiles; dans un pays où, comme dans d'autres qui ont connu la même histoire au sein de la même zone géographique, les bibliothécaires se demandent s'ils sont à la veille du naufrage ou de l'essor - dans ce pays, donc, il est apparu aux organisateurs de la conférence que la dite expérience française en matière de décentralisation, expérience qui a consisté à tenter de concilier action de l'État et responsabilité des collectivités locales, était de nature à intéresser les bibliothécaires et responsables des politiques en matière de bibliothèques. C'est en effet ce qui s'est produit ; le dispositif du concours particulier, notamment, a retenu l'attention des auditeurs.

D'une manière générale, il ne faut pas sous-estimer l'attrait qu'est susceptible d'exercer le "système français", la conception française de la lecture publique comme relevant de l'action publique dans des régions du monde où, dégagés du totalitarisme, tous les professionnels n'ont pas nécessairement envie de jeter le bébé avec l'eau du bain : les bibliothèques publiques comme services publics avec l'emprise sur les publications et les acquisitions d'une idéologie d'Etat.

Entre la bibliothèque socialiste, courroie de transmission du marxisme-léninisme, et la bibliothèque libérale, grignotée par Coca Cola, il semble à des bibliothécaires soulagés d'être débarrassés de la première sans pour autant trouver la seconde indépassable qu'il y a - notamment - la bibliothèque française. augmentée.

De l'exposé précité, les pages qui suivent constituent une version considérablement

Pour autant, elle ne saurait être considérée autrement que comme partielle et provisoire. Le sujet qui se cache derrière celui de la décentralisation, le vrai sujet, c'est en effet celui de la politique de l'État en matière de bibliothèques depuis le début des années 1980 , de l'idée que l'État s'est fait de son rôle vis-à-vis des collectivités locales et des conclusions qu'il en a tirées ou, pour diverses raisons, n'en a pas tirées.

Or, cette histoire reste à écrire pour peu qu'on puisse, d'une part, disposer de tous les matériaux nécessaires (et en particulier, de chiffres fiables), et qu'on veuille d'autre part s'écarter de l'hagiographie. On ne pose ici, on ne pouvait ici poser que des jalons.

Pour diverses raisons, nous avons orienté le projecteur vers les services du ministère de la Culture. Il y aurait lieu de s'intéresser aussi, en particulier, aux associations professionnelles. En effet, leur étude est susceptible de livrer au moins deux types d'informations. En premier lieu, pour ou contre selon les dossiers et les périodes, plus ou moins écoutées et, quand elles étaient écoutées, entendues, elles ont été parties prenantes à la décentralisation ; elles ont contribué à faire ce qu'elle a été - et ce qu'elle n'a pas été - et relèvent bien entendu pleinement, à ce titre, d'une histoire de ce phénomène et de la période considérée. 
Mais par ailleurs, la décentralisation a eu des effets sur elles. Au-delà d'une mise en cause, justifiée ou non (cette question n'est pas de notre ressort), du cadre de travail proposé par l'ABF, c'est dans cette perspective qu'il faut notamment comprendre la création de l'ADBDP (1987) et celle plus récente de l'ADBGV (2002) ; leur naissance, leur allant, les sujets dont elles ont jugé bon de se saisir sont significatifs d'un besoin d'échanges entre "pairs", d'une sorte de désir fédératif ou confédératif d'autant plus vifs que Paris se faisait moins entendre.

L'exercice qui est à l'origine de ce texte explique qu'il revête un caractère pédagogique parfois marqué. Tout bien pesé, il n'a pas paru opportun de l'en dépouiller.

Les chiffres donnés, relatifs à l'évolution des aides attribuées par l'État au profit des bibliothèques territoriales, proviennent de la direction du Livre et de la Lecture (DLL) ou du Centre national du livre $(\mathrm{CNL}){ }^{\left({ }^{20}\right)}$. Sauf dans le cas de l'enveloppe du concours particulier, pour lesquels des informations ont été obtenues directement auprès de la direction générale des Collectivités locales au ministère de l'Intérieur (DGCL) ${ }^{211}$. Enfin, cet exposé bénéficie d'échanges avec les conseillers pour le livre et la lecture de plusieurs Directions régionales des affaires culturelles (DRAC) ${ }^{(22)}$. Bien entendu, l'usage fait ici de ces informations et de ces échanges n'engage ni les uns ni les autres.

\subsubsection{Le paysage administratif}

Il y a en France trois grandes catégories de collectivités locales: environ 36000 communes ${ }^{(23)}$ sont réparties dans 99 départements (dont 4 outre-mer) qui sont réparties dans 26 régions (dont 4 outre-mer ${ }^{(24)}$ ). Ces trois catégories de collectivités locales se partagent les compétences qui ne sont pas exercées par l'État. Il n'y a pas entre elles de rapports de subordination. Les communes qui se trouvent sur le territoire d'un département ne sont pas placées dans la dépendance de celui-ci; les départements qui se trouvent dans une région ne sont pas placés dans la dépendance de celle-ci.

Les bibliothèques des collectivités locales comprennent les bibliothèques municipales (BM), qui sont des services municipaux, et les bibliothèques départementales de prêt (BDP), qui sont des services départementaux. Il n'existe pas à l'heure actuelle de bibliothèques relevant des régions. bibliothèque.

Sur 36000 communes, entre 3000 et 4000 sont considérées comme possédant une

Pour l'année 2001, dernière année pour laquelle les statistiques ministérielles ont été publiées, les destinataires de l'enquête statistique annuelle du ministère de la Culture étaient au nombre de 3929.3330 ont répondu.

Sur les 3330 bibliothèques en question, seules 3000 ont été retenues pour l'établissement des statistiques et ont donc été considérées comme des BM dignes de ce nom. Encore les critères ne sont-ils pas d'une extrême exigence : accessibilité à tous les publics; dépenses de personnel égales ou supérieures à 7500 euros (soit le traitement d'un agent de catégorie C à mi-temps) ; dépenses de personnel inférieures à 7500 euros mais budget d'acquisition supérieur à 900 euros et ouverture hebdomadaire d'au moins six heures.

Selon les villes, une BM va d'un local unique à un ensemble comprenant une bibliothèque centrale et plusieurs bibliothèques de quartier.

\footnotetext{
(20) Remerciements, à la DLL, à Christine Bonnefon, Denis Cordazzo, Thierry Grognet, Michel Yvon et surtout François RouyerGayette ; au CNL, à Hélène Coquerand et Laurence Boitard.

(21) François Lucas, directeur général adjoint; Myriam Mahé-Lorent, chef du bureau du financement des transferts de compétence, et son adjoint Sébastien Creusot.

(22) Pascal Allard, Marine Bedel, Martine Blanc-Montmayeur, Nadine Etcheto-Tharel, Elisabeth Meller-Liron, Chantal Valentin, Jean-François Sibers.

(23) 36717 d'après le Bottin des communes et de l'intercommunalité 2004.

(24) Outre-mer, les 4 régions et les 4 départements correspondent aux mêmes aires géographiques. Les régions sont dites monodépartementales.
} 
Pour permettre aux communes de réaliser des économies d'échelle et aux plus petites d'entre elles de bénéficier de services qu'elles ne peuvent faire fonctionner seules (sur 36000 communes, près de 32000 ont moins de 2000 habitants ${ }^{(25)}$ ), elles sont incitées à se regrouper. Dès la fin du XIXème siècle, est créée la formule du syndicat intercommunal à vocation unique (SIVU). Mais c'est surtout depuis la fin des années 1950 que l'État cherche à faire progresser la coopération intercommunale. Depuis une dizaine d'années, diverses lois se sont attachées à hâter le mouvement ainsi qu'à clarifier le paysage.

Ainsi, selon leur taille et en allant des plus petites aux plus grandes, il est désormais loisible aux communes de former des communautés de communes, des communautés d'agglomération ou des communautés urbaines. Elles peuvent aussi s'associer dans le cadre de structures plus souples fondées sur des projets et formalisées par des contrats : "pays" ou "agglomérations".

Au $1^{\text {er }}$ janvier 2004, pas moins de 31424 communes - représentant $82 \%$ de la population participaient à un établissement public de coopération intercommunale $(\mathrm{EPCl})^{(26)}$.

Concernant les communautés de communes, «la construction, l'entretien et le fonctionnement » d'équipements culturels font partie d'un des groupes de compétences optionnels ${ }^{(27)}$. C'est aussi le cas pour les communautés d'agglomération ${ }^{(28)}$ de "la construction, l'aménagement, l'entretien et la gestion" d'équipements culturels. Dans les deux cas, les équipements doivent présenter un "intérêt communautaire". En ce qui concerne les communautés urbaines, "la construction ou l'aménagement, l'entretien, la gestion et l'animation d'équipements, de réseaux d'équipements ou d'établissements culturels" font partie d'un des sept blocs de compétences qu'elles exercent de plein droit ${ }^{299}$. Mais, là encore, il doit s'agir d'équipements "d'intérêt communautaire".

La culture, les équipements culturels constituent un des éléments dont les communes sont le plus réticentes à se dessaisir de la propriété exclusive. En effet, ils sont, aux yeux des villes, les dépositaires privilégiés de leur identité. La notion d'intérêt communautaire est suffisamment souple pour laisser le champ libre, de ce point de vue, à toutes les options, de l'absence totale de transfert d'équipements culturels à la communauté au transfert de tous les équipements.

Pour autant, il ne fait aucun doute que le nombre d'EPCI compétents en matière culturelle est destiné à augmenter. Ils étaient -estime-t-on - $37,5 \%$ à avoir effectué ce choix au $1^{\text {er }}$ janvier 2003.

Toutes les structures intercommunales qui se sont dotées de la compétence culturelle n'ont pas mutualisé leurs BM. Mais des BM en nombre croissant sont, seront bâties d'emblée pour des structures intercommunales ou, existant déjà, sont, seront mutualisées dans le cadre de ces structures ${ }^{(30)}$. Il leur faut adapter leur fonctionnement à cette nouvelle donne.

La mutualisation des $B M$ dans le cadre de structures intercommunales est un phénomène majeur, perçu comme tel mais dont il n'est pas sûr que l'ampleur prévisible et toutes les conséquences aient été pleinement mesurées.

Créées en 1945, les BDP ont vocation à desservir les petites communes ne possédant pas de $\mathrm{BM}$, à les inciter à en créer et à apporter un appui à celles qui en ont une. A l'origine, le plafond de desserte était une population de 15000 habitants. Il a ensuite été remonté à 20000 habitants (1968). Il est de 10000 depuis 1985.

\footnotetext{
(25) Exactement 31948 (recensement général de 1999, site de l'INSEE).

(26) "Bilan de l'intercommunalité, 1 ${ }^{\text {er }}$ janvier 2004", communiqué du ministre délégué aux libertés locales. Disponible sur le site du ministère de l'Intérieur.

(27) Code général des collectivités locales, article L 5214-16.

(28) Ibid., article L 5216-5.

(29) Ibid., article L 5215-20.

(30) Une enquête est actuellement conduite sur ce point par l'ADBGV (voir son site). D'après les statistiques de la DLL, 134 bibliothèques ont déclaré être intercommunales en 2001. Ce chiffre est sujet à caution et, en tout état de cause, sans doute inférieur à la réalité.
} 
Il y a aujourd'hui $98 \mathrm{BDP}{ }^{(31)}$. C'est à la fois plus et moins que de départements proprement dits. Moins : Paris (qui est une commune mais aussi un département) et les trois départements de la "petite couronne" de la région parisienne $(92,93,94)$ n'ont pas de BDP.

Plus: Mayotte, qui est une "collectivité territoriale" dotée d'un conseil général, une "collectivité départementale", possède une BDP (mais pas l'autre collectivité territoriale départementale, Saint-Pierre-et-Miquelon). Territoire d'outre-mer, la Nouvelle-Calédonie en possède une aussi. ${ }^{(32)}$

Le ministère compétent pour les bibliothèques territoriales est le ministère de la Culture. Le ministère de la Culture, c'est, d'une part, une administration centrale située à Paris, en l'occurrence la direction du Livre et de la Lecture (DLL), d'autre part, dans les régions, une administration dite "déconcentrée", les directions régionales des Affaires culturelles (DRAC).

Ce qu'on appelle "les fonctionnaires", la fonction publique, comprend en fait trois grandes "branches" : la fonction publique d'État, la fonction publique territoriale et la fonction publique hospitalière. Les personnels travaillant dans les BM et les BDP sont normalement des fonctionnaires territoriaux. Dans certains cas, il peut aussi s'agir de fonctionnaires d’État.

Selon la définition que l'on adopte, il est possible de faire remonter la décentralisation plus ou moins haut dans le temps. Ce qu'on désigne communément aujourd'hui par l'expression "la décentralisation", c'est un processus législatif initié en 1982-1983 ${ }^{\text {(33) }}$ et poursuivi depuis sur un rythme plus ou moins rapide. Au cours des années 1980, en particulier, sont prises trois mesures majeures : auparavant établissement public, la région devient une collectivité territoriale, à l'instar du département et de la commune ${ }^{(34)}$; pour les décisions prises par la région et le département, le pouvoir exécutif est transféré du préfet respectivement au président du conseil régional (créé en 1986) et au président du conseil général ; le préfet n'exerce plus de contrôle sur les actes des collectivités territoriales qu'a posteriori, et uniquement sur la légalité des actes, non sur leur opportunité.

C'est dans ce processus initié en 1982-1983 que s'inscrivent les mesures relatives aux bibliothèques territoriales dont il sera question dans cet exposé.

Compte tenu de la place disponible, il ne sera pas possible d'y retracer le détail de l'histoire de ces mesures. C'est dommage : comme toujours, les dispositifs en vigueur aujourd'hui résultent d'une interaction entre les intentions du ministère de la culture et l'évolution de la situation, telle que l'ont déterminée au fil des années son action, les circonstances politiques, etc.

Les bibliothèques territoriales ne sont pas les seules à être concernées par la décentralisation. Les bibliothèques scolaires - bibliothèques centres documentaires $(B C D)$ pour les écoles, centres de documentation et d'information (CDI) pour les collèges et les lycées - le sont aussi. Et ce, doublement.

En premier lieu, de la façon suivante. D'une part, la responsabilité de la construction, de l'équipement et du fonctionnement matériel des collèges et des lycées a été transférée, pour les premiers, aux départements, pour les seconds, aux régions ; s'agissant des écoles (élémentaires et primaires), la même responsabilité incombait déjà aux communes depuis le XIXème siècle. D'autre part, les pouvoirs des communes, des départements et des régions se sont accrus.

\footnotetext{
(31) En comptant la bibliothèque Benrheim (Nouvelle-Calédonie), qui joue à la fois le rôle de BM, de BDP et de bibliothèque régionale. L'ADBDP lui fait une place dans ses guides annuels des BDP.

${ }^{(32)}$ Ces autres territoires que sont la Polynésie française et Wallis et Futuna n'ont pas de BDP, sans parler des Terres australes et antarctiques françaises.

(33) Article 1 de la loi $\mathrm{n}^{\circ}$ 82-213 du 2 mars 1982 relative aux droits et libertés des communes, des départements et des régions, article 1 : "Les communes, les départements et les régions s'administrent librement par des conseils élus". Les lois du 7 janvier et du 22 juillet 1983 précisent les modalités du transfert de compétences - dont les compétences culturelles - au profit des collectivités territoriales.

(34) Rappelons que cette mesure figurait déjà dans le projet de loi présenté au référendum le 27 avril 1969 et dont le refus devait entraîner la démission du général de Gaulle de la présidence de la République.
} 
Sous ce double rapport, la décentralisation est susceptible d'avoir eu des effets sur le développement des bibliothèques scolaires.

En second lieu, en transférant les BCP aux départements, en accroissant les pouvoirs des maires et ceux des conseils généraux, la décentralisation est susceptible d'avoir favorisé le rapprochement des $B M$ avec les $B C D$ et, de façon plus neuve, celui des BDP avec les CDI des collèges.

Dans le cas des communes, Bernard Latarjet remarquait au début des années 1990 : "Une municipalité qui fait un effort considérable pour développer son réseau de bibliothèques comprend mal pourquoi il serait étanche au réseau scolaire dont elle a, par ailleurs, la charge [...]" ${ }^{35)}$. Cette observation reste valable. Et pourrait s'appliquer aux conseils généraux.

Notre sujet étant ici les bibliothèques publiques, il ne sera pas question du premier aspect. Et peu du second, qui mériterait une étude particulière, au demeurant difficile à écrire en l'état des informations disponibles.

\subsubsection{L'application de la décentralisation aux bibliothèques territoriales}

\subsubsection{Les bibliothèques municipales}

Les BM dépendent des communes. Mais c'était déjà le cas avant la décentralisation. Elles n’ont donc évidemment pas été "décentralisées". Elles ont malgré tout été concernées par la décentralisation.

Pour comprendre l'attitude qui va être celle du ministère de la Culture, c'est-à-dire en l'occurrence de la DLL ${ }^{(36)}$, lorsque la décentralisation est décidée, il faut se souvenir que la France, du point de vue de la "lecture publique", fait alors figure de retardataire par rapport aux pays qui servent de référence en la matière (pays anglo-saxons et scandinaves). Et que pour la direction - où le service chargé des bibliothèques est constitué de bibliothécaires -, dès lors qu'il est avéré que les villes ne se sont pas saisies spontanément de la question, seule une action déterminée de l'État est à même de sortir la lecture publique de l'impasse.

Des effets positifs d'une telle action, l'évolution de la situation depuis les années 1970 semble de fait attester la réalité. En effet, depuis ces années-là, les constructions de BM se multiplient.

Parce que les élus l'ont voulu, que les politiques culturelles ont le vent en poupe et que le budget des collectivités locales connaît une croissance importante. Certes. Mais aussi parce que les agents du ministère de la Culture ont aidé les bibliothécaires des collectivités territoriales à convaincre les élus de vouloir des bibliothèques. Et parce qu'un budget spécifique - conformément à des préconisations émises dès 1967 - est venu seconder efficacement leurs efforts pédagogiques.

La lecture publique va-t-elle enfin décoller en France? Il y a des raisons de le penser. Encore faut-il - pense la direction du Livre - que l'État poursuive son effort.

Or, dès les mêmes années 1970, se manifeste une atmosphère décentralisatrice qui n'est pas sans faire sentir de premiers effets sur la politique en faveur des bibliothèques. A l'origine des intentions de l'État en la matière ne figure pas seulement, mais figure aussi un certain essoufflement budgétaire.

\footnotetext{
(35) L'aménagement culturel du territoire, rapport, Paris, La Documentation française, 1992, p. 31. "De même", est-il ajouté, " une municipalité qui réclame une antenne universitaire pour fixer sa population étudiante et attirer des entreprises, est fortement tentée de faire jouer à sa bibliothèque de lecture publique un rôle universitaire ».

(36) La direction du Livre est créée en 1975. Elle hérite de la partie lecture publique des compétences de la direction des Bibliothèques et de la Lecture publique, qui existait depuis 1945 et disparaît. C'est en 1982 que la Direction du livre prend le nom de direction du Livre et de la Lecture.

(37) Historique synthétique dans A.-M. Bertrand, Les bibliothèques municipales. Enjeux culturels, sociaux, politiques, Paris, Ed. du Cercle de la librairie, coll. "Bibliothèques", 2002, chap. 1 et 2.
} 
Ainsi, le Vle plan (1971-1975) prévoyait-il le classement de 75 BM, ce qui aurait porté le nombre des BMC de 50 à $125^{(38)}$; il ne s'agissait plus seulement d'assurer la préservation des fonds patrimoniaux d'État, mais de doter le territoire d'équipements qui, dirigés et encadrés par des agents de l'État, c'est-à-dire des agents solidement formés, constitueraient les relais et les acteurs d'une politique cohérente localement et nationalement. Or, sur 75 BM, il n'en sera classé que 4, en 1972 ; et plus aucune après.

En 1980, la réticence de l'État à créer des postes au profit des BMC, jointe à l'interdiction faite aux villes concernées de créer des emplois territoriaux équivalents, aboutit à ce que la part du personnel d'encadrement dans les BMC soit inférieure au taux d'encadrement dans les autres BM ${ }^{(39)}$.

Par ailleurs, c'est en fait dès la fin des années 1970 que sont envisagés, d'une part, le transfert des BCP aux départements, d'autre part, la suppression des subventions spécifiques pour la construction, l'équipement et le fonctionnement des $B M{ }^{(40)}$.

Dans ce contexte, l'action de la direction du Livre en faveur des bibliothèques a tout de la course contre la montre.

Quand, à ce moment là, l'occasion lui est fournie de proposer une politique ${ }^{(41)}$, elle ne l'envisage pas sur le mode du dessaisissement mais, au contraire, comme une amplification de son intervention.

Or, en 1981, un fait politique majeur se produit. Un nouveau président de la République est élu ( $F$. Mitterrand). Et son gouvernement décide de renforcer l'autonomie des collectivités locales en leur transférant des responsabilités, en particulier financières.

Dès lors, la DLL est prise entre deux exigences. D'un côté, il lui faut tenir compte de la volonté politique de décentralisation qui s'impose à tous les ministères. D'un autre côté, elle reste persuadée que, sans une action déterminée de l'État, le développement tant attendu des bibliothèques publiques s'interrompra.

Tout son effort va donc consister à tenter de concilier les deux exigences - l'expression "limiter les dégâts" serait sans doute plus proche de l'état d'esprit des agents de la Direction à l'époque. Le résultat est celui-ci.

En 1980, l'aide apportée par l'État aux BM revêt les formes suivantes.

En fonction des demandes, le ministère de la Culture attribue aux villes des subventions pour la construction et l'équipement de BM ${ }^{(42)}$. Libre de décider du montant des subventions voire de ne pas les attribuer, il se sert de cette latitude pour porter les villes à améliorer leurs projets.

S'agissant du fonctionnement des BM, le ministère :

- rembourse une partie des dépenses des BM aux villes dans lesquelles ces dépenses atteignent un certain niveau (l'objectif est d'encourager les villes à atteindre ce niveau).

- met à la disposition de 54 villes - dotées de BM dites classées (BMC) - les conservateurs qui constituent les directeurs et les cadres de ces BMC ${ }^{(43)}$. Il assure la majeure partie de leur

\footnotetext{
(38) Propositions pour les bibliothèques municipales : de nouvelles responsabilités, un nouveau partenariat des collectivités publiques en faveur du patrimoine et de la lecture. Rapport du groupe de travail sur les bibliothèques municipales classées, présenté à monsieur le directeur du livre et de la lecture, avril 1989, multigraphié, p. 12. Il s'agit du rapport dit Béghain, du nom du président du groupe de travail.

(39) Rapport Béghain, op. cit., p. 14.

(40) Décentralisation et bibliothèques publiques, 1984, multigraphié, p. 15. Il s'agit du "rapport Yvert". Voir aussi le rapport cité ci-dessous, en particulier, pour les BCP, p. 45-47.

${ }^{41)}$ En 1980, un rapport sur les bibliothèques est demandé par le Premier ministre au directeur du Livre (Les bibliothèques en France, dit rapport Vandevoorde, Paris, Dalloz, 1982). Quand le rapport est remis, en juillet 1981, le gouvernement a changé et la décentralisation est décidée. Entrepris dans une autre logique, le rapport s'efforce d'intégrer la nouvelle.

(42) Par construction, on entendra au cours de cet exposé l'ensemble des opérations liées au bâtiment : construction proprement dite, extension, restructuration, etc ; équipement désigne le mobilier et l'équipement informatique.
} 
rémunération (55\%), les villes prenant le reste en charge. Dans la quasi-totalité des cas, le "classement" a pour origine la présence de fonds issus des confiscations effectuées au moment de la Révolution et appartenant à l'État.

- attribue, au cas par cas, des subventions à divers titres, en particulier au profit des fonds patrimoniaux et pour l'achat de livres.

Après la décentralisation, le ministère garde la maîtrise directe de ces subventions attribuées à des titres divers. Mais la situation change sur les autres points.

1) Le ministère continue à mettre des conservateurs d'État à la disposition de 54 communes au bénéfice de leur BMC. Désormais, il assure même intégralement leur rémunération ${ }^{(44)}$.

Mais ces 54 communes sont libres de créer, aux côtés des postes de conservateurs d'État, des postes de conservateurs territoriaux.

2) Est institué le concours particulier.

De quoi s'agit-il ?

Le gouvernement a décidé de rassembler et de fondre une large part des subventions attribuées aux collectivités locales par les divers ministères au sein d'une "dotation générale de décentralisation" (DGC) ${ }^{(45)}$ qui leur sera versée chaque année automatiquement et dont elles pourront disposer comme elles l'entendent.

Du point de vue du ministère de la Culture, cela signifie que les communes pourront éventuellement affecter à d'autres usages que les bibliothèques les subventions qu'il consacrait à favoriser leur construction, leur équipement et leur fonctionnement.

A la demande du ministère est donc identifiée, au sein de la DGC, une partie dite "concours particulier". Les crédits qui s'y trouvent ne sont plus octroyés par l'État selon son bon vouloir, ils reviennent de droit aux villes. Mais, d'une part, elles doivent remplir pour en bénéficier des conditions qui vont dans le sens d'une élévation de la qualité du service. D'autre part, ces crédits ne pourront être utilisés que pour les bibliothèques municipales. ${ }^{(46)}$

Par ailleurs, décidé en 1983, le transfert des crédits du ministère dans la DGC ne s'applique qu'en 1986. Le temps pour le ministère d'achever - en particulier - des opérations de construction.

Le concours particulier s'applique aux BM mais aussi aux bibliothèques intercommunales. L'enveloppe augmente chaque année en fonction de l'augmentation de la dotation globale de fonctionnement (DGF) ${ }^{(47)}$.

Il se subdivise en une "première part" qui, réglementairement, représente $35 \%$ de l'ensemble et une "deuxième part" qui représente $65 \%$.

\footnotetext{
${ }^{(43)}$ Non sans exagération, on qualifie parfois de "loi de nationalisation" la loi qui avait disposé que les conservateurs des BMC seraient des conservateurs d'Etat (1931).

${ }^{44}$ Voir J.-L. Gautier-Gentès, Le contrôle de l'État sur le patrimoine des bibliothèques : aspects législatifs et réglementaires. Essai de présentation critique, $2^{\mathrm{e}}$ éd. corrigée, mise à jour et augmentée, Villeurbanne, ENSSIB, 1999, p. 36-37.

${ }^{(45)}$ A ne pas confondre avec la dotation globale de fonctionnement (DGF). La DGC est censée correspondre aux transferts de compétence.

${ }^{(46)}$ Le concours particulier des communes a été institué par la loi du 7 janvier 1983, modifiée en 1992. Les lois et règlements qui le régissent ont été codifiés dans le Code général des collectivités territoriales. Le texte d'application le plus récent a paru en 1999 (actualisation d'une circulaire de 1993). Il existe deux autres concours particuliers au sein de la DGC : l'un en faveur des ports maritimes de commerce et de pêche (au profit des départements), l'autre pour l'établissement et la mise en œurre des documents d'urbanisme (au profit des communes).

(47) Depuis 1996, la DGF "évolue chaque année en fonction d'un indice égal à la somme du taux prévisionnel d'évolution de la moyenne annuelle du prix de la consommation des ménages (hors tabac) de l'année de versement et de la moitié du taux d'évolution du produit intérieur brut en volume de l'année en cours, sous réserve que celui-ci soit positif" (Code général des collectivités territoriales, article L 1613-1).
} 
La première part prend la suite des crédits remboursant une partie des dépenses de fonctionnement des BM quand le montant atteint un certain niveau. Pour en bénéficier, une commune dont la population est au moins égale à 10000 habitants doit effectuer pour sa BM des dépenses au moins égales à $70 \%$ du montant moyen. Le montant moyen ouvrant droit à remboursement n'est que de $60 \%$ dans le cas des communes dont la population est inférieure à 10000 habitants. particulier.

En 2003 (dépenses de 2001), 1645 communes émargeaient à la première part du concours

La deuxième part prend la suite des crédits consacrés à l'investissement. Elle peut servir à financer la construction, l'extension ou la restructuration de locaux ainsi que l'acquisition de mobilier faisant suite à une de ces opérations; l'achat de logiciels et de matériels informatiques ; l'acquisition de bibliobus ; enfin, les opérations destinées à améliorer les conditions de préservation et de conservation des fonds patrimoniaux.

Chaque région se voit attribuer une enveloppe. Pour qu'une ville en bénéficie, sa $B M$ doit présenter une surface minimale de $0,07 \mathrm{~m}^{2}$ par habitant dans le cas d'une commune comprenant jusqu'à 25000 habitants ; cette surface ne doit en aucun cas être inférieure à $100 \mathrm{~m}^{2}$. Si la commune comprend plus de 25000 habitants, la surface doit être de $0,07 \mathrm{~m}^{2}$ par habitant jusqu'à 25000 et de $0,015 \mathrm{~m}^{2}$ au-delà.

Actuellement, le taux de financement au titre de la deuxième part est en moyenne de $35 \%$.

De 1993 à 2001, a existé une "troisième part" qui a servi à financer à hauteur de 40 \% (taux maximum) la construction et l'équipement de 12 "bibliothèques municipales à vocation régionale" (BMVR) ${ }^{(48)}$. Les conditions étaient les suivantes : commune ou groupement de communes de plus de 100000 habitants ou chef lieu de région; fonds de livres imprimés pour adultes d'au moins 250000 volumes; dans le cas d'une commune ou d'un regroupement de communes n'excédant pas 200000 habitants, au moins $50 \mathrm{~m}^{2}$ par tranche de 1000 habitants; au-delà de 200000 habitants, surface minimale de $10000 \mathrm{~m}^{2}$; présence de plusieurs supports documentaires et des NTIC; projet coopératif.

Il s'agit en fait du même mécanisme que la deuxième part, mais au profit de grosses bibliothèques à qui il a été demandé de jouer un rôle coopératif.

Les BMVR ne sont pas des bibliothèques dépendant des régions en totalité ou en partie mais, là encore, des services entièrement municipaux. "Régionale" signifie que ces bibliothèques ont vocation à accomplir des missions coopératives au-delà des limites de la ou des communes dont elles dépendent. Des régions comportent plusieurs BMVR (et d'autres aucune) ${ }^{(49)}$.

L'évolution de l'enveloppe a été la suivante ${ }^{(50)}$. Comme la $1^{\text {ère }}$ et la $2^{\text {ème }}$ parts, elle a suivi celle de la DGF.

\begin{tabular}{|l|l|}
\hline 1993 & $44685560 \mathrm{~F}$ \\
\hline 1994 & $55065272 \mathrm{~F}$ \\
\hline 1995 & $56001382 \mathrm{~F}$ \\
\hline 1996 & $57989431 \mathrm{~F}$ \\
\hline 1997 & $58778116 \mathrm{~F}$ \\
\hline 1998 & $59591529 \mathrm{~F}$ \\
\hline 1999 & $61247720 \mathrm{~F}$ \\
\hline 2000 & $61750564 \mathrm{~F}$ \\
\hline 2001 & $63865039 \mathrm{~F}$ \\
\hline
\end{tabular}

\footnotetext{
(48) Pour le fonctionnement, les BMVR émargent à la première part du concours particulier.

(49) Il se trouve que les 12 BMVR sont toutes par ailleurs des BMC et bénéficient donc pour fonctionner de conservateurs mis à disposition par l'État.

(50) Source : DGCL.
} 
Depuis la suppression de la troisième part en 2002, les crédits annuels qu'elle comprenait c'est-à-dire la part de l'enveloppe qui n'était pas constituée de reliquats de la deuxième part (voir plus bas) - sont venus abonder la première et la deuxième parts. En liaison avec le ministère de l'Intérieur et le comité des finances locales, le ministère de la Culture est à la recherche d'une voie qui lui permettrait de continuer à faciliter par une aide financière spécifique et avantageuse la construction d'équipements importants.

\subsubsection{Les bibliothèques centrales de prêt}

S'agissant des BDP, le terme de décentralisation s'applique pleinement au processus conduit dans les années 1980.

En effet, les bibliothèques centrales de prêts (BCP), services de l'État dans les départements, gérées directement par l'administration centrale jusqu'en 1982 puis placées sous l'autorité des préfets (appelés à ce moment là commissaires de la République), sont alors transférées aux départements. C'est en $1992{ }^{{ }^{(51)}}$ qu'elles prennent officiellement le nom de bibliothèques départementales de prêt (BDP).

Le transfert a concerné l'ensemble des services : bâtiments, matériels, collections, emplois.

Comme il l'a fait pour les BM, le ministère de la Culture, tandis que l'État se dessaisissait des BDP, a voulu donner toutes ses chances à ce mode de desserte :

- Décidé en 1983, le transfert ne devient effectif qu'au $1^{\text {er }}$ janvier 1986. En 1986, l'État s'engage par ailleurs à mener à bien dans les 4 ans, à compter du transfert, des constructions ou des agrandissements ; en 1990, le terme sera reporté au 31 décembre 1991. Il s'agissait, d'une part, de doter d'une BCP les départements qui n'en possédaient pas encore (17 d'après le rapport Vandevoorde, 1982); d'autre part, de mettre des bâtiments à niveau ${ }^{(52)}$. En fait, les travaux se poursuivront jusque dans la seconde moitié des années $1990{ }^{153)}$.

Si l'État n'avait pas décidé de doter lui-même d'une BDP les départements qui n'en possédaient pas, tous ces départements en auraient-ils créé une ? Nous ne le saurons jamais.

Probablement pas. Faut-il considérer à cet égard comme probant le fait que le territoire de Polynésie française n'en possède toujours pas? D'un côté, il s'agit d'une collectivité à maints égards très particulière, difficile à desservir parce que constituée d'un ensemble d'îles dont le périmètre équivaut à celui de l'Europe. D'un autre côté, précisément du fait de cette configuration, une BDP s'imposerait.

- Pour mener à bien ce programme, le ministère de la Culture a conservé la disposition des crédits afférents.

En 1992, la moitié de ce budget, soit 31 MF sur 62, augmentée de crédits non dépensés au fil des années au titre de la deuxième part $(21 \mathrm{MF}){ }^{(54)}$ et de ponctions sur le montant des première et deuxième parts, sert à constituer la troisième part du concours particulier, celle qui sert à financer le programme de BMVR. ${ }^{(5)}$

A la même date, l'autre moitié est intégrée dans la DGC versée aux départements. Mais, à l'exemple du dispositif institué pour les BM (deuxième part du concours particulier), elle y forme un concours particulier pour l'investissement. Les dépenses prises en compte pour le remboursement

\footnotetext{
(51) Loi du 13 juillet.

${ }^{(52)}$ Ainsi que construire une annexe (Bouches-du-Rhône). Source : décret $n^{\circ}$ 86-278 du 26 février 1986.

(53) Pour les travaux restant à réaliser au 31 décembre 1991 : B. Calenge, article cité, p. 25. En novembre 1995, le directeur du livre et de la lecture signale comme restant à réaliser pour achever le programme de 33 constructions les opérations de Corse et des Yvelines, des Bouches-du-Rhône (annexe) et de Mayotte (Le livre en campagne, bibliothèques départementales en jeu, actes du colloque du cinquantenaire, ADBDP, 1996, p. 11). C'est en 1997 que la BDP de Mayotte est mise en service. (54) Il s'agit plus précisément de "la moitié des crédits non dépensés au titre de l'exercice 1991" (Code général des collectivités territoriales, article R 1614-75).

(55) CSB, Rapport du président pour l'année 1992, Paris, association du CSB, 1993, p. 55-57.
} 
partiel versé aux départements sont, d'une part, celles effectuées pour les BDP elles-mêmes, d'autre part, celles effectuées pour les bibliothèques des communes qu'elles desservent.

Il n'a pas été créé au profit des BDP de concours particulier pour le fonctionnement ; les crédits gérés à ce titre par le ministère de la culture ont été fondus dans la DGC ${ }^{(56)}$, y compris ceux correspondant à la rémunération des agents sur emplois d'État.

- Services départementaux, les BDP ont désormais vocation à employer normalement des agents territoriaux. Avant le transfert, l'État encourage les départements à créer des emplois en leur attribuant à titre temporaire des subventions représentant une partie de la rémunération. En 1984, sont dénombrés 132 agents des départements (et 72 relevant d'associations) pour 924 agents de l'État ${ }^{157}$. Le 31 décembre 1986, soit un an après le transfert, les personnels territoriaux représentent $35 \%$ des effectifs ${ }^{(58)}$.

Au terme d'un processus qui ne s'achèvera qu'en 1997, et supposait la parution de statuts établissant une homologie entre la filière de l'État et la filière territoriale (1991-1992), les personnels d'État

- soit auront quitté les BDP pour rejoindre une bibliothèque de l'État ;

- soit auront choisi de devenir fonctionnaires territoriaux ;

- soit seront restés fonctionnaires de l'État mais en étant rémunérés par le département (procédure du "détachement").

Par ailleurs, en 1985, à quelques mois du transfert, le directeur du Livre et de la Lecture adresse aux directeurs des BCP une circulaire qui, tout en prenant acte du fait qu'il reviendra bientôt aux départements de fixer leurs missions, fait état des orientations qu'il lui paraît souhaitable d'adopter ${ }^{(59)}$. Il s'agit d'aider les directeurs vis-à-vis de leurs nouveaux "patrons" en leur fournissant un texte de référence. Celui-ci réitère en particulier la recommandation de se consacrer surtout aux communes de moins de 10000 habitants. Le propos est à la fois de prendre acte du développement des BM dans les communes de plus de 10000 habitants, et de l'encourager. Ou plus précisément de ne pas y faire obstacle en dispensant les villes concernées de se doter d'une BM.

\subsubsection{Le rôle de l'État vu par lui-même}

\subsubsection{Les mesures prises}

En 1983-1985, le transfert dans la DGC des crédits d'investissement et de l'essentiel des crédits de fonctionnement gérés directement par le ministère de la Culture est donc programmé. En matière de bibliothèques mais aussi plus généralement, les communes et les départements, pour décider de leur politique, dépendront moins de l’État.

C'est dans ce contexte que le ministère de la Culture envisage de prendre un certain nombre de mesures. Le principal document de référence en la matière est un rapport qui, demandé par le directeur et de la lecture en octobre 1983, est élaboré par un groupe de travail présidé par un inspecteur général des bibliothèques et qui lui est remis au début de $1984{ }^{(60)}$.

Selon les cas, il s'agit de parer aux risques dont la décentralisation paraît porteuse. Dans d'autres cas, la décentralisation et les risques dont elle est réputée porteuse font plutôt figure d'occasions saisies pour prendre des dispositions jugées utiles de toute manière, la décentralisation ne faisant que les rendre encore plus utiles.

\footnotetext{
(56) Les crédits représentant l'aide de l'État au fonctionnement des BDP dans la DGC étaient estimés à 490 MF en 2001 (site du ministère de la Culture, nov. 2003).

(57) Rapport Yvert, op. cit., p. 9.

${ }^{(58)}$ A. Caraco, "Dix ans après. L'évolution des bibliothèques départementales depuis la décentralisation", BBF, t. 42, 1997, $\mathrm{n}^{\circ}$ 5 , p. 16.

${ }^{(59)}$ Circulaire DLL n ${ }^{\circ} 85-47$ du $1^{\text {er }}$ août 1985.

${ }^{(60)}$ Il s'agit du rapport dit Yvert, du nom de l'inspecteur général des bibliothèques président du groupe de travail (op. cit.).
} 
Parmi les mesures prises, citons :

- La publication de statuts assurant aux personnels territoriaux le même niveau de qualification qu'aux personnels d'État (1991). En particulier dans le cas du personnel d'encadrement (conservateurs et bibliothécaires), formé, par ailleurs, dans la même école que ses collègues de l'État (ENSSIB).

- La nomination dans les DRAC de "conseillers pour le Livre et la Lecture". C'est surtout eux qui, désormais, se substituant dans ce rôle aux agents de l'administration centrale (bureaux, inspecteurs généraux des bibliothèques), auront pour tâche de plaider pour les bibliothèques auprès des villes et des départements, de les renseigner sur les aides de l'État, d'accompagner leur démarche.

On ne comprendra pas bien la nature des relations qu'entretiennent actuellement l'État et les collectivités territoriales du point de vue de la politique culturelle si l'on n'a pas souligné le rôle croissant joué par les DRAC. En effet, au cours des dernières années, la décentralisation culturelle s'est accompagnée d'un mouvement de déconcentration. Aux DRAC, l'administration centrale du ministère de la culture a délégué une part croissante de son budget. A charge pour elles de le répartir, en fonction d'orientations données par Paris. Un des modes de répartition est la signature de contrats, en particulier avec les villes.

Comme tous les équipements culturels, les bibliothèques territoriales sont concernées par ce mouvement de déconcentration administrative et budgétaire.

- La création d'agences régionales de coopération entre bibliothèques. Au fil des années, celles-ci allaient tendre à se transformer en structures compétentes à la fois pour les bibliothèques et pour le livre (aide à la publication, etc.). Il faut y ajouter une structure nationale, la Fédération française de coopération entre bibliothèques (61). Créée en 1985, celle-ci ne fédère pas véritablement les agences régionales; mais conduit, en coopération ou non avec elles, des actions en faveur du patrimoine (mois du patrimoine écrit) ou du développement de la lecture (la lecture dans les prisons, dans les hôpitaux, etc.).

- Des dispositions réglementaires renforçant le droit de regard de l’État sur le fonctionnement des BM et des BDP $\left(1988^{(22)}\right)$.

La nature du contrôle exercé par l'Inspection générale des bibliothèques est précisée. II porte sur tous les aspects du fonctionnement.

L'envoi chaque année d'un rapport d'activité au ministère de la Culture, sous la forme de réponses à un questionnaire qui leur est adressé - cet envoi devient une obligation. L'exploitation de ces questionnaires donne lieu chaque année à la publication de statistiques nationales. Elles permettent de mesurer les évolutions favorables et défavorables : baisse ou augmentation de la proportion de personnel qualifié, progression du nombre de bibliothèques offrant des accès à Internet, etc. Elles permettent aussi aux villes de se situer les unes par rapport aux autres. Au même titre que les recommandations de l'État, elles sont utilisées par celui-ci et par les professionnels pour inciter les collectivités territoriales à tirer leur BM ou leur BDP vers le haut.

Un conseil national scientifique du patrimoine des bibliothèques publiques est créé en 1989. Les communes sont tenues de lui soumettre en particulier tout projet de restauration d'un document patrimonial.

De cette liste, se dégage l'idée que l'État se fait désormais de son rôle : incitation (action pédagogique en faveur du développement des bibliothèques, aides financières); conseil (construction et aménagement des BM; mise en place, dans les départements, de plans de

\footnotetext{
(61) Dénomination actuelle : Fédération française pour la coopération des bibliothèques, des métiers du livre et de la documentation.

${ }^{(62)}$ Il s'agit du décret de 9 novembre. La teneur en a été intégrée depuis dans le Code général des collectivités territoriales, partie réglementaire.
} 
développement de la lecture, etc.) ; préservation et mise en valeur du patrimoine; développement de la coopération.

La politique menée depuis les années 1990 confirme l'existence de cette doctrine de fait. On va voir en particulier l'État :

s'agissant de l'incitation,

- agir dans une perspective de démocratisation culturelle en incitant financièrement les communes à recruter des "médiateurs du livre" (à partir de 1996) ;

- œuvrer en faveur de l'égalité devant l'offre documentaire en incitant financièrement les communes à créer des "relais livre en campagne" (à partir de 1997), structures intercommunales et polyvalentes (il ne s'agit pas seulement de bibliothèques) puis des "espaces culture multimédia" (à partir de 1998) et en équipant les petites BM et les relais livre en campagne de matériel multimédia (1998-1999).

\section{s'agissant du conseil,}

- publier un ouvrage permettant de concevoir une bibliothèque en fonction de la population à desservir (Bibliothèques dans la cité. Guide technique et réglementaire, 1996),

\section{s'agissant des fonds patrimoniaux,}

- en ce qui concerne le signalement, poursuivre la publication, lancée dès avant la décentralisation, des catalogues régionaux d'incunables et entreprendre celle de catalogues régionaux des fonds musicaux (depuis 1989) ${ }^{(63)}$; piloter avec le concours de la BnF et contribuer à alimenter un Répertoire des manuscrits littéraires du XX⿳亠丷⿵冂⿱十口犬 siècle (depuis 1995).

Reprise à partir de 1980, la publication du Catalogue général des manuscrits des bibliothèques publiques de France, elle, est interrompue depuis une quinzaine d'années (le dernier volume paru, le tome 65 , a été publié en 1990$)^{(64)}$. Mais la décision a été récemment prise conjointement par le ministère de la Culture et celui de l'Éducation - de lui substituer un catalogue national informatisé, dans lequel sera versé le Catalogue général après rétroconversion et qui comprendra le catalogue des manuscrits de la BnF.

- en ce qui concerne la conservation, diffuser des recommandations (Protection et mise en valeur du patrimoine des bibliothèques. Recommandations techniques, 1998) ; étendre le champ d'application de la deuxième part du concours particulier en matière de fonds patrimoniaux (1999 $\left.{ }^{(65)}\right)$, etc.

- en ce qui concerne la valorisation, financer fortement, depuis le début des années 1990, l'organisation sous l'égide de la FFCB du "Mois du patrimoine écrit", manifestation annuelle comportant des expositions locales et un colloque où se retrouvent, autour des collections patrimoniales, des bibliothécaires et des chercheurs ${ }^{(66)}$; faire publier avec le concours d'un établissement bancaire un répertoire des $B M$ - territoriales ou non - qui possèdent des fonds patrimoniaux (Patrimoine des bibliothèques de France. Un guide des régions, 1995) ainsi que l'ouvrage Le Moyen Âge en lumière, d'après l'iconothèque de l'IRHT (2002) (67).

Cofinancé par la DLL, le microfilmage par l'Institut de recherche et d'histoire des textes du CNRS des manuscrits médiévaux des BM, lancé dès la fin des années 1970, relève à la fois de la préservation et de la valorisation (entre les deux, la frontière est souvent ténue). Il en est de même de l'inscription dans des contrats de plan État-Région 2000-2006 de la constitution de bases régionales, alimentées par des opérations de numérisation (Champagne-Ardenne, Aquitaine).

\footnotetext{
${ }^{(63)}$ Opération copilotée par la BnF et la direction de la Musique, de la Danse, du Théâtre et des Spectacles. Elle inclut mais dépasse les fonds des BM.

(64) Sans compter un tome 66, imprimé en 1993 et non diffusé, et l'index général publié également en 1993.

(65) Circulaire INTB9900046 C du 11 mars 1999. Les fonds concernés ne sont plus seulement les fonds "anciens" mais les fonds "anciens, rares et précieux" ; la numérisation est ajoutée à la liste des opérations favorisées.

${ }^{(66)} \mathrm{D}$ 'abord simple journée organisée par certaines agences de coopération (1988), cette manifestation devient un "mois" et s'accompagne d'un colloque à partir de 1990 ; la FFCB joue un rôle déterminant dans l'organisation de celui-ci à partir de 1991, l'aide financière de la DLL s'accroît considérablement en 1992.

(67) "Le Moyen Âge en lumière", c'est aussi un site, un DVD et un cédérom scolaire.
} 
s'agissant de la coopération et des réseaux :

- organiser, autour de la $\mathrm{BnF}$, un réseau de bibliothèques dont des $\mathrm{BM}$ (attribution de moyens nouveaux pour collecter et conserver le dépôt légal, attribution de crédits pour cultiver des spécialités documentaires complétant les fonds de la BnF, rétroconversion des catalogues de fonds patrimoniaux pour versement dans le Catalogue collectif de France ${ }^{(68)}$ ).

- porter la Bpi à rendre des services accrus aux BM et aux BDP (pilotage d'un consortium pour l'accès à des documents électroniques en ligne, etc.)

Par ailleurs, le ministère de la Culture attribue aux bibliothèques territoriales des crédits d'achat de livres à l'occasion d'une construction, ou de la constitution de fonds autour d'un domaine ou d'un thème ${ }^{(69)}$; il s'agit d'une diversification des aides attribuées en la matière avant l'institution du concours particulier. En outre, le ministère acquiert au profit des BM des droits sur des films documentaires, etc.

Autant que d'une aide aux bibliothèques, ces mesures relèvent du soutien aux auteurs, aux libraires et aux éditeurs ; mais elles profitent aux bibliothèques.

A l'histoire, que nous appelons de nos vœux, de la politique de l'État à l'égard des bibliothèques territoriales depuis 25 ans, il appartiendrait de déterminer si des domaines et des actions ont été privilégiés par rapport à d'autres, et pourquoi.

S'agissant des fonds patrimoniaux, il serait sans doute possible de mettre en relief que l'État, s'il n'a jamais douté de ses responsabilités en la matière, en a déduit des actions plus ou moins conséquentes. Dans les milieux professionnels, depuis trente ans, le climat n'est guère favorable au patrimoine, même si la généralisation de cette notion a offert une chance aux collections concernées d'échapper à l'obsolescence dans laquelle semblait l'enfermer la notion antérieure de "fonds anciens". A ce climat, l'État n'aura pas manqué de se montrer plus ou moins perméable, plus ou moins imperméable selon les gouvernements et les moments.

Par ailleurs, la valorisation et la conservation semblent bien avoir fait l'objet de plus d'attention de sa part que le traitement (inventaire et catalogage) - et ce alors, d'une part, qu'il s'agit en France d'un vaste chantier, et d'autre part, qu'inventaire et catalogage constituent un préalable à l'exploitation des fonds et contribuent à leur préservation. (Bien entendu, la valorisation ne saurait être opposée au traitement, pas plus qu'à la conservation; elle est susceptible de concourir à sensibiliser le public et les autorités concernées à la nécessité d'assurer le traitement et la conservation).

Les lacunes constatées en matière de traitement des fonds patrimoniaux mériteraient une étude particulière. Elles tiennent à de nombreuses raisons, parmi lesquelles la priorité donnée à la lecture publique dans le discours professionnel et un déficit de compétences, ces deux facteurs tendant d'ailleurs à se renforcer l'un l'autre.

Leur persistance n'en peut pas moins passer pour significative du dilemme posé à l'État en matière d'intervention auprès des collectivités locales.

En effet, d'un côté, il est permis de penser qu'il appartient à chaque bibliothèque d'élaborer un catalogue complet de ses collections. Et que l'État n'a donc pas à y prêter la main. D'un autre côté, ce travail, les bibliothèques peinent manifestement en l'occurrence à l'accomplir. Dès lors, l'État ne doit-il pas intervenir? Et si oui, sous quelle forme? A supposer qu'il soit bien disposé à cet égard, a-t-il les moyens de satisfaire aux besoins, veut-il/peut-il se les donner ? Car la tâche est lourde à plus d'un titre (nombre des documents, dispersion des fonds à traiter, niveau et variété des compétences nécessaires).

\footnotetext{
${ }^{(68)}$ Ce catalogue fédère le catalogue de la $\mathrm{BnF}$ et celui des bibliothèques de l'enseignement supérieur ; y sont aussi signalés les fonds patrimoniaux de certaines BM (48 en 2001, hors Paris).

(69) Ces deux types de crédits proviennent du CNL, établissement public présidé par le directeur du Livre et de la Lecture et qui, avec la Direction, constitue une des deux administrations dont il dispose directement.
} 
Dilemme classique. Il est ici d'autant plus tendu - et aussi d'autant plus visible - qu'il concerne un domaine dans lequel l'État se reconnaît des devoirs propres.

\subsubsection{Les mesures qui n'ont pas été prises}

Pour confirmer l'analyse esquissée dans les pages qui précèdent et approcher l'idée que l'État se fait désormais de son rôle, deux séries d'éléments ne revêtent pas moins d'intérêt que la liste des mesures prises : les mesures qui n'ont pas été prises et celles qui ont fait l'objet d'une doctrine fluctuante.

jour :

C'est ainsi que les dispositions suivantes, préconisées dans le rapport Yvert, n'ont pas vu le

- Promulgation d'une loi sur les bibliothèques (un serpent de mer en France) fixant des obligations aux collectivités territoriales en la matière. Il convient de noter qu'il est loisible à une ville de ne pas posséder de BM. Et que l'existence de statuts pour les personnels de bibliothèques ne vaut pas obligation pour les villes d'employer de tels personnels.

En 1989, le rapport Béghain reprend la proposition du rapport Yvert - qui elle-même n'était pas inédite - d'une loi sur les bibliothèques. Sans plus de succès.

Depuis, l'idée d'une loi sur les bibliothèques refait régulièrement surface. Plusieurs fois, au cours de la seconde moitié des années 1990 , le ministère de la Culture a fait savoir qu'il la mettait à chantier, que son élaboration progressait, qu'elle était presque prête. On l'attend toujours. concernés.

Il y a en fait à ce sujet deux écoles dans les milieux administratifs et professionnels

Ceux qui pensent que laisser la création et le fonctionnement des bibliothèques à leur seule discrétion des collectivités territoriales c'est ne pas assurer l'égalité des citoyens devant l'offre et courir le risque de déprofessionnalisation (auquel s'ajoute maintenant, dans les discours, celui de la privatisation).

Ceux qui constatent que globalement, du point de vue des bibliothèques, les villes et les départements n'ont pas démérité et qu'il y a lieu de leur faire confiance ; ne le voudrait-on, il le faudrait bien, car les collectivités territoriales ne sont pas disposées à se laisser imposer des obligations, surtout sans ressources supplémentaires. ${ }^{(70)}$

Au cours des années 1980, les milieux professionnels étaient unanimes à demander une loi sur les bibliothèques. En apparence, c'est toujours le cas; la loi fait régulièrement partie des revendications présentées aux pouvoirs publics par l'ABF. En fait, non moins régulièrement, des directeurs font savoir qu'à leur avis, quoi qu'on en pense, l'heure de la loi sur les bibliothèques est passée; voire, émettent des doutes quant à son opportunité compte tenu de l'évolution du contexte.

Ces réserves émanant des milieux professionnels eux-mêmes constituent un phénomène nouveau, et particulièrement significatif de l'évolution des mentalités quant aux responsabilités et/ou aux possibilités de l'État en matière de lecture publique.

Quand l'idée d'une loi a réémergé, rue de Valois et dans une large part des milieux professionnels, autour de 1995, c'était malheureusement dans une large mesure à la suite des déboires des BM avec des municipalités relevant du Front national et dans la perspective de "garantir le pluralisme". Ce qui était le meilleur moyen de condamner la loi à ne jamais voir le jour. Car on s'est vite rendu compte, dans les milieux professionnels, que garantir le pluralisme risquait en fait d'obliger les BM à assurer la représentation des idées de l'extrême droite, ce dont les bibliothécaires ou du moins de nombreux bibliothécaires n'avaient pas envie. Devenue "loi sur le pluralisme", la loi a été récusée jusque dans la partie des milieux professionnels qui attendait une "loi sur les bibliothèques"; c'était jeter le bébé avec l'eau du bain, oublier qu'une loi sur les bibliothèques pouvait avoir, devait avoir d'autres objectifs que de garantir le pluralisme.

\footnotetext{
(70) Sur ces deux écoles, voir, dans les actes du colloque du cinquantenaire des BDP en nov. 1995 (op. cit., p. 30-31, 81-82), les très significatifs dialogues entre Christine Bonnefon (Syndicat national des bibliothèques) et Jean-Sébastien Dupuit (directeur du Livre et de la Lecture) d'une part et entre Marie-Claire Millet (direction du Livre et de la Lecture) et Pascal Ory (historien des politiques culturelles et maire-adjoint en charge de la culture) d'autre part. Pour la loi : C. Bonnefon, appuyée par M.-C. Millet. Contre: P. Ory ("le volontarisme législatif a quand même du plomb dans l'aile"). J.-S. Dupuit adopte la position médiane dont il est question plus bas (p. 30-31 mais aussi 178-179).
} 
Entre une loi très contraignante et aucune loi, une position médiane se dégage parfois. C'est celle vers laquelle a paru s'orienter la DLL au cours de la seconde moitié des années 1990 - la DLL qui, ayant reçu la consigne politique d'élaborer une loi, était prise entre ce qu'on a nommé les deux écoles et était vouée, de ce fait, à rechercher une solution conciliatrice. Ainsi, dans les préprojets élaborés à ce moment là, l'obligation pour chaque ville de disposer d'une bibliothèque n'est-elle pas validée ; il est en revanche fait obligation à celles qui en possèdent une bibliothèque de recourir à des professionnels.

- Publication d'un "Code des prescriptions et procédures techniques" sur lequel auraient pu s'adosser l'évaluation et le contrôle.

- Création d'un "Conseil national des bibliothèques publiques". Lui sera préféré, doté à la fois d'un champ plus large et d'un pouvoir d'intervention plus limité, un Conseil supérieur des bibliothèques (1989).

C'est bien dans le registre du conseil que l'État entend désormais se tenir, par opposition à celui de la prescription.

Il se trouve qu'un domaine se trouve au confluent du conseil et de la prescription. On veut parler du contrôle. Or, justement, il a fait l'objet d'une conception hésitante. Selon les époques et les plumes, l'existence d'une Inspection générale des bibliothèques est jugée indispensable ou inopportune. Et en fait, la doctrine de l'inopportunité l'emportera jusqu'en 1996, date à laquelle l’Inspection est reconstituée après une période de dépérissement.

Le contrôle semble faire aujourd'hui l'objet du consensus suivant :

- le contrôle est justifié comme tel dans un certain nombre de cas, au premier rang desquels la situation des fonds patrimoniaux ;

- à ces réserves près, il faut plutôt entendre par contrôle : expertise, évaluation, conseil ; il est significatif de ce point de vue qu'aucune ville n'est obligée de tenir compte des recommandations de l'Inspection générale des bibliothèques.

Il est à noter que, ainsi défini, le contrôle exercé par l'État est non seulement bien toléré par les collectivités locales mais souvent demandé.

C'est aussi vers l'Inspection générale des bibliothèques qu'elles se tournent parfois, de plus en plus souvent semble-t-il, pour intervenir à titre d'expert et d'arbitre dans des différends : entre un directeur et l'hôtel de ville, entre un directeur et ses collaborateurs, etc.

Doivent être relevés par ailleurs le dépérissement, depuis plusieurs années, tant du Conseil supérieur des bibliothèques ${ }^{711}$ que du conseil national scientifique du patrimoine des bibliothèques publiques. Le cas du premier dépasse la question du degré de contrainte que l'État croit devoir ou pouvoir imposer aux collectivités locales ; mais il n'est pas non plus tout à fait étranger à ce sujet.

\subsubsection{Une curiosité}

Pour mémoire, doit être mentionnée une disposition qui, ailleurs en Europe, apparaîtra probablement comme typique du jacobinisme français. L'État fixe le nombre maximal de conservateurs territoriaux que chaque BM et chaque BDP sont autorisées à employer, en fonction de la population à desservir. ${ }^{(72)}$

Dans une optique de développement des bibliothèques, cette mesure paraît contreproductive. Plus nombreux sont les personnels qualifiés employés par les bibliothèques, plus la qualité des prestations est susceptible de s'élever.

\footnotetext{
(71) Quant à l'avenir du CSB, des propositions ont été demandées à son nouveau secrétaire général (2003).

(72) Dernier arrêté en date : arrêté du 19 décembre 2000, modifié par l'arrêté du 2 mai 2002 (Journal officiel du 5).
} 
Cette mesure ne se comprend que si l'on sait qu'elle a pour origine non le ministère de la Culture mais le ministère de l'Intérieur, chargé des collectivités territoriales. Un ministère de l'Intérieur toujours soucieux que les villes ne vivent pas au-dessus de leurs moyens et qu'un déséquilibre ne se crée pas entre les villes.

En l'occurrence, la crainte que les villes, emportées par une passion pour la lecture publique, ne créent plus d'emplois de conservateurs qu'il n'était raisonnable - cette crainte était malheureusement vaine : il y a un écart marqué entre le nombre de postes autorisés et le nombre de postes effectif. Et il est piquant de constater que cette disposition est parfois utilisée à rebours des principes qui ont conduit à l'édicter : l'Inspection générale des bibliothèques - pour ne citer qu'elle - s'en sert pour convaincre les villes et les départements de créer tous les postes autorisés, transformant en quelque sorte la permission en recommandation.

Cette disposition devrait être prochainement supprimée.

\subsubsection{Quel bilan?}

Quel bilan peut-on tirer de l'application de la décentralisation aux bibliothèques territoriales ? De la politique conduite, depuis, par les villes et les départements ?

Si surprenant que cela puisse paraître, un tel bilan n'existe pas. Ne sont disponibles que des éléments de bilan. En outre, ils ne sont pas toujours exempts d'a priori.

Au demeurant, l'exercice n'est pas sans présenter des difficultés. Par rapport à quoi évaluer la politique conduite par les collectivités territoriales ? Par rapport à ce qu'aurait fait l'État? Mais qui peut jurer de ce qu'il aurait fait?

\subsubsection{Les bibliothèques municipales}

\section{a) L'investissement}

Ainsi qu'il a déjà été précisé, il y a aujourd'hui près de 3000 BM en France. Elles étaient trois fois moins nombreuses en 1980. La surface totale des BM est passée de $679136 \mathrm{~m}^{2}$ en 1980 à 2045044 en 2001. ${ }^{(73)}$ Certains des bâtiments de référence de l'architecture contemporaine sont des BM.

Le nombre des usagers inscrits est passé de 2609000 en 1980 - soit $10 \%$ de la population desservie - à 6744181 en 2001, soit $17,4 \%$ de la population desservie ${ }^{(74)}$. Aux usagers inscrits s'ajoutent des usagers non inscrits dont on estime que la moyenne se situe autour de $7 \%$.

Il est d'usage de créditer le concours particulier de cet incontestable développement. Qu'en est-il exactement de ce point de vue ? La réponse à cette question dépend du référent dont on se dote.

Premier référent possible : la situation qui aurait consisté à fondre les crédits du concours particulier dans la DGC.

D'un côté, les BM commencent à se développer en France dans les années 1970. Et si elles se développent, c'est parce que les élus le veulent. Il est permis de penser que des BM auraient vu le jour même en l'absence d'aides spécifiques de l'État.

D'un autre côté, si la volonté des élus se traduit dans les faits d'une façon aussi marquée, c'est parce qu'à leur intention de construire une BM, spontanée ou suscitée, répond l'attribution par l'Etat de crédits spécifiques.

\footnotetext{
(73) Chiffres arrondis. Source : ministère de la Culture et de la Communication, DLL, Bibliothèques municipales [...] Données 2001, Paris, DLL, 2003, p. 212-213.

(74) Ibid.
} 
C'est à ce besoin que répond le concours particulier. Mais les crédits gérés directement par le ministère de la Culture avant l'institution du concours particulier y répondaient aussi.

De ce point de vue, ce dont il faut créditer le concours particulier, entendu comme la "sanctuarisation" dans la DGC des crédits destinés aux bibliothèques, ce n'est pas d'avoir permis le développement des BM. Mais plutôt d'avoir fait en sorte qu'il ne s'interrompe pas.

Deuxième référent possible : le dispositif en vigueur avant la décentralisation, c'est-à-dire la gestion directe par l'administration centrale du ministère de la culture des budgets d'aide à la construction et à l'équipement.

Jugé à cette aune, le dispositif du concours particulier paraît bien devoir faire l'objet d'une appréciation positive, à la condition qu'elle n'exclue ni les nuances ni les questions.

\section{Bilan quantitatif, bilan qualitatif}

De 1986 à 2002, le concours particulier a contribué à financer les opérations suivantes ${ }^{(75)}$.

\begin{tabular}{|c|c|c|c|c|c|c|}
\hline & Bâtiments & Équipement & Informatique & Patrimoine & Bibliobus & Totaux \\
\hline 1986 & 74 & 41 & 21 & 8 & 1 & 145 \\
\hline 1987 & 127 & 60 & 29 & 13 & 2 & 231 \\
\hline 1988 & 152 & 86 & 63 & 20 & 3 & 324 \\
\hline 1989 & 163 & 92 & 74 & 15 & 4 & 348 \\
\hline 1990 & 178 & 89 & 101 & 13 & 5 & 386 \\
\hline 1991 & 258 & 112 & 100 & 5 & 2 & 477 \\
\hline 1992 & 218 & 107 & 83 & 8 & 2 & 418 \\
\hline 1993 & 241 & 135 & 104 & 9 & 2 & 491 \\
\hline 1994 & 235 & 151 & 117 & 16 & 1 & 520 \\
\hline 1995 & 203 & 150 & 116 & 12 & 8 & 489 \\
\hline 1996 & 214 & 131 & 191 & 13 & 6 & 555 \\
\hline 1997 & 232 & 168 & 237 & 8 & 3 & 648 \\
\hline 1998 & 276 & 135 & 286 & 5 & 3 & 705 \\
\hline 1999 & 302 & 152 & 363 & 1 & 0 & 818 \\
\hline 2000 & 284 & 165 & 329 & 5 & 2 & 785 \\
\hline 2001 & 231 & 166 & 238 & 2 & 6 & 643 \\
\hline 2002 & 234 & 140 & 263 & 8 & 5 & 650 \\
\hline Totaux & 3622 & 2080 & 2715 & 161 & 55 & 8633 \\
\hline
\end{tabular}

Il est certain que sans l'aide financière apportée par l'État, une certaine proportion de ces opérations, sinon toutes, n'aurait pas été conduite.

D’autres auraient été conduites, mais auraient présenté un moindre degré de qualité. En effet, l'obtention de cette aide supposait, elle suppose que soient remplies des conditions, et d'abord en matière de surfaces.

Cependant, le bilan n'aurait-il pas été le même si l'administration centrale du ministère de la Culture avait conservé la libre disposition des aides attribuées? Le concours particulier s'est-il avéré être un dispositif particulièrement attractif, et si oui, en quoi ?

\section{$>$ Le cadre et les conditions de l'attribution}

En transformant les subventions octroyées par l'État selon son bon vouloir en crédits appartenant de droit aux communes, pour peu qu'elles remplissent certaines conditions, le concours particulier n'a-t-il pas eu, sur la décision de construire, un effet positif ? C'est possible.

Toutefois, l'expérience montre que beaucoup d'élus ignorent que les crédits du concours particulier sont en quelque sorte les leurs. Qu'ils le sachent ou non, ils ne les perçoivent pas tout à fait comme tels, remarquant surtout que, pour les obtenir, des conditions doivent être remplies.

\footnotetext{
(75) Il s'agit bien des opérations subventionnées sur la $2^{\text {ème }}$ part du concours particulier, à l'exclusion de celles financées en 2000, 2001 et 2002 sur le titre VI (voir plus bas).
} 
A ce sujet, il y a d'ailleurs lieu de noter qu'une ambiguïté est entretenue - pour la bonne cause - par les DRAC qui laissent parfois les villes prendre pour des conditions de simples recommandations, telles que le recrutement de personnels qualifiés.

Comme on l'a précisé, l'enveloppe du concours particulier est non pas gérée de façon indivise à l'échelon national mais répartie entre les régions. Plus précisément, elle l'est "en fonction de la population de chaque région pondérée par le besoin d'équipement en matière de $B M "$; ce besoin d'équipement est "égal au quotient du rapport entre la population de la région et la surface totale, en mètres carrés, des BM de la région" ${ }^{(76)}$.

Plus proche de ses éventuels bénéficiaires, sans doute le dispositif gagne-t-il ainsi en visibilité et en attractivité.

Mais encore faut-il que des agents publics soient à même de l'accompagner d'une action pédagogique. C'est ce que faisait l'administration centrale du ministère de la Culture - en l'occurrence, la DLL - et qu'elle aurait pu continuer à faire. Mais, quels que soient la bonne volonté et le talent des personnes, elle l'aurait nécessairement fait moins efficacement que les CLL, plus nombreux et moins éloignés des éventuels bénéficiaires.

De ce point de vue, plus que la régionalisation des crédits proprement dite, ce qui a aidé au développement des BM, s'agissant de l'aide de l'État, sans doute est-ce plutôt la déconcentration, la mise en place des CLL.

Au chapitre de l'effet incitateur de la deuxième part du concours particulier, il doit être mentionné que les crédits attribués à ce titre sont versés en amont, avant le commencement de l’opération (éventuellement sur plusieurs exercices).

Cette facilité n'est pas sans présenter des risques. Des cas sont signalés de communes qui, ayant reçu des crédits pour construire ou aménager une BM, n'honorent pas leurs engagements : l'opération est différée, parfois jusqu'à laisser penser qu'elle n'aura jamais lieu, ou bien les locaux au titre desquels l'aide a été versée sont utilisés en totalité ou en partie à d'autres usages qu'une bibliothèque, etc.

Il n'a pas été dressé de récapitulatif de ces accidents. C'est dommage. Pour autant, ils ne semblent pas fréquents.

Plus que de notre sujet, à savoir l'efficacité du concours particulier, ces péripéties relèvent de celui du contrôle exercé par l'État sur l'utilisation de l'argent public et les suites qu'il entend lui donner.

\section{Pérennité et montant des aides}

Il y a lieu de remarquer, et que l'institution du concours particulier a sans doute protégé le soutien financier apporté par l'État à la construction et à l'équipement des BM, et que le caractère décisif de ce soutien a ses limites.

\footnotetext{
(76) Code général des collectivités territoriales, article R 1614-87.
} 
Le montant de la $2^{\text {ème }}$ part du concours particulier, et le taux de concours, ont évolué comme suit ${ }^{(77)}$.

\begin{tabular}{|c|c|c|c|}
\hline & $\begin{array}{c}\text { Montant } \\
\text { (loi de finances } \\
\text { initiale) }\end{array}$ & $\begin{array}{l}\text { Évolution par } \\
\text { rapport à l'année } \\
\text { précédente en \% }\end{array}$ & $\begin{array}{c}\text { Taux de } \\
\text { concours moyen } \\
\text { en } \%\end{array}$ \\
\hline 1986 & $92575662 \mathrm{~F}$ & & $35 \%$ \\
\hline 1987 & $124747825 \mathrm{~F}$ & $+34,75 \%$ & $39 \%$ \\
\hline 1988 & $146285123 \mathrm{~F}$ & $+17,26 \%$ & $38 \%$ \\
\hline 1989 & $165814508 \mathrm{~F}$ & $+13,35 \%$ & $40 \%$ \\
\hline 1990 & $169959871 \mathrm{~F}$ & $+2,50 \%$ & $39 \%$ \\
\hline 1991 & $182727955 \mathrm{~F}$ & $+7,51 \%$ & $36 \%$ \\
\hline 1992 & $190803888 \mathrm{~F}$ & $+4,42 \%$ & $38 \%$ \\
\hline 1993 & $184735827 \mathrm{~F}$ & $-3,18 \%$ & $37 \%$ \\
\hline 1994 & $188420544 \mathrm{~F}$ & $+2 \%$ & $39 \%$ \\
\hline 1995 & $191633863 \mathrm{~F}$ & $+1,70 \%$ & $38 \%$ \\
\hline 1996 & $198436865 \mathrm{~F}$ & $+3,55 \%$ & $39 \%$ \\
\hline 1997 & $201135706 \mathrm{~F}$ & $+1,36 \%$ & $41 \%$ \\
\hline 1998 & $203919162 \mathrm{~F}$ & $+1,38 \%$ & $39,13 \%$ \\
\hline 1999 & $209586566 \mathrm{~F}$ & $+2,78 \%$ & $36,37 \%$ \\
\hline 2000 & $211307271 \mathrm{~F}$ & $+0,82 \%$ & $35,23 \%$ \\
\hline 2001 & $218542897 \mathrm{~F}$ & $+3,42 \%$ & $36,65 \%$ \\
\hline 2002 & $\begin{array}{c}41258177 € \\
270635903 \mathrm{~F}\end{array}$ & $+23,84 \%$ & $?$ \\
\hline 2003 & $\begin{array}{c}42204652 € \\
276844369 \mathrm{~F}\end{array}$ & $+2,29 \%$ & $?$ \\
\hline
\end{tabular}

Les augmentations importantes que connaît l'enveloppe jusqu'en 1989 s'expliquent par la mise en place progressive du dispositif, autrement dit, par la progressivité du transfert des crédits concernés du ministère de la Culture au sein de la DGC.

L'enveloppe étant indexée sur la dotation globale de fonctionnement, on peut voir, sur le tableau, la deuxième part augmenter régulièrement, sauf en 1993, année qui la voit accuser une baisse avant de reprendre sa progression. Cette baisse est due à la constitution, cette année-là, de la troisième part. Inversement, c'est la suppression de celle-ci qui explique l'augmentation importante de l'enveloppe en 2003, les crédits rendus disponibles par cette suppression étant répartis selon la clef réglementaire : $65 \%$ pour la deuxième part et $35 \%$ pour la première.

Si la tendance du taux de concours paraît être à la baisse, celle-ci est lente et le taux a suffi, il suffit encore à convaincre des villes de réaliser les opérations subventionnées.

Pour autant, doivent être relevés les faits suivants :

$1^{\circ}$ ) Le pourcentage des dépenses pris en charge par le concours particulier n'est pas calculé sur la totalité des coûts, mais seulement sur une partie d'entre eux (en jargon, l'assiette subventionnable). Or, les coûts réels peuvent être notablement supérieurs.

$2^{\circ}$ ) Ainsi qu'il a été dit, le concours particulier fait l'objet d'une enveloppe prédéterminée, réévaluée chaque année de façon automatique en fonction de l'évolution du taux de la DGF.

Or, jusqu'ici, cette réévaluation n'a pas été tout à fait proportionnée aux besoins, tels qu'ils résultent du nombre des opérations et de l'enchérissement des coûts.

Comme pour la première part, il s'ensuit que la part de chacun tend à diminuer.

En fait, cette diminution se traduit de façon différente selon les régions. Dans certaines d'entre elles, le préfet, appliquant les textes à la lettre, estime que toutes les villes satisfaisant aux conditions minimales requises ont le droit d'émarger au concours particulier et de bénéficier du même taux. La conséquence est que la même baisse est appliquée à tous. Dans d'autres régions, le

\footnotetext{
${ }^{(77)}$ Source pour l'enveloppe et le pourcentage d'évolution : DGCL.
} 
préfet choisit au contraire de donner une prime à la qualité en privilégiant les opérations qui vont au-delà des conditions minimales requises. Mais c'est au détriment des autres.

Dans les deux cas, il y a des "perdants".

Si le concours particulier faisait l'objet d'une enveloppe nationale, le phénomène évoqué la baisse des taux suivant l'augmentation du nombre des opérations subventionnables - ne manquerait pas de se produire à ce niveau.

Mais peut-être est-il accentué par la répartition régionale de l'enveloppe. En effet, dans certaines régions où les opérations sont nombreuses, s'observe une tension sur les crédits, dépensés à $100 \%$. Et le concours particulier y est "hypothéqué" pour plusieurs années.

Les deux tableaux qui suivent illustrent les deux phénomènes évoqués : tension sur les crédits dans certaines régions; inégalité des taux de concours selon les régions.

Taux moyen d'utilisation des crédits délégués 1994-2001

NB : une case vide $=$ utilisation nulle

\begin{tabular}{|c|c|c|c|c|c|c|c|c|}
\hline & 1994 & 1995 & 1996 & 1997 & 1998 & 1999 & 2000 & 2001 \\
\hline Alsace & $100 \%$ & $100 \%$ & $54 \%$ & $85 \%$ & $100 \%$ & $99 \%$ & $99 \%$ & $21 \%$ \\
\hline Aquitaine & $100 \%$ & $100 \%$ & $100 \%$ & $100 \%$ & $100 \%$ & $100 \%$ & $100 \%$ & $100 \%$ \\
\hline Auvergne & $100 \%$ & $100 \%$ & $98 \%$ & $100 \%$ & $100 \%$ & $100 \%$ & $100 \%$ & $95 \%$ \\
\hline Bourgogne & $100 \%$ & $100 \%$ & $100 \%$ & $100 \%$ & $100 \%$ & $100 \%$ & $99 \%$ & $95 \%$ \\
\hline Bretagne & $67 \%$ & $68 \%$ & $190 \%$ & $99 \%$ & $100 \%$ & $102 \%$ & $86 \%$ & $100 \%$ \\
\hline Centre & $100 \%$ & $100 \%$ & $98 \%$ & $100 \%$ & $100 \%$ & $100 \%$ & $94 \%$ & $100 \%$ \\
\hline Champagne-Ardenne & $100 \%$ & $100 \%$ & $105 \%$ & $45 \%$ & $100 \%$ & $93 \%$ & $100 \%$ & $100 \%$ \\
\hline Corse & $3 \%$ & $2 \%$ & $32 \%$ & & $99 \%$ & $32 \%$ & & \\
\hline Franche-Comté & $98 \%$ & $95 \%$ & $100 \%$ & $100 \%$ & $100 \%$ & $100 \%$ & $62 \%$ & $39 \%$ \\
\hline Guadeloupe & $100 \%$ & $100 \%$ & $11 \%$ & $68 \%$ & & $100 \%$ & $100 \%$ & $100 \%$ \\
\hline Guyane & & & & & $100 \%$ & $100 \%$ & $100 \%$ & $100 \%$ \\
\hline Île-de-France & $100 \%$ & $100 \%$ & $40 \%$ & $80 \%$ & $100 \%$ & $100 \%$ & $100 \%$ & $100 \%$ \\
\hline Languedoc-Roussillon & $99 \%$ & $91 \%$ & $93 \%$ & $100 \%$ & $109 \%$ & $100 \%$ & $100 \%$ & $100 \%$ \\
\hline Limousin & $0 \%$ & $55 \%$ & $23 \%$ & $100 \%$ & $100 \%$ & $100 \%$ & $51 \%$ & $100 \%$ \\
\hline Lorraine & $100 \%$ & $98 \%$ & $90 \%$ & $100 \%$ & $82 \%$ & $64 \%$ & $100 \%$ & $87 \%$ \\
\hline Martinique & & $60 \%$ & & & & $11 \%$ & & \\
\hline Midi-Pyrénées & $99 \%$ & $100 \%$ & $93 \%$ & $99 \%$ & $100 \%$ & $105 \%$ & $83 \%$ & $100 \%$ \\
\hline Nord-Pas-de-Calais & $94 \%$ & $75 \%$ & $141 \%$ & $65 \%$ & $100 \%$ & $100 \%$ & $99 \%$ & $100 \%$ \\
\hline Basse-Normandie & $100 \%$ & $100 \%$ & $137 \%$ & $100 \%$ & $100 \%$ & $100 \%$ & $97 \%$ & $100 \%$ \\
\hline Haute-Normandie & $95 \%$ & $100 \%$ & $100 \%$ & $99 \%$ & $92 \%$ & $100 \%$ & $94 \%$ & $100 \%$ \\
\hline Pays-de-la-Loire & $100 \%$ & $100 \%$ & $49 \%$ & $100 \%$ & $100 \%$ & $100 \%$ & $100 \%$ & $100 \%$ \\
\hline Picardie & $92 \%$ & $95 \%$ & $99 \%$ & $100 \%$ & $98 \%$ & $100 \%$ & $99 \%$ & $100 \%$ \\
\hline Poitou-Charentes & $47 \%$ & $96 \%$ & $98 \%$ & $96 \%$ & $76 \%$ & $99 \%$ & $100 \%$ & $100 \%$ \\
\hline Provence-Alpes-Côte d'azur & $100 \%$ & $100 \%$ & $99 \%$ & $98 \%$ & $100 \%$ & $100 \%$ & $97 \%$ & $100 \%$ \\
\hline Réunion & $100 \%$ & $100 \%$ & & & $186 \%$ & $100 \%$ & $43 \%$ & $83 \%$ \\
\hline Rhône-Alpes & $100 \%$ & $100 \%$ & $100 \%$ & $100 \%$ & $98 \%$ & $103 \%$ & $100 \%$ & $100 \%$ \\
\hline Mayotte & & & & & & $100 \%$ & $100 \%$ & $100 \%$ \\
\hline
\end{tabular}

On remarquera que, d'après ce tableau, le taux d'utilisation de l'enveloppe a dépassé $100 \%$ dans certaines régions. Or, c'est impossible. Et - vérification faite - cet étrange résultat ne vient pas du fait que des crédits d'une autre nature auraient été ajoutés à l'enveloppe du concours particulier. Tant la source du tableau (DLL) que les DRAC concernées confirment que ce résultat est aberrant et interroge la fiabilité des données fournies et/ou du mode de calcul. 
Taux moyen de concours 1994-2001

NB : une case vide $=$ utilisation nulle.

\begin{tabular}{|c|c|c|c|c|c|c|c|c|}
\hline & 1994 & 1995 & 1996 & 1997 & 1998 & 1999 & 2000 & 2000 \\
\hline Alsace & $34 \%$ & $28 \%$ & $30 \%$ & $37 \%$ & $30 \%$ & $38 \%$ & $45 \%$ & $50 \%$ \\
\hline Aquitaine & $34 \%$ & $30 \%$ & $30 \%$ & $36 \%$ & $40 \%$ & $27 \%$ & $35 \%$ & $37 \%$ \\
\hline Auvergne & $50 \%$ & $50 \%$ & $50 \%$ & $50 \%$ & $50 \%$ & $50 \%$ & & $47 \%$ \\
\hline Bourgogne & $36 \%$ & $39 \%$ & $36 \%$ & $45 \%$ & $40 \%$ & $40 \%$ & $35 \%$ & $38 \%$ \\
\hline Bretagne & $36 \%$ & $35 \%$ & $35 \%$ & $35 \%$ & $35 \%$ & $25 \%$ & $23 \%$ & $27 \%$ \\
\hline Centre & $33 \%$ & $29 \%$ & $47 \%$ & $33 \%$ & $32 \%$ & $27 \%$ & $29 \%$ & $32 \%$ \\
\hline Champagne-Ardenne & $38 \%$ & $41 \%$ & $43 \%$ & $45 \%$ & $40 \%$ & $40 \%$ & $40 \%$ & $43 \%$ \\
\hline Corse & $48 \%$ & $48 \%$ & $50 \%$ & & $50 \%$ & $50 \%$ & & \\
\hline Franche-Comté & $38 \%$ & $34 \%$ & $43 \%$ & $37 \%$ & & $24 \%$ & $40 \%$ & $40 \%$ \\
\hline Guadeloupe & $45 \%$ & & $50 \%$ & $50 \%$ & & & $38 \%$ & $23 \%$ \\
\hline Guyane & & & & & & $25 \%$ & & $27,5 \%$ \\
\hline Île-de-France & $30 \%$ & $35 \%$ & $37 \%$ & $45 \%$ & $35 \%$ & $40 \%$ & $32 \%$ & $34 \%$ \\
\hline Languedoc-Roussillon & $39 \%$ & $36 \%$ & $35 \%$ & $36 \%$ & $35 \%$ & $32 \%$ & $28 \%$ & $30 \%$ \\
\hline Limousin & & $40 \%$ & $33 \%$ & $30 \%$ & $35 \%$ & $40 \%$ & $35 \%$ & $31 \%$ \\
\hline Lorraine & $37 \%$ & $36 \%$ & $27 \%$ & $32 \%$ & $40 \%$ & $40 \%$ & $38 \%$ & $35 \%$ \\
\hline Martinique & & $43 \%$ & & & & & & \\
\hline Midi-Pyrénées & $40 \%$ & $40 \%$ & $37 \%$ & $43 \%$ & $38 \%$ & $35 \%$ & $33 \%$ & $38 \%$ \\
\hline Nord-Pas-de-Calais & $40 \%$ & $39 \%$ & $38 \%$ & $41 \%$ & $45 \%$ & $45 \%$ & $44 \%$ & $42 \%$ \\
\hline Basse-Normandie & $40 \%$ & $39 \%$ & $39 \%$ & $50 \%$ & $41 \%$ & $25 \%$ & $33 \%$ & $31 \%$ \\
\hline Haute-Normandie & $39 \%$ & $39 \%$ & $37 \%$ & $40 \%$ & $35 \%$ & $40 \%$ & $35 \%$ & $32 \%$ \\
\hline Pays-de-la-Loire & $43 \%$ & $43 \%$ & $43 \%$ & $40 \%$ & $35 \%$ & $30 \%$ & $31 \%$ & $35 \%$ \\
\hline Picardie & $38 \%$ & $37 \%$ & $38 \%$ & $42 \%$ & $35 \%$ & $35 \%$ & $37 \%$ & $35 \%$ \\
\hline Poitou-Charentes & $40 \%$ & $40 \%$ & $38 \%$ & $40 \%$ & $40 \%$ & $40 \%$ & $30 \%$ & $34 \%$ \\
\hline Provence-Alpes-Côte d'azur & $47 \%$ & $46 \%$ & $45 \%$ & $47 \%$ & $50 \%$ & $50 \%$ & $43 \%$ & $41 \%$ \\
\hline Réunion & $42 \%$ & $33 \%$ & $35 \%$ & & $40 \%$ & $40 \%$ & $40 \%$ & NC \\
\hline Rhône-Alpes & $28 \%$ & $28 \%$ & $27 \%$ & $40 \%$ & $40 \%$ & $35 \%$ & $27 \%$ & $27 \%$ \\
\hline Mayotte & & & & & & & $75 \%$ & $70 \%$ \\
\hline
\end{tabular}

Dans le cadre de la loi de finances rectificative pour 1999, le ministère de la Culture a obtenu une dotation exceptionnelle de $96 \mathrm{MF}$ pour soutenir, à côté de la deuxième part du concours particulier, le même type d'opérations que celui-ci (investissements). Le même type d'opérations et aux mêmes conditions ${ }^{(78)}$, mais non les mêmes opérations.

En 2000 et 2001 ces crédits gérés par l'administration centrale (titre VI) - et réduits, chemin faisant, à un peu moins de $92 \mathrm{MF}$ - ont été répartis entre quelques régions identifiées comme en ayant plus particulièrement besoin.

\footnotetext{
${ }^{(78)}$ Circulaire $n^{\circ} 2000 / 025$ du 16 juin 2000 du directeur de cabinet aux préfets des régions concernées.
} 


\begin{tabular}{|c|c|c|}
\hline & 2000 & 2001 \\
\hline \multicolumn{3}{|l|}{ Alsace } \\
\hline Aquitaine & & $800000 \mathrm{~F} / 121959 €$ \\
\hline \multicolumn{3}{|l|}{ Auvergne } \\
\hline Bourgogne & $8,6 \mathrm{MF} / 1311061 €$ & $600000 \mathrm{~F} / 91469 €$ \\
\hline Bretagne & $11 \mathrm{MF} F / 1676939 €$ & $710000 \mathrm{~F} / 108239 €$ \\
\hline Centre & $6,56 \mathrm{MF} / 1000065 €$ & \\
\hline \multicolumn{3}{|l|}{ Champagne-Ardenne } \\
\hline \multicolumn{3}{|l|}{ Corse } \\
\hline Franche-Comté & $2,7 \mathrm{MF} / 411612 €$ & \\
\hline \multicolumn{3}{|l|}{ Guadeloupe } \\
\hline \multicolumn{3}{|l|}{ Guyane } \\
\hline Île-de-France & $13,5 \mathrm{MF} / 2058061 €$ & \\
\hline Languedoc-Roussillon & $4,2 \mathrm{MF} / 640285 €$ & \\
\hline \multicolumn{3}{|l|}{ Limousin } \\
\hline \multicolumn{3}{|l|}{ Lorraine } \\
\hline \multicolumn{3}{|l|}{ Martinique } \\
\hline Midi-Pyrénées & $10,4 \mathrm{MF} / 823224 €$ & \\
\hline Nord-Pas-de-Calais & $5,9 \mathrm{MF} / 899 \quad 449 €$ & \\
\hline Basse-Normandie & & $642000 \mathrm{~F} / 97872 €$ \\
\hline Haute-Normandie & $5,4 \mathrm{MF} / 823224 €$ & \\
\hline \multicolumn{3}{|l|}{ Pays-de-la-Loire } \\
\hline \multicolumn{3}{|l|}{ Picardie } \\
\hline \multicolumn{3}{|l|}{ Poitou-Charentes } \\
\hline Provence-Alpes-Côte d'azur & & $910000 \mathrm{~F} / 138729 €$ \\
\hline \multicolumn{3}{|l|}{ Réunion } \\
\hline Rhône-Alpes & $19 \mathrm{MF} / 2896531 €$ & $980000 \mathrm{~F} / 149400 €$ \\
\hline \multicolumn{3}{|l|}{ Mayotte } \\
\hline & $87,26 \mathrm{MF} / 13302701 €$ & $4642000 \mathrm{~F} / 707668 €$ \\
\hline
\end{tabular}

En outre, la région PACA a bénéficié, en 2002, toujours sur le titre VI, d'une dotation exceptionnelle du ministère de la Culture, indépendante de la précédente, de $1231578 €$ (8078 622 F).

Ces dotations ont permis d'augmenter le nombre d'opérations subventionnées dans les proportions suivantes.

NB : le fait que des opérations aient été soutenues en 2002 - outre celles qui ont eu lieu en PACA - alors que la dotation précitée de $91902000 \mathrm{~F}$ a été attribuée en 2000 et 2001 s'explique par des reports de crédits non dépensés.

\begin{tabular}{|l|c|c|c|c|c|c|c|c|c|}
\hline & \multicolumn{3}{|c|}{2000} & \multicolumn{3}{c|}{2001} & \multicolumn{2}{c|}{2002} \\
\hline & CP & T. VI & Total & CP & T. VI & Total & CP & T. VI & Total \\
\hline Bâtiments & 284 & 40 & 324 & 231 & 4 & 235 & 234 & 7 & 241 \\
\hline Equipement & 165 & 15 & 180 & 166 & 13 & 179 & 140 & 5 & 145 \\
\hline Informatique & 329 & 16 & 354 & 238 & 31 & 269 & 263 & 9 & 272 \\
\hline Patrimoine & 5 & 1 & 6 & 2 & 0 & 2 & 8 & 0 & 8 \\
\hline Bibliobus & 2 & 0 & 2 & 6 & 0 & 6 & 5 & 0 & 5 \\
\hline \multicolumn{1}{r}{ Total } & $\mathbf{7 8 5}$ & $\mathbf{7 2}$ & $\mathbf{8 5 7}$ & $\mathbf{6 4 3}$ & $\mathbf{4 8}$ & $\mathbf{6 9 1}$ & $\mathbf{6 5 0}$ & $\mathbf{2 1}$ & $\mathbf{6 7 1}$ \\
\hline
\end{tabular}

Initié en 2003, le programme "Les Ruches", dont il sera question plus loin, se situe lui aussi dans cette logique consistant à apporter un "renfort" au concours particulier.

Pour juger de l'efficacité de celui-ci, un autre élément doit être mentionné.

Bon nombre d'opérations ont bénéficié non seulement du concours particulier, mais d'autres aides provenant par exemple du département, de la région ou de l'Union européenne.

Dans le cas des villes que les BDP ont vocation à desservir, c'est-à-dire les villes de moins de 10000 habitants, la construction et l'équipement d'une BM sont susceptible de bénéficier deux fois du concours particulier: directement, par le biais du concours particulier communal ; indirectement, par le biais du remboursement au département, au titre du concours particulier 
départemental, d'une partie de l'aide qu'il aura attribuée à la ville en question pour la même opération.

C'est un domaine peu étudié que celui du cumul des aides et de leur effet sur la décision de construire. Seul, le concours particulier suffirait-il à emporter cette décision ?

$3^{\circ}$ ) Le fait que l'enveloppe du concours particulier soit répartie entre les régions - ou plus précisément, les critères retenus pour cette répartition - font que, tandis que certaines régions disposent de crédits insuffisants, d'autres en ont de reste ${ }^{(79)}$.

Il importe d'ajouter

- que ces crédits non utilisés ne sont pas tout à fait perdus, ils viennent abonder l'enveloppe globale de l'année suivante.

- qu'il est loisible à une DRAC de demander le report des sommes non dépensées dans son enveloppe propre (depuis l'institution du concours particulier, les demandes faites en ce sens n'ont pas dépassé 4 selon la DLL).

En l'état, le dispositif du concours particulier n'est donc pas sans présenter des faiblesses.

D'un autre côté, l'expérience montre que les budgets des ministères ne sont pas assurés d'une augmentation régulière ni même d'une reconduction. Et de ce point de vue, l'application en cours de la loi organique relative aux lois de finance (lolf) a toute chance d'accentuer, pour le meilleur et pour le pire, ce phénomène.

Par comparaison, l'insertion du budget d'aide à la construction et à l'équipement des BM dans la DGC, qui est régulièrement réévalué, apparaît comme garantissant mieux leur maintien et leur progression, et alors même que celle-ci ne suit manifestement pas la progression des opérations et/ou des coûts.

Pour autant, le "combat de la lecture publique" n'est pas encore gagné. Dans son rapport pour 1998-1999, le Conseil supérieur des bibliothèques écrit : "On peut affirmer, en observant la trajectoire ascendante suivie depuis les années 1960, que notre pays se trouve à mi-chemin dans son entreprise de construction d'un réseau de lecture publique cohérent et efficace".

Quantité de beaux bâtiments, parfois prestigieux, ne doivent pas faire illusion. Après tout, qu'on dénombre entre 3000 et 4000 BM, cela signifie certes qu'il y a trois fois plus de BM qu'il y a vingt ans ; mais cela signifie aussi qu'au moins 32000 communes n'ont toujours pas de BM. D'autre part, même dans des villes qui bénéficient d'une centrale récente, la desserte de proximité n'est pas à la hauteur ; les annexes sont insuffisantes, en quantité et en qualité.

Des disparités, parfois très marquées, s'observent entre les régions.

Des trous que présente encore le territoire du point de vue des bâtiments, le ministère de la Culture est si conscient que, à partir de 2003, il a décidé d'encourager la création de bibliothèques dans deux types de zones : les "zones rurales éloignées des villes-centre" et les "quartiers urbains périphériques" ${ }^{800}$. Il s'agit du programme dit "les ruches".

Pour le financer, le concours particulier est utilisé. Il y a été recouru à hauteur de 13 millions d'euros en 2003.

Mais un autre budget, celui-là géré par le ministère, a été dégagé en complément. Il s'agit de crédits du titre VI, consacré à l'investissement, celui auquel il avait déjà été recouru en 2000 et 2001 pour venir s'ajouter à la deuxième part du concours particulier dans certaines régions, ainsi

\footnotetext{
(79) Il y aurait lieu de s'interroger sur les raisons pour lesquelles certaines régions ne dépensent pas l'intégralité de leurs crédits, particulièrement quand ce n'est pas exceptionnel.

${ }^{(80)}$ Le ministre de la Culture et de la communication aux préfets de région (DRAC), note technique $\mathrm{n}^{\circ} 2003 / 015$ du $1^{\text {er }}$ octobre 2003 (Bulletin officiel du ministère, $n^{\circ}$ 139, janv. 2004, p. 21-24). Pour des compléments et un premier bilan, voir $F$. Rouyer-Gayette, "Les Ruches", BBF, t. 49, 2004, n² 2, p. 24-29.
} 
que de crédits du titre IV, consacré au fonctionnement. En effet, outre une aide à la construction et à l'équipement, est attribuée une aide à la constitution de fonds multimédia et au recrutement de personnel qualifié. ${ }^{\left({ }^{81}\right)}$

En 2003, les crédits en question ont représenté 950000 euros pour le titre $\mathrm{VI}$ et 800000 euros pour le titre IV. En 2004, ils se monteront à 2,25 millions d'euros. Par décision du comité interministériel à l'aménagement du territoire (septembre 2003), s'y ajouteront, au bénéfice des zones rurales, 1,5 millions d'euros du fonds national d'aménagement du territoire.

En 2003, a été financée une centaine de projets, $80 \%$ en zone rurale et $20 \%$ en zone urbaine ${ }^{(82)}$. 85 concernent des bâtiments. 45 emplois ont fait l'objet d'une aide.

Du point de vue de l'histoire des politiques publiques en matière de lecture publique, sous le rapport de la décentralisation, cette initiative présente un vif intérêt. En effet, elle consiste pour l'État à prendre acte des limites de la seule initiative des collectivités territoriales - même stimulée par le concours particulier et une action pédagogique. Et à reprendre la main, crédits additionnels à l’appui, pour y remédier.

S'agissant des 12 BMVR qui ont ouvert ou vont ouvrir grâce à la troisième part du concours particulier, elles sont, dans l'ensemble, réussies.

Elles présentent toutefois un point faible : la "vocation régionale". En effet, les BMVR ne rendent pas tous les services attendus en matière de coopération. Outre les difficultés que rencontre d'ordinaire la coopération, il n'est pas très difficile d'en comprendre la raison. L'État a limité son aide à la construction et à l'équipement. Et il a tenu aux villes le raisonnement suivant : en échange de cette aide, vous allez assumer des missions coopératives. On peut traduire la réponse (ou la non-réponse) des villes de la manière suivante : Nous vous remercions beaucoup de nous avoir aidés à construire et à équiper notre bibliothèque. Mais, d'abord, nous avons acquitté une part non négligeable des factures, ensuite, pour remplir les missions coopératives que vous nous demandez d'assumer, il faut d'autres moyens, des emplois, des budgets de fonctionnement. Pourquoi voulez-vous que nous utilisions les nôtres, ceux qui sont financés pour partie par nos contribuables, au profit des citoyens d'autres villes?

\section{b) Le fonctionnement}

Globalement, le fonctionnement des BM n'est pas à la hauteur des équipements.

Un des principaux points faibles, est constitué par les plages d'ouverture hebdomadaires. Depuis de longues années, elle est d'un peu plus de 19 heures sur un peu plus de 4 jours. C'est dérisoire. Il est permis de penser que cette étroitesse des plages d'ouverture n'est pas seulement une question de moyens - en l'occurrence, de moyens en personnel - mais aussi d'organisation et de "culture" du service public. Le nombre global des agents n'en est pas moins en deçà des besoins ; et de ce point de vue, l'application des 35 heures (parfois, le temps de travail hebdomadaire est inférieur à 35 heures) n'a rien arrangé quand elle n'a pas été compensée par des recrutements. D'un côté, les BM ont besoin de personnel pour fonctionner. D'un autre côté, les villes ne croient pas devoir ou pouvoir recruter davantage de fonctionnaires. Elles résolvent le dilemme en engageant des agents plus ou moins temporaires, plus ou moins qualifiés.

Dans ces conditions, il est permis de penser

1) que le fait que l'État rembourse aux villes une partie de leurs dépenses va dans le sens d'un meilleur fonctionnement des BM ;

\footnotetext{
(81) S'agissant de l'investissement, une bibliothèque peut bénéficier à la fois de la DGD et du titre VI, mais pas pour les mêmes types d'opérations (construction, mobilier, équipement informatique).

${ }^{(82)}$ Les projets doivent être "portés prioritairement par des EPCI" et les subventions seront allouées "dans un premier temps" au bénéfice de "dépenses d'investissement concourant à doter [les médiathèques en question] d'outils informatiques et de ressources multimédia" (note 04-095 du directeur du Livre et de la lecture et du délégué à l'Aménagement du territoire et à l'Action régionale aux préfets de région, 17 mars 2004.
} 
2) qu'il en est de même du fait que ce remboursement soit identifié comme tel et donc que le concours particulier - dès lors que les crédits du ministère de la culture devaient être versés dans la DGC - est un dispositif plus pertinent que ne l'aurait été son absence.

Ce dispositif n'en appelle pas moins deux observations :

- Le taux moyen de remboursement est bas - 3, $29 \%$ en $2003{ }^{\left({ }^{83}\right)}$ - et baisse tendanciellement depuis $1990^{\left({ }^{84}\right)}$. La raison en est simple. Le nombre de BM ne cesse d'augmenter, ainsi que le niveau des dépenses. Or, si l'enveloppe à répartir est régulièrement réévaluée (ainsi qu'il a été dit, elle est indexée sur la DGF), elle ne l'est pas en proportion. Pour employer l'image usuelle: l'accroissement de la taille du gâteau ne suit pas celle du nombre des convives; aussi la part de chacun est-elle de plus en plus réduite. C'est ce que montre le tableau suivant ${ }^{855}$.

Comme dans le cas de la deuxième part, l'enveloppe baisse en 1993 et pour la même raison: la constitution de la $3^{\text {ème }}$ part. Et elle augmente en 2002 pour la même raison: la suppression de la $3^{\text {ème }}$ part.

\begin{tabular}{|c|c|c|c|c|}
\hline & $\begin{array}{c}\text { Montant } \\
\text { à répartir } \\
\text { (Lois de finances) }\end{array}$ & $\begin{array}{l}\text { Évolution par } \\
\text { rapport à l'année } \\
\text { précédente }\end{array}$ & $\begin{array}{l}\text { Villes } \\
\text { éligibles }\end{array}$ & $\begin{array}{c}\text { Taux de } \\
\text { remboursement }\end{array}$ \\
\hline 1986 & $49848433 \mathrm{~F}$ & & 589 & $3,45 \%$ \\
\hline 1987 & $67171906 \mathrm{~F}$ & $+34,75 \%$ & 639 & $4,17 \%$ \\
\hline 1988 & $78768912 \mathrm{~F}$ & $+17,26 \%$ & 736 & $5,50 \%$ \\
\hline 1989 & $89284735 \mathrm{~F}$ & $+13,35 \%$ & 773 & $6,25 \%$ \\
\hline 1990 & $91516853 \mathrm{~F}$ & $+2,50 \%$ & 814 & $5,90 \%$ \\
\hline 1991 & $98391931 \mathrm{~F}$ & $+7,51 \%$ & 871 & $5,30 \%$ \\
\hline 1992 & $102740555 \mathrm{~F}$ & $+4,42 \%$ & 870 & $4,84 \%$ \\
\hline 1993 & $99473138 \mathrm{~F}$ & $-3,18 \%$ & 977 & $4,19 \%$ \\
\hline 1994 & $101462000 \mathrm{~F}$ & $+2 \%$ & 1059 & $4,08 \%$ \\
\hline 1995 & $103187465 \mathrm{~F}$ & $+1,70 \%$ & 1097 & $3,68 \%$ \\
\hline 1996 & $106850620 \mathrm{~F}$ & $+3,55 \%$ & 1128 & $3,53 \%$ \\
\hline 1997 & $108303842 \mathrm{~F}$ & $+1,36 \%$ & 1166 & $3,31 \%$ \\
\hline 1998 & $109802626 \mathrm{~F}$ & $+1,38 \%$ & 1235 & $3,35 \%$ \\
\hline 1999 & $112854305 \mathrm{~F}$ & $+2,78 \%$ & 1264 & $3,30 \%$ \\
\hline 2000 & $113780838 \mathrm{~F}$ & $+0,82 \%$ & 1361 & $3,05 \%$ \\
\hline 2001 & $117676944 \mathrm{~F}$ & $+3,4 \%$ & 1401 & $3,00 \%$ \\
\hline 2002 & $\begin{array}{l}22215942 € \\
145727024 \mathrm{~F}\end{array}$ & $+23,84 \%$ & 1556 & $3,36 \%$ \\
\hline 2003 & $\begin{array}{c}22725582 € \\
149070045 \mathrm{~F}\end{array}$ & $+2,29 \%$ & 1645 & $3,29 \%$ \\
\hline
\end{tabular}

La logique du système aurait voulu qu'un taux minimal fût garanti quel que soit le nombre des BM bénéficiaires et le montant de leurs dépenses, l'objectif étant de favoriser les constructions et les dépenses prises en compte, en tant qu'elles tendent à élever la qualité du service.

Bien entendu, pour prendre l'exacte mesure de l'aide financière apportée par l'État au fonctionnement des $B M$, il y aurait lieu de tenir compte non seulement de la première part du concours particulier mais de l'ensemble des subventions qu'il leur attribue à ce titre. Si surprenant que cela soit, ce calcul n'a pas été effectué.

Démontrerait-il que, à défaut de pouvoir augmenter significativement le montant de la première part du concours particulier, le ministère de la Culture a du moins fait progresser notablement les crédits d'aide aux BM dont il continue à disposer directement ?

\footnotetext{
${ }^{(83)}$ Décret $n^{\circ}$ 2003-1027 du 23 octobre 2003 (Journal officiel du 30).

(84) S'agissant de la période antérieure à 1981-1982, A.-M. Bertrand donne le chiffre de 6,85\% pour la part prise par l'État au fonctionnement des BM en 1977, 5,42\% en 1980 ("Les bibliothèques municipales dans les années 80 : un développement spectaculaire, mais inachevé", $B B F$, t. $37,1992, n^{\circ} 4$, p. 8) ; et cette part monte à $19,26 \%$ en 1982 avant de redescendre de façon continue (dès 1985, elle n'est déjà plus que de $7,70 \%$ ). Mais quels crédits sont-ils pris en compte par ces pourcentages? Prennent-ils en compte le traitement des personnels d'État, qui, depuis 1986, s'ajoute au concours particulier?

${ }^{(85)}$ Source pour l'enveloppe et le pourcentage d'évolution : DGCL.
} 
De ce point de vue, on a dû borner les investigations aux crédits attribués par le CNL pour l'achat de livres. Le résultat en est le suivant.

Il est à noter que, dans les tableaux fournis, les bibliothèques de districts et de syndicats intercommunaux sont comptabilisées jusqu'en 1998 dans la rubrique des bibliothèques "diverses" et ne sont donc pas comprises dans les chiffres ci-dessous. En 1999, elles ne sont pas mentionnées dans les tableaux. En 2000, 2001 et 2002, elles sont bien comprises dans les chiffres ci-dessous.

\begin{tabular}{|c|c|c|c|}
\hline & $\begin{array}{c}\text { BM } \\
\text { Bénéficiaires }\end{array}$ & $\begin{array}{c}\text { Montant } \\
\text { total }\end{array}$ & $\begin{array}{c}\text { Montant moyen } \\
\text { par BM }\end{array}$ \\
\hline 1980 & 578 & $?$ & $?$ \\
\hline 1981 & 581 & $?$ & $?$ \\
\hline 1982 & 673 & $?$ & $?$ \\
\hline 1983 & 754 & $25856723 \mathrm{~F}$ & $34292 \mathrm{~F}$ \\
\hline 1984 & 915 & $29909192 \mathrm{~F}$ & $32687 \mathrm{~F}$ \\
\hline 1985 & 1023 & $31169989 \mathrm{~F}$ & $30469 \mathrm{~F}$ \\
\hline 1986 & 814 & $21404700 \mathrm{~F}$ & $26295 \mathrm{~F}$ \\
\hline 1987 & 805 & $20774519 \mathrm{~F}$ & $25806 \mathrm{~F}$ \\
\hline 1988 & 1052 & $22321021 \mathrm{~F}$ & $21217 \mathrm{~F}$ \\
\hline 1989 & 1007 & $26025546 \mathrm{~F}$ & $25844 \mathrm{~F}$ \\
\hline 1990 & 973 & $32918930 \mathrm{~F}$ & $33832 \mathrm{~F}$ \\
\hline 1991 & 785 & $23968500 \mathrm{~F}$ & $30533 \mathrm{~F}$ \\
\hline 1992 & 581 & $22531100 \mathrm{~F}$ & $38779 \mathrm{~F}$ \\
\hline 1993 & 559 & $19276600 \mathrm{~F}$ & $34484 \mathrm{~F}$ \\
\hline 1994 & 446 & $19139000 \mathrm{~F}$ & $42912 \mathrm{~F}$ \\
\hline 1995 & 414 & $18892000 \mathrm{~F}$ & $45632 \mathrm{~F}$ \\
\hline 1996 & 359 & $14444200 \mathrm{~F}$ & $40234 \mathrm{~F}$ \\
\hline 1997 & 373 & $13495150 \mathrm{~F}$ & $36180 \mathrm{~F}$ \\
\hline 1998 & 403 & $14800500 \mathrm{~F}$ & $36725 \mathrm{~F}$ \\
\hline 1999 & $447(86)$ & $18064000 \mathrm{~F}$ & $40411 \mathrm{~F}$ \\
\hline 2000 & 500 & $20622350 \mathrm{~F}$ & $41244 \mathrm{~F}$ \\
\hline 2001 & 590 & $22652770 \mathrm{~F}$ & $38394 \mathrm{~F}$ \\
\hline 2002 & 495 & $3353920 €$ & $6775 €$ \\
\hline 1996 & $22000273 \mathrm{~F}$ & $44445 \mathrm{~F}$ \\
\hline
\end{tabular}

Pour les années 1980 à 1982, le montant des crédits attribués n'est pas disponible par type de bibliothèque. Nous savons toutefois que ce montant était en 1980 de $24965100 \mathrm{~F}$ tous types de bibliothèques confondus et que cette somme représentait une augmentation de $15,48 \%$ par rapport à 1979. En 1981, il était de $31784368 \mathrm{~F}(+26,87 \%)$ et en 1982 de $39342800 \mathrm{~F}(+23,78 \%)$.

Le commentaire qu'appelle le tableau s'impose : par rapport aux années 1980, le budget global ayant diminué, c'est la réduction du nombre des bénéficiaires qui a permis de limiter l'érosion de l'aide apportée à chacun d'eux.

Revenons-en au concours particulier.

Aujourd'hui, donc, s'il est vrai que les BM, nouvellement bâties ou non, accèdent au concours particulier en atteignant un certain niveau de dépenses, d'une part, ce niveau est bas, d'autre part, les autres BM voient le niveau de l'aide qui leur est apportée baisser. Ce qui, à rebours de l'objectif visé par le dispositif, pourrait dissuader les villes de construire, ou les porter à réduire le budget de fonctionnement de leur BM.

Au ministère de la Culture et dans les milieux professionnels, s'expriment, en présence de ce phénomène, deux points de vue. Le premier - majoritaire et même sans doute largement majoritaire - consiste à considérer que, même faible, l'apport du concours particulier est un apport, perçu comme tel par les villes ; sur le plan symbolique, il présente aussi un avantage en manifestant que, pour l'État, les bibliothèques ne sont pas n'importe quel service. Le second point de vue - minoritaire - consiste à s'interroger d'autant plus sur l'efficacité d'une aide aussi modeste qu'il s'agit d'un remboursement acquitté avec un délai de deux ans.

(86) Pour 1999, le chiffre donné semble désigner non les bénéficiaires, mais les dossiers acceptés ; or, il peut y en avoir plusieurs par BM. 
- Seules bénéficient du remboursement les villes qui atteignent un certain niveau de dépenses. Elles sont au nombre de 1645 en 2003. Or, il reste à démontrer (peut-être est-ce démontrable, mais cela reste à démontrer) que la perspective d'un remboursement - au taux de $3 \%$ mais aussi de 10 ou de $15 \%$ - constitue une véritable incitation pour les villes à accroitre la dotation de leur BM, qu'il s'agisse de celles qui n'en bénéficient pas ou de celles qui ont déjà atteint le seuil de remboursement.

Remboursement ou pas, $3 \%$ ou $10 \%$, une ville qui croit à l'utilité culturelle et sociale de la BM ne lui donnera-t-elle pas de toute façon les moyens de fonctionner au mieux? Une ville qui n'y croit pas se laissera-t-elle convaincre par une aide de $3 \%$ ou de $10 \%$ et même de $15 \%$ ?

\section{c) Les conservateurs}

Quels ont été les effets de la possibilité ouverte aux villes possédant une BMC de créer, à côté des conservateurs mis à disposition par l'État, des postes de conservateurs territoriaux?

Au 31/12/02, on dénombrait dans les 54 BMC 85 conservateurs territoriaux pour 184 conservateurs d'État ${ }^{(87)}$. Il est intéressant de relever que, à l'origine, l'État s'est contenté de laisser des villes créer des postes de conservateurs territoriaux sans le vouloir vraiment ; il s'est trouvé un peu débordé. Ce n'est qu'ensuite que, réticent à fournir les BMC en emplois d'État, il a encouragé ces créations. Et d'ailleurs, en oubliant d'abroger le texte qui réservait en principe les BMC aux conservateurs d'État.

Malgré tout, le nombre des postes de conservateurs territoriaux que les villes possédant une BMC sont autorisées à créer - et dont on considère donc qu'il est approprié au nombre d'habitants est trois fois plus important (231) que le nombre de postes actuel ${ }^{(88)}$. Certaines villes n'ont créé aucun poste de conservateur territorial.

85 postes créés dans 54 villes en une vingtaine d'années, il est loisible à chacun d'estimer que c'est peu ou que c'est beaucoup. Là encore, s'agissant d'évaluer les effets de la décentralisation, il faut se demander si l'État aurait fait mieux. C'est peu probable. C'est d'autant moins probable que, depuis la décentralisation, il n'a plus créé de postes de conservateurs au profit des BMC ${ }^{(89)}$.

De cette absence de création de postes d'État, des villes font d'ailleurs reproche à celui-ci. On a en même vu une, ces dernières années, demander le classement de sa BM pour bénéficier d'une telle aide ${ }^{\left({ }^{90}\right)}$. Et ce alors qu'aucune BM n'a plus été classée depuis 1972 et que ce dispositif est aux antipodes de la philosophie de la décentralisation.

Il est à noter que non seulement l'État ne crée plus de postes d’État, mais que, pour diverses raisons, il peine à pourvoir ceux qui existent. Certaines des villes qui pâtissent de cet état de fait demandent que, dans ces conditions, l'État se livre à leur profit à une opération de même type que celle qui a été conduite lors de la décentralisation des BCP. A savoir, la transformation des postes d'État en postes territoriaux, l'État transférant aux communes le montant des traitements correspondants; de telle sorte que les villes concernées puissent recruter des conservateurs territoriaux.

Quant à la situation, aujourd'hui, des conservateurs d'État dans les BMC, et plus précisément sous le rapport des fonctions de direction, deux observations peuvent être formulées.

\footnotetext{
(87) Informations communiquées par la DLL. Selon les réponses faites à l'enquête statistique annuelle du ministère de la Culture, il peut ne pas s'agir du nombre des emplois, mais du nombre des personnes en poste dans l'année. L'ordre de grandeur n'en est pas moins exact.

${ }^{(88)}$ En termes de besoins calculés d'après la population, il s'agit d'un chiffre minimal, car quelques communes possédant une $B M C$, qui n'ont pas souhaité envisager de créations, ne sont pas prises en compte dans ce total.

(89) Autre chose est la redistribution de certains postes au sein du groupe des BMC - autrement dit, le fait de prélever un poste sur le contingent d'une ville pour en faire bénéficier une autre. Je connais au moins un cas de ce type.

(90) Il s'agit de Strasbourg. La demande n'a pas abouti.
} 
1) Dans la quasi-totalité des villes où ont été créés des postes de conservateurs territoriaux, le directeur reste un conservateur d’État ${ }^{(91)}$.

Non exclusives les unes des autres, plusieurs raisons sont susceptibles de l'expliquer : crédit fait à l'État et à son personnel du point de vue du "professionnalisme" ; stratégie (un conservateur d'État n'attirera-t-il pas des concours plus substantiels de la part de l'État qu'un conservateur territorial ?) ; appartenance des directeurs ou des candidats à la direction au corps des conservateurs généraux, hiérarchiquement situé au-dessus de celui des conservateurs et sans équivalent dans la fonction publique territoriale, ou, s'ils ne sont pas déjà conservateurs généraux, vocation de ces conservateurs à accéder à ce corps supérieur. Sans oublier le poids de l'habitude. Et la méconnaissance du fait que la direction des BMC n'est pas réservée aux conservateurs d'État.

Quoi qu'il en soit, il est à noter que si un cadre d'emploi de conservateur général venait à être créé dans la fonction publique territoriale, ainsi qu'il est par ailleurs très souhaitable, la concurrence faite aux conservateurs d'État par les conservateurs territoriaux pour la direction des BMC se ferait plus sensible. évolué.

2) La situation des conservateurs d'État-directeurs au sein de l'administration municipale a

Cette situation a toujours été ambiguë. Fonctionnaire d'État mis à la disposition d'une ville, un conservateur d'État avait pour ainsi dire deux maîtres : l'État et le maire.

Dans certains cas, le fait que le directeur soit un conservateur d'État n'a pas empêché le développement de la BM et a même pu y aider. Dans d'autres cas, il a pu y faire obstacle. Ou a pu servir à justifier, à tort ou à raison, un immobilisme.

Tantôt féconde et tantôt bloquante, cette ambiguïté demeure.

Ce qui a changé c'est que

- du directeur de la BM, conservateur d'État ou non, le maire attend désormais le même degré d'intégration dans sa politique que des autres chefs de service municipaux. Parce que l'autonomie des communes s'est accrue, et avec elle la latitude des maires. Mais c'est aussi, dans le domaine qui nous occupe, la rançon de l'essor des politiques culturelles et de la place qu'y ont prises, le cas échéant, les bibliothèques.

Dans ce contexte, plusieurs affaires le montrent (des affaires qui tendent à se multiplier ?), un maire a toute chance aujourd'hui de se montrer moins patient qu'autrefois si un directeurconservateur d'État ne lui paraît pas correspondre, à tort ou à raison, à son attente.

- L'État, qui rémunère les directeurs en question, n'en est pas moins lui aussi, plus qu'autrefois, au diapason de cette conception "intégrative". Tant que les conservateurs d'État sont mis à la disposition des villes, il considère que leur supérieur hiérarchique est bien le maire. C'est d'ailleurs au maire que, quand un poste de conservateur d'État est vacant dans une BMC, l'État laisse le soin de choisir parmi les candidats. (Il en use désormais de même avec les présidents d'université quand il s'agit de choisir un directeur pour le SCD).

Soit spontanément, soit parce que leurs relations avec le maire sont devenues difficiles, des conservateurs d'État-directeurs sont encore parfois tentés d'opposer leur légitimité de fonctionnaires de l'État aux consignes de la municipalité. Cette tactique ne laisse rien augurer de bon quant à leur avenir dans la commune.

Depuis au moins le rapport Béghain (1989), il est question de clarifier la situation des conservateurs d'État dans les BMC en précisant leurs missions dans un texte contractuel que l'État

\footnotetext{
(91) Je ne connais, au moment où ces lignes sont écrites, que deux cas de BMC dirigée par un conservateur territorial (Limoges, Roubaix). Une troisième pourrait les rejoindre (Dole), la ville, constatant que le poste de conservateur d'Étatdirecteur restait vacant, ayant créé un poste de conservateur territorial pour recruter un directeur.
} 
signerait avec les villes ${ }^{(92)}$. Ce dispositif n'a pas été mis en place. Pas seulement, mais sans doute aussi parce que l'État s'interroge sur les dispositions qu'il y a lieu de faire figurer dans ce texte. Et sur son rôle.

\subsubsection{Les $B D P$}

a) Les BDP, services départementaux

\section{$>$ La départementalisation : une opération profitable aux BDP?}

La départementalisation des BCP leur a-t-elle été profitable ? Cette question en recouvre au moins deux.

- La décision de départementaliser les BCP a-t-elle aidé à ce que le réseau soit complété, ses équipements améliorés?

La réponse à cette question ne saurait être que positive.

En 1981, 35 ans après la création des BCP - et sans compter Paris et les 3 départements de la petite couronne, non plus que, outre-mer, la Guyane et Saint-Pierre-et-Miquelon - 17 départements en attendent encore une. Et les moyens dont disposent les BCP existantes sont très inégaux. Ainsi 36 sont-elles "toujours installés dans des locaux provisoires" inadaptés; et 14 ne disposent-elles que d'un seul bibliobus ${ }^{\left({ }^{93}\right)}$.

Tôt ou tard, l'État aurait fini par doter chaque département d'une BCP. Et peut-être d'un parc amélioré de bâtiments et de véhicules. Mais dans quel délai?

Si la poursuite de la couverture du territoire en BCP connaît une accélération décisive, si les équipements sont mis à niveau, c'est bien à la faveur de la départementalisation.

Mais les choses auraient pu se passer différemment, le réseau aurait pu être transféré en l'état, c'est-à-dire incomplet et insuffisant. Il resterait donc à expliquer pourquoi l'opération a finalement connu un développement positif.

Sans doute parce que compte tenu des circonstances, tout le monde y a trouvé son compte. Tout le monde :

- le ministère de la Culture qui, dès lors qu'il y avait transfert et quoi qu'il puisse en penser, s'est attaché à garantir - autant que faire se pouvait - le dispositif et à lui donner toutes ses chances en le complétant et en le mettant à niveau. Au nombre des raisons qui expliquent que ses représentations sur ce point aient été entendues au sein du gouvernement, il faut sans doute compter le crédit du ministre et de son département auprès de l'Élysée.

- les départements, probablement d'autant mieux disposés (d'autant moins réticents ?) à accepter cette responsabilité nouvelle que les services transférés seraient en état de marche.

Du côté de Bercy, n'a-t-on pas d'autant mieux accepté de dégager les budgets nécessaires qu'il s'agissait d'investissements - de dépenses temporaires qui, compte tenu du transfert, ne devaient pas générer pour l'État de dépenses de fonctionnement pérennes ?

- Quel sort les départements ont-ils réservé aux BDP, la départementalisation a-t-elle profité aux moyens de celles-ci ?

Lorsque, dans l'entre-deux guerres, les milieux professionnels s'attachent à concevoir le mode de desserte du milieu rural le plus pertinent, ce n'est pas vers le département que se tourne

\footnotetext{
(92) Rappelons que les emplois des conservateurs d'État sont implantés dans les Drac correspondantes. Sur la situation des conservateurs d'État des BMC et la nécessité de clarifier leurs relations avec les villes, voir le rapport de l'Inspection pour 1996, p. 49-54.

${ }^{(93)}$ Voir le rapport Vandevoorde, 1982, annexes, première partie, II.
} 
leur regard: "[...] La profession défendait plutôt l'idée de micro-régions indépendantes de tout cadre administratif [...]" (94).

Rétrospectivement, il est permis de penser que l'adoption du cadre départemental a préservé le dispositif représenté par les BCP. En effet, la décentralisation survenant, que serait-il advenu de services que des collectivités locales ne pouvaient pas s'approprier ? De l'intérêt que les $B C P$ puissent s'adosser à une collectivité précise, les créateurs des BCP étaient d'ailleurs conscients qui, en 1946, justifiaient le choix du cadre départemental, entre autres raisons, par la suivante: "Constituer des divisions géographiques sans base administrative [...] eût amené des difficultés de tous ordres et eût vraisemblablement réduit l'aide des services départementaux avec lesquels les $B C P$, telles qu'elles ont été constituées, sont continuellement en liaison" ${ }^{995}$.

Avec la Cour des comptes et le ministre de la Culture ${ }^{\left({ }^{96}\right)}$, il faut souligner que, quand la décentralisation est décidée, seuls les services d'archives et les BCP sont désignés comme relevant de collectivités territoriales bien précises. A ces exceptions près, la culture, sous tous ses aspects, est implicitement considérée comme relevant indistinctement de tous les types de collectivités. Il devait d'ailleurs en résulter, il en résulte un enchevêtrement d'interventions qui n'est pas sans présenter des redondances ni des lacunes. Et pour le ministère de la Culture, une difficulté à identifier les partenaires appropriés qui l'a conduit à multiplier les dispositifs contractuels.

De cette désignation claire d'une collectivité "en charge", la lecture publique en milieu rural était mise, par-là même, en situation de profiter.

Pour autant, il n'allait pas de soi que les conseils généraux acceptent les BCP de bon gré et se les incorporent.

Pour qu'ils le fassent, les BCP possédaient plusieurs atouts. Leur champ de compétence se trouvait coïncider avec les limites du département ; chacune d'elle avait vocation à desservir tout le département, et rien que le département. En outre, elles avaient pour vocation d'intervenir auprès des petites communes, préoccupation majeure des départements, les villes d'une certaine importance ayant moins besoin de leur aide.

Au demeurant, dès avant leur départementalisation, les BCP avaient noué des liens avec les conseils généraux, en particulier à travers des associations d'amis.

Sur un autre plan, l'État remettait aux départements les clefs de bâtiments neufs ou agrandis et rénovés.

Ces atouts n'étaient que des atouts. Quel sort les départements ont-ils réservé aux BDP ?

Dans la littérature professionnelle et politico-administrative, le transfert des BCP aux départements est généralement présenté comme positif voire très positif. On ne saurait être ni favorable ni hostile à cette appréciation. Il faut seulement constater que les constats sont fonction des critères retenus, qui sont eux-mêmes parfois fonction de positions a priori.

Pour déterminer si la départementalisation des BDP est une réussite, la première tâche à accomplir serait sans doute de s'entendre sur une méthode. Que peut-on dire en attendant ?

La lecture publique ne fait pas partie des compétences obligatoires des départements. Aussi est-ce parfois un débat dans les milieux professionnels que de savoir si un conseil général pourrait supprimer sa BDP. Quoi qu'il en soit, aucun ne l'a fait ni, pour autant que nous le sachions, tenté de le faire. Aucun n'a laissé péricliter sa BDP dans des proportions telles qu'on puisse avoir le sentiment qu'il recherchait sa disparition.

\footnotetext{
(94) B. Calenge, "Les bibliothèques centrales de prêt: naissance de la lecture publique rurale", Histoire des bibliothèques françaises, t. 4 : Les bibliothèques au XXème siècle, 1914-1990, Paris, Promodis-Ed. du Cercle de la librairie, 1992, p. 312. (95) Ibid.

(96) Y. Gaillard, Rapport d'information fait au nom de la commission des Finances, du contrôle budgétaire et des comptes économiques de la Nation sur le rapport de la Cour des comptes relatif aux services déconcentrés du ministère de la Culture et de la Communication, annexe au procès-verbal de la séance du 10 juillet 2003, p. 13 et 34. Disponible sur le site du Sénat.
} 
Les BDP se sont informatisées, ouvertes aux disques et aux films. En 1986, la moyenne des emplois était de 15 par BDP ; elle était de 20 dix ans plus tard ${ }^{\left({ }^{97}\right.}$.

D'un autre côté, le niveau de développement des BDP est inégal. Des BDP végètent. Si des départements ont développé la leur, d'autres se sont contentés de la maintenir. Certaines de ces disparités sont héritées de la période antérieure à la décentralisation ; celle-ci les a parfois un peu atténuées, mais ne les a pas annulées.

\section{L’État aurait-il fait mieux que les départements?}

Selon un bilan provisoire dressé en $2000{ }^{988}$, si les moyens des BDP se sont nettement développés depuis 1980, c'est la période 1980-1985 - soit la période qui a précédé le transfert - qui a connu l'amélioration la plus sensible. Si les BCP n'avaient pas été décentralisées, l'État aurait-il poursuivi cet effort ?

En se fondant sur l'observation de la politique habituelle de celui-ci et singulièrement du ministère de la Culture, on risquera le pronostic rétrospectif suivant. Si elles étaient restées des services de l'État, les BDP se seraient, dans l'ensemble, ouvertes plus vite aux disques et aux films ; et leurs effectifs auraient moins augmenté.

S'agissant de l'informatisation, une opération conduite par l'État avant la décentralisation, considérée comme peu heureuse, porte à penser qu'il se serait saisi plus vite de la question que l'ensemble des départements, mais que l'issue n'aurait pas nécessairement été positive.

Le satisfecit décerné aux départements en matière de créations d'emploi est fondé. Pour autant, il n'est pas sans appeler des nuances ou à tout le moins des interrogations. Il apparaît en effet que "les personnels non spécifiques des bibliothèques ont augmenté encore plus vite que la moyenne" ${ }^{(99)}$.

Il y a deux façons d'interpréter cette donnée. La première est de considérer que des nonbibliothécaires sont venus opportunément renforcer l'équipe des bibliothécaires pour accomplir des tâches ne relevant pas de ceux-ci. Ne pourrait dans ce cas être porté au débit du département, le cas échéant, que de ne pas avoir assuré parallèlement la croissance des effectifs de bibliothécaires.

La seconde interprétation possible est de penser que des tâches bibliothéconomiques ont été confiées, pour diverses raisons, à des non-bibliothécaires, au détriment du professionnalisme du service.

Cette question en appelle une autre: celle des directions confiées à des nonbibliothécaires. Elle est trop complexe pour être traitée ici. En effet, pour diriger leur BDP, des conseils généraux préfèrent sciemment des non-bibliothécaires à des professionnels; pour autant que ce phénomène doive se traduire à plus ou moins long terme par une déperdition de la qualité du service, au moins sous certains aspects, ce qu'il permis de considérer comme probable, il faut le tenir pour une des conséquences négatives de la départementalisation des BDP.

Mais d'autres conseils généraux confient la direction de leur BDP à des non-professionnels faute de candidatures de professionnels ou de candidatures en nombre suffisant et/ou à tort ou à raison convaincantes. Ce qui est dès lors en cause c'est moins la départementalisation des BDP en tant que telle que l'insuffisance numérique du vivier, et donc en particulier le nombre des postes mis aux concours, l'attractivité comparée des départements, la formation des conservateurs, la mobilité et les moyens de la facilité, etc.

\footnotetext{
${ }^{(97)}$ A. Caraco, article cité, p. 16.

(98) Par Anne-Marie Bertrand: Évaluation BDP 2000. Résultats et synthèse. Document de travail, octobre 2000, dactylographié, document interne à la DLL.

${ }^{(99)}$ A. Caraco, article cité, p. 17.
} 


\section{$>$ Les effets de la départementalisation des BDP sur les politiques départementales}

Quand un bilan de la départementalisation des BDP est esquissé, il se limite généralement à l'examen des moyens de toute nature dont elles ont été - ou n'ont pas été - dotées.

Pour évaluer cette départementalisation, c'est un critère qui s'impose. Mais il en est d'autres. Ainsi, l'influence que la départementalisation des BDP a eu le cas échéant sur le développement d'une politique départementale en faveur des bibliothèques (le concours particulier départemental aidant), de la lecture, du livre, des TIC, etc.

S'agissant des bibliothèques, nombreux sont les conseils généraux qui ont mis en place un système d'aide à l'investissement (construction, équipement). Il s'y ajoute parfois une aide au fonctionnement (subventions pour le recrutement de personnels qualifiés, etc.). Selon toute vraisemblance, le transfert des BDP aux départements, en les dotant officiellement d'une compétence en matière de lecture publique, a favorisé la mise en place de tels dispositifs.

Mais encore ? Tous les départements ont-ils mis en place une politique de ce type ? Quels en sont, comparativement, les points forts et les points faibles selon les cas?

En matière d'attribution des aides, les conseils généraux appliquent des critères plus ou moins cohérents, plus ou moins exigeants, ils se réfèrent à une conception plus ou moins ambitieuse, plus ou moins rationnelle aussi de la desserte du territoire départemental. Là où sont mises en œuvre les politiques les plus pertinentes, quelle part la BDP a-t-elle prise dans cette orientation?

Il peut arriver qu'une politique départementale en faveur des bibliothèques, de la lecture, etc. soit conduite en dehors de la BDP (par exemple, elle n'instruit pas les demandes d'aide déposées auprès du département par les communes) voire, est-il permis de penser, à son détriment (financement parallèle de structures publiques, parapubliques ou privées chargées de missions analogues).

Il s'agit d'un phénomène complexe qui n'implique pas nécessairement que la départementalisation des BDP ni même celle des BDP concernées aient été en elles-mêmes inopportunes. Peuvent jouer, dans une telle situation, des facteurs aussi divers que l'histoire de la politique des départements, les rapports de force politiques et sociaux, le crédit personnel du directeur du service, tel qu'il peut d'ailleurs varier au fil du temps selon les évaluateurs, etc.

Quoi qu'il en soit, un des avantages que l'on pouvait attendre de la "départementalisation" des BDP, c'était la mise en place d'une politique globale en faveur des bibliothèques et de la lecture, la mise en cohérence de l'action de la BDP et des autres actions du département dans le même domaine - une mise en cohérence qu'aurait rendu plus difficile le fait que les BDP restent des services de l'État. II ne serait donc pas inutile de disposer d'un éclairage complet sur ce point.

\section{$>$ Les effets de la départementalisation des BDP sur leurs missions}

L'évolution des missions des BDP relève d'un exposé particulier.

Sur deux points, il serait tout particulièrement intéressant de mesurer les effets de leur départementalisation. Le premier est la desserte des établissements scolaires.

Les BDP se sont d'abord beaucoup appuyées sur eux, essentiellement les écoles primaires. Puis, elles se sont tendanciellement dégagées de ces liens. Des circulaires ministérielles les y ont incité $(1978,1985)$.

Or, les collèges dépendent des départements. Il y aurait donc lieu de s'interroger sur le point de savoir si les BDP, tout en desservant moins d'écoles, ne se sont pas mises à desservir plus de collèges. 
C'est ce que porte à penser la lecture des trois derniers Guides des BDP. Selon ces sources, en effet, les collèges représentent de 57 à 58 \% de l'ensemble des établissements (collèges, écoles maternelles et écoles élémentaires) desservis ${ }^{(100)}$.

Le second point est le développement d'une fonction de conservation dont on sait qu'elle n'était pas envisagée à l'origine, une fonction de conservation orientée vers les fonds ayant trait au département.

Selon les points de vue, l'implication croissante des BDP (si croissance il y a) dans ces deux missions apparaîtra comme une régression ou un progrès et sera portée à l'actif ou au passif de la départementalisation de ces services.

Cette départementalisation ayant eu lieu, le développement de ces deux missions était prévisible et, dès lors, le fait qu'elles aient ou non été développées pourra être lu comme mesurant le degré d'intégration des BDP dans les politiques départementales. Et donc la réussite de la "greffe".

\section{Risques et chances}

S'il fallait, aujourd'hui, caractériser la situation des BDP dans les départements, on pourrait dire que les BDP sont fortes parce qu'elles sont départementales. Et fragiles pour la même raison.

Communes, départements, régions : régulièrement, il est fait observer en France que le territoire national comporterait une subdivision territoriale de trop. Et le plus souvent, celle qui est dans la ligne de mire, c'est le département. Les BDP sont fragiles dans la mesure où la menace récurrente dont les départements font l'objet se concrétiserait. Toutefois, il est à noter que la disparition des départements ne signifierait pas nécessairement que des services du type des BDP ne leur succéderaient pas, après une redéfinition.

Parmi les compétences du département, s'est affirmée l'aide sociale. Il en résulte pour lui de lourdes charges, des charges dont le poids augmente en période de difficultés économiques.

Par ailleurs, les départements doivent aujourd'hui faire face à la montée en puissance, audessus d'eux, des régions, au-dessous, des groupements de communes.

Pour ces raisons, le département cherche à mieux identifier les missions qui lui reviendraient en propre. Celles qui apparaîtraient à tous (l'État, les communes, les régions) comme leur revenant de la façon la plus incontestable et sur lesquelles, obligés d'opérer des choix, ils pourraient concentrer leurs efforts.

Or, le développement de la lecture publique n'est pas sans présenter des chances sérieuses de s'affirmer comme une de ces missions.

En effet, en leur transférant les BDP, la loi a reconnu aux départements une compétence en la matière ; et une compétence que, à travers notamment les BDP, ils sont seuls à exercer de cette façon, à l'exclusion de la région. Par ailleurs, dans le cadre de l' "aménagement du territoire", objectif majeur des départements, les BM - que les BDP ont pour mission de développer et de soutenir - constituent un des équipements les plus consensuels. Pour peu qu'elles s'ouvrent aux NTIC et à la formation, elles font figure de services qui présentent un intérêt culturel mais aussi social et économique.

Comme on l'a vu, toutes les petites communes sont loin d'être dotées d'une bibliothèque, soit proprement municipale soit intercommunale. Et il faudra de nombreuses années pour que toutes bénéficient d'un tel équipement. De ce point de vue, les BDP restent utiles. Et le resteront longtemps.

\footnotetext{
(100) Mais tant le nombre des écoles que des collèges desservis diminue lentement. Écoles : 69 en 1999, 67 en 2000,64 en 2001. Collèges : 49 en 1999, 48 en 2000, 47 en 2001. Sources: Guide des BDP 2000 : données 1999; Guide des BDP 2002 [sic] : données 2000 ; Guide des BDP 2003 : données 2001.
} 
Mais elles le restent aussi là où, dans de petites communes, des bibliothèques municipales ou intercommunales ont vu le jour. Car le besoin se fait alors sentir de services coopératifs à même de leur apporter un appui dans maints domaines, à commencer par la formation. Ce chemin, les BDP s'y sont déjà engagées, qui, peu à peu, se transforment en "services départementaux de lecture publique" - abandonnant, çà et là, le mot "prêt" dans leur dénomination, et commençant à s'interroger sur le maintien du mot "bibliothèque". Dans cette logique, de plus en plus, la limite des 10000 habitants paraît artificielle.

Les bibliothèques départementales sont liées au département pour le meilleur et pour le pire. C'est un autre élément du bilan temporaire de leur décentralisation que l'on peut dresser. Il est juste d'ajouter que la même phrase aurait pu être prononcée si les BDP étaient restées des services de l'État.

b) Le concours particulier départemental

Le concours particulier départemental a évolué comme suit depuis 1992.

\begin{tabular}{|c|c|c|c|}
\hline Année & $\begin{array}{c}\text { Dépenses } \\
\text { (année précédente) }\end{array}$ & Répartis & $\begin{array}{c}\text { Taux } \\
\text { de concours } \\
\text { en } \%\end{array}$ \\
\hline 1992 & $75801407 \mathrm{~F}$ & $9299998 \mathrm{~F}$ & $12,26 \%$ \\
\hline 1993 & $91719977 \mathrm{~F}$ & $22639612 \mathrm{~F}$ & $24,68 \%$ \\
\hline 1994 & $90190815 \mathrm{~F}$ & $32495373 \mathrm{~F}$ & $36,03 \%$ \\
\hline 1995 & $73766077 \mathrm{~F}$ & $33188518 \mathrm{~F}$ & $44,99 \%$ \\
\hline 1996 & $79923269 \mathrm{~F}$ & $34306430 \mathrm{~F}$ & $42,92 \%$ \\
\hline 1997 & $77823761 \mathrm{~F}$ & $34719657 \mathrm{~F}$ & $44,61 \%$ \\
\hline 1998 & $80017086 \mathrm{~F}$ & $35256281 \mathrm{~F}$ & $44,06 \%$ \\
\hline 1999 & $110915981 \mathrm{~F}$ & $36236138 \mathrm{~F}$ & $32,67 \%$ \\
\hline 2000 & $114453903 \mathrm{~F}$ & $36533636 \mathrm{~F}$ & $31,92 \%$ \\
\hline 2001 & $120941169 \mathrm{~F}$ & $37784630 \mathrm{~F}$ & $31,24 \%$ \\
\hline 2002 & $140482932 \mathrm{~F}$ & $39321954 \mathrm{~F}$ & $27,99 \%$ \\
\hline 2003 & $135483101 \mathrm{~F}$ & $40224011 \mathrm{~F}$ & \\
\hline & $20654265 €$ & $6132111 €$ & $29,69 \%$ \\
\hline
\end{tabular}

Il a été indiqué plus haut que le budget affecté à l'origine par l'État au concours particulier départemental représentait 31 MF (50\% du budget d'investissement conservé jusqu'en 1992 par l'État au titre des BDP). Si cette somme n'est atteinte, actualisée, qu'en 1994, c'est parce que le transfert des crédits du ministère de la Culture au ministère de l'Intérieur s'est opéré progressivement "selon la règle de couverture des autorisations de programme par les crédits de paiement (30\% - $40 \%$ - $30 \%)$ " ${ }^{(101)}$.

Par rapport aux années 1995-1998, le taux de concours est aujourd'hui à la baisse. D'après les projets dont le ministère de la Culture est informé, en particulier en matière de constructions de nouvelles centrales pour les BDP elles-mêmes, cette tendance à la diminution a toute chance de s'accentuer dans les années à venir.

Pour évaluer les effets du dispositif, devrait être précisé le nombre des opérations qui ont été conduites avec son concours, qu'il s'agisse d'opérations concernant les BDP elles-mêmes ou d'opérations relatives aux BM concernées. Mais dans ce dernier cas et pour s'en tenir à lui, l'évaluation ne sera complète que si elle prend aussi en compte :

1) la qualité des opérations

Autrement dit, l'aide attribuée par le Conseil général obéit-elle ou non à des critères de desserte équilibrée et qualitative du territoire départemental ?

A ce propos, doit être relevé le fait suivant. Il a été indiqué que la même opération conduite au profit d'une BM pouvait bénéficier deux fois du concours particulier : directement, par le biais du concours particulier des communes ; indirectement, par le biais du concours particulier

(101) Ministère de l'Éducation nationale et de la Culture, ministère de l'Intérieur et de la Sécurité publique et ministère des Départements et Territoires d'outre-mer, circulaire du 17 mars 1993 aux préfets de région et de département et aux DRAC. 
départemental, aux termes duquel le département se verra rembourser une partie de la somme versée à la commune concernée.

Prenons le cas où l'opération en question ne satisferait pas aux critères permettant de bénéficier du concours particulier communal. Le département n'en émargera pas moins au concours particulier départemental. Par ce biais, l'État soutient donc une opération dont il estime simultanément qu'elle ne mérite pas d'être soutenue.

2) Comme dans le cas du concours particulier des BM (deuxième part), les financements de toute origine dont auront bénéficié, le cas échéant, les mêmes opérations.

Par ailleurs, supposons que des opérations conduites au profit de BM bénéficient de la part d'un département d'une aide s'ajoutant au montant qui lui sera remboursée au titre du concours particulier. L'attribution d'une telle aide pourra être considérée comme un des éventuels effets positifs induits du concours particulier, ou au contraire un de ses éventuels effets négatifs, selon que le régime des aides additionnelles obéira ou non aux critères déjà évoqués de desserte équilibrée et qualitative du territoire départemental.

\subsubsection{Nouvelles mesures: hypothèses}

\subsubsection{Le concours particulier et les conservateurs d'État}

Depuis plusieurs années, des questions sont régulièrement posées au sujet de l'aide que l'État apporte aux communes et aux départements pour leurs bibliothèques. modicité.

Ces questions tournent essentiellement autour de l'aide au fonctionnement des BM et de sa

Deux positions se font face.

a) La position des élus

Pour eux, l'État ne les aide pas suffisamment. Cette position est naturelle. Elle n'est pas idéologique, au contraire ; elle est pragmatique : tout financement permettant d'améliorer le fonctionnement des services de la commune sans grever le budget municipal est bon à prendre.

Si les élus pouvaient faire fonctionner les services communaux sans recourir à l'aide de l'État, ils s'y résoudraient sans peine. Dans le contexte actuel de la répartition des charges et des moyens, ils estiment qu'ils ne le peuvent difficilement. De surcroît, ils suspectent l'État de vouloir leur transférer des charges sans leur transférer aussi les moyens correspondants. Alors que la décentralisation pouvait apparaître comme une tendance politique lourde, approuvée par les principales formations politiques, toutes tendances confondues, n'a-t-on pas entendu des élus déclarer qu'elle avait désormais atteint les limites qu'il est souhaitable de lui assigner?

Les communes peinent à assurer leurs dépenses de fonctionnement : c'est dans cet esprit que des élus, sinon tous les élus, font observer que la première part du concours particulier se situe à un niveau anormalement bas.

Quelle solution ? Lors d'un colloque tenu en 1992, un représentant du comité des finances locales ${ }^{(102)}$ donne à ce sujet la position de celui-ci :

Ainsi, dans sa séance du 25 avril 1991, le comité a délivré un avis favorable au projet de décret fixant le taux de concours pour 1991, non sans avoir constaté la diminution de ce taux par rapport aux années précédentes et à la progression rapide des dépenses éligibles. Il a également souhaité dans sa délibération une

\footnotetext{
${ }^{(102)}$ Est consacré au comité des finances locales, l'article L 1211 du Code général des collectivités territoriales. Crée en 1979, ce comité, qui siège au ministère de l'Intérieur, associe des élus et des représentants de l'État ; il est présidé par un élu. Il a "pour objet de défendre les intérêts des collectivités locales sur le plan financier et d'harmoniser leur point de vue avec celui de l'État" (site du ministère). Il contrôle la répartition de la DGF. Le taux de la première part du concours particulier lui est soumis pour avis.
} 
révision du décret du 12 mars 1986, afin de mieux prendre en compte les besoins réels, et d'apporter une participation plus satisfaisante à la hausse des dépenses de fonctionnement.

Sa volonté de revoir les éléments structurels de ce mécanisme financier a été précisée l'année suivante, le 18 juin 1992. Le comité a adopté une délibération demandant que le taux de concours soit calculé à partir des dépenses réelles de fonctionnement effectuées par les communes, et non sur la base des dépenses prévisionnelles. Au cours du débat, les membres du comité avaient exprimé leur inquiétude de voir les charges augmenter, en raison de l'apparition de supports modernes de communication : sous l'effet du progrès technologique et de la demande des usagers, les bibliothèques se transforment de plus en plus en médiathèques.

Aussi, le comité avait estimé qu'une réforme de la dotation devait, soit intégrer dans la première part les crédits de la seconde part affectés à l'investissement, soit supprimer la spécialisation existante et inclure le concours particulier dans le tronc commun de la dotation générale de décentralisation. ${ }^{(103)}$

Donc: suppression du concours particulier. Ou de la deuxième part, les crédits venant abonder la première part.

Cette position était-elle véritablement représentative de celle des élus ? Si tous seraient évidemment d'accord pour déplorer la modicité de l'aide apportée au titre de la première part, il n'est pas sûr que tous approuveraient la disparition de la deuxième part.

Soutenue par le ministère des Finances, la position consistant à souhaiter la disparition de la deuxième part du concours particulier ne l'est pas par le ministère de l'Intérieur, au nom du nécessaire aménagement du territoire.

A juste titre, est-il permis de penser. Aux yeux des élus, une BM "moderne" tend aujourd'hui à passer pour un des équipements culturels de base, celui qu'une ville qui entend être réputée conduire une politique culturelle doit posséder. Et de ce point de vue, les bâtiments qui ont été construits depuis 25 ans jouent un rôle positif ; ils constituent une référence tangible, sont susceptibles de provoquer, provoquent un effet d'incitation, d'imitation.

Malgré tout, sans une aide financière de l'État, au moins une partie des projets ne verraient pas le jour, ou seraient réalisés sur un pied plus modeste, avec la perte d'attractivité et d'efficacité qui en résulterait.

Or justement, aux yeux de certains élus, le fait de s'abstenir de construire ou de voir moins grand constituerait dans certains cas un phénomène plutôt positif. En effet, l'aide apportée par l'État aux investissements à travers le concours particulier, combinée à l'obligation de satisfaire à des normes de surface, est considérée par eux comme un véritable "pousse-au-crime" : tout est fait pour pousser les villes à se doter d'équipements dont certaines peineront à assurer le fonctionnement.

A qui se trouve dans cet état d'esprit, il est naturel de souhaiter la disparition de la deuxième part du concours particulier.

Pour autant, l'argumentation développée n'est pas sans laisser place à des réponses. D'une part, les normes de surface dont l'État exige le respect pour que les villes puissent bénéficier de la deuxième part sont modérées - d'autant plus modérées qu'elles ont été fixées à une époque où le développement d'Internet et du multimédia, consommateurs d'espace, n'avait pas atteint le degré actuel. D'autre part, c'est bien aux villes qu'appartient la décision de construire ou de ne pas construire; se doter d'une bibliothèque efficace, ce n'est pas seulement l'édifier, c'est aussi développer des prestations ; et ceci va tellement de soi qu'on s'étonne de voir les élus être dépeints ou se dépeindre comme des êtres inconséquents, incapables de résister à l'attrait du fruit défendu.

Au cours des dernières années, la position défendue par les élus du comité des finances locales a plutôt été celle, plus tempérée que la position exprimée en 1991, d'une diminution du

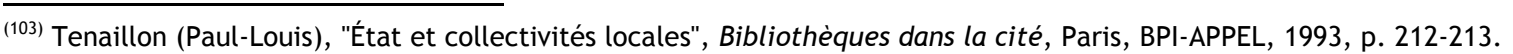


pourcentage de l'enveloppe du concours particulier consacré à la deuxième part (65\%) au profit de la première. Ainsi lors de la séance du 27 juin $2000{ }^{(104)}$.

b) La position du ministère de la Culture.

Comme lors des années qui virent lancer la décentralisation, le ministère est pris dans deux logiques contradictoires.

La première est dictée à la fois par un constat objectif (le développement des bibliothèques n'est pas achevé en France) et le réflexe institutionnel observable en pareil cas (ni l'État en général, ni chacun des ministères n'est pressé de se dessaisir de ses moyens d'action et donc par-là, pensent-ils, de s'affaiblir).

C'est cette logique qui a conduit le ministère de la Culture à se demander

- s'il n'y avait pas lieu d'augmenter la première part du concours particulier des BM, fût-ce - si l'on ne pouvait pas faire autrement - en lui transférant une partie de la seconde ;

- s'il ne conviendrait pas d'instituer un concours particulier pour le fonctionnement des $\mathrm{BDP}$, à l'instar de celui qui existe pour les BM.

La seconde logique est celle de la décentralisation.

Pour des raisons évidentes, cette logique n'est pas spontanément celle du ministère de la Culture. Mais il ne peut pas s'y soustraire.

C'est cette logique qui le conduit à se poser, à propos des BM, les questions suivantes :

$1^{\circ}$ ) Rembourser les frais de fonctionnement à hauteur de $3 \%$ a-t-il un sens ? Puisqu'on ne peut pas augmenter ce pourcentage, ne faut-il pas supprimer purement et simplement supprimer la première part du concours particulier?

$2^{\circ}$ ) L'État doit-il conserver la propriété des fonds d'État confiés à certaines BM ? ${ }^{(105)}$ D'une part, entre les fonds d'État et les fonds communaux, il est parfois difficile de faire la différence. D'autre part, tant l'État que les communes ne font pas la différence du point de vue des mesures qu'ils prennent (ou ne prennent pas) en leur faveur. Le transfert aux communes de la propriété des fonds d'État mettrait donc le droit en accord avec le fait.

De plus, si les villes se voyaient clairement reconnaître la pleine propriété de tous les fonds patrimoniaux qu'elles conservent, ne seraient-elles pas portées à se saisir davantage de leur préservation et de leur valorisation?

$3^{\circ}$ ) L'État doit-il continuer à mettre des conservateurs à la disposition de certaines BM (les $54 \mathrm{BMC})$ ? Dès lors que des bibliothèques qui ont des fonds patrimoniaux d'État n'ont pas de conservateurs d'État, et que les conservateurs d'État ne s'occupent pas que des fonds patrimoniaux, qu'ils appartiennent à l'État ou non, pourquoi ces 54 bibliothèques bénéficient-elles de conservateurs d'État plutôt que d'autres?

Si des conservateurs d'État continuent d'être mis à la disposition des villes, en fonction de quels critères ces villes doivent-elles être choisies, quelles doivent être les missions de ces conservateurs? Quelles sont les missions qui, dans les collectivités locales, justifient que l'État intervienne en prêtant son personnel ?

\footnotetext{
(104) Les comptes rendus des séances du comité des finances locales dont il est question dans ce texte sont disponibles sur le site du ministère de l'Intérieur, DGCL.

${ }^{(105)}$ Question posée en particulier par le rapport Béghain, ouvrage cité. De même, le 3º.
} 
Entre le tout et le rien, entre le maintien du système actuel, qui a un peu perdu en légitimité, et le retrait de l'État, des solutions intermédiaires sont parfois préconisées :

- S'agissant du concours particulier, maintien de la deuxième part (avec, le cas échéant, une sous-enveloppe qui, destinée à financer des gros équipements, prendrait la suite de la troisième part). Quant à l'aide au fonctionnement passant à l'heure actuelle par la première part, maintien du principe d'une aide, mais attribuée d'une façon plus sélective.

"Attribuée d'une façon plus sélective": cette expression est susceptible de désigner plusieurs cas de figure.

Premier cas de figure : maintien de la première part du concours particulier, c'est-à-dire du principe d'une aide permanente et automatique. Mais seraient favorisées, les bibliothèques jugées répondre à des critères de qualité plus exigeants que ce n'est actuellement le cas. En somme, le ministère de la culture s'efforcerait, ce faisant, de tirer tant bien que mal parti d'une situation qu'il déplore, de profiter d'une réduction du nombre des bénéficiaires - et/ou du niveau des aides jugée inéluctable pour tirer les bibliothèques vers le haut en donnant une prime à la qualité.

Deuxième cas de figure : suppression du principe de la permanence et de l'automaticité des aides, c'est-à-dire, en fait, de la première part du concours particulier. Mais conservation de tout ou partie de l'enveloppe - de la totalité de l'enveloppe, faut-il souhaiter - qui serait désormais attribuée de façon plus ou moins temporaire par le ministère de la Culture, via les DRAC, en fonction de ses priorités.

En somme, par un curieux phénomène de renversement, la première part du concours particulier redeviendrait, comme avant la décentralisation, un budget dont le ministère de la Culture aurait toute la maîtrise et qu'il distribuerait sous forme de subventions. On assisterait là, sous le rapport du fonctionnement, à un phénomène de même ordre que celui qui voit depuis quelques années la deuxième part du concours particulier être complétée par des crédits du titre VI.

Le comité des finances locales s'est récemment saisi de cette question. En effet, lors de la séance du 24 juin 2003, a été décidée, sur l'initiative du président ( $M$. Jean-Pierre Fourcade, maire et sénateur), la création d'un groupe de travail réunissant des élus du comité d'une part, des représentants de la DGCL et du ministère de la Culture d'autre part.

La piste - peut-on lire dans le compte rendu - est celle d'un "resserrement sensible de l'assiette". Il s'agit de "cibler sur des critères précis traduisant des actions prioritaires".

Il est à noter qu'en acceptant d'étudier cette hypothèse, les élus du comité ont adopté une position qu'on qualifiera d'ouverture. En effet, au cours de la même séance, le président du comité, dans la droite ligne d'une mise en cause constante de l'intangibilité de la deuxième part, avait commencé par se déclarer favorable à une réduction de celle-ci à $50 \%$ de l'enveloppe au profit de la première part, laquelle serait ainsi passée de 35 à $50 \%$. Le souhait en avait déjà été formulé en 2001 : selon le compte rendu de la séance du 12 juillet, "les membres du comité ont souhaité un rééquilibrage, à hauteur minimum de la moitié, du concours particulier au profit de la part consacrée aux dépenses de fonctionnement des BM" - "en regrettant", est-il ajouté, "que les recommandations faites depuis plusieurs années en ce sens n'aient pas été suivies d'effet" ; ce jour là, la mauvaise humeur des élus à cet égard les conduit à donner un avis réservé sur le taux de concours qui leur est, comme à l'habitude, soumis.

Le 24 juin 2003, c'est sur la suggestion du directeur général des collectivités locales qu'est plutôt suivie la piste d'une réduction de l'assiette - du DGCL qui avait indiqué que l'affectation de $50 \%$ du budget global du concours particulier à la première part n'aurait fait passer le taux de concours qu'à $4,35 \%$ au lieu de 3,29.

Il était dans l'ordre des choses que fût envisagé un recentrement de l'aide attribuée au titre de la première part dès lors que le montant de celle-ci ne parvenait pas à suivre l'augmentation du nombre des bibliothèques et de leurs dépenses.

Pour autant, un tel recentrement ne serait pas sans soulever des questions. Ainsi, comment les villes qui verront disparaître ou diminuer l'aide au fonctionnement qui leur est attribuée réagiront-elles ? Sera-ce nécessairement en compensant ce "manque à gagner" ? Au bout du compte, le budget des bibliothèques ne se trouvera-t-il pas diminué d'autant, avec les conséquences que cela implique sur la qualité des prestations? 
Il est à signaler que selon certains scénarios, le maintien de la deuxième part du concours particulier pourrait s'accompagner de sa fusion avec le concours particulier départemental.

- S'agissant de la mise à disposition de conservateurs d'État, maintien du principe, mais application différente (élargissement du nombre des BM bénéficiaires potentielles, mise à disposition pour des missions précisées limitativement dans le temps ou quant à la nature des missions, etc.).

En bref, de la part de l'État, la conception qui tend à prévaloir, ainsi qu'il était prévisible, est la suivante.

* Au nom de l'aménagement du territoire, l'État est fondé à aider communes et départements à financer leurs investissements.

Ainsi la notion d'aménagement du territoire milite-t-elle pour le maintien de la deuxième part du concours particulier des BM et du concours particulier départemental.

Poussée au bout de sa logique, qui est égalitaire, elle fait en outre un devoir à l'État non seulement d'accompagner la volonté des collectivités locales de réaliser des investissements mais de les inciter à en faire là où il estime que des besoins se font sentir. C'est de cette conception que relève l'apparition au cours des années récentes, aux côtés de la deuxième part du concours particulier et pour aider le même type d'opérations, de crédits d'investissement gérés directement par le ministère de la culture (dotation exceptionnelle de 2000 et 2001, programme Les ruches). Dans le même ordre d'idées, il doit être relevé que les BM ont plusieurs fois bénéficié depuis les années 1990 de budgets alloués au titre de l'aménagement du territoire. concernées.

* Le fonctionnement des bibliothèques territoriales relève des collectivités locales

* Il n'est pas inenvisageable que l'État fasse des exceptions à la règle selon laquelle le fonctionnement des bibliothèques territoriales relève des collectivités locales concernées. Mais ce doit être, dans ce cas, en fonction de critères justifiant pleinement qu'il intervienne, lui, l'État, garant de l'intérêt national.

Qu'est-ce qui est à l'œuvre ici, sinon l'application au domaine culturel - une application jusque là freinée, différée - de la philosophie de la décentralisation ?

\subsubsection{Une alternative à l'État parisien : l'échelon régional}

Sous le rapport des bibliothèques comme dans d'autres domaines, culturels ou non, qu'il s'agisse pour l'État - tel qu'il est représenté par l'administration centrale du ministère de la Culture - de poursuivre la déconcentration, de décentraliser des dispositifs existants ou de rechercher pour les bibliothèques auprès des collectivités locales des financements additionnels ou substitutifs, l'échelon qui, à l'heure actuelle, semble retenir de façon privilégiée son attention est l'échelon régional.

A l'origine de l'intérêt que celui-ci suscite de la part de l'État parisien, se trouve selon les cas la recherche d'une efficacité accrue de ses services (déconcentration) ou des services publics en général par le transfert ou l'assignation de responsabilités à une collectivité locale à la fois plus proche des villes et dotée d'une solide assise géographique, politique et financière. Ou les deux.

\section{a) Poursuivre la déconcentration}

Le cas échéant, c'est-à-dire là où existent des FRAB, une partie des crédits consacrés à la restauration et à la conservation par la DLL est d'ores et déjà déconcentrée. Il est question de poursuivre cette déconcentration, ainsi que de substituer au CNSPB des conseils régionaux. Cette substitution serait cohérente avec le fait que, à compter du $1^{\text {er }}$ janvier 1998, le préfet de région a été substitué au ministre à titre de représentant de l'État habilité à émettre l'avis de règle sur les restaurations de documents patrimoniaux projetées par les BM ${ }^{(106)}$.

\footnotetext{
(106) Décret n 97-1201 du 24 décembre 1997 (journal officiel du 27).
} 
Cette éventuelle mesure ne semble pas devoir appeler d'objection, pour peu que chacun des conseils régionaux qui succéderont au Conseil national puisse bénéficier de toutes les compétences nécessaires. Ces compétences incluent l'indépendance à l'égard des intérêts locaux.

b) Décentraliser des dispositifs existants

Il est parfois question d'une "décentralisation" du concours particulier des communes (107), option qui comporte plusieurs sous-options. Ainsi, dans le cas de la deuxième part, les régions s'en verraient-elles remettre la gestion, soit que les crédits se fondent dans leur budget, soit qu'ils continuent à ne pouvoir être utilisés que pour les bibliothèques.

La première hypothèse (fusion des crédits du concours particulier dans le budget des conseils régionaux) n'est évidemment pas sans risque.

Certes, des régions, intéressées par le développement des bibliothèques, ne manqueraient pas d'abonder l'enveloppe que l'État leur aurait transférée. Tout comme des départements ont assuré le développement de la BDP après qu'elle leur a été transférée.

Mais d'autres régions pourraient décider d'amputer cette enveloppe voire de mettre fin à ce type d'aide. De ce point de vue, la situation ne serait pas tout à fait comparable à celle des départements, dont la lecture publique est une des compétences légales explicites.

Dans ces conditions, en cas de décentralisation de l'enveloppe du concours particulier (deuxième part) auprès des régions, la "sanctuarisation" de cette enveloppe au sein des budgets régionaux présenterait l'avantage de protéger les crédits. ${ }^{(108)}$

Utilisable pour les BDP mais aussi pour les BM aidées par le conseil général, le concours particulier départemental présente un exemple de ce que pourrait être un concours particulier pour l'investissement régionalisé. Or, quels enseignements peut-on tirer du dispositif du concours particulier départemental?

Au chapitre du positif, il a incité les conseils généraux à financer des investissements. Autrement dit, il a drainé des crédits qui sont venus s'ajouter à ceux de l'enveloppe du concours lui-même. Au chapitre du négatif, il a pu être utilisé pour des opérations n'obéissant pas à des critères de desserte équilibrée et qualitative du territoire.

Un concours particulier pour l'investissement décentralisé efficace est donc un concours particulier qui reste tel, c'est-à-dire dont les crédits sont fléchés, et qui obéit à des critères qualitatifs. Sous ces réserves, il est sans doute d'autant plus efficace qu'il est décentralisé, dans la mesure où cette décentralisation draine des crédits additionnels.

A la place des régions, sont quelquefois cités, comme bénéficiaires d'une décentralisation du concours particulier des BM, les départements.

Une chose est sûre : il semblerait peu cohérent que ces deux collectivités disposent d'un concours particulier pour l'investissement au profit des BM, même s'il ne s'agit pas des mêmes catégories de BM. Une décentralisation du concours particulier des BM (deuxième part) conduirait donc nécessairement à poser la question de sa fusion avec le concours particulier départemental.

c) La recherche de financements additionnels ou substitutifs

D'ores et déjà, les conseils régionaux concourent aux investissements effectués en faveur des bibliothèques :

\footnotetext{
(107) Ainsi, sans autre précision, dans le rapport Latarjet (ouvrage cité, p. 24) qui se fait l'écho des "personnes interrogées". Les témoignages recueillis, indique l'avant-propos, émanent d'élus, de chefs d'entreprise, de représentants de l'État et de dirigeants d'établissements culturels.

(108) Pour une opposition à la décentralisation du concours particulier, en particulier parce qu'elle serait susceptible de s'accompagner d’un "défléchage" : fédération des Sgen-CFDT, "Bibliothèques : plus de décentralisation ?", 20 déc. 2002, site de la fédération.
} 
- Aux collectivités concernées, ils attribuent, le cas échéant, des subventions pour la construction et l'équipement. Les bénéficiaires peuvent être des bibliothèques universitaires, mais aussi des bibliothèques territoriales.

- Dans plusieurs régions, ils alimentent, aux côtés du ministère de la Culture, un fonds destiné à acquérir des documents patrimoniaux pour les $B M$. C'est le cas dans les régions suivantes, citées dans l'ordre des créations des fonds (FRAB) : Bretagne, Bourgogne et Pays-de-la-Loire (1990) ; Champagne-Ardenne (1992) ; Aquitaine et Rhône-Alpes (1993) ; Centre, Haute-Normandie et Lorraine (1994) ; Auvergne (1999); Midi-Pyrénées (2000). Parfois, ce fonds est aussi destiné à financer la restauration de documents. ${ }^{(109)}$

Ce qui semble bien à l'ordre du jour, c'est manifestement d'intéresser les régions au fonctionnement des bibliothèques. Sinon directement (aide à chacune d'elle), du moins indirectement (développement des services et actions coopératifs).

Il serait plus exact de dire : de les y intéresser davantage. En effet, dans les régions où existe une agence de coopération compétente pour les bibliothèques, c'est-à-dire dans presque toutes ${ }^{(110)}$, le conseil régional participe à son financement, même si c'est de façon plus ou moins importante selon les cas ${ }^{(111)}$; pour indirecte qu'elle soit, c'est bien d'une aide au fonctionnement qu'il s'agit.

De même touche-t-on au fonctionnement quand des FRRAB élargissent à la valorisation, ainsi qu'il arrive, les subventions qu'ils allouent au titre du patrimoine. Ou quand une région, conjoignant l'intérêt porté par les conseils régionaux au patrimoine d'une part et au TIC d'autre part, co-finance avec l'État la constitution d'une "banque numérique du savoir", faite de fonds d'archives et de fonds patrimoniaux de bibliothèques numérisés ${ }^{(112)}$.

Au moment où ces lignes sont écrites, une disposition réglementaire est en projet, permettant aux régions de recruter, comme les départements et les villes, des conservateurs territoriaux des bibliothèques.

De cette intention manifeste d'impliquer davantage les régions dans le fonctionnement des bibliothèques, les professionnels ne se plaindront pas.

En effet, il y a au moins 70 ans qu'ils signalent l'intérêt pour ne pas dire la nécessité qu'il y aurait à disposer pour effectuer certaines tâches profitables aux bibliothèques, entre un ensemble donné de communes mais aussi de départements, et Paris, d'un échelon intermédiaire, un échelon "régional" (l'acception de ce mot a varié) ${ }^{(113)}$.

Ainsi, pour les créateurs des BCP, celles-ci ne devaient constituer que l'élément "terminal", le service de proximité d'un dispositif qui comprenait en outre, d'une part, 9 centres régionaux, d'autre part, une bibliothèque nationale de prêt ${ }^{(14)}$.

C'est dans la continuité de ce "pro-régionalisme" que s'inscrivent les rédacteurs du décret bien connu, dit sur le contrôle technique $(1988)^{(115)}$, quand, stipulant que les dispositions du texte s'appliquent aux régions, ils envisagent que des bibliothèques régionales puissent un jour exister.

\footnotetext{
(109) Ne se limitent pas à une aide aux acquisitions, les fonds (FRRAB) des régions Auvergne, Bretagne, Centre et MidiPyrénées.

${ }^{(110)} \mathrm{Au}$ moment où ces lignes sont écrites (février 2004) avec le concours de la FFCB (Béatrice Pedot), les seules régions qui ne comportent pas d'agence en métropole sont l'île-de-France (qui n'en a jamais eu) et Pays-de-la-Loire (qui en a une jusqu'en 1997). On inclut dans les régions qui possèdent une agence la Lorraine, dont l'action en la matière ne passe pas véritablement par une agence mais par un service du conseil régional. La Corse n'a pas d'agence. Outre-Mer, une agence a été créée en Guadeloupe en 2001.

(111) Selon une enquête conduite en 2001 par la FFCB, et dont les résultats sont vraisemblablement toujours valables, seuls les conseils régionaux de l'Alsace et du Nord-pas-de-Calais ne participaient pas au financement de l'agence. S'agissant du Languedoc-Roussillon, l'information n'avait pas été communiquée. Renseignements pris, le conseil régional de cette région a participé, pour la première fois depuis des années, au financement de l'agence en 2003.

${ }^{(112)}$ Il s'agit de la banque numérique du savoir aquitain (BNSA), inscrite au contrat de plan État-région 2000-2006.

${ }^{(113)}$ Voir notamment J. Goasguen, "Une expérience oubliée de bibliothèques régionales (1963-1965)", Le livre et l'art. Études offertes en hommage à Pierre Lelièvre, Paris, Somogy, et Villeurbanne, enssib, 2000, p. 91-104.

(114) B. Calenge, chapitre cité, p. 299.
} 
Depuis le moment où dans les années $1930 \mathrm{P}$. Lelièvre préconisait la formation de bibliothèques interdépartementales ou régionales et même depuis les années 1980, et aujourd'hui, les raisons de juger opportune l'existence d'un tel échelon régional ont pour partie changé. Mais il en est toujours.

Or, au souhait qu'on pourrait dire professionnel, technique, des bibliothécaires, la transformation de la région en collectivité territoriale et son affirmation croissante depuis les années 1980 ont fourni une chance accrue de se réaliser. En effet, la région, c'est désormais, pour un territoire précis, un ensemble de moyens et de services bien identifiés.

En somme, entre les bibliothèques et les régions, il reste à organiser en ce début du XXI ème siècle la même rencontre que, dans les années 1980, entre les bibliothèques et les départements.

C'est un rendez-vous manqué de ce point de vue, au moins jusqu'à présent, que l'édification des bibliothèques municipales à vocation régionale, puisque, dans cette dénomination, "régionale" doit être compris comme "extra-municipal". Dès lors, il était loisible aux conseils régionaux d'estimer que le fonctionnement de ces bibliothèques ne le concernait pas.

\subsubsection{Pour aider à la réflexion}

Il n'est ni possible ni opportun de procéder ici à une évaluation complète de la politique de l'État à l'égard des bibliothèques territoriales depuis 25 ans. Et pas davantage de lui en assigner une pour les années à venir. On se limitera aux observations suivantes.

\subsubsection{A propos du conseil et de l'évaluation}

Quand ils nourrissent des projets, villes et départements sont demandeurs d'une expertise qu'ils reconnaissent aux services de l'État. Cette demande est ambiguë : les collectivités locales, à la fois, sont dans l'attente de préconisations, et veulent être libres de ne pas s'y conformer.

Pour les suivre ou ne pas les suivre, il reste que villes et départements sont preneurs de conseils. Or, les conditions optimales ne sont actuellement pas réunies pour que ces conseils puissent être délivrés.

Ainsi, "guide technique et réglementaire", Bibliothèques dans la cité a-t-il huit ans (1996) plus si l'on se souvient que ce livre fut mis en chantier plusieurs années avant de paraître. Il est à remplacer. Un sujet, en particulier, doit désormais être traité : celui des procédures et schémas d'organisation à adopter en cas de mutualisation d'une ou de plusieurs bibliothèques par des communes.

La crainte ressentie par l'État à l'idée qu'on pourrait le suspecter d'édicter des normes ne saurait aller jusqu'à le retenir de formuler, au nom de l'intérêt public, des recommandations.

Elles seront d'autant plus pertinentes que la recherche aura été mise à contribution. Or, s'agissant de la bibliothèque en tant que stratégie complexe, matérielle et intellectuelle, de mise à disposition de collections, d'informations et de services, cette recherche est presque inexistante. $\mathrm{Ne}$ saurait être considérés comme satisfaisants de ce point de vue, en effet, ni des publications professionnelles utiles mais souvent peu novatrices, ni des travaux qui, axés soit sur l'histoire du livre soir sur le document numérique, méconnaissent la bibliothèque comme incluant ces sujets mais les dépassant

Une doctrine se développe, selon laquelle les conseillers, dans les DRAC, doivent tendre à une polyvalence accrue. D'une telle évolution, on saisit l'intérêt. Mais, d'un autre côté, l'éloignement par rapport à la spécialisation risque de s'effectuer au détriment de la compétence cette compétence, que, justement, les collectivités locales attendent des services de l'État.

\footnotetext{
(115) Aujourd'hui codifié dans la partie réglementaire du Code général des collectivités territoriales. Il n'est pas indifférent qu'un des rédacteurs de ce décret, J. Goasguen, ait participé sous l'autorité de Pierre Lelièvre à un essai de mise en place de bibliothèques interdépartementales.
} 
Cette compétence passe par la possession des connaissances appropriées. On pense ici aux fonds patrimoniaux, mais également à celles que nécessite la conception d'un équipement.

Elle passe aussi par le nombre des agents. En effet, à champ d'intervention égal, moins les conseillers seront nombreux, plus nombreux seront les domaines que chacun d'eux devra traiter.

Il a été fait allusion au fait que l'État avait multiplié les dispositifs contractuels avec les collectivités locales. A juste titre, il éprouve à présent le besoin de mettre de l'ordre dans ce paysage en substituant, dans ses négociations, une approche globale à des approches sectorielles. Or, cette démarche nouvelle, dont la pertinence ne fait aucun doute, ne nécessite pas que les conseillers poussent la polyvalence à l'extrême mais que, sous l'autorité du DRAC, ils travaillent ensemble et non concurremment.

"La logique paradoxale de la décentralisation", déclare à juste titre le ministre de la Culture ${ }^{(116)}$, "c'est qu'il faut que les services de l'État déconcentrés soient mieux armés pour constituer, pour les collectivités locales, des interlocuteurs avertis, compétents, efficaces et rapides. [...] Il m'est arrivé de me demander si pour certaines fonctions [...] on ne pouvait pas mutualiser les effectifs entre deux Directions régionales [...]. Cela me semble possible mais le gain sera en fin de compte très faible. La vérité, c'est qu'il faut, dans les domaines où l'État encadre, où l'État contrôle et partout où l'État exécute, que les services soient suffisamment dotés".

Si le conseil ne peut plus être fourni par les DRAC, vers qui villes et départements se tourneront-ils pour l'obtenir? Vers des prestataires privés? Leurs prestations sont inégales, et souvent très coûteuses.

Vers l'Inspection générale des bibliothèques? Il lui arrive d'ores et déjà d'intervenir en amont. Davantage, ses huit membres n'y suffiraient pas. Supposons toutefois que la possibilité se présente de doubler cet effectif. Passons sur le paradoxe que représenterait le fait de réinvestir l'administration centrale - l'administration centrale dont l'Inspection fait partie, même si elle ne se confond pas avec les bureaux - de missions qu'il s'est justement agi depuis 25 ans de transférer aux DRAC. Les raisons qui ont conduit à opérer ce transfert demeurent : s'agissant du conseil, les DRAC, plus proches du terrain, sont plus efficaces que les bureaux parisiens. Par ailleurs, l'Inspection est chargée du contrôle et l'évaluation, qui sont et seront plus que jamais nécessaires. Or, elle ne peut pas intervenir au-delà d'un certain point dans l'amont des projets sans devenir juge et partie.

Force est de se rendre à cette évidence : s'il est vrai que la décentralisation, une décentralisation accrue, reste nécessaire, et qu'elle appelle complémentairement une déconcentration des services de l'État, elle ne relève pas forcément pour celui-ci d'un processus d'économies programmées ; même proche des collectivités locales, surtout proche des collectivités locales, le fonctionnement optimal des services de l'État a un coût.

En matière d'évaluation, des progrès restent à faire quant aux critères (de ce point de vue aussi, l'actualisation des recommandations est nécessaire) et aux moyens (consolidation de l'Inspection générale des bibliothèques, interrogation sur son champ d'action et ses méthodes).

\subsubsection{Le patrimoine}

Concernant les bibliothèques, avant les années 1980, il n'était de "patrimoine" qu'à la Bibliothèque nationale. L'idée que les BM elles aussi - et pas seulement les BMC - conservent des fonds précieux à divers titres s'est affirmée. L'action du ministère de la Culture y est pour beaucoup.

Sachant que dans ce domaine, l'État estime avoir des responsabilités particulières, les moyens qu'ils dégagent sont-ils à la hauteur des ambitions?

Il convient d'ajouter que, au moment où ces lignes sont écrites (mars 2004), la DLL prépare un "plan d'action pour le patrimoine écrit".

(116) Y. Gaillard, rapport cité, p. 26-27. 


\subsubsection{Les réseaux}

Ainsi que les professionnels le savent, en matière de bibliothèques, la constitution de réseaux est utile voire indispensable. Il s'agit notamment : de se répartir, dans certaines limites, acquisitions et conservation; de mutualiser le signalement des documents; de permettre la circulation des documents ; de se rendre des services ; de faciliter la communication réciproque des informations utiles de toute nature ; de réaliser des économies d'échelle.

Les réseaux peuvent être nationaux. Ils peuvent être aussi locaux, cet adjectif étant luimême susceptible de se décliner: communaux, intercommunaux, départementaux, interdépartementaux, régionaux, interrégionaux.

En outre, la mise en place d'une coopération internationale ne passe plus nécessairement par les capitales des états concernés. Elle peut réunir, par-delà les frontières, des collectivités locales qui auront établi directement des liens.

Il paraît clair que l'État est le mieux à même de mettre en place les réseaux nationaux. II dispose directement pour ce faire de quelques bibliothèques. $\mathrm{Si}$, pour gagner du temps, on recourt pour caractériser de ce point de vue la situation aux types d'annotations qui figurent sur les livrets scolaires, au risque de la caricature et de l'injustice, on dira :

- Bibliothèque nationale de France : sur la voie consistant, pour cet établissement, à mériter pleinement le qualificatif de national en oeuvrant au bénéfice de l'ensemble du territoire et pas seulement de Paris, des progrès notables - à consolider et à amplifier.

$\mathrm{Au}$ titre du programme qu'on souhaitera voir fixer à la BnF dans cette perspective, mentionnons le signalement dans le CCF - dont la réalisation lui a été confiée - des fonds patrimoniaux de diverses BM. On veut parler de celles - parfois importantes- qui, pour diverses raisons, n'ont pu bénéficier de la campagne de rétroconversion des catalogues concernés conduite il y a quelques années.

- Bibliothèque publique d'information : ne doit pas se contenter d'être un exemple pour ses camarades plus faibles, doit aussi leur prêter main forte. Sous ce rapport : une incontestable bonne volonté.

- La Joie par les livres : les services rendus aux bibliothèques territoriales ne sont pas à la hauteur des talents de cet établissement spécialisé prestigieux. La part doit être faite, dans ce diagnostic, d'une insuffisance de moyens particulièrement patente.

- Médiathèque de la Cité des sciences et de l'industrie : aux bibliothèques territoriales, cette bibliothèque rend moins de services qu'elle ne leur en a rendus naguère. Il ne fait pourtant aucun doute que, dans le domaine qui est le sien, elle ne soit appelée à jouer un rôle national, au même titre que les trois autres bibliothèques citées précédemment. Dû à divers facteurs, le relatif retrait qu'on a pu voir la médiathèque de la Villette opérer au fil des années est d'autant plus paradoxal que la diffusion de la culture scientifique et technique fait régulièrement l'objet de "relances politiques". Et que les déficiences des bibliothèques publiques en la matière ne laissent pas d'être rappelées.

Précisément, annoncé par les ministres chargés de la culture et de la recherche (février 2004), un train de mesures en faveur du développement de la culture scientifique et technique devrait rendre caduque ce portrait d'une médiathèque de la Villette en deçà d'elle-même. En effet, le plan en question lui assigne un rôle retrouvé et accru de fournisseur de services et de conseils aux bibliothèques publiques.

L'existence de réseaux nationaux dont la tête est à Paris ne signifie pas qu'ils ne doivent pas évoluer vers des relations moins hiérarchiques et plus partenariales, moins verticales et plus horizontales. 
Il est permis de penser que la mise en place et le fonctionnement de réseaux locaux relèvent, eux, des collectivités locales concernées. Or, l'expérience montre que cette préoccupation ne leur est pas spontanée.

Si la tentation de l'État est de faire passer les particularités locales sous la toise unique d'une seule règle, inversement, celle des collectivités locales est d'ignorer ce qui est au-delà de leurs propres limites.

Il apparaît donc que l'intervention de l'État n'est pas superflue en la matière. Au demeurant, la frontière entre réseaux locaux et réseaux nationaux n'est pas toujours tranchée. Ils présentent des points d'intersection naturels voire nécessaires.

Le respect de l'autonomie des collectivités locales ne saurait empêcher l'État de nourrir et d'émettre des idées, certes, d'une part, sur le rôle respectif de l'État et des collectivités locales, mais d'autre part, sur le rôle respectif des différents types de collectivités locales.

Ainsi que l'a fait récemment remarquer le ministre de la Culture, les deux questions sont liées : "car comment voulez-vous", demande-t-il en 2003 devant une commission sénatoriale, "que l'État clarifie sa relation avec les collectivités locales quand celles-ci n'ont d'une part aucune obligation de mettre en ceuvre des actions ou des initiatives culturelles, et que, d'autre part, ces initiatives, elles les exercent dans un dispositif et selon des modalités extrêmement désordonnées ?" (117)

Selon toute vraisemblance, la question est en fait plus complexe qu'il n'y paraît.

D'un côté, l'État peut, doit désigner les types de collectivités locales qui lui paraissent le mieux à même de remplir certaines missions coopératives. C'est ce qu'il a fait - non sans succès, même s'il ne faut pas l'exagérer - en confiant aux départements le service public de la lecture en milieu rural. D'un autre côté, il ne doit pas brider les initiatives, si, tout bien pesé, elles permettent d'atteindre les buts poursuivis.

Ainsi, déterminer quelles collectivités locales, dans une région, sont appelées à faire quoi, doit-il dépendre selon les cas et simultanément de cadres et d'orientations nationaux, énoncés à Paris, et de la prise en compte, dans chaque région, du contexte local. Cette prise en compte, c'est aux DRAC qu'elle revient; si la déconcentration a un sens, c'est bien, en particulier, pour permettre cette adaptation des stratégies nationales aux terrains.

BM petites et moyennes. Grosses BM jouant un rôle coopératif de fait. BMVR. Bibliothèques intercommunales. BDP, et réseaux des BDP - parfois partiellement inclus dans un autre ensemble coopératif (structure intercommunale). Agences de coopération. Régions. Sans oublier les universités, dont les bibliothèques ont poursuivi leur développement, essaimant pour certaines d'entre elles dans des antennes délocalisées. Et des établissements scolaires dont les bibliothèques se sont elles aussi développées et ressemblent parfois, à s'y méprendre, à des bibliothèques de lecture publique. Pour partie, le paysage des acteurs potentiels de la coopération ressemble à celui d'hier ; pour une autre part, il est plus complexe : les acteurs en question sont plus nombreux, plus divers et les rôles respectifs sont répartis de façon moins claire et moins contrastée ${ }^{(118)}$. Il s'agit donc ici d'identifier, sans a priori dogmatique, qui est le mieux à même de faire quoi.

Entre une région plus proche des villes concernées que Paris, mais malgré tout encore un peu lointaine pour qu'on puisse en attendre certains types de services, et de grosses bibliothèques municipales à vocation coopérative dont l'ombre est parfois perçue comme menaçante (en somme, trop proches), il se pourrait que le département conservât ses chances. Même auprès des communes de plus de 10000 habitants.

\footnotetext{
(117) Y. Gaillard, rapport cité, p. 16.

(118) Bon exposé de l'évolution du paysage des bibliothèques et du contexte, et des défis à relever par N. Galaud, "Bibliothèques et territoires", BBF, t. 46, 2001, n 3 , p. 20-27.
} 
A tous les partenaires, État compris, la récente invention des établissements publics de coopération culturelle $\left(2002{ }^{(119)}\right)$ fournit un lieu approprié pour agir de concert.

\subsubsection{Les personnels}

\section{$>$ L'homologie des deux fonctions publiques}

Un principe veut que la fonction publique d'État et la fonction publique territoriale soient homologues. Autrement dit, que les corps et les cadres d'emploi (on parle de corps pour la fonction publique d'État, mais de cadres d'emploi pour la fonction publique territoriale) se correspondent. Il s'agit d'équité. Il s'agit aussi de permettre la circulation des deux familles de fonctionnaires entre les deux fonctions publiques, ceci afin d'élargir les possibilités offertes aux personnels mais aussi de faciliter le dialogue entre les deux univers et d'enrichir le cas échéant chacun des pratiques de l'autre.

Or, dans le cas des bibliothèques, ce principe d'homologie connaît une exception notable. Et aux conséquences négatives.

En effet, dans la fonction publique d'État, au-dessus du corps des conservateurs proprement dits, dont le grade terminal est conservateur en chef, il existe un corps de "conservateurs généraux". Or, ce corps n'a pas d'équivalent parmi les cadres d'emplois de la fonction publique territoriale ${ }^{\left({ }^{120}\right)}$. Il en résulte, d'une part, que les conservateurs généraux de l'État ne peuvent pas être détachés sur des postes territoriaux, d'autre part, que les conservateurs d'État qui, détachés sur un poste territorial, veulent accéder au généralat doivent réintégrer les bibliothèques de l'État ${ }^{(121)}$.

En outre, régulièrement, des conservateurs territoriaux demandent à être détachés dans des bibliothèques de l'État, tandis que le mouvement est moindre dans l'autre sens, et, le moment venu, ne retournent pas dans la fonction publique territoriale mais demandent à être intégrés dans la fonction publique d'État. Plusieurs raisons les y portent, qui se limitent pas à l'absence de cadre d'emploi de conservateur général territorial ; mais cette absence fait partie de ces raisons. Elle contribue donc à affaiblir la filière concernée de la fonction publique territoriale.

Outre les villes et équipements d'une certaine importance, un phénomène en expansion vient à l'appui des plaidoyers anciens pour la création d'un cadre d'emploi de conservateur général territorial. On veut parler de l'intercommunalité, en tant qu'elle conduit à créer des ensembles d'équipements plus vastes que ceux d'une ville unique.

\section{$>$ Le professionnalisme}

Être bibliothécaire exige une qualification spécialisée, garante de la qualité du service rendu. Cette idée est aujourd'hui assez largement répandue dans les milieux politico-administratifs tant locaux que nationaux. Mais elle est loin de l'être partout. Elle a un vieil adversaire, qui a cédé du terrain mais n'a pas battu en retraite: l'amateurisme", représenté en particulier par le bénévolat - il s'agit de la conviction, nourrie de méconnaissance, que pour faire fonctionner une bibliothèque, il suffit d'aimer "les livres".

Or, le "professionnalisme" s'est peu à peu découvert un autre ennemi : la polyvalence. Pour diverses raisons que ce n'est pas le lieu d'exposer, la tendance ne semble pas être, dans les collectivités locales, à adhérer sans retour au maintien de filières culturelles séparées.

Cette attitude à l'égard de la spécialisation des filières a pour intérêt de rappeler à chacune d'elles, d'une part, que les connaissances spécialisées qui la caractérisent ne sont pas exclusives de

\footnotetext{
(119) Loi $n^{\circ} 2002-6$ du 4 janvier 2002 (Journal officiel du 5).

(120) Pas plus dans le cas des conservateurs du patrimoine que dans celui des conservateurs des bibliothèques.

(121) Ainsi que, le cas échéant, les BM qui ne sont pas les BMC. Il est à signaler que, pour la même raison, des conservateurs territoriaux rejoignent régulièrement la fonction publique de l'État, sans que leurs départs soient compensés par des arrivées de conservateurs d'État.
} 
compétences communes à toutes (par exemple, s'agissant des cadres, la gestion), d'autre part, que la notion de politique culturelle est une donnée qui s'impose et avec elle le partenariat. Le risque est celui d'une sorte de déqualification par le haut (l'amateurisme étant une déqualification par le bas ou plutôt un en deçà de la qualification) : à savoir, la dilution des savoir-faire propres à chaque domaine dans une sorte de "melting-pot" des objectifs et des techniques.

Garant des statuts des personnels, l'État est fondé à appeler l'attention sur ce risque et à y parer, comme à mettre l'accent sur les interrogations et les mises à jour auxquelles il appelle chaque filière.

\subsubsection{Conclusion}

Sous le rapport du rôle que l'État croit devoir être le sien par rapport aux collectivités locales, la situation paraît pouvoir être résumée comme suit.

1) Bon an mal an, l'État, tel que le représente le ministère de la Culture, a identifié des domaines dans lesquels il estime avoir des responsabilités particulières. Il en est ainsi de la coopération et du patrimoine.

Ces responsabilités sont assumées honorablement, et imparfaitement. La coopération, en particulier, reste un immense chantier.

2) Quant aux autres responsabilités - celles qui incombent d'abord aux collectivités territoriales - le problème (pour ne pas dire le dilemme) qui se pose au ministère n'a guère changé par rapport aux années qui ont vu la décentralisation s'engager.

D'un côté, le ministère prend acte qu'il revient aux villes et aux départements de déterminer s'ils veulent disposer d'une bibliothèque moderne et efficace et se doter des moyens nécessaires pour ce faire.

D'un autre côté, d'une part, il estime qu'il est de son devoir d'assurer l'égalité des citoyens, sur tout le territoire, devant l'offre des bibliothèques; d'autre part, il se fait des prestations qu'elles doivent délivrer une haute idée et cherche à les tirer vers le haut.

Du fait de cette double exigence, le ministère croit régulièrement devoir sortir du rôle d'incitateur que lui assigne la décentralisation pour devenir ou redevenir un acteur. Sa récente initiative en faveur de la construction de "médiathèques de proximité" est significative de ce point de vue. Il en est de même de l'aide financière au recrutement de personnel qualifié, mesure récurrente depuis vingt ans, car elle se situe un peu au-delà de la simple incitation, quelque part entre l'incitation et l'aide permanente au fonctionnement, même si elle reste plus proche de l'incitation.

Or, les moyens du ministère sont limités. Structurellement, parce que les moyens sont par définition limités par rapport aux besoins. De surcroît, en ce moment, la France connaît en ce moment des difficultés économiques et budgétaires.

Pour sortir de l'impasse, deux écoles font entendre leur voix. La première - plus représentée à droite qu'à gauche - est celle de "l'État recentré". Présentons sa conception comme suit : si l'État consacrait moins de crédits aux missions dont on peut penser qu'elles ne sont pas ou moins les siennes, il en aurait plus pour remplir ses missions propres.

La seconde école - plus représentée à gauche qu'à droite - est celle de "l’État multiplié". Pour cette école, l'État doit à la fois accomplir au mieux toutes ses missions propres et, en attendant que l'égalité des citoyens devant l'offre en bibliothèques soit assurée sur tout le territoire, intervenir de manière significative pour que cette égalité devienne une réalité.

Il est permis d'espérer que l'école qui l'emportera est la seconde : celle de l'État multiplié. Telle n'est pas la tendance. 
Supposons qu'elle s'inverse. Plus que par le passé, de toute manière, l'État devra s'interroger sur les raisons qui fondent son intervention auprès des collectivités locales, la justifier et donc la rendre justifiable - c'est-à-dire cohérente, conséquente.

De ce point de vue, des évolutions sont plus qu'inéluctables : nécessaires. Les refuser par principe présenterait des risques considérables : quand le rapport de force n'est pas favorable aux partisans du statu quo, ce qui est le cas dans le domaine des bibliothèques, à la radicalité de leur position défensive a toute chance de répondre celle des mesures auxquelles ils entendaient s'opposer.

De ces évolutions, mieux vaut donc accepter la perspective en s'attachant à les infléchir au mieux du développement des bibliothèques. Cette attitude qui est celle de la négociation ne constituerait pas un renoncement; elle s'inscrirait dans le droit fil de la stratégie qui dans les années 1980, devant la décentralisation annoncée, avait conduit la DLL à obtenir - notamment - la création du concours particulier. 


\section{LE FONCTIONNEMENT DE L'INSPECTION GENERALE DES BIBLIOTHEQUES}

L'Inspection générale des bibliothèques est placée sous l'autorité directe du ministre de l'Éducation nationale, de l'Enseignement supérieur et de la Recherche et mise à la disposition du ministre de la Culture et de la Communication.

En 2003, le doyen a présenté les activités de l'Inspection à :

- A. Boissinot, directeur de cabinet du ministre de l'Éducation (28 juillet) ;

- G. Cerruti, directeur de cabinet du ministre de la Culture (6 août).

L'Inspection travaille en étroite collaboration avec la direction des Personnels, de la Modernisation et de l'Administration et la direction du Livre et de la Lecture. Après leur nomination, J.-L. Gautier-Gentès a rencontré :

- D. Antoine, directeur de la DPMA (22 octobre).

- E. Gross, directeur de la DLL (16 juin).

\subsection{LE PERSONNEL}

\subsubsection{Les inspecteurs généraux} généraux.

Au cours de l'année 2003, l'Inspection a fonctionné avec un effectif de huit inspecteurs

T. Bally, partie en retraite le $1^{\text {er }}$ janvier, a été remplacée, à compter du 26 mai, par F. Lemelle, précédemment directrice de la Bibliothèque interuniversitaire d'art et d'archéologie Jacques Doucet. Celle-ci a repris la zone de T. Bally.

D. Renoult, chargé de mission d'inspection générale depuis le $1^{\text {er }}$ janvier 2001 , est mis à disposition du recteur de l'académie de Paris. Il exerce depuis 2003, auprès de celui-ci, les fonctions de chef de la mission interacadémique U3M.

La répartition des emplois est la suivante :

a) emplois du ministère de l'Éducation nationale: un inspecteur général des bibliothèques (D. Pallier), cinq conservateurs généraux des bibliothèques chargés de missions d'inspection générale (J.-L. Gautier-Gentès, F. Lemelle, L. Mérigot, D. Oppetit et D. Renoult) ;

b) emplois du ministère de la Culture : trois conservateurs généraux des bibliothèques chargés de mission d'inspection générale (J.-M. Arnoult, C. Lieber, A. Poirot).

En 2002 et 2003, les distinctions honorifiques suivantes ont été attribuées aux membres de l'Inspection :

- Ordre national de la légion d'honneur : D. Pallier a été nommé chevalier, ainsi que J.-P. Casseyre, inspecteur général honoraire.

- Ordre national du mérite : A. Poirot a été nommé chevalier.

- Ordre des palmes académiques: L. Mérigot a été promue commandeur, et D. Pallier officier; J.-M. Arnoult, D. Oppetit et A. Poirot ont été nommés chevaliers. 


\subsubsection{Le secrétariat}

Le secrétariat ne compte qu'un seul agent. Delphine le Bian, adjoint administratif, assure l'intendance du service, la gestion des agendas des inspecteurs généraux, l'organisation matérielle des missions, la diffusion et l'archivage des rapports, la gestion des statistiques.

Depuis 1997, D. Le Bian a constitué et alimente un site "Inspection générale des bibliothèques" sur le réseau Internet du ministère de l'Éducation nationale. Ce site, régulièrement mis à jour, indique les coordonnées du service et de ses membres, les présidences de jurys. Il donne accès aux publications récentes de l'Inspection : rapports annuels, rapports de concours et d'examens professionnels, auxquels les bibliothèques sont incitées à recourir.

Les inspecteurs généraux sont fréquemment en déplacement. Il est cependant toujours possible de les joindre, par téléphone (sur répondeur en cas d'absence de la secrétaire), par fax ou par messagerie électronique (voir annexe 8, leurs adresses personnelles).

\subsection{ORGANISATION MATERIELLE}

\subsubsection{Locaux}

Les travaux confiés aux inspecteurs généraux (contrôle, études, organisation et gestion de jurys de recrutement, participation aux commissions paritaires et à de nombreux groupes de travail) supposent une présence fréquente, la disposition de bureaux, d'ordinateurs et de moyens de communication.

L'Inspection fonctionne désormais sur 3 sites :

- Locaux du ministère de l'Éducation nationale : le siège de l'Inspection (direction, secrétariat) se trouve dans des locaux du ministère de l'Éducation nationale ainsi que les bureaux de cinq des six inspecteurs ressortissant au ministère de l'Éducation nationale. Depuis janvier 2004, l'Inspection est installée au 10 rue Auber, Paris $9^{\text {ème }}$. Elle y dispose de six bureaux, deux salles de documentation, dont l'une permet, sinon d'offrir un lieu de travail permanent aux inspecteurs relevant du ministère de la Culture, du moins de les accueillir lors de leurs passages, et d'une salle d'archives. Les coordonnées téléphoniques et les adresses électroniques restent inchangées.

- Locaux de la direction du Livre et de la Lecture : depuis mars 2002, les trois inspecteurs mis à disposition par le ministère de la Culture et de la Communication, disposent, dans les locaux de la DLL au 180 rue de Rivoli, Paris $1^{\text {er }}$, de deux pièces dont l'une partagée avec le chargé de mission pour les études de la DLL.

- DRAC de Bourgogne : l'appartenance d'A. Poirot à l'Inspection générale des bibliothèques s'est accompagnée d'une résidence administrative en région et, à partir de l'année 2000, d'une installation dans des bureaux dépendant de la DRAC de Bourgogne à Dijon.

\subsubsection{Crédits et équipement}

Depuis 1996, l'Inspection fonctionne avec des moyens fournis par deux ministères.

Le ministère de l'Éducation nationale fournit les moyens de fonctionnement de cinq inspecteurs et du secrétariat. Le ministère de la Culture et de la Communication prend en charge les moyens de fonctionnement des trois inspecteurs qu'il met à disposition. Quelle que soit l'origine des moyens, chacun des huit inspecteurs assure le suivi des bibliothèques universitaires et des bibliothèques territoriales dans les zones géographiques qui lui sont attribuées. 


\subsubsection{Le ministère de la Culture}

Le ministère de la Culture et de la Communication (direction du Livre et de la Lecture), prend en charge les frais de missions, le matériel informatique, les matériels et les frais de télécommunications de J.-M. Arnoult, C. Lieber et A. Poirot. En 2003, les frais de missions payés par la direction du Livre et de la Lecture au titre de l'Inspection se sont élevés à 10250 euros.

\subsubsection{Le ministère de l'Éducation nationale}

Au ministère de l'Éducation nationale, l'origine des moyens de fonctionnement de l'IGB a été fixée en 1996 par accord avec la direction chargée du fonctionnement de l'administration centrale et la direction chargée des bibliothèques. Depuis, les membres de l'Inspection sur emplois du ministère ont reçu leurs moyens de fonctionnement de la direction de l'Administration (DA) et de la direction de l'Enseignement supérieur (DES), en charge des bibliothèques.

En 2001, une évolution s'est produite vers l'individualisation des moyens propres du service.

Au cours de cette année, le matériel informatique avait été renouvelé et complété. En 2003, le parc des postes informatiques fixes a été partiellement renouvelé.

Le bureau des missions et des déplacements (DPMA C8) gère les frais de déplacement. En 2003, l'enveloppe de l'Inspection a été de 13000 euros pour les missions en métropole et 4389 euros pour les missions hors métropole (IFLA à Berlin, LIBER à Rome, mission d'inspection du SCD de Polynésie française et mission d'étude sur l'état de la lecture publique dans le territoire).

\subsection{LE FONCTIONNEMENT}

\subsubsection{La réforme du statut}

Pendant une longue période, l'IGB avait bénéficié d'un cadre de fonctionnement simple. Elle constituait un corps. De 1945 à 1975, elle a été l'auxiliaire d'une direction chargée des bibliothèques publiques et universitaires et de leurs personnels d'État, qui organisait l'ensemble des tâches de l'Inspection (contrôle, études, jurys...).

Diverses évolutions sont intervenues depuis :

En 1975, les attributions du ministre de l'Éducation nationale en matière de bibliothèques publiques ont été transférées au ministre de la Culture. L'Inspection, dont l'ensemble des moyens demeurait au ministère de l'Éducation nationale, a été mise à la disposition du ministre de la Culture pour les bibliothèques qui relevaient de sa compétence.

En 1989, la gestion des personnels de bibliothèques a été rattachée à une direction spécialisée du ministère de l'Éducation nationale.

A partir de 1990, pour une meilleure organisation du fonctionnement de l'Inspection, qui travaillait désormais pour trois directions, l'IGB a reçu des programmes ministériels.

$>$ En 1992 est intervenue la mise en extinction du corps des inspecteurs généraux des bibliothèques. Le décret du 9 janvier 1992 portant nouveau statut du corps des conservateurs des bibliothèques et statut du corps des conservateurs généraux des bibliothèques prévoyait que des missions d'inspection générale seraient confiées à des conservateurs généraux ou à des conservateurs en chef des bibliothèques.

Pour garantir aux bibliothèques un contrôle continu et homogène, la solution la plus simple était de constituer un service, composé des derniers inspecteurs généraux et de conservateurs généraux chargés de missions d'inspection générale. C'est la décision qui a été prise et appliquée immédiatement pour le fonctionnement des Inspections des archives, des musées et du patrimoine, secteurs où les statuts des conservateurs avaient évolué de manière analogue. Mais, dans le cas de 
l'Inspection générale des bibliothèques, fonctionnant dans un cadre interministériel, deux questions devaient être réglées :

- la définition d'un service d'inspection générale des bibliothèques composé pour l'essentiel de chargés de missions d'inspection générale, prenant le relais du corps des inspecteurs généraux des bibliothèques,

- le mode de fonctionnement interministériel du service d'inspection (définition des programmes, diffusion des rapports, nomination des membres du service et du doyen, rôle de ce dernier).

Le principe d'une organisation permanente de l'Inspection, la décision de constituer un service d'inspection générale des bibliothèques ont fait l'objet de deux arbitrages ministériels.

Le premier arbitrage a été rendu en juillet 1992 par le cabinet de M. J. Lang, ministre de l'Éducation nationale et de la Culture. L'arrêté fixant l'organisation du service d'inspection a été élaboré en 1994 par la direction de l'Information scientifique et Technique et des Bibliothèques (DISTB), en liaison avec la direction du Livre et de la Lecture (DLL). Ce texte a été validé par le cabinet du ministre de l'Enseignement supérieur et de la Recherche. En 1995-1996, l'effectif de l'Inspection a été reconstitué grâce à un apport de postes de la DISTB et de la DLL. L'IGB a recommencé à fonctionner dans un cadre de fait.

Après avoir fait l'objet de demandes de corrections du Secrétariat général du Gouvernement, puis de la direction des Affaires juridiques du ministère de l'Éducation nationale, le texte a été soumis à un nouvel arbitrage en 1998. M. Garden, consultant permanent du ministre de l'Éducation nationale, a souligné la nécessité d'un service d'inspection générale des bibliothèques, constitué de conservateurs généraux dont l'effectif pourrait être supérieur au nombre actuel, et a conclu à la relance du processus de création du service. M. C. Allègre, ministre de l'Éducation nationale, de la Recherche et de la Technologie, a donné son accord à ces conclusions. Parallèlement, au cours de la préparation du projet de loi sur les bibliothèques, le ministère de la Culture et de la Communication avait fait part de son vœu de renforcer et d'élargir le rôle de l'Inspection.

En 1999, la direction des Affaires juridiques (DAJ) a fait connaître son avis sur le projet de décret relatif aux missions et à l'organisation de l'Inspection générale des bibliothèques. Ce dossier a été examiné dans le cadre d'une réunion entre directions (direction de l'Enseignement supérieur direction des Affaires juridiques) présidée par M. J.-R. Cytermann, directeur adjoint du cabinet du ministre, le 17 mai.

Il a alors été décidé :

1) d'ajouter systématiquement à l'arrêté d'organisation du ministère de l'Éducation nationale qu'il existe trois inspections générales: l'Inspection générale de l'Éducation nationale (IGEN), l'Inspection générale de l'administration de l'Éducation nationale et de la Recherche (IGAENR), l'Inspection générale des bibliothèques (IGB) ;

2) de modifier le décret régissant les conservateurs et conservateurs généraux, de telle façon que le ministre de la Culture puisse être consulté sur la nomination des conservateurs généraux chargés de mission d'inspection et qu'un doyen puisse être désigné après avis du même ministre.

Une circulaire interministérielle devait par ailleurs mettre en forme les procédures de fonctionnement (programme, répartition des tâches, missions d'intérêt commun, missions hors cadre des ministères de l'Éducation nationale et de la Culture, diffusion des rapports, rapport annuel).

La seconde décision a été matérialisée par la parution, au Journal officiel du 18 octobre 2001, du décret $n^{\circ}$ 2001-946 du 11 octobre modifiant le décret $n^{\circ}$ 92-26 du 9 janvier 1992 portant statut particulier du corps des conservateurs de bibliothèques et du corps des conservateurs 
généraux des bibliothèques. Entre autres dispositions, ce texte, d'une part, officialise le droit du ministre de la Culture à émettre un avis sur la nomination de tous les conservateurs généraux chargés de mission d'inspection générale, d'autre part, fait du décanat, fonction de fait exercée par un doyen désigné, une fonction officielle confiée à un doyen nommé. En effet, d'après ce texte, un doyen des conservateurs et conservateurs généraux chargés de mission d'inspection générale, nommé après avis du ministre de la Culture, "dirige, anime et coordonne leurs activités" et "centralise les conclusions de leurs travaux".

J.-L. Gautier-Gentès avait été désigné comme doyen de l'Inspection générale à compter du $1^{\text {er }}$ janvier 2000. En application de ce décret, il a été nommé en cette qualité, par arrêté du ministre de l'Éducation nationale en date du 3 juillet 2002, pris après avis de la ministre de la Culture.

S'il ne s'agit pas encore du véritable cadre attendu pour que l'Inspection puisse accomplir ses missions - à commencer par le contrôle et l'évaluation - dans les conditions appropriées, ces dispositions constituent une première avancée significative.

\subsubsection{Organisation administrative}

Le doyen - dont il est rappelé qu'il cumule cette charge avec la responsabilité d'une zone d'inspection entière - organise la préparation des programmes, assure la coordination des contacts avec les administrations, et réunit périodiquement les inspecteurs pour des séances de travail et d'information (21 janvier, 2 mars, 29 avril, 11 juin, 15 juillet, 3 septembre, 9 octobre, 27 novembre). Il assume la responsabilité du rapport annuel, dont la rédaction a été assurée par $D$. Pallier et F. Lemelle.

Chacun des inspecteurs est responsable du suivi d'une zone géographique, dans laquelle il assure les inspections prévues au programme annuel et les missions demandées en cours d'année. Ces zones ont été redistribuées en 2001, sans modification de leur contenu. Il est prévu que les inspecteurs conservent leurs zones respectives 5 ans en moyenne. On trouvera en annexe 6 la répartition actuelle.

Chaque inspecteur participe à la CAP d'au moins une catégorie de personnels et assure, le cas échéant, la responsabilité d'un jury concernant cette catégorie.

Les trois conservateurs généraux sur emplois du ministère de la Culture et de la Communication sont régulièrement appelés à participer aux réunions des bureaux de la DLL.

J.-M. Arnoult est membre du comité technique paritaire de la direction du Livre et de la Lecture. C. Lieber est suppléante.

\subsubsection{Contrôle}

Lors de la préparation de chaque mission, l'inspecteur concerné prend contact avec la tutelle de l'organisme documentaire à inspecter et avec le responsable de cet organisme. L'inspection est confirmée par écrit, avec copie pour information aux administrations d'État concernées.

En ce qui concerne les bibliothèques publiques, les inspections sont préparées avec les directions régionales des Affaires culturelles. Une collaboration étroite entre les DRAC et l'Inspection générale, axée principalement sur des échanges réguliers d'informations, conditionne l'efficacité du contrôle que l'État souhaite exercer localement, et du dialogue qu'il entretient avec les collectivités locales. Les échanges avec les rectorats sont traditionnellement moins structurés que ceux existant dans le domaine culturel, alors que les moyens des bibliothèques de l'enseignement supérieur restent assez dépendants des administrations centrales.

Chaque inspection fait l'objet d'un rapport. Pour leur élaboration, les inspecteurs utilisent des cadres communs, validés avec les directions chargées des bibliothèques. 
Suivant le Code général des collectivités territoriales, chaque inspection donne lieu à un rapport au ministre chargé de la Culture, qui est ensuite transmis par le préfet de région au maire ou au président de conseil général. Au ministère de l'Éducation nationale, depuis 1998, les rapports sont communiqués au ministre et à ses collaborateurs mais également aux recteurs et aux présidents ou directeurs des établissements concernés.

Il est rappelé que l'IGB n'est pas habilitée à diffuser elle-même ses rapports auprès des collectivités. Au sein de celles-ci, la communication des rapports aux directeurs des bibliothèques inspectées est du ressort des responsables des collectivités.

Il appartient au ministre de la Culture - c'est-à-dire, en l'occurrence, au directeur du Livre et de la Lecture - d'adresser les rapports de l'IGB aux collectivités territoriales concernées. De ce point de vue, ont été observés au cours des dernières années des retards nombreux et importants. Il a été proposé par le nouveau directeur du Livre que la remise de chaque rapport soit immédiatement suivie d'un entretien avec lui, au cours duquel seraient en particulier arrêtés les termes du courrier d'envoi. Cette procédure a commencé d'être appliquée. Elle constitue une nette amélioration. Il y aura lieu d'en évaluer les effets en 2004. 


\section{ANNEXES}

Annexe $1 \quad$ Établissements inspectés ou visités en 2003

Annexe 2 Travaux et publications de l'IGB en 2003

Annexe $3 \quad$ Revue de presse

Annexe $4 \quad$ Jurys de concours présidés par les IGB en 2003 Synthèse des rapports

Annexe $5 \quad$ Textes concernant l'IGB

État au 31 décembre 2003

Annexe 6

Répartition des zones d'inspection (2004)

Annexe 7

Présidences de jurys de concours et d'examen (2004)

Annexe 8

Informations pratiques concernant l'IGB 


\section{ÉTABLISSEMENTS INSPECTES OU VISITES EN 2003}

\begin{tabular}{|c|c|c|c|}
\hline RÉGIONS & IG & CONTRÔLES & VISITES \\
\hline AQUITAINE & J.-L. Gautier-Gentès & BM de Mont-de-Marsan & $\begin{array}{l}\text { BM de Billère } \\
\text { BMC de Bordeaux } \\
\text { BMC de Pau }\end{array}$ \\
\hline BOURGOGNE & J.-M. Arnoult & & BMC Dijon \\
\hline BRETAGNE & L. Mérigot & $\begin{array}{l}\text { SCD Rennes } 1 \\
\text { BDP Ille-et-Vilaine }\end{array}$ & BMC Rennes \\
\hline CENTRE & A. Poirot & BMC Bourges & $\begin{array}{l}\text { BM Chartres } \\
\text { BM Châteauroux } \\
\text { BM Lucé }\end{array}$ \\
\hline CHAMPAGNE-ARDENNE & A. Poirot & $\begin{array}{l}\text { SCD Reims-Champagne- } \\
\text { Ardenne }\end{array}$ & $\begin{array}{l}\text { BMC Reims } \\
\text { BM Saint-Brice-Courcelles }\end{array}$ \\
\hline FRANCHE-COMTÉ & A. Poirot & SCD Franche-Comté & \\
\hline ÎLE-DE-FRANCE & & $\begin{array}{l}\text { BIUS Jussieu (DP) } \\
\text { SCD Paris } 7 \text { (DP) }\end{array}$ & $\begin{array}{l}\text { BDIC (LM) } \\
\text { SCD Paris } 13(\mathrm{DO}) \\
\text { BM Fontainebleau (CL) } \\
\text { BM Mantes-la-Jolie (JMA) }\end{array}$ \\
\hline LANGUEDOC-ROUSSILLON & C. Lieber & BM Sète & \\
\hline LORRAINE & J.-M. Arnoult & & $\begin{array}{l}\text { BM Bar-le-Duc } \\
\text { BM Épinal } \\
\text { B. Saint-Mihiel }\end{array}$ \\
\hline BASSE-NORMANDIE & L. Mérigot & $\begin{array}{l}\text { Caen-la-Mer (BMC Caen } \\
\text { et BM Hérouville) }\end{array}$ & \\
\hline PACIFIQUE & J.-L. Gautier-Gentès & $\begin{array}{l}\text { SCD de l'Université de } \\
\text { Polynésie française }\end{array}$ & $\begin{array}{l}\text { Bibliothèque de la maison } \\
\text { de la culture de Papeete }\end{array}$ \\
\hline PAYS-DE-LA-LOIRE & F. Lemelle & $\begin{array}{l}\text { SCD Angers } \\
\text { BMC du Mans }\end{array}$ & \\
\hline POITOU-CHARENTES & F. Lemelle & $\begin{array}{l}\text { SCD La Rochelle } \\
\text { BMC La Rochelle }\end{array}$ & \\
\hline PROVENCE-ALPES-CÔTE D’AZUR & C. Lieber & $\begin{array}{l}\text { SCD Aix-Marseille } 1 \\
\text { BM Briançon } \\
\text { BM Sanary-sur-Mer } \\
\text { Médiathèque intercommunale } \\
\text { du SAN Ouest-Provence }\end{array}$ & $\begin{array}{l}\text { BMC Aix-en-Provence (JMA) } \\
\text { BM Martigues }\end{array}$ \\
\hline RHÔNE-ALPES & D. Pallier & & $\begin{array}{l}\text { SICD Grenoble } 2 \\
\text { Médiathèque de la Loire } \\
\text { BM de Montbrison } \\
\text { B. Collégiale de Saint-Bonnet- } \\
\text { le-Château }\end{array}$ \\
\hline & & 20 contrôles & 24 visites \\
\hline AUTRES (7) & & \multicolumn{2}{|c|}{$\begin{array}{l}\text { Audit de la } \mathrm{BnF}(\mathrm{DO}) \\
\text { Bibliothèque marxiste de Paris (DO) }\end{array}$} \\
\hline & & \multicolumn{2}{|c|}{ ENSAM, bibliothèques d'Angers et de Châlons-en-Champagne (JMA) } \\
\hline & & \multicolumn{2}{|c|}{ Mission d'expertise du CIRDOC (CL) } \\
\hline & & \multicolumn{2}{|c|}{ Mise en réseau des SCD de Haute-Normandie (LM) } \\
\hline & & \multicolumn{2}{|c|}{ ENSSIB (DP) } \\
\hline ÉTRANGER (2) & & \multicolumn{2}{|l|}{$\begin{array}{l}\text { Etat du patrimoine irakien (JMA) } \\
\text { Bibliothèques afghanes (JMA) }\end{array}$} \\
\hline & & TOTAL & 53 missions \\
\hline
\end{tabular}


ANNEXE 2

\section{TRAVAUX ET PUBLICATIONS}

DE L'INSPECTION GENERALE DES BIBLIOTHEQUES EN 2003

\section{RAPPORTS}

\section{A - Rapports d'inspections et de visites}

36

\section{B - Rapports thématiques}

D. Oppetit : Rapport d'audit de la BnF, en vue de la préparation d'un contrat d'objectifs et de moyens, présenté par R. Klein, inspecteur général de l'administration des affaires culturelles et D. Oppetit, inspectrice générale des bibliothèques, 4 tomes + annexes (rapport remis au directeur de cabinet du ministre de la Culture et de la Communication).

\section{C - Rapports de jurys de concours}

- J-M. Arnoult : Rapport sur le concours de recrutement de magasiniers en chef, session 2003, septembre 2003.

- F. Lemelle : Rapport sur l'examen professionnel pour l'accès au grade d'assistant des bibliothèques de classe exceptionnelle, session 2003, novembre 2003.

- L. Mérigot :

- Rapport sur le concours de recrutement de bibliothécaires adjoints spécialisés, session 2003, octobre 2003.

- Rapport sur l'examen professionnel pour l'accès au grade d'assistant des bibliothèques de classe exceptionnelle, session 2002, mars 2003.

- D. Pallier :

- Concours de recrutement de bibliothécaires. Fonction publique d'État. Concours externeconcours interne. Annales. Session 2002. ENSSIB, Villeurbanne, 2003, 148 p.

- Rapport sur le concours de recrutement de conservateurs stagiaires élèves de l'ENSSIB, réservé aux élèves et anciens élèves de l'École nationale des chartes, session 2002, février 2003.

- A. Poirot : Concours réservé pour le recrutement de bibliothécaires d'État, $1^{\text {ère }}$ session (octobre-décembre 2002), rapport du jury par A. Poirot, juillet 2003. 


\section{D - Rapport annuel}

- Rapport d'activité de l'Inspection générale des bibliothèques, 2002, par D. Oppetit, avec la collaboration de D. Pallier, juin 2003.

\section{PUBLICATIONS SCIENTIFIQUES ET PROFESSIONNELLES}

\section{$>$ J.-L. GAUTIER-GENTÈS}

- "Évaluation du diplôme de conservateur", Bulletin des bibliothèques de France, 2003, t. $48, \mathrm{n}^{\circ} 1, \mathrm{p} \cdot 16-27$.

- "Refonder les bibliothèques municipales: préliminaires", Bulletin des bibliothèques de France, 2003 , t. $48, \mathrm{n}^{\circ} 2$, p. $67-80$.

- "Bibliothèques publiques et laïcité : de la neutralité au pluralisme ?", BIBLIOthèque(s). Revue de l'Association des bibliothécaires français, $\mathrm{n}^{\circ}$ 11-12, décembre 2003, p. 68-86.

\section{PALLIER}

- "Évaluation de la formation initiale des bibliothécaires", dans Bulletin des bibliothèques de France, 2003, $\mathrm{n}^{\circ} 1$, pp. 28-34. 
ANNEXE 3

\section{REVUE DE PRESSE 2003}

A l'occasion de la publication du rapport annuel 2002 de l'Inspection générale des bibliothèques, un entretien avec le doyen a été publié sous le titre "Il ne suffit pas d'inspecter" dans Livre hebdo, $\mathrm{n}^{\circ} 524,5$ septembre 2003, p.75.

Un compte rendu du rapport annuel 2002 est paru dans le Bulletin des bibliothèques de France, 2004, $\mathrm{n}^{\circ} 1$, p. 113-115.

Un résumé de la mission de J.-M. Arnoult en Irak a été publié sous le titre "Bagdad six mois après" dans Livre hebdo, n531, 24 octobre 2003, p. 64-65.

Un autre article de J.-M. Arnoult "Les bibliothèques irakiennes en 2003 : un nouveau chapitre de l'histoire interminable des désastres" a été publié dans International preservation news, 30 (2003), p. 20-29. 
ANNEXE 4

\section{CONCOURS PRESIDES PAR LES INSPECTEURS GENERAUX EN 2003}

\section{SYNTHESE DES RAPPORTS}

\section{Concours d'entrée à l'ENSSIB réservé aux élèves et anciens élèves de l'École nationale des chartes, session 2003.}

En 2003, ce concours a été ouvert plus tôt (arrêté du 29 avril 2003), suivant la proposition de l'École des chartes et après concertation entre la DPMA, la DES (SDBD) et l'IGB. Ainsi, pour les chartistes, les calendriers des inscriptions au concours d'entrée à l'ENSSIB et au concours d'entrée à l'Institut national du Patrimoine sont désormais alignés. Comme les années précédentes, le nombre de postes proposés au "concours chartiste" s'élevait à 15 (arrêté du 18 juin 2003).

20 candidats s'étaient inscrits. 18 se sont présentés. Ils représentaient inégalement les deux filières de l'école : 14 appartenaient à la filière $A$ (médiévale et moderne) et 4 à la filière $B$ (moderne et contemporaine). 13 étaient des élèves de troisième année, admis à soutenir leur thèse d'École des chartes. 5 étaient déjà archivistes paléographes. A nouveau, tous les candidats sauf un avaient obtenu un DEA.

Le jury, présidé par D. Pallier, comptait cinq membres. La première épreuve (examen des dossiers) a eu lieu à l'Inspection générale des bibliothèques. Suivant l'organisation fixée depuis 1997, la seconde épreuve (entretien avec les candidats), s'est déroulée dans les locaux de la Bibliothèque nationale de France.

Pour la liste principale de 15 noms, la barre d'admission a été en 2003 de 11,67/20. La candidate classée première a obtenu une moyenne de 16,26/20. Une liste complémentaire de deux noms a été dressée. Constatant la motivation et la préparation très insuffisantes d'un candidat, le jury ne l'a pas classé.

Comme par le passé, plusieurs élèves se présentaient à la fois au concours de l'ENSSIB et au concours de l'Institut national du patrimoine (spécialités archives et musées). A la suite du processus de désistement de candidats, 12 chartistes ont commencé leur scolarité à l'ENSSIB en janvier 2004.

\section{Concours de bibliothécaires d'État réservé, session 2002}

Ce concours a été ouvert par l'arrêté du 7 juin 2002 ; un contingent de 12 emplois lui a été fixé par l'arrêté du 16 septembre. Pour son organisation, il a associé l'ENSSIB et le bureau des concours (DPMA B7) du ministère de l'Éducation nationale.

Ce concours, d'un type entièrement nouveau, faisait suite à la loi $\mathrm{n}^{\circ}$ 2001-2 du 3 janvier 2001 relative à la résorption de l'emploi précaire et à la modernisation du recrutement dans la fonction publique ainsi qu'au temps de travail dans la fonction publique territoriale. Cette loi, dite Loi Sapin, a donné lieu aux textes d'application suivants :

- Le décret $n^{\circ}$ 2001-834 du 12 septembre 2001 relatif à la reconnaissance de l'expérience professionnelle en équivalence des conditions de titres ou de diplômes requises pour se présenter aux concours et examens professionnels réservés organisés en application de l'article $1^{\text {er }}$ de la loi $n^{\circ}$ 2001-2 du 3 janvier 2001 (...). 
- Le décret $n^{\circ}$ 2002-427 du 27 mars 2002 portant organisation de concours de recrutement de fonctionnaires de l'État des catégories A, B et C (...), en application de l'article $1^{\text {er }}$ de la loi $n^{\circ}$ 2001-2 du 3 janvier 2001 (...).

- L'arrêté du 27 mars 2002 fixant les règles d'organisation générale, la nature et le programme des épreuves des concours réservés institués par le décret $n^{\circ}$ 2002-427 du 27 mars 2002 pour l'accès au corps des bibliothécaires.

- L'arrêté du 27 mars 2002 fixant les règles de fonctionnement et de constitution des commissions instituées par le décret $n^{\circ}$ 2001-834 relatif à la reconnaissance de l'expérience professionnelle (...).

- La circulaire $n^{\circ}$ 2002-121 du 29 mai 2002, émanant de la DPATE, intitulée Résorption de l'emploi précaire : organisation des concours et examens professionnels réservés d'accès aux corps IATOSS.

Des textes plus anciens permettaient également de cadrer l'organisation du concours : bibliothécaires.

- Le décret $\mathrm{n}^{\circ}$ 92-29 du 9 janvier 1992 portant statut particulier du corps des

- L'arrêté du 12 février 1992 fixant les modalités d'organisation des concours de recrutement des bibliothécaires.

- L'arrêté du 12 février 1992 fixant la liste des titres et diplômes exigés des candidats au concours externe de recrutement de bibliothécaires.

Pour sa session 2002, de nombreuses incertitudes ont jalonné l'organisation du concours. Le premier calendrier étalé sur le premier semestre a été annulé faute de pouvoir bénéficier des textes réglementaires nécessaires selon des délais raisonnables. Ces incertitudes ont également porté sur le nombre des candidats éventuels pouvant répondre aux critères décidés par le législateur ; la recevabilité des dossiers déposés par les "sapinisables" - selon le jargon spontané engendré par le dispositif - méritait donc un examen très rigoureux. De plus, une commission a été instituée pour la reconnaissance des expériences professionnelles en équivalence des conditions de titres et de diplômes ; présidée par Mme Catherine Gaillard, chargée de mission à la SDBD, elle a statué en amont de l'épreuve écrite. Cette commission et le jury du concours ont fonctionné de façon distincte.

La première épreuve s'est déroulée le 8 octobre dans deux centres (Paris et La Réunion). Les entretiens ont eu lieu à l'ENSSIB, les 16 et 17 décembre. Les résultats étaient disponibles sur le site de l'ENSSIB dès le jeudi 19.

Les 47 candidats travaillaient dans des établissements dépendant des ministères de la Culture et de la Communication et de la Jeunesse, de l'Éducation nationale et de la Recherche. Trois ne se sont pas présentés à l'épreuve écrite ; conformément aux textes, celle-ci consistait en la rédaction d'une note à partir d'un dossier technique composé d'articles pour l'essentiel tirés de revues professionnelles ; ce dossier était centré sur la formation des utilisateurs ; la note ne devait pas être une note de synthèse, mais répondre à la demande d'un chef de service pour les besoins concrets d'un établissement.

La barre d'admissibilité s'est établie à 8 ; 21 candidats ont été appelés à passer la seconde épreuve qui était composée d'un entretien d'une demi-heure : 10 minutes pour la présentation de son expérience professionnelle par le candidat, 20 minutes d'échanges portant sur cette expérience et plus largement sur ses connaissances en matière de bibliothèques et de documentation. Sur ce dernier point, le jury a été unanime pour constater la grave absence de culture professionnelle des personnes qu'il a entendues ; celles-ci peuvent naturellement être très expertes dans leur domaine (langue rare, support, connaissances informatiques...), mais ne rien savoir de l'environnement général des bibliothèques ; certaines réponses ont même laissé pantois des membres du jury par ailleurs bien disposés vis-à-vis de contractuels qui pour certains sont en poste depuis une quinzaine d'années. 
Ce regret conduit à plusieurs types d'interrogations. L'objectif de ce type de concours est de titulariser des personnes qui, par suite, ont vocation à muter d'un établissement à l'autre ; entrent ainsi dans le corps des bibliothécaires des agents qui sont très loin d'avoir toutes les connaissances de base requises et qui auront de ce fait des difficultés à s'adapter à de nouveaux postes. On s'étonnera ensuite que des établissements, depuis longtemps employeurs, n'aient pas été en mesure de fournir une formation élémentaire de base permettant à ces agents du service public de situer leurs tâches dans un ensemble. Des épreuves écrites d'une autre nature auraient sans doute permis de mieux tester les connaissances professionnelles, quoique les lacunes soient tellement partagées entre les candidats que les résultats n'auraient pas nécessairement été très différents ; en effet, la première épreuve révélait tout de même la capacité des meilleurs d'entre eux à faire face à une question qui n'entrait pas forcément dans leurs préoccupations quotidiennes immédiates. Enfin, on aurait aimé que, dans cette situation, les lauréats du concours aient pu bénéficier à la suite de leur succès d'un cycle de formation de base; espérons que les établissements affectataires, dans leur intérêt propre, auront à cœur de permettre à leurs agents de combler leurs lacunes les plus lourdes.

La lauréate classée première a obtenu une moyenne de 14,8. Le jury n'a pas dressé de liste complémentaire. Les 12 lauréats - 9 de l'Éducation nationale, 3 de la Culture - ont été nommés stagiaires le $1^{\text {er }}$ février 2003 ; l'administration a examiné chaque dossier avec la plus grande attention pour que leur affectation réponde au mieux à leurs aspirations dans les cas où aucun poste vacant ne permettait une nomination sur place. Pour deux lauréats, la réussite à ce concours s'est traduite par leur installation dans une autre ville que celle où ils résidaient jusque là.

\section{Concours de recrutement de bibliothécaires adjoints spécialisés}

Par dérogation aux dispositions prévues dans les articles 4 et 6 du décret du 9 janvier 1992, le concours interne était ouvert "au titre de l'année 2003 aux assistants de bibliothèques justifiant de quatre ans de services effectifs dans le corps." C'était la dernière année des modalités exceptionnelles de recrutement dans le corps des bibliothécaires adjoints spécialisés. Le concours interne comportait de ce fait une épreuve unique notée de 0 à 20 , consistant en un entretien avec le jury d'une durée de 30 minutes.

L. Mérigot présidait le jury qui, avec 52 membres, hors épreuves de langues, avait été notablement augmenté, essentiellement pour le concours interne en raison du grand nombre de candidats inscrits.

Si le calendrier était sensiblement le même que pour le concours 2002, le nombre d'emplois offerts était en forte augmentation : 95 postes étaient proposés au concours externe dont 87 pour le MJENR et 8 pour le ministère de la Culture, et 126 postes au concours interne dont 116 pour le MJENR et 10 pour le ministère de la Culture.

Le nombre d'inscriptions a été en nette augmentation par rapport à l'année 2002, particulièrement pour le concours interne où 171 candidats étaient inscrits, et également pour le concours externe ; néanmoins sur les 556 candidats inscrits, 479 seulement ont été jugés recevables : de fait, cette année encore, les candidats non admis à présenter le concours ne possédaient pas les diplômes requis (DUT ou DEUST).

351 candidats se sont présentés à l'épreuve de rédaction de notices et 349 à l'épreuve de composition. Le sujet de cette épreuve portait sur les périodiques aujourd'hui dans les bibliothèques. Les résultats ont été très moyens par rapport à ce qu'un sujet large et d'actualité pouvait laisser espérer, la moyenne des notes se situant à 9,3 et $44 \%$ des copies seulement ayant une note égale ou supérieure à la moyenne. Les résultats ont été encore plus médiocres pour la rédaction de notices puisque seuls $30 \%$ des candidats ont une note égale ou supérieure à la moyenne, que 87 copies ont reçu une note éliminatoire et que la moyenne des notes est de 7,62.

Le jury constate que bon nombre de candidats ne semblent ni bien connaître ni avoir manipulé suffisamment les normes ; ils analysent mal les documents, ne connaissent pas la ponctuation prescrite ni avec précision le système d'abréviations. 
Ils ne sont pas sensibles non plus à la question des accès, primordiale pour l'utilisation d'un catalogue. Le jury regrette que les candidats ne soient pas suffisamment préparés à cette épreuve ; ce constat est fait chaque année.

190 candidats ont été déclarés admissibles, le seuil d'admissibilité ayant été fixé à $41 / 100$, soit une moyenne de 8,46 .

Les épreuves d'admission ont été compliquées par la grève des transports parisiens et de la SNCF prévue et prise en compte pour le 13 mai mais pas pour le lendemain, ce qui a nécessité de la part des membres du jury et des candidats beaucoup de souplesse et de bonne volonté.

159 candidats se sont présentés aux épreuves orales et si, pour l'épreuve de conversation, la moyenne des notes $(11,18)$ est comparable aux années précédentes, elle est plus basse pour la recherche documentaire $(9,66)$ où la préparation des candidats est nettement insuffisante et trop théorique. D'une manière générale on constate un manque de connaissances professionnelles de base et même citoyennes.

159 candidats sur les 166 inscrits, se sont présentés au concours interne qui était ouvert cette année à des agents ayant moins d'ancienneté, donc plus jeunes. Le jury a apprécié la qualité des candidats, qui pour la plupart s'étaient préparés à cette épreuve qui ne peut être improvisée. La grande majorité des candidats ont su structurer l'exposé et présenter de manière réfléchie et concrète leur parcours professionnel.

126 candidats ont été déclarés admis, 29 inscrits sur la liste complémentaire, la moyenne générale de l'épreuve étant de 14,63/20.

\section{Concours magasiniers en chef}

Par arrêté du 23 octobre 2002, a été autorisée au titre de l'année 2003 l'ouverture d'un concours pour le recrutement de magasiniers en chef.

Le nombre total d'emplois offerts aux concours était fixé à 66 par arrêté du 20 janvier 2003 (13 pour le concours externe, 53 pour le concours interne).

Le jury était présidé par J.-M. Arnoult.

Les épreuves d'admissibilité se sont déroulées le 28 janvier 2003, à Paris et dans divers centres de province et d'Outre-mer. Les épreuves orales (admission) ont eu lieu du 22 au 24 avril 2003. La réunion d'admission a eu lieu le 25 avril.

L'arrêté du 6 septembre 1995 fixant les modalités d'organisation des concours de recrutement de magasiniers en chef, prévoit pour le concours externe et le concours interne, deux épreuves écrites d'admissibilité :

$1^{\circ}$ ) rédaction, à partir de données communiquées aux candidats, d'une note sur la résolution d'un problème pratique relatif à une situation à laquelle un magasinier en chef peut être confronté (durée : deux heures ; coefficient 3) ;

$2^{\circ}$ ) questionnaire portant sur l'organisation et le fonctionnement des bibliothèques, l'informatique appliquée aux bibliothèques, la gestion, la communication et la conservation des collections (durée : une heure ; coefficient 2).

Une note inférieure à 5 à l'une ou l'autre de ces épreuves est éliminatoire.

Les mêmes épreuves sont données au concours externe et au concours interne. Les candidats au concours interne ne sont pas défavorisés puisqu'ils sont notés et classés à part.

A l'issue des épreuves écrites, 91 candidats externes et 124 candidats internes ont été déclarés admissibles. 


\section{1) Épreuves écrites}

- A l'épreuve de rédaction d'une note, on constate que beaucoup de candidats manquent d'esprit pratique et oublient les aspects les plus simples et les plus élémentaires d'une opération qui n'avait rien d'inhabituel ni de compliqué dans son élaboration ni dans son déroulement. Quant à la forme même de la note, outre que les candidats sont souvent mal à l'aise pour la rédiger, ils ne prennent pas le temps de lire le sujet avec attention pour comprendre ce qui est demandé.

L'exercice du tableau n'est guère plus compliqué dès l'instant où on a compris l'objectif à atteindre et qu'on a effectué correctement les opérations liminaires. Or les candidats cherchent des obstacles là où une simple lecture du sujet et des documents donnés en annexe permet de faire les choix demandés. On remarque aussi que les candidats ont parfois des difficultés à faire un tableau clair, bien présenté.

La moyenne de l'épreuve a été de 13,12 pour les admissibles externes, et de 10,44 pour les admissibles internes.

- L'épreuve du questionnaire comportait 10 questions faisant appel à des connaissances professionnelles. Ont été posées des questions à choix multiple (QCM) et des questions traditionnelles à réponse rapide ou à réponse rédigée dans la proportion de $4 \mathrm{QCM}, 5$ questions à réponse rapide et 1 question à réponse rédigée (en moins de 10 lignes, maximum recommandé).

On a constaté que trop de candidats sont mal à l'aise dans la rédaction, même lorsqu'ils témoignent de connaissances réelles : maladresses dans l'expression, dans le choix des mots, fautes d'orthographe grossières.

La moyenne de l'épreuve a été de 15,73 pour les admissibles externes (15,25 en 2002), et de $14,59$ pour les admissibles internes (14,24 en 2002$)$.

\section{2) Épreuves orales}

- L'épreuve de classement de fiches, de l'avis unanime du jury, est considérée comme un reliquat sympathique des activités anciennes des bibliothèques. Peu pertinente pour détecter les compétences des candidats, d'un intérêt limité pour la vie quotidienne des magasiniers en chef, elle mériterait d'être remplacée par une épreuve plus proche de la réalité des bibliothèques et plus discriminante.

- L'entretien avec le jury est une épreuve importante pour les candidats, non seulement par son coefficient élevé (coeff. 4), mais aussi et surtout par le contact avec le jury, les émotions à maîtriser, la nécessité de faire un exposé concis en peu de temps, et de répondre aux questions du jury.

On s'aperçoit que peu de candidats ont pris la peine de s'entraîner psychologiquement à cette épreuve, et de préparer concrètement la première partie de leur intervention en la structurant pour ne pas être trop perturbés en cas de défaillance. Pour beaucoup, l'improvisation est de mise et va souvent de pair avec la non maîtrise du temps.

D'une manière générale, les candidats âgés de plus de 45 ans sont mieux préparés, souvent très motivés et incisifs, réagissant vite aux questions posées ; sans doute l'expérience et la maturité jouent-elles un rôle dans leur maîtrise de l'épreuve. En revanche, les candidats plus jeunes (20-30 ans), souvent diplômés du supérieur chez les externes, ont moins de motivation et paraissent plus ternes alors qu'on serait en droit d'attendre une aisance plus grande.

La moyenne de la note d'entretien des admissibles a été de 14,94 au concours externe $(15,95$ en 2002$)$ et de 15,77 au concours interne $(16,77$ en 2002$)$.

En conclusion, on constate que le nombre de candidats inscrits au concours externe s'est stabilisé en 2003 mais qu'il y a davantage de candidats présents aux épreuves écrites notamment. L'augmentation du nombre de candidats inscrits au concours interne se confirme en $2003:+13,2 \%$, comme se confirme l'augmentation du nombre de présents $(+11,4 \%)$. 
Parallèlement, on a constaté une forte augmentation du nombre des notes éliminatoires en particulier au concours interne. Il est naturellement délicat d'interpréter des chiffres sur une courte durée ; il conviendra donc de surveiller l'évolution de ces phénomènes.

\section{Examen professionnel pour l'accès au grade d'assistant des bibliothèques de classe exceptionnelle}

L'ouverture d'un examen professionnel de sélection pour l'accès au grade d'assistant de bibliothèque de classe exceptionnelle au titre de l'année 2003 a été autorisée par un arrêté du 4 août 2003 paru au Journal officiel du 14 août. Ouvertes à partir du $1^{\text {er }}$ septembre 2003, les inscriptions ont été closes le 3 octobre 2002. L'arrêté du 11 septembre 2003 (Journal officiel du 23 septembre) fixait le nombre d'emplois offerts à 27.

Le jury, placé sous la présidence de F. Lemelle, était composé de cinq membres dont un assistant des bibliothèques de classe exceptionnelle. L'examen s'est déroulé du 17 au 21 novembre dans les bureaux du ministère de l'Éducation nationale, rue Dutot.

L'épreuve se déroule de la façon suivante :

Le jury dispose avant l'entretien avec le candidat de son dossier de candidature ; ce dernier comporte, outre une notice, sorte de "fiche d'identité professionnelle", ses différentes "affectations et attributions en qualité d'assistant de bibliothèque". Les dossiers étaient dans l'ensemble correctement remplis.

L'épreuve orale comportait deux phases : un exposé du candidat sur ses fonctions et les tâches qu'il effectue, suivi d'un entretien avec le jury qui permet de développer certains points et d'avoir une interactivité propice pour apprécier la personnalité et les aptitudes du candidat.

L'exposé devait durer 5 minutes et était le point de départ de l'épreuve. Un bon exposé doit retracer clairement et synthétiquement le parcours professionnel de l'agent et mettre l'accent sur ses fonctions présentes en essayant de les resituer dans les activités de l'établissement (présentation succincte de l'établissement, positionnement de l'agent dans l'organigramme). Certains candidats ont réussi cet exercice dans le temps imparti. Beaucoup, qui n'avaient pas suffisamment préparé leur exposé, n'ont pas maîtrisé leur temps de parole et se sont exprimé plus longuement. D'autres, moins nombreux, se sont contentés d'une simple énumération de leurs tâches. Il est donc nécessaire que les candidats se préparent et s'exercent à structurer leur exposé qui ne doit pas pour autant être récité ou lu.

L'entretien qui suit est très important. Il permet de préciser la réalité des fonctions exercées et du travail effectué. C'est un exercice libre où la pratique professionnelle, le goût du service public, le désir d'améliorer ses compétences et de progresser sont appréciés. Il permet également de juger de la facilité d'expression du candidat et de sa capacité à improviser ou à répondre à des questions qu'il n'attendait pas nécessairement.

Sur les 198 agents promouvables, 65 s'étaient inscrits ; 64 se sont présentés et 27 ont été retenus. Le seuil d'admission s'établissait à 15,4.

On trouve sur le site WEB de I'IGB la liste, et le texte complet des rapports des jurys de concours.

www.education.gouv.fr/syst/igb (rubrique "Publications") 
ANNEXE 5

\section{TEXTES CONCERNANT L'INSPECTION GENERALE DES BIBLIOTHEQUES}

- État au 31 décembre 2003 -

\section{A - ORGANISATION} l'Inspection

1) Statut du corps des inspecteurs généraux, missions d'inspection générale, doyen de

- Décret $\mathrm{n}^{\circ}$ 69-1265 du 31 décembre 1969 portant statut du personnel scientifique des bibliothèques, titre III, art. 20 et 21 (succédant au décret $n^{\circ}$ 45-2099 du 13 septembre 1945, relatif à l'effectif et au statut des inspecteurs généraux des bibliothèques, et au décret $n^{\circ}$ 52-554 du 16 mai 1952, relatif aux statuts des personnels scientifiques des bibliothèques titre II, art. 20-22).

- Décret $\mathrm{n}^{\circ}$ 92-26 du 9 janvier 1992 portant statut particulier du corps des conservateurs des bibliothèques et du corps des conservateurs généraux des bibliothèques :

- Art. 3, les conservateurs en chef "peuvent se voir confier par le ministre chargé de l'enseignement supérieur des missions d'inspection générale".

- Art. 23, les conservateurs généraux "peuvent être chargés, par arrêté du ministre chargé de l'enseignement supérieur, de missions d'inspection générale".

- Art. 48, à compter de la date de publication du décret, il n'est plus procédé au recrutement d'inspecteur général des bibliothèques.

- Décret $n^{\circ}$ 2001-946 du 11 octobre 2001 modifiant le décret $n^{\circ}$ 92-26 du 9 janvier 1992 portant statut particulier du corps des conservateurs des bibliothèques et du corps des conservateurs généraux des bibliothèques :

- Art. 2, le dernier alinéa de l'article 3 du décret du 9 janvier 1992 est modifié , les conservateurs en chef "peuvent se voir confier des missions d'inspection générale par arrêté du ministre chargé de l'enseignement supérieur, pris après avis du ministre chargé de la culture".

- Art. 5, le second alinéa de l'article 23 du même décret est remplacé par trois alinéas ainsi rédigés :

Les conservateurs généraux "peuvent se voir confier des missions d'inspection générale par arrêté du ministre chargé de l'enseignement supérieur pris après avis du ministre chargé de la culture.

Parmi les conservateurs généraux chargés de mission d'inspection générale, le ministre chargé de l'enseignement supérieur nomme, par arrêté pris après avis du ministre chargé de la culture, un doyen des conservateurs et conservateurs généraux chargés de missions d'inspection générale.

Le doyen dirige, anime et coordonne les activités des agents chargés de missions d'inspection générale. Il centralise les conclusions de leurs travaux."

- Arrêté du 3 juillet 2002, portant nomination de M. J.-L. Gautier-Gentès comme doyen des conservateurs et conservateurs généraux chargés de mission d'inspection générale.

\section{2) Rattachement}

- Décret n 75-1003 du 29 octobre 1975, transfert au secrétariat d'État à la culture d'attributions dans les domaines du livre et de la lecture publique, art. 3 : l'IGB est placée sous l'autorité du secrétaire d'État aux universités et mise à la disposition du secrétaire d'État à la culture, pour les bibliothèques qui relèvent de sa compétence.

- Arrêté du 18 décembre 1975, art. 1 : relèvent directement du secrétaire d'État, l'Inspection générale de l'administration du ministère de l'éducation pour les missions relatives aux attributions du secrétaire d'État 
aux universités et l'Inspection générale des bibliothèques qui est, d'autre part, à la disposition du secrétaire d'État à la culture pour les bibliothèques relevant de sa compétence.

- Décret $n^{\circ}$ 93-798 du 16 avril 1993 relatif aux attributions du ministre de l'Enseignement supérieur et de la Recherche, art. 2 : l'Inspection générale des bibliothèques est placée sous son autorité.

- Décret $\mathrm{n}^{\circ}$ 93-898 du 12 juillet 1993 portant organisation de l'administration centrale du ministère de l'Enseignement supérieur et de la Recherche, art. 1 : l'Inspection générale des bibliothèques est une composante de l'administration centrale.

- Décret $n^{\circ}$ 95-767 du 8 juin 1995 relatif aux attributions du ministre de l'Éducation nationale, de l'Enseignement supérieur, de la Recherche et de l'Insertion professionnelle :

- Art. 1 : le ministre exerce les attributions respectivement dévolues au ministre de l'Éducation nationale et au ministre de l'Enseignement supérieur et de la Recherche par les décrets du 16 avril 1993.

- Art. 2 : pour l'exercice de ses attributions, dans les domaines de l'éducation, de l'enseignement supérieur et de la recherche, le ministre a autorité sur les services placés sous l'autorité du ministre de l'Éducation nationale et du ministre de l'Enseignement supérieur et de la Recherche par les décrets du 16 avril 1993.

- Décret $\mathrm{n}^{\circ}$ 95-791 du 19 juin 1995 relatif aux attributions du secrétaire d'État à l'enseignement supérieur, art. 2 : pour l'exercice de ses attributions et sous l'autorité du ministre, le secrétaire d'État fait appel, en tant que de besoin, aux directions mentionnées par le décret du 12 juillet 1993 ainsi qu'à l'Inspection générale de l'éducation nationale, l'Inspection générale de l'administration de l'éducation nationale et l’Inspection générale des bibliothèques.

- Décret $n^{\circ}$ 95-1210 du 15 novembre 1995 relatif aux attributions du ministre de l'Éducation nationale, de l'Enseignement supérieur et de la Recherche, art. 1 : le ministre de l'Éducation nationale, de l'Enseignement supérieur et de la Recherche exerce les attributions et les pouvoirs précédemment dévolus au ministre de l'Éducation nationale, de l'Enseignement supérieur, de la Recherche et de l'Insertion professionnelle par le décret du 8 juin 1995 susvisé.

- Décret $n^{\circ}$ 96-16 du 10 janvier 1996 portant organisation de l'administration centrale du ministère de l'éducation nationale, de l'enseignement supérieur et de la recherche, art. 1 : liste des directions composant l'administration centrale "outre les inspections générales, le bureau du cabinet et les hauts fonctionnaires de défense, qui sont directement rattachés au ministre".

- Décret $n^{\circ}$ 97-707 du 11 juin 1997 relatif aux attributions du ministre de l'Éducation nationale, de la Recherche et de la Technologie, art. 3 : pour l'exercice de ses attributions, le ministre de l'Éducation nationale, de la Recherche et de la Technologie a autorité sur les directions, services, délégations et missions énumérées par le décret du 10 janvier 1996 susvisé.

- Décret $\mathrm{n}^{\circ}$ 97-1149 du 15 décembre 1997 portant organisation de l'administration centrale du ministère de l'Éducation nationale, de la Recherche et de la Technologie, art. 1 : liste des directions et délégations composant l'administration centrale, "outre les inspections générales, le bureau du Cabinet et les hauts fonctionnaires de défense, directement rattachés au ministre".

- Décret $\mathrm{n}^{\circ}$ 2001-852 du 18 septembre 2001 modifiant le décret $\mathrm{n}^{\circ}$ 97-1149 du 15 décembre 1997 et portant organisation de l'administration centrale du ministère de l'Éducation nationale et de l'administration centrale du ministère de la Recherche, art. 4 : reprise de l'article $1^{\text {er }}$ du décret du 15 décembre 1997, avec modification de la liste des directions et délégations.

- Décret $\mathrm{n}^{\circ}$ 2002-910 du 29 mai 2002 relatif aux attributions déléguées au ministre délégué à l'Enseignement supérieur et à la Recherche, art. 2 : pour l'exercice de ses attributions, le ministre délégué dispose des directions de... ; en tant que de besoin, des directions de..., de l'Inspection générale de l'éducation nationale, de l'Inspection générale de l'administration de l'éducation nationale et de la recherche, de l'Inspection générale des bibliothèques...

- Décret $\mathrm{n}^{\circ}$ 2002-959 du 4 juillet 2002 relatif aux attributions du ministre de la Jeunesse, de l'Éducation nationale et de la Recherche, art. 4 : pour l'exercice de ses attributions, le ministre a autorité sur les directions..., l'Inspection générale de l'éducation nationale, l'Inspection générale de l'administration de l'éducation nationale et de la recherche, l'Inspection générale des bibliothèques...

- Décret $n^{\circ}$ 2002-981 du 12 juillet 2002 relatif aux attributions déléguées à la ministre déléguée à la Recherche et aux Nouvelles technologies, art. 2 : pour l'exercice de ses attributions, la ministre déléguée 
dispose des directions de... ; en tant que de besoin, des directions de..., de l'Inspection générale de l'éducation nationale, de l'Inspection générale de l'administration de l'éducation nationale et de la recherche, de l'Inspection générale des bibliothèques...

\section{B - INSPECTION, CONTRÔLE. Champ d'inspection, organisation des inspections, coopération}

- Décret $n^{\circ}$ 85-694 du 4 juillet 1985 modifié relatif aux services de la documentation des établissements d'enseignement supérieur, art. 5 et 14 : les services communs de documentation et les services interétablissements de coopération documentaire sont soumis au contrôle de l'Inspection générale des bibliothèques. Celle-ci remplit à leur égard un rôle d'évaluation et de conseil.

- Décret $n^{\circ}$ 91-321 du 27 mars 1991 relatif à l'organisation des services de la documentation des établissements d'enseignement supérieur des académies de Paris, Créteil et Versailles, art. 18: les services interétablissements de coopération documentaire sont soumis au contrôle de l'Inspection générale des bibliothèques, qui remplit à leur égard un rôle d'évaluation et de conseil.

- Lettre de mission du $1^{\text {er }}$ février 1990 du ministre de l'éducation nationale : extension du champ d'action de l'IGB aux grandes écoles, instituts, organes de coopération et patrimoine, l'inspection des grands établissements perd son caractère exceptionnel ; inspections individuelles concernant le personnel menées à la demande du ministre seulement; programme d'études thématiques ; coopération souhaitée avec l'Inspection générale de l'administration de l'éducation nationale (l'arrêté du 15 mars 1984 portant organisation de l'Inspection générale de l'administration de l'éducation nationale prévoit dans son art. 6 le principe de missions communes avec d'autres inspections générales dont celle des bibliothèques).

- Décret n 88-1037 du 9 novembre 1988 relatif au contrôle technique de l'État sur les bibliothèques des collectivités territoriales, pris en application de l'article 61 de la loi n 83-663 du 22 juillet 1983 :

- Art. 6, définition du contrôle technique, qui porte sur les conditions de constitution, de gestion, de traitement, de conservation et de communication des collections et des ressources documentaires et d'organisation des locaux (art. R.341-6 du code des communes),

(Na : l'article 65 de la loi du 22 juillet 1983 prévoit en outre que l'État exerce un contrôle technique sur l'activité du personnel scientifique et technique des communes, départements et régions, chargé de procéder à l'étude, la conservation et la mise en valeur du patrimoine.)

- Art. 7, le contrôle technique de l'État sur les bibliothèques des communes est exercé de façon permanente sous l'autorité du ministre chargé de la Culture par l'Inspection générale des bibliothèques. Le ministre peut également confier des missions spécialisées à des membres du personnel scientifique des bibliothèques ainsi qu'à des fonctionnaires de son ministère choisis en raison de leur compétence scientifique et technique. Le contrôle s'exerce sur pièces et sur place. Chaque inspection donne lieu à un rapport au ministre chargé de la Culture, qui est transmis par le préfet au maire (art. R.341-7 du code des communes).

* Art. 11, les mêmes dispositions sont applicables aux départements et aux régions.

- Arrêté n 89-603 du 2 mars 1989 (ministère de la Culture, ministère de l'Intérieur, secrétariat d'État chargé des collectivités territoriales) : circulaire d'application du décret relatif au contrôle technique de l'État sur les bibliothèques des collectivités territoriales. Ce texte porte sur le champ d'application du contrôle (qui concerne, par exemple, la qualification technique des personnels, au titre de la qualité technique des bibliothèques), les agents chargés de l'exécution du contrôle et les modalités d'exercice de ce contrôle.

- Le Code général des collectivités territoriales a intégré les dispositions relatives au contrôle de l'État sur les bibliothèques des collectivités territoriales :

- Partie législative, articles L.1422-1 et L.1422-8 (codifie les dispositions de la loi du 22 juillet 1983, article 61, $1^{\mathrm{er}}$ alinéa) ; l'article L. 2541-1 précise que ces dispositions sont applicables aux communes des départements de la Moselle, du Bas-Rhin et du Haut-Rhin.

- Partie réglementaire, articles R.1422-3 à R.1422-14 (se substitue à la partie réglementaire du Code des communes, qui codifiait notamment les dispositions du décret du 9 novembre 1988).

- Arrêté du 9 mai 1995 relatif à l'organisation de la direction du Livre et de la Lecture :

- Art. 1, elle exerce le contrôle technique de l'État sur les bibliothèques des collectivités territoriales,

- Art. 7, le programme annuel d'inspection des bibliothèques relevant de la direction est préparé par la direction du Livre et de la Lecture, en relation avec l'Inspection générale des bibliothèques, les 
conservateurs généraux des bibliothèques chargés de missions d'inspection et les directeurs régionaux des affaires culturelles (suivant le décret $n^{\circ}$ 86-538 du 14 mars 1986 relatif aux attributions et à l'organisation des directions régionales des affaires culturelles, art. 2, le DRAC veille à la mise en œuvre du contrôle scientifique, administratif et technique de la réglementation).

Ces articles reprennent les termes des articles 1 et 5 de l'arrêté du 19 mars 1993, relatif à l'organisation de la direction du Livre et de la Lecture. Suivant l'arrêté antérieur du 16 février 1987, sur les missions et l'organisation de la direction du Livre et de la Lecture, art. 7, l'inspection, mise à la disposition du ministre de la Culture, était placée auprès du directeur du Livre et de la Lecture.

Depuis 1993, l'Inspection a reçu des programmes de travail annuels, tant du ministre de l'Éducation nationale que du directeur du Livre et de la Lecture.

\section{C - MISSIONS HORS INSPECTION ET CONTRÔLE}

\section{1) Formation, recrutement, homologation, gestion des personnels}

a) École nationale supérieure des sciences de l'information et des bibliothèques (ENSSIB)

Aucun texte ne prévoit plus la participation de l'Inspection aux instances de l'ENSSIB.

Cependant, un inspecteur général des bibliothèques a assuré l'intérim de la direction de l'École. Un inspecteur général est membre du conseil d'administration de l'ENSSIB.

Des inspecteurs généraux président le conseil de perfectionnement du diplôme de conservateur de bibliothèque, le conseil de perfectionnement de la formation initiale des bibliothécaires, le conseil de perfectionnement de la formation continue.

\section{b) École nationale des chartes}

- Décret $\mathrm{n}^{\circ}$ 87-232 du 8 octobre 1987 relatif à l'École nationale des chartes, art. 12 : un inspecteur général des bibliothèques est membre de droit du conseil scientifique.

Un inspecteur général est en outre membre du conseil d'administration de l'École nationale des chartes depuis l'année 2000.

\section{c) Jurys de recrutement des personnels de catégorie A : conservateurs et bibliothécaires}

Les textes relatifs aux jurys de recrutement des personnels de catégorie A ne prévoient pas de rôle particulier pour les membres de l'Inspection.

Cependant, un inspecteur général préside le jury de recrutement de conservateurs stagiaires réservé aux élèves de l'École des chartes (arrêté annuel de nomination de ce jury). Un inspecteur général est viceprésident du jury de recrutement de conservateurs stagiaires élèves de l'ENSSIB.

En ce qui concerne les bibliothécaires, depuis la constitution de ce corps en 1992, un inspecteur général a constamment présidé les jurys des concours de recrutement de bibliothécaires (externe, interne, interne exceptionnel). Un inspecteur général a également assuré la présidence du jury de l'examen professionnel pour l'accès de non titulaires dans le corps des bibliothécaires, organisé en 2001, et celle du jury du concours réservé de recrutement de bibliothécaires.

d) Bibliothécaires adjoints spécialisés

- Arrêté du 13 avril 2001 fixant les règles d'organisation générale, le programme et la nature des épreuves des concours externe et interne d'accès au corps des bibliothécaires adjoints spécialisés, art. $5:$ le jury comprend un inspecteur général des bibliothèques ou conservateur général des bibliothèques, président. 


\section{e) Assistants des bibliothèques}

- Arrêté du 13 avril 2001 fixant les règles d'organisation générale, le programme et la nature des épreuves des concours externe et interne d'accès au corps des assistants des bibliothèques, art. 5 : le jury comprend un inspecteur général des bibliothèques ou conservateur général des bibliothèques, président.

- Arrêté du 18 avril 2001 fixant les modalités d'organisation de l'examen professionnel pour l'accès au grade d'assistant des bibliothèques de classe exceptionnelle, art. 4 : le jury est composé de quatre membres au moins dont un président, inspecteur général des bibliothèques, conservateur général des bibliothèques ou conservateur en chef des bibliothèques.

\section{f) Personnels de magasinage}

- Arrêté du 6 septembre 1995 fixant les modalités d'organisation des concours de recrutement de magasiniers en chef, art. 4 : le jury comprend un inspecteur général des bibliothèques ou un conservateur général des bibliothèques, président.

- Arrêté du 6 septembre 1995 fixant les modalités d'organisation des concours de recrutement de magasiniers spécialisés, art. 4 : le jury comprend un inspecteur général des bibliothèques ou un conservateur général des bibliothèques, président.

g) Commissions d'homologation chargées d'examiner les demandes d'intégration dans des cadres d'emploi de la fonction publique territoriale

Arrêtés du ministre de l'Intérieur et de l'Aménagement du territoire,

- en date du 27 août 1993, fixant la composition de la commission chargée d'examiner les demandes d'intégration dans le cadre d'emplois des conservateurs territoriaux des bibliothèques,

- en date du 14 décembre 1993, fixant la composition de la commission chargée d'examiner les demandes d'intégration dans le cadre d'emplois des bibliothécaires.

Dans chacune des commissions, un inspecteur général des bibliothèques est membre titulaire et deux IGB sont suppléants. Le statut particulier du cadre d'emplois des conservateurs territoriaux du patrimoine (décret $n^{\circ}$ 91-839 du 2 septembre 1991, art. 38) et le statut particulier du cadre d'emplois des bibliothécaires territoriaux (décret $\mathrm{n}^{\circ}$ 91-845 du 2 septembre 1991, art. 33) prévoient en effet que ces commissions comprennent, parmi leurs membres, trois personnalités dont au moins un fonctionnaire chargé de mission d'inspection.

\section{h) Commissions administratives paritaires des corps de personnels d'État des bibliothèques}

Arrêtés portant désignation des membres des commissions administratives paritaires des personnels des bibliothèques : un inspecteur général est membre titulaire, un inspecteur général est éventuellement suppléant dans chacune des CAP, sauf celle des conservateurs généraux des bibliothèques (à laquelle des inspecteurs généraux assistent à titre d'expert).

\section{2) Suivi des services communs de documentation des universités}

a) Affectation des locaux des bibliothèques universitaires; extension, transfert de sections ou création de nouvelles sections

- Circulaire $\mathrm{n}^{\circ}$ 82-0882 du 25 novembre 1982, adressée aux présidents d'université par le directeur général des Enseignements supérieurs et de la Recherche, circulaire $n^{\circ}$ 82-0900 du 1er décembre 1982, adressée par le directeur des Bibliothèques aux directeurs de bibliothèques universitaires (circulaires préparant le transfert des crédits et des charges d'infrastructure des bibliothèques aux universités, et l'après-transfert) :

- toute initiative visant à attribuer une autre fonction aux locaux des bibliothèques universitaires devra être soumise à l'avis de la direction et de l'inspection générale des bibliothèques,

- pour l'extension, le transfert des sections ou la création de nouvelles sections de bibliothèques universitaires, la direction et l'inspection devront être consultées. 


\section{b) Avis avant nomination des responsables de section}

- Décret $n^{\circ}$ 85-694 modifié du 4 juillet 1985 sur les services de la documentation des établissements d'enseignement supérieur, art. 11 : nomination des responsables de section de bibliothèque par le président de l'université, sur proposition du directeur du service commun de la documentation et après avis de l'Inspection générale des bibliothèques.

\section{c) Retrait des fonctions de direction d'un service commun de la documentation}

- Circulaire $n^{\circ}$ 85-0611 du 10 octobre 1985 : ces fonctions peuvent être retirées par le ministre, soit sur la demande de l'intéressé, soit au vu des rapports de l'Inspection générale des bibliothèques demandés par le ministre, après consultation des présidents ou directeurs d'établissements concernés et après avis de la commission consultative compétente.

\section{3) Participation à diverses instances}

- Arrêté du 2 septembre 1958 fixant les modalités de gestion de la Bibliothèque byzantine, art. 3 : comité consultatif, un IGB membre de droit.

- Arrêté du 9 mai 1989 portant création du conseil scientifique du patrimoine des bibliothèques publiques, art. 3 : un IGB en est membre.

- Arrêté du 27 mars 1991 relatif au comité de la documentation des universités des académies de Paris, Créteil et Versailles, art. 2 : le comité comprend un IGB désigné par le ministre de l'Enseignement supérieur, ou son représentant.

- Décret n 92-45 du 15 janvier 1992 portant organisation de la Bibliothèque nationale et universitaire de Strasbourg et relatif aux services de la documentation des universités de Strasbourg, art. 3 : le conseil d'administration de la BNUS comprend, parmi les membres de droit, un IGB désigné par le ministre de l'Enseignement supérieur.

- Décret $n^{\circ}$ 94-920 du 24 octobre 1994 relatif à la Commission nationale de l'Inventaire général des monuments et des richesses artistiques de la France, art. 1 : la commission comprend un inspecteur général des bibliothèques ou un conservateur de bibliothèque.

- Convention entre le ministère de la Culture et de la Communication, le ministère de l'Éducation nationale, de la Recherche et de la Technologie et le CNRS (Institut de recherche et d'histoire des textes), s'associant pour un programme de recherche sur les manuscrits des bibliothèques municipales et des bibliothèques de l'enseignement supérieur, art. 7 : le comité scientifique de programme comprend un inspecteur général des bibliothèques. La convention validée le 25 mars 2003, a été conclue pour une durée de quatre ans à compter du $1^{\text {er }}$ janvier 2002. 
ANNEXE 6

\section{REPARTITION DES ZONES D'INSPECTION EN 2004}

\begin{tabular}{|c|c|c|c|}
\hline & $\begin{array}{c}\text { RÉGIONS } \\
\text { (Bib. universitaires et territoriales) }\end{array}$ & $\begin{array}{c}\text { ÎLE-DE-FRANCE } \\
\text { (Bibliothèques territoriales) }\end{array}$ & $\begin{array}{l}\text { PARIS-ÎLE-DE-FRANCE } \\
\text { (Bibliothèques universitaires) }\end{array}$ \\
\hline Jean-Marie ARNOULT & $\begin{array}{l}\text { Alsace } \\
\text { Bourgogne } \\
\text { Lorraine }\end{array}$ & Yvelines & $\begin{array}{l}\text { BIU Ste Geneviève } \\
\text { BU St-Quentin-en-Yvelines } \\
\text { Bib. de l'Institut } \\
\text { Bib. Mazarine }\end{array}$ \\
\hline Jean-Luc GAUTIER-GENTÈS & $\begin{array}{l}\text { Aquitaine } \\
\text { Midi-Pyrénées } \\
\text { Pacifique }\end{array}$ & Val-de-Marne & $\begin{array}{l}\text { BIU de la Sorbonne } \\
\text { BIU des Langues orientales } \\
\text { BU Paris III } \\
\text { BU Paris IX } \\
\text { BU Paris XII }\end{array}$ \\
\hline Françoise LEMELLE & $\begin{array}{l}\text { Auvergne } \\
\text { Limousin } \\
\text { Pays-de-la-Loire } \\
\text { Poitou-Charentes }\end{array}$ & Val d'Oise & $\begin{array}{l}\text { BIU Cujas } \\
\text { BU Paris IV } \\
\text { BU de Cergy-Pontoise }\end{array}$ \\
\hline Danielle OPPETIT & $\begin{array}{l}\text { Nord-Pas-de-Calais } \\
\text { Picardie }\end{array}$ & Seine-Saint-Denis & $\begin{array}{l}\text { BIUM } \\
\text { BU Paris VIII } \\
\text { BU Paris XIII } \\
\text { BU Marne-la-Vallée } \\
\text { Bib. de l'Académie de } \\
\text { Médecine }\end{array}$ \\
\hline Denis PALLIER & $\begin{array}{l}\text { Rhône-Alpes } \\
\text { Guadeloupe } \\
\text { Guyane } \\
\text { Martinique }\end{array}$ & 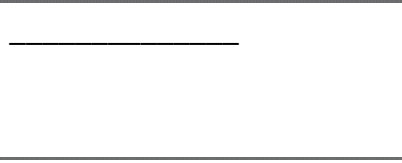 & $\begin{array}{l}\text { BIU Jussieu } \\
\text { BU Paris V } \\
\text { BU Paris VI } \\
\text { BU Paris } 7 \\
\end{array}$ \\
\hline
\end{tabular}


Presidences de JuRYs de Concours et d'EXAMENS en 2004 (ETAT)

\begin{tabular}{|l|l|}
\hline & \multicolumn{1}{|c|}{ IG } \\
\hline Conservateurs d'État & Danielle OPPETIT, Vice-présidente \\
\hline Chartistes/ENSSIB & Denis PALLIER, Président \\
\hline Bibliothécaires & Albert POIROT, Président \\
\hline Bibliothécaires (concours réservé) & Albert POIROT, Président \\
\hline Bibliothécaires adjoints spécialisés & Lydia MERIGOT, Présidente \\
\hline Assistants des bibliothèques & Claudine LIEBER, Présidente \\
\hline Assistants des bibliothèques classe exceptionnelle & Françoise LEMELLE, Présidente \\
\hline Magasiniers en chef & Jean-Marie ARNOULT, Président \\
\hline
\end{tabular}




\section{INFORMATIONS PRATIQUES}

\begin{tabular}{|c|c|}
\hline Courrier & $\begin{array}{l}110 \text { rue de Grenelle } \\
75357 \text { PARIS } 07 \text { SP }\end{array}$ \\
\hline Bureaux & 10 rue Auber, Paris $9^{\text {gème }}$ \\
\hline E卂iti & 01.55 .55 .25 .39 \\
\hline 国 & 01.55.55.05.69. \\
\hline 表吾" & igb@education.gouv.fr \\
\hline Web & www.education.gouv.fr/syst/igb \\
\hline
\end{tabular}

\begin{tabular}{|c|c|c|c|c|}
\hline Jean-Marie ARNOULT & 要 & $\begin{array}{l}01.55 .55 .25 .39 \\
01.40 .15 .73 .65\end{array}$ & 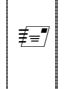 & $\begin{array}{l}\text { igb@education.gouv.fr } \\
\text { jean-marie.arnoult@culture.gouv.fr }\end{array}$ \\
\hline Jean-Luc GAUTIER-GENTÈS（Doyen) & 留 & 01.55 .55 .25 .92 & 至三 & jean-luc.gautier-gentes@education.gouv.fr \\
\hline Delphine LE BIAN & 澛 & 01.55.55.25.39 & 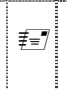 & $\begin{array}{l}\text { delphine.le-bian@education.gouv.fr } \\
\text { igb@education.gouv.fr }\end{array}$ \\
\hline Françoise LEMELLE & 留 & 01.55 .55 .25 .96 & 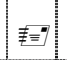 & francoise.lemelle@education.gouv.fr \\
\hline Claudine LIEBER & 留 & $\begin{array}{l}01.55 .55 .25 .39 \\
01.40 .15 .73 .68\end{array}$ & $\pm=$ & $\begin{array}{l}\text { igb@education.gouv.fr } \\
\text { claudine.lieber@culture.gouv.fr }\end{array}$ \\
\hline Lydia MERIGOT & 照 & 01.55 .55 .25 .94 & 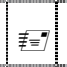 & lydia.merigot@education.gouv.fr \\
\hline Danielle OPPETIT & 留 & 01.55.55.25.95 & 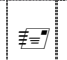 & danielle.oppetit@education.gouv.fr \\
\hline Denis PALLIER & 西 & 01.55.55.25.41. & 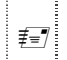 & denis.pallier@education.gouv.fr \\
\hline Albert POIROT & 政 & $\begin{array}{l}01.55 .55 .25 .39 \\
01.40 .15 .75 .36 \\
03.80 .49 .98 .28 \\
\text { Idem }\end{array}$ & 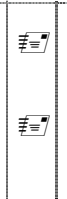 & $\begin{array}{l}\text { igb@education.gouv.fr } \\
\text { albert.poirot@culture.gouv.fr } \\
\text { albert.poirot@culture.gouv.fr }\end{array}$ \\
\hline Daniel RENOULT & 菑 & 01.40.46.24.59. & 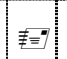 & Daniel.Renoult@rectorat.sorbonne.fr \\
\hline
\end{tabular}

